\title{
Effects of trophic status, water level, and temperature on shallow lake metabolism and metabolic balance: A standardized pan-European mesocosm experiment
}

Ulrike Scharfenberger (10 https://orcid.org/0000-0001-9564-5327, Erik Jeppesen, Meryem Beklioğlu, Martin Søndergaard (1) https://orcid.org/0000-0002-1514-0482, David G. Angeler, Ayşe İdil Çakıroğlu, Stina Drakare (1) https://orcid.org/0000-0002-7389-2105, Josef Hejzlar (10 https://orcid.org/0000-0002-7186-4776, Aldoushy Mahdy, Eva Papastergiadou (i) https://orcid.org/0000-0003-4430-878X, Michal Šorf 는 https://orcid.org/0000-0002-9368-6421, Konstantinos Stefanidis (1) https://orcid.org/0000-0002-9121-3188, Arvo Tuvikene (1) https://orcid.org/0000-0001-55201166, Priit Zingel (i) https://orcid.org/0000-0003-1629-2063, Rita Adrian 는 https://orcid.org/0000-0002-6318-7189

DOI

10.1002/Ino.11064

Original publication date

02 November 2018

Document version

Accepted version

Published in

Limnology and Oceanography

Citation (Vancouver)

Scharfenberger U, Jeppesen E, Beklioğlu M, Søndergaard M, Angeler DG, Çakıroğlu Ai, et al. Effects of trophic status, water level, and temperature on shallow lake metabolism and metabolic balance: A standardized pan-European mesocosm experiment. Limnology and Oceanography. 2019;64(2):616-31. 
1 Title: Effects of trophic status, water level, and temperature on shallow lake metabolism and

2 metabolic balance: A standardized pan-European mesocosm experiment

3

4 Running head: Shallow lakes metabolism

5

6 List of authors: Ulrike Scharfenberger ${ }^{1,2}$, Erik Jeppesen ${ }^{3,4}$, Meryem Beklioğlu ${ }^{5,6}$, Martin

7 Søndergaard ${ }^{3}$, David G. Angeler ${ }^{7}$, Ayşe İdil Çakıroğlu ${ }^{8}$, Stina Drakare ${ }^{7}$, Josef Hejzlar ${ }^{9}$, Aldoushy

8 Mahdy $^{10}$, Eva Papastergiadou ${ }^{11}$,Michal Šrf ${ }^{9,12}$, Konstantinos Stefanidis ${ }^{13}$, Arvo Tuvikene ${ }^{14}$, Priit

9 Zingel $^{14}$, Rita Adrian ${ }^{1,2}$

\section{Institute of origin:}

$12{ }^{1}$ Department of Ecosystem Research, Leibniz Institute of Freshwater Ecology and Inland

13 Fisheries, (IGB), Müggelseedamm 310, 12587 Berlin, Germany

$14{ }^{2}$ Department of Biology, Chemistry, Pharmacy, Freie Universität Berlin, Takustr. 3, 14195

15 Berlin, Germany

$16{ }^{3}$ Department of Bioscience and the Arctic Research Centre, Aarhus University, Silkeborg,

17 Denmark

$18{ }^{4}$ Sino-Danish Centre for Education and Research (SDC), Beijing, China

$19{ }^{5}$ Department of Biology, Limnology Laboratory, Üniversiteler Mahallesi, Middle East Technical

20 University, Dumlupınar Bulvarı, 06800, Çankaya, Ankara, Turkey

$21{ }^{6}$ Kemal Kurdas, Ecological Research and Training Station, Lake Eymir, Middle East

22 Technical University, Oran Mahallesi, 06400 Çankaya, Ankara, Turkey 
$23{ }^{7}$ Department of Aquatic Sciences and Assessment, Swedish University of Agricultural Sciences,

24 Uppsala, Sweden

$25{ }^{8}$ Mechanical Engineering Department, Koc University, Rumelifeneri Yolu, 34450 Sarıyer

26 Istanbul, Turkey

$27{ }^{9}$ Institute of Hydrobiology, Biology Centre of the Czech Academy of Sciences, Na Sádkách 7,

2837005 České Budějovice, Czech Republic

$29{ }^{10}$ Department of Zoology, Faculty of Science, Al-Azhar University (Assiut Branch), Assiut

3071524, Egypt

$31{ }^{11}$ Department of Biology, University of Patras, University campus, 26504, Rio, Greece.

$32{ }^{12}$ Faculty of Science, University of South Bohemia, Branišovská 31, 37005 České Budějovice,

33 Czech Republic

$34{ }^{13}$ Department of Water Resources and Environmental Engineering, School of Civil Engineering,

35 National Technical University of Athens, 5, Iroon Politechniou Street, Zografou, Athens, Greece

$36{ }^{14}$ Centre for Limnology, Institute of Agricultural and Environmental Sciences, Estonian

37 University of Life Sciences, Tartumaa, 61117 Rannu, Estonia

38

39 List of institutional email address for each author:

40 Ulrike Scharfenberger ${ }^{1,2}$ - scharfenberberger@igb-berlin.de,

41 Erik Jeppesen ${ }^{3,4}-\mathrm{ej} @ \mathrm{dmu} . \mathrm{dk}$

42 Meryem Beklioğlu ${ }^{5,6}$ - meryem@metu.edu.tr

43 Martin Søndergaard ${ }^{3}-\mathrm{ms} @$ dmu.dk

44 David G. Angeler ${ }^{7}$ - david.angeler@slu.se

45 Ayşe İdil Çakıroğlu ${ }^{8}$ - acakiroglu@ku.edu.tr 2 
46 Stina Drakare ${ }^{7}$ - Stina.Drakare@slu.se

47 Josef Hejzlar ${ }^{9}$ - Josef.Hejzlar@seznam.cz

48 Aldoushy Mahdy ${ }^{10}$ - aldoushy@azhar.edu.eg

49 Eva Papastergiadou ${ }^{11}$ - evapap@upatras.gr

50 Michal Šorf ${ }^{9,12}$ - michal.sorf@centrum.cz

51 Konstantinos Stefanidis ${ }^{13}$ - kstefani@chi.civil.ntua.gr

52 Arvo Tuvikene ${ }^{14}$ - arvo.tuvikene@emu.ee

53 Priit Zingel ${ }^{14}$ - priit.zingel@emu.ee

54 Rita Adrian ${ }^{1,2}$ - adrian@igb-berlin.de

55

56 Corresponding author: Ulrike Scharfenberger, Telephone: +49 3064 181 690, Fax: +49 3064

57181 663, email: scharfenberger@igb-berlin.de

58

59 Keywords: Metabolism, shallow lakes, trophic state, water level change, temperature threshold,

60 climate change 


\section{Abstract}

63 Important drivers of gross primary production (GPP) and ecosystem respiration (ER) in lakes are

64 temperature, nutrients, and light availability, which are predicted to be affected by climate

65 change. Little is known about how these three factors jointly influence shallow lakes metabolism

66 and metabolic status as net heterotrophic or autotrophic. We conducted a pan-European

67 standardized mesocosm experiment covering a temperature gradient from Sweden to Greece to

68 test the differential temperature sensitivity of GPP and ER at two nutrient levels (mesotrophic or

69 eutrophic) crossed with two water levels (1 and $2 \mathrm{~m})$ to simulate different light regimes. The

70 findings from our experiment were compared with predictions made according the metabolic

71 theory of ecology (MTE). GPP and ER were significantly higher in eutrophic mesocosms than in

72 mesotrophic ones, and in shallow mesocosms compared to deep ones, while nutrient status and

73 depth did not interact. The estimated temperature gains for ER of $\sim 0.62 \mathrm{eV}$ were comparable with

74 those predicted by MTE. Temperature sensitivity for GPP was slightly higher than expected

$75 \sim 0.54 \mathrm{eV}$, but when corrected for daylight length, it was more consistent with predictions from

76 MTE $\sim 0.31 \mathrm{eV}$. The threshold temperature for the switch from autotrophy to heterotrophy was

77 lower under mesotrophic $\left(\sim 11^{\circ} \mathrm{C}\right)$ than eutrophic conditions $\left(\sim 20^{\circ} \mathrm{C}\right)$. Therefore, despite a lack

78 of significant temperature-treatment interactions in driving metabolism, the mesocosm's nutrient

79 level proved to be crucial for how much warming a system can tolerate before it switches from

80 net autotrophy to net heterotrophy.

81

82 
Introduction

84 The balance between gross primary production (GPP) and ecosystem respiration (ER) determines

85 the metabolic status of lakes and has a decisive influence on their role in regional/global matter

86 and energy cycles (Andersson \& Sobek, 2006; Brothers et al., 2013; Pacheco et al., 2013).

87 Shallow lakes are the most numerous lake type on Earth (Cael et al., 2017), and have been

88 recognized as hotspots of carbon turnover (Cole et al., 2007; Tranvik et al., 2009). While

89 oligotrophic lakes with high allochthonous carbon inputs tend to be predominantly net

90 heterotrophic $(\mathrm{GPP}<\mathrm{ER})$, many eutrophied lakes have been found to be net autotrophic (GPP >

91 ER) (del Giorgio \& Peter, 1994; Cole et al., 2000; Balmer \& Downing 2011). Lakes can switch

92 between net autotrophy and net heterotrophy across multiple timescales (i.e. daily, weekly, or

93 seasonal) (Staehr \& Sand-Jensen 2007; Coloso et al., 2011; Sadro et al., 2011; Laas et al., 2012);

94 in temperate lakes, the extent of net autotrophy in spring and summer can be a determining factor

95 for the annual metabolic status of lakes (Staehr et al., 2010; Laas et al., 2012). Autotrophic and

96 heterotrophic metabolic pathways are susceptible to changes in light regime, nutrient status, and

97 temperature. All these drivers are predicted to be affected by climate change due to alterations in

98 water levels, nutrient cycling and run-off from the catchment (Coops et al., 2003; Nickus et al.,

99 2010; Jeppesen et al., 2015).

100

101 The metabolic theory of ecology (MTE) provides a comprehensive theoretical framework to

102 investigate metabolic rates' dependence on temperature (Brown et al., 2004). Based on first

103 principles the MTE allows the scaling of metabolic rates from individual biochemical reactions

104 up to the level of ecosystems (Enquist et al., 2003; Allen et al., 2005; Yvon-Durocher et al.,

105 2010b). Independent of temperature, the absolute metabolic rate at the ecosystem level is 5 
primarily determined by the size and abundance distribution of the constituting community of the ecosystem. On the other hand, the physiological dependence of metabolic rates on temperature, approximated by the Arrhenius equation, is still governed by the rate-limiting biochemical process of the cellular level, even at the ecosystem level (Bernacchi et al., 2001; Gillooly et al., 2001; Allen et al., 2005). Under non-limiting conditions, the MTE assumes activation energies of

$111 \approx 0.3 \mathrm{eV}$ (photosynthesis) and $\approx 0.6 \mathrm{eV}$ (respiration). Therefore, in a warming world the MTE

112 predicts a shift towards heterotrophy (as temperatures increase) or even a switch from net

113 autotrophy to net heterotrophy if stored or allochthonous carbon sources are available (Yvon-

114 Durocher et al., 2010a; Laas et al., 2012; Weyhenmeyer et al., 2015). A shift towards

115 heterotrophy would imply a reduction in the carbon sequestration capacity or even loss of this

116 important ecosystem service as a carbon sink, unless offset by sedimentation rates. The

117 temperature at which a net autotrophic system switches to net heterotrophy depends, however,

118 not only on the differential temperature sensitivity of GPP and ER, but also on the ratio of the

119 absolute GPP and ER rates. Theoretically, the more GPP exceeds ER at a given reference

120 temperature, the more warming a lake can tolerate before switching from net autotrophy to net 121 heterotrophy.

123 Since temperature and eutrophication are regarded as the two major stressors for lake ecosystems,

124 several studies have documented their effects on GPP, ER, and the balance between them.

125 Several of these studies have confirmed the occurrence of positive effects of temperature on both

126 ER and GPP, but negative effects on net ecosystem production (NEP = GPP - ER) (Kosten et al.,

127 2010; Moss 2010, Yvon-Durocher et al., 2010a,b, 2012). Moreover, there is general agreement

128 that elevated nutrient concentrations promote metabolic rates, but have greater impact on GPP 6 
129 than ER, causing increases in NEP, or decreases in ER/GPP ratios (del Giorgio \& Peters, 1994;

130 Hanson et al., 2003; Duarte et al, 2004; Staehr et al., 2010) as well as a stronger coupling

131 between ER and GPP in oligotrophic than in eutrophic lakes (Solomon et al., 2013). However,

132 the interacting effects of temperature and trophic state on ecosystem metabolism within the

133 context of already observed and predicted changes in lake water levels, and thus light conditions,

134 are not well understood (Anderson-Teixeira \& Vitousek, 2012; Cross et al., 2015; Welter et al.,

135 2015), and results from experiments on nutrient-temperature interactions are ambiguous

136 (Berggren et al., 2010, Moss 2010, Liboriussen et al., 2011).

137

138 In particular, it is unclear how interactions between temperature, nutrients, and light availability

139 can modify the MTE predicted values for the activation energy resulting in deviations of the

140 apparent temperature sensitivity at ecosystem level from the physiological one (Cross et al.,

141 2015), thereby modifying the MTE-predicted shift towards heterotrophy with increasing

142 temperature. Models combining Arrhenius and Michaelis-Menten kinetics have shown, for

143 instance, that substrate limitation and trophic structure can dampen the apparent temperature

144 sensitivity (Davidson et al., 2012; Davidson et al., 2015). In addition, both the maximum rate and

145 the half-saturation constant were found to increase with increasing temperature in photosynthesis-

146 irradiance relations (Kirk, 2010). However, temperature-dependent increases in the

147 photosynthetic rate might be subdued if phosphorus limits the process (Wykoff et al., 1998; Kirk,

148 2010). In accordance with this, Staehr \& Sand-Jensen (2006) found a reduced metabolic response

149 in a natural algae assembly to increased temperatures under nutrient-limiting conditions. Reduced

150 light and nutrient conditions may affect ER either due to substrate limitation or changes in food

151 quality (McFetters \& Frost, 2011). However, results from laboratory experiments are difficult to 7 
152 scale to the ecosystem level, where covariation of temperature with factors such as water level,

153 daylight length, nutrient cycling, and $\mathrm{N}_{2}$ fixation, as well as acclimation and adaptation at species

154 or community levels can all influence the apparent temperature sensitivity (Atkin \& Tjoelker,

155 2003; Anderson-Teixeira \& Vitousek, 2012; Welter et al., 2015).

156

157 Based on a standardized pan-European mesocosm experiment, this study aims to improve the

158 understanding of how the combined effects of water temperature, water level, and nutrient status

159 affect metabolic rates in shallow lake ecosystems. Specifically, we investigated temperature

160 effects on GPP, ER, and the ratio of ER/GPP under eutrophic and mesotrophic nutrient

161 conditions and at two depth levels, simulating different light conditions. The findings from our

162 experiment were compared with predictions made according to the theoretical framework of the

163 MTE. We tested the following hypotheses: (1) Metabolic rates are lower with reduced nutrient

164 availability, but increase at lower water levels due to higher light availability; (2) a shift towards

165 heterotrophy occurs with increasing temperature due to a higher physiological temperature

166 sensitivity of ER compared with GPP; (3) the apparent temperature sensitivity of ER and GPP

167 will differ between treatments due to interactions between temperature, the availability of light

168 and nutrients; (4) switching between auto- and heterotrophy occur at lower temperatures if the

169 magnitude of ER and GPP is more similar (NEP near zero). We expect this situation under low-

170 nutrient and low-light conditions that sustain a generally lower lake GPP. 


\section{Material and Methods}

172 Experimental design and sampling - The mesocosm experiment was conducted in six European 173 countries, encompassing a climate gradient from Sweden to Greece (Table 1) from May until 174 November 2011. The fiberglass mesocosms used were produced by the same manufacturer, and 175 had a diameter of $1.2 \mathrm{~m}$ and heights of 1.2 or $2.2 \mathrm{~m}$. The mesocosms were set up within the lakes 176 to ensure a natural and ambient water temperature regime, but were otherwise isolated from the 177 surrounding water. In each country, the experiment involved a 2 x 2 factorial design with four 178 replicates; measurements were taken monthly. The first factor involved two different water 179 levels: $1 \mathrm{~m}$ (shallow $-\mathrm{S})$ and $2 \mathrm{~m}($ deep - D) deep mesocosms. These two depths coincided with 180 different mixing depths, since the water in the mesocosms was constantly circulated from bottom 181 to top by standard aquarium pumps, entailing different light conditions (S3 Figure 1). Water 182 levels were allowed to fluctuate with precipitation and evaporation. The second factor involved 183 nutrient manipulation to simulate mesotrophic (low - L) and eutrophic (high $-\mathrm{H})$ conditions.

184 Nutrients were adjusted to the two conditions by monthly nutrient addition aiming at initial 185 concentrations after loading of $25 \mu \mathrm{g}$ phosphate $(\mathrm{P}) \mathrm{L}^{-1}\left(\mathrm{Na}_{2} \mathrm{HPO}_{4}\right)$ and $0.5 \mathrm{mg}$ nitrogen $(\mathrm{N}) \mathrm{L}^{-1}$ $186\left(\mathrm{Ca}\left(\mathrm{NO}_{3}\right)_{2}\right)$ in the mesotrophic and $200 \mu \mathrm{g} \mathrm{P} \mathrm{L}^{-1}$ and $2 \mathrm{mg} \mathrm{N} \mathrm{L}^{-1}$ in the eutrophic treatment. The 187 experiment was synchronized using a common protocol to facilitate comparability (Landkildehus 188 et al., 2014).

190 The mesocosms contained a $10 \mathrm{~cm}$ sediment layer of $90 \%$ washed sand and $10 \%$ natural

191 sediment from an oligotrophic lake, situated near the respective experimental site. To prevent

192 prolonged internal P loading (low nutrient conditions) or P retention (high nutrient conditions) at 193 the start of the experiment, the sediment was acclimatized to the desired phosphate 9 
concentrations for at least two months in the laboratory beforehand. Filtered (500 $\mu \mathrm{m}$ mesh) lake

195 water was used in the mesocosms in all countries except Germany and the Czech Republic,

196 where tap water was used because the P level exceeded the $25 \mu \mathrm{g} \mathrm{TP} \mathrm{L}{ }^{-1}$ threshold of the low-

197 nutrient treatment. The initial $\mathrm{P}$ and $\mathrm{N}$ loadings were adjusted in all high-nutrient mesocosms to

198 obtain the desired nutrient concentration.

199

200 The ability of natural flora and fauna to adapt to the specific climate and nutrient conditions was

201 ensured by using an inoculum of plankton and sediment collected from five different local lakes,

202 covering a nutrient gradient from 25 to $200 \mu \mathrm{g} \mathrm{TP} \mathrm{L}^{-1}$ in each country. Macrophytes

203 (Myriophyllum spicatum) and planktivorous fish were added to all mesocosms. Monthly samples

204 were analyzed for water chemistry and chlorophyll $a(\mathrm{Chl} a)$ in laboratories and on site by using

205 comparable, standard methods (see S2 Table 1). Concomitantly, macrophyte biomass was

206 quantified as plant volume inhabited (PVI [\%]). After each sampling event, 24-hour measurement

207 of dissolved oxygen and water temperature was conducted at two-hour intervals using a multi-

208 parameter probe (for sampling dates, see S1 Table 1). In addition, light profiles of the water

209 column were measured at midday at $10 \mathrm{~cm}$ intervals. For details on the design and sampling, see

210 Landkildehus et al. (2014).

211

212 Data preparation - The study utilized the data collected between July and November, under the

213 assumption that all systems would have had enough time to adjust to the experimental

214 manipulation by then. Seven mesocosms were excluded from the analysis (2 DH, 1 DL and $1 \mathrm{SH}$

215 mesocosm in Germany and $2 \mathrm{SH}$ and $1 \mathrm{SL}$ mesocosm in the Czech Republic) as they were lost

216 during storm events. The analysis is thus based on five measurements, including data from 89 10 
mesocosms per measurement occasion. In Greece, massive water loss due to evaporation

218 prevented sampling in the shallow mesocosms, involving light profiles (from September

219 onwards), water chemistry (from October onwards) and the 24-hour measurement in November.

220 Since visual inspection of these shallow mesocosms indicated high light attenuation, we assumed

221 the same high attenuation for the remaining sampling occasions. Light profiles were also missing

222 for August and September for the Estonian mesocosms. Here, the missing attenuation coefficients

223 were linearly interpolated since none of the attenuation coefficients from the other countries

224 indicated strong seasonality.

225 All data were visually inspected at the raw data level and outliers were identified using boxplots.

226 Only extreme outliers (larger than 3 times the interquartile range) were removed from the data

$227\left(\mathrm{O}_{2}: 36\right.$ values $(1 \%)$ and water temperature: 4 values $\left.(0.1 \%)\right)$ and replaced by interpolated values.

228 Single gaps in the 24-hour data were substituted by values from a polynomial model of degree 4

229 of time; for all other data linear interpolation were used. Reported average values (e.g. average

230 air temperature) correspond to the sampling periods listed in S1 Table 1.

231

232 Estimation of reaeration coefficient $\left(K_{a, 20}\left[h^{-1}\right]\right)$ at $20^{\circ} \mathrm{C}$ for $\mathrm{O}_{2}$ - To ensure minimal influence

233 from respiration, gas exchange was measured when water temperatures were low and after the

234 last sampling in late October, or early November. Under continuous mixing, oxygen saturation

235 was lowered to approx. $30 \%$ by bubbling $\mathrm{N}_{2}$ into two randomly chosen shallow and deep

236 mesocosms. At nightfall, oxygen reduction was completed and oxygen recovery was monitored

237 overnight (reaeration). For each mesocosm, a transport coefficient $\mathrm{K}_{\mathrm{L}, 20}$ was estimated following

238 Liboriussen et al. (2011). Two different respiration models were tested: $\mathrm{R}_{20^{\circ} \mathrm{C}} \cdot 1.047^{(\mathrm{T}-20)}$

239 (Erlandsen \& Thyseen, 1983) and $\mathrm{R}_{20^{\circ} \mathrm{C}} \cdot 1.07^{(\mathrm{T}-20)}$ (Streeter \& Phelps, 1925; De Matos et al., 11 
240 2014). The model outputs did not differ systematically and differences were generally low. Since

241 both models are plausible representations of the temperature effect on respiration, we synthesized

242 the results into an average $\mathrm{K}_{\mathrm{L}, 20}$ value $\left(0.0218 \mathrm{~m} \mathrm{~h}^{-1}\right)$. However, values from the Czech

243 mesocosms were excluded because the $\mathrm{O}_{2}$ reductions were too low to permit proper calculations.

244 Averaging was chosen to appropriately reflect modeling uncertainty, which is in line with the

245 idea of ensemble modeling. $\mathrm{K}_{\mathrm{a}, 20}$ values were then derived by dividing by the mixing depth.

247 Estimation of GPP and ER - Metabolic rates were estimated based on the 24-hour $\mathrm{O}_{2}$

248 measurements using the free-water method following Jeppesen et al. (2012). Since the main focus

249 of the investigation was to analyze the temperature response of the metabolic rate, Arrhenius-type

250 corrections based on a priori Q10 values from the literature were avoided.

251

252 To assess the uncertainty of the estimated metabolic rates, we used a bootstrap approach similar

253 to the one described in Solomon et al. (2013). Estimates with standard errors larger than the

254 estimate itself, and estimates explaining $<5 \%$ of the variability of the 24-hour dissolved oxygen

255 curve, were excluded from further analysis, totaling 14\% of the values. Overall, 374 data points

256 remained. For an overview of the distribution of data points per country, month, and treatment,

257 see Table 2 in S1. To obtain daylight length-corrected GPP values, $\operatorname{GPP}_{\mathrm{dl}}\left[\mathrm{mg} \mathrm{m}^{-3} \mathrm{hd}^{-1}\right]$, GPP per

258 day was divided by the average daylight period, LP $\left(\mathrm{hd}^{-1}\right)$, according to month and country. For

259 further details on the estimation of metabolic rates and the meteorological data used, see

260 Supplement S2. 
Estimation of light attenuation coefficient, $K_{d}$, mean available light and effective light period-

263 For each light profile, an attenuation coefficient, $\mathrm{K}_{\mathrm{d}}$, was estimated based on the Beer-Lambert

264 law. Mean available light over the water column (MAL) was estimated following Staehr et al.

265 (2010). The effective light period ( $\mathrm{LP}_{\text {eff }}$ ), describing the effective light period due to mixing, was

266 calculated following Shatwell et al. (2012). For more details, see Supplement S2.

267

268

Hypotheses generation based on MTE - We used the framework of the MTE to formally derive

the expected temperature dependence of the measured metabolic rates and the ratio between them, which we tested against our experimental findings. Following the MTE, the temperature dependence of metabolic rates can be approximated by the Arrhenius equation within a temperature range of 0 to $30^{\circ} \mathrm{C}$ (Gillooly et al., 2001; Brown et al., 2004; Allen et al., 2005). At

273 the ecosystem level, the MTE is formulated as:

$$
M(T)=M_{0} \exp \left(\frac{-E}{k T}\right)
$$

274 where $\mathrm{M}(\mathrm{T})$ is the temperature-dependent metabolic rate; $\mathrm{M}_{0}$ at the ecosystem level can be

275 interpreted as the size-dependent basic metabolic flux summed over all autotrophs or

276 heterotrophs, respectively, per unit volume (Allen et al., 2005); E is the activation energy and

277 expresses the strength of the temperature effect on the metabolic rate; $\mathrm{k}$ is the Bolzmann constant

$278\left(8.62 \times 10^{-5} \mathrm{eVK}^{-1}\right)$; and $\mathrm{T}$ is the absolute temperature in Kelvin. The above temperature effect

279 can be conveniently analyzed and plotted with Arrhenius plots based on the logarithmized

280 version of equation (1). In Arrhenius plots, the natural logarithm of the metabolic rate is plotted

281 against the inverse and scaled temperature 1/(kT) so that the slope of this linear relationship

282 represents the activation energy and the intercept the absolute metabolic rate of a particular 
metabolic process. The absolute metabolic rate is usually shifted to a biological meaningful

284 reference temperature (Tc), here to $15^{\circ} \mathrm{C}$, following Yvon-Durocher et al. (2010b), and Demars

285 et al. (2011). Thus, the MTE equation used to analyze the temperature dependence of metabolic

286 rates reads:

$$
\ln M(T)=\ln M_{T c}+E \frac{1}{k}\left(\frac{1}{T c}-\frac{1}{T}\right)
$$

287 At the ecosystem level, resource availability could either affect the absolute metabolic rate or the 288 apparent activation energy, E.

290 To derive the expected temperature effects for the ER/GPP ratio, we assumed, following Yvon-

291 Durocher et al. (2010b), that our systems were in a non-steady state and that ecosystem

292 respiration is mainly driven by heterotrophic metabolism, unconstrained by net primary

293 production (for data-driven justification of the assumption, see S6). Thus, the temperature-driven

294 change of the ratio between ER and GPP can be simplified to:

$$
\frac{E R}{G P P}(T)=\frac{E R_{0}}{G P P_{0}} \exp \left(\frac{E_{p}-E_{r}}{k T}\right)
$$

295 where $\mathrm{ER} / \mathrm{GPP}(\mathrm{T})$ is the temperature-dependent metabolic ratio; $\mathrm{ER}_{0}$ and $\mathrm{GPP}_{0}$ are the absolute 296 metabolic rates according to the definition of $\mathrm{M}_{0}$; and $\mathrm{E}_{\mathrm{p}}$ and $\mathrm{E}_{\mathrm{r}}$ are the activation energies for

297 GPP and ER, respectively.

299 Again, the Arrhenius plot together with a shift to a biological meaningful reference temperature 300 can be used to analyze and depict the relationship in logarithmic terms: 


$$
\ln \frac{E R}{G P P}(T)=\ln \frac{E R_{T c}}{G P P_{T c}}-\frac{E_{p}-E_{r}}{k}\left(\frac{1}{T c}-\frac{1}{T}\right)
$$

301 where $\mathrm{GPP}_{\mathrm{Tc}}$ and $\mathrm{ER}_{\mathrm{Tc}}$ are the GPP and ER rate at the reference temperature, Tc; and $\mathrm{E}_{\mathrm{p}}$ and $\mathrm{E}_{\mathrm{r}}$

302 are the activation energies for GPP and ER, respectively. Equations (2) and (4) explicitly state

303 that changes in the metabolic balance with changing temperature, and thus its influence on the

304 carbon sequestration capacity, depend solely on the amount of differential temperature sensitivity

305 between ER and GPP

307 Based on the MTE, we derived an expectation about the temperature at which the switch from

308 autotrophy to heterotrophy occurs. Formally, this is the point of equality between GPP and ER,

309 given by:

$$
T=\frac{1}{\frac{k \ln \frac{G P P_{T c}}{E R_{T c}}}{E_{p}-E_{r}}+\frac{1}{T c}}
$$

310 For fixed $E_{p}$ and $E_{r}$, the switch point depends solely on the ratio between $\mathrm{GPP}_{\mathrm{Tc}}$ and $\mathrm{ER}_{\mathrm{Tc}}$, i.e. the 311 smaller the $\mathrm{ER}_{\mathrm{Tc}}$ relative to $\mathrm{GPP}_{\mathrm{Tc}}$, the higher the temperature at which the system switches from 312 autotrophy to heterotrophy. Thus, assuming resource-dependent absolute $\mathrm{ER}_{\mathrm{Tc}} / \mathrm{GPP}_{\mathrm{TC}}$ values, the 313 switch point from autotrophy to heterotrophy should be lower in nutrient-reduced environments.

315 Statistical analysis - All statistical analyses were conducted using R version 3.1.3 (R Core Team, 316 2015). We analyzed temperature and treatment effects based on monthly data from all countries using linear mixed effect models ("lme4" package, Bates et al., 2014). The following basic model was applied: 
$n M_{c,(s, m), i}^{(D \times N)}(T)=\overline{\ln M_{T c}^{(D \times N)}}+\epsilon_{R}^{c}+\epsilon_{R}^{c, s}+\epsilon_{R}^{c, m}+\left(\overline{E^{(D \times N)}}+\epsilon_{E}^{c}+\epsilon_{E}^{c, m}\right) \frac{1}{k}\left(\frac{1}{T c}-\frac{1}{T}\right)+\epsilon_{c,(s, m), i}$

319

where $\ln M_{c,(s, m), i}^{(D \times N)}$ and $\epsilon_{c,(s, m), i}$ are the temperature-dependent metabolic rate and associated

320

random error for measurement $\mathrm{i}$ of mesocosm $\mathrm{m}$ in month $\mathrm{s}$ and country $\mathrm{c} ; \mathrm{k}$ is the Boltzmann

321

constant; and Tc is the reference temperature set to $15^{\circ} \mathrm{C}$ as in Equations (2) and (4).

$322 \overline{\ln M_{T c}^{(D \times N)}}$ and $\overline{E^{(D \times N)}}$ are the logarithmic average metabolic rate at Tc, and the average apparent

323 activation energy, respectively, for each treatment. For the average metabolic rate at $15{ }^{\circ} \mathrm{C}$,

324 random effects at the level of country $\left(\epsilon_{R}^{c}\right)$, month $\left(\epsilon_{R}^{c, s}\right)$, and mesocosm $\left(\epsilon_{R}^{c, m}\right)$ were considered,

325 where both month and mesocosm are nested within country. For the average apparent activation

326 energy, random effects on the level of country $\left(\epsilon_{E}^{c}\right)$ and mesocosm $\left(\epsilon_{E}^{c, m}\right)$ were taken into account

327 as well. The random effect on the level of mesocosm was nested within country. Following

328 Yvon-Durocher et al. (2012), the random effect of month was added to control for confounding

329 effects on apparent activation energy, which a potential covariation between monthly absolute

330 metabolic rates and temperature may cause. With this modeling approach, we assumed a generic

331 activation energy as suggested by MTE, with an additional possibility of random variation

332 between countries and mesocosms due to interactions and covariation with factors other than

333 those controlled for experimentally. These assumptions are justified, since country-specific

334 activation energies and absolute metabolic rates at $15^{\circ} \mathrm{C}$ did not reveal systematic changes in

335 relation to average temperature (S4). In this situation, the mixed-effect models approach that we

336 chose is reliably capable of estimating the average activation energy as well as the absolute 
metabolic rate, as validated in a simulation approach emulating the structure and random

338 structure of our experimental data (S8).

339 The same basic model as in Equation (6) was used for the analysis of GPP, daylight length-

340 corrected $\mathrm{GPP}_{\mathrm{d}}$, ER, and the ER/GPP ratio. Model selection of random and fixed effects was

341 done based on likelihood ratio tests by stepwise backward elimination ("ImerTest" package,

342 Kuznetsova et al., 2014; "step" function). However, we retained the main effects of depth,

343 nutrients, and temperature as a minimum, since we - apart from significance - sought to describe

344 effect size and to conduct comparisons between estimated and predicted values based on the

345 MTE. Effect sizes were calculated using standardized predictors following Gelman (2008).

346 Model validation was conducted by graphical inspection of the Pearson residuals, including their

347 relation to all predictor variables. The model fit was assessed by conditional (variance explained

348 by fixed effects) and marginal (variance explained by fixed and random effects) coefficients of

349 determination (“MuMIn” package, Bartoń 2015; "r.squaredGLMM” function). Treatment-

350 specific confidence intervals were computed based on a " $t$ " statistic with degrees of freedom

351 established by the Kenward \& Rogers method ("lsmeans" package, Lenth \& Hervé 2015;

352 function "lsmeans").

353

354 Covariation of temperature, depth, and nutrient levels with TP, TN, $\mathrm{K}_{\mathrm{d}}, \mathrm{MAL}, \mathrm{LP}$ eff, $\mathrm{Chl} a$, and 355 PVI was assessed with the same basic mixed effects model as in Equation (6). However, rather 356 than the scaled inverse water temperature, a centered water temperature of $15^{\circ} \mathrm{C}$ was used. Box357 Cox transformation was applied for the dependent variable to meet model assumptions ("MASS" 358 package, Ripley et al., 2015; "boxcox" function). Factor covariate interaction was probed using 359 two-tailed $t$ tests for pairwise comparisons of least-square-means over the temperature gradients 17 
360 at one degree intervals ("Ismeans" package; Lenth \& Hervé 2015). Reported treatment-wise

361 adjusted means ("phia" package, Rosario-Martinez 2015; "InteractionMeans" function) as well as

362 the direction and amount of average change between $7{ }^{\circ} \mathrm{C}$ and $29^{\circ} \mathrm{C}$ (the temperature range we

363 tested) were estimated based on these models.

364

365 Using semi-partial Spearman correlation, we assessed the differential influence of MAL, $\mathrm{PP}_{\text {eff, }}$

366 daylight length, Chl $a$, PVI, and inverse and scaled water temperatures on GPP, ER, and the

367 ER/GPP ratio (“ppcor” package, Kim, 2012 ; “spcor” function). Semi-partial Spearman

368 correlation coefficient $r$ and percentile $95 \%$ confidence intervals were bootstrapped over

369 mesocosms ("boot” package, Canty \& Ripley, 2015; "boot” and "boot.ci” function). To further

370 disentangle the influence of daylight lengths from the effect of temperature on GPP, we

371 compared the results from two separate regression models based on standardized variables,

372 conducted a residual regression analysis (see S7), and analyzed daylight length-corrected GPP

373 (Allen et al., 2005). 


\section{Results}

375 Water temperatures revealed a distinct seasonal pattern in all countries (Table 1, Figure 1a). From

376 July to November 2011, water temperature ranged from 6.8 to $29.1{ }^{\circ} \mathrm{C}$ (all countries included).

377 Water temperatures were highly correlated with monthly mean air temperatures $(r=0.88$ with a

$37895 \%$ confidence interval of $0.85-0.90$ ), confirming that the monthly point measurements in our

379 enclosures represented the overall seasonal temperature conditions (Table 1).

380 The water level decreased drastically in the two southern countries during autumn, while changes

381 were modest in the central and northern European countries (Figure 1b).

382

383 The differential monthly loading of phosphate resulted in significantly $(<0.05)$ different TP

384 levels between the high-nutrient and low-nutrient mesocosms over the entire temperature gradient

385 (Figure 2a, S3 Table 1). TN levels between deep eutrophic mesocosms and both low-nutrient

386 treatments were not significant for temperatures above $\sim 25^{\circ} \mathrm{C}$ (Figure $2 \mathrm{~b}, \mathrm{~S} 3$ Table 1). Light

387 availability, as measured by the mean available light (MAL) and the effective light period (LP eff ,

388 was highest in the shallow mesotrophic followed by the shallow eutrophic mesocosms, and the

389 deep mesotrophic mesocosms; it was lowest in the deep eutrophic mesocosms (S3 Figure 1b, c).

390 Over the entire temperature gradient, MAL differed significantly $(<0.05)$ between all treatments

391 (for DL - SH above $9{ }^{\circ} \mathrm{C}$ ). $\mathrm{LP}_{\text {eff }}$ was significantly shorter in the deep eutrophic mesocosms

392 compared to all other treatments. The deep mesotrophic mesocosm had shorter $\mathrm{LP}_{\text {eff }}$ compared

393 with the shallow mesocosms for temperatures above $15{ }^{\circ} \mathrm{C}(\mathrm{SL})$ and $21{ }^{\circ} \mathrm{C}(\mathrm{SH})$, while at no

394 point did $\mathrm{LP}_{\text {eff }}$ values differ significantly among the shallow mesocosms (S3 Figure 1, S3 Table

$3951)$.

396 
Nutrient and light effects on average metabolic rates (Hypothesis (1)) - On average, GPP and ER

398 were significantly higher in the eutrophic than in the mesotrophic systems, and significantly

399 higher in the shallow than in the deep mesocosms (Table 2, Figure 3). In line with our

400 expectations, the eutrophic shallow mesocosms with ample light had the highest metabolic rates,

401 followed by eutrophic deep systems with reduced light availability, shallow mesotrophic systems

402 with ample light, and deep mesotrophic light-reduced systems.

403

404 Temperature and interaction effects (Hypotheses (2) and (3)) - Both log-transformed GPP and

405 ER increased significantly with increasing temperatures as predicted by the Arrhenius Equations

406 (1) and (2) (Figure 4, Table 2). Contrary to our expectations, we found no significant interacting

407 effects between water temperature and the different light and nutrient regimes on GPP and ER.

408 The average temperature sensitivity of ER in all treatments was 0.62 , predicting a 13.5 -fold

409 increase in ER over a temperature range from 0 to $30^{\circ} \mathrm{C}$. The average temperature sensitivity of

410 GPP amounted to 0.54 (Figure 4, Table 3), predicting a 9.7-fold increase in GPP over a

411 temperature range from 0 to $30^{\circ} \mathrm{C}$. Thus, as predicted from the metabolic theory, ER increased

412 more with temperature than did GPP. Consequently, according to Equations (3) and (4), the

413 activation energy of the ER/GPP ratio was expected to average $0.08 \mathrm{eV}$. This corresponds to a

414 predicted 1.4-fold increase in the ratio over a temperature range from 0 to $30^{\circ} \mathrm{C}$. Although close

415 to the theoretically predicted value, the actual estimated average activation energy of $0.13 \mathrm{eV}$ for

416 the ER/GPP ratio was not significant (Figure 5a, Table 3). However, due to lower absolute

417 ER/GPP ratios in eutrophic compared with mesotrophic systems, the mesotrophic mesocosms

418 had, on average, a 10\% lower metabolic-driven carbon sequestration capacity than the eutrophic

419 mesocosms over a temperature range from 0 to $30^{\circ} \mathrm{C}$.

20 
421 The effect of covariates on metabolic rates - The average temperature sensitivity for daylight

422 length-corrected GPP had an estimated average activation energy of $0.31 \mathrm{eV}$, and was thus close

423 to the canonical temperature dependence of photosynthesis. However, it was not significant at the

4240.05 percent level (Table 2).

425 Results from semi-partial Spearman correlations confirmed the importance of temperature for

426 metabolic rates independent of light-related factors (Table 4). As expected, however, light-related

427 factors were also significantly correlated with GPP, except in shallow mesotrophic systems.

428 Significant correlations between GPP and Chl $a$ or PVI were observed in systems in which these

429 drivers were highest, i.e. in the eutrophic and mesotrophic shallow mesocosms (S3 Figure 2,

430 Table 4). In addition to temperature, ER was strongly correlated with GPP.

432 Temperature-specific switch from autotrophy to heterotrophy (Hypothesis (4)) - In the

433 mesotrophic mesocosms, based on Equation (5) and average values from the mixed effects

434 regression for GPP and ER (Table 3), the switch from autotrophy to heterotrophy generally

435 occurred at lower temperatures $\left(12{ }^{\circ} \mathrm{C}\right.$ and $\left.10^{\circ} \mathrm{C}\right)$ than in the eutrophic mesocosms $\left(21^{\circ} \mathrm{C}\right.$ and

$43619^{\circ} \mathrm{C}$ ) (Figure 5b). This is in line with our prediction. In contrast, light regime and mixing depth

437 had only a minor impact on the switch point. This is confirmed by a significant nutrient effect ( $p$

$438=0.05)$, but an insignificant depth effect $(\mathrm{p}=0.85)$ for the ER/GPP ratio (Figure 5a, Table 2).

\section{Discussion}


442 It is anticipated that climate change will affect shallow lake metabolism and thereby the ability of

443 such lakes to sequester carbon due to direct and indirect impacts on major drivers such as

444 temperature, nutrients, water level, and light conditions (Tranvik et al., 2009; Nickus et al., 2010;

445 Jeppesen et al., 2015). We confirmed the existence of a positive relationship between nutrient

446 concentrations, light availability, temperature, and metabolic rates in shallow lakes.

448 The observed apparent differential temperature sensitivity between GPP and ER adds support to

449 the anticipated shift (Cole et al., 2000; Staehr \& Sand-Jensen, 2006; Yvon-Durocher et al., 2010a,

450 2010b) towards an increasing degree of heterotrophy in shallow lake ecosystems with increasing

451 temperatures. Since the observed activation energy for ER was higher than for GPP, ecosystem

452 respiration increased more than production, leading to a predicted increase in ER/GPP over the

453 tested temperature gradient. The average temperature sensitivity of the ER/GPP ratio itself was

454 not significant, perhaps due to the inherent variance of both the GPP and the ER estimates.

455 Nonetheless, the estimated average activation energy of $0.13 \mathrm{eV}$ is in accordance with the

456 predicted values of $0.08 \mathrm{eV}$ based on equation (4) (Figure 5b). The established apparent average

457 temperature sensitivities of the metabolic rates of $0.62 \mathrm{eV}$ and $0.31 \mathrm{eV}$ for ER and GPP per

458 daylight hour, respectively, match well with predictions for the physiological temperature

459 dependence for respiration $(0.6 \mathrm{eV})$ and photosynthesis $(0.3 \mathrm{eV})$ predicted by the metabolic

460 theory (Allen et al., 2005). With a value of $0.54 \mathrm{eV}$, the observed activation energy of GPP per

461 day exceeds the predicted physiological temperature dependence, but closely conforms to

462 findings from other aquatic environments: $0.50 \pm 0.18 \mathrm{eV}$ (Wilken et al., 2013); $0.54 \pm 0.24 \mathrm{eV}$

463 (Demars et al., 2011); and $0.45 \mathrm{eV}$ (95\% CI 0.38-0.53) (Yvon-Durocher et al., 2010b). 
464 Most algae and cyanobacteria have carbon-concentration mechanisms (CCMs) to prevent the

465 oxygenase activity of Rubisco, particularly under low $\mathrm{pCO}_{2}$ and high alkalinity conditions

466 (Raven et al. 2011; Falkowski \& Raven, 2013; Demars et al., 2016). CCMs are assumed to be the 467 cause of the systematic higher activation energy for GPP found in freshwater systems compared 468 with the activation energy derived for terrestrial C3 plants (Demars et al. 2015, 2016). The

469 alkalinity in our systems was at intermediate levels on average (mean $1363 \pm 27 \mathrm{~mol} \mathrm{~L}^{-1}$ eq $\mathrm{Hcl}$ ).

$470 \mathrm{pCO}_{2}$ levels, estimated from midday alkalinity and daily average $\mathrm{pH}$ (Trolle et al., 2012), indicate

471 the potential of low $\mathrm{pCO}_{2}$ episodes (mean epCO2 $\approx 0.55 \pm 0.07$ times atmospheric pressure),

472 making the active operation of CCMs likely. However, in our systems, high positive correlations

473 between daylight length and temperature serve as a reasonable explanation for the higher-than-

474 expected activation energy, as daylight length-corrected estimates led to the physiologically

475 predicted temperature sensitivity of $0.31 \mathrm{eV}$. Interestingly, regions with seasonal temperature

476 fluctuations exhibit a natural correlation between daylight length and temperature, possibly

477 mitigating the expected shift towards heterotrophy.

478

479

480 The expected temperature at which a system switches from autotrophy to heterotrophy can be

481 modeled in the framework of MTE according to Equation 5. The model suggests that the

482 temperature threshold at which a system switches from net autotrophic to net heterotrophic

483 depends on the extent of the differential temperature sensitivity between GPP and ER ( $E_{\mathrm{GPP}}$ and

$484 \mathrm{E}_{\mathrm{ER}}$, respectively) and on the $\log$ ratio between GPP and ER at a reference temperature, Tc (here,

$48515^{\circ} \mathrm{C}$ ). This temperature threshold turned out to be affected by trophic state (Figure 5a): higher

486 nutrient availability in the eutrophic mesocosms led not only to significantly higher GPP and ER, 23 
487 but also to significantly lower ER to GPP ratios $(0.9(\mathrm{DH})$ and $0.91(\mathrm{SH}))$ than under mesotrophic 488 conditions (0.98 (DL) and 0.99 (SL)). This apparently small difference was, however, large 489 enough to cause an average temperature threshold increase of approximately $5{ }^{\circ} \mathrm{C}$ (based on 490 ER/GPP ratio). Thus, under the predicted warming scenarios of 1.5 to $5{ }^{\circ} \mathrm{C}$ by 2100 (Rogelj et al., 491 2012; Stocker et al., 2013), high-nutrient systems are likely to have a lower risk of becoming net 492 heterotrophic than systems with lower nutrient concentrations (Figure 5b, Table 3). The direct 493 effect of water depth on the ER/GPP ratio was not significant, and the effect of depth on the 494 threshold temperature was low, indicating that a reduced water level is of minor importance for 495 the switch from autotrophy to heterotrophy in these generally shallow systems.

497 In our experiment the confirmation of the MTE was strong when aggregating data from all 498 countries and seasons, while at the same time, temperature sensitivity exhibited a relatively high 499 idiosyncrasy between countries (S4 Table 2). This is in line with findings from other studies, 500 where single systems tend to deviate from the MTE predictions (De Castro \& Gaedke, 2008, 501 Davidson et al., 2015), while larger-scale studies are often in good agreement with the predictions 502 (López-Urrutia et al., 2006, Yvon-Durocher et al., 2012). Potential mechanisms behind this 503 variation include acclimatization and adaptation processes, which are hypothesized to induce 504 reduced temperature sensitivity with increasing average temperature (Atkin \& Tjoelker, 2003; 505 Hikosaka et al., 2006; Hartley et al., 2008; Angilletta, 2009; Smith \& Dukes, 2012). In this study, 506 we found no evidence for a systematic change in activation energy with average temperature (S4 507 Table 2), which is in line with Perkins et al. (2012), who reported consistent Q10 temperature 508 coefficient values for ER regardless of the thermal history or community composition of 
509 biofilms, as well as with a global survey of activation energies based on satellite data by Kraemer

510 et al. (2016).

511 Furthermore, contrary to our expectations, we observed no significant interactions between the

512 temperature sensitivity of both GPP and ER and nutrient levels, or depth. We can only speculate

513 about the underlying mechanisms. Perhaps the missing depth-temperature interaction reflects

514 light adaptation, rendering photosynthesis primarily dependent on the maximum photosynthetic

515 rate. The lack of TP-temperature interaction indicates that either TP affinity was not a function of

516 temperature, or that shifts in community composition in the mesotrophic mesocosms towards

517 species with higher phosphate affinity prevented limitation of photosynthesis by phosphorus

518 (Domis et al., 2014). However, lack of sensitivity to depth and nutrients may also reflect the

519 relatively modest variations in these experimental variables.

520 Therefore, a better understanding of the factors leading to deviations from MTE-predicted

521 temperature sensitivity remains an important area of research.

522

523 A decline in water level, as already reported and further anticipated within the context of global

524 warming for lakes in the Mediterranean region (Coops et al., 2003; Beklioglu et al., 2006, 2007;

525 Jeppesen et al., 2015), affects mixing depth and light availability. We found a significantly lower

526 GPP and ER in deep than in shallow mesocosms (Table 3). The difference in production levels

527 was most likely generated by the influence of depth on the light availability, while impacts on gas

528 exchange due to a lower surface-to-volume ratio were most likely negligible since all mesocosms

529 were fully mixed. Light saturation for photosynthesis is specific to each algal species and ranges

530 from around 60 to $100 \mu \mathrm{mol} \mathrm{m} \mathrm{s}^{-1}$ (Lampert \& Sommer, 1999). This confines deep mesocosms

531 to the lower end of the range, and makes them more prone to being light-limited, while the 25 
532 shallow mesocosms are close to, or above, the upper end of the range (S3 Table 1), and thus most

533 likely light-saturated. Additionally, only in the eutrophic deep systems was the sediment layer

534 generally below the euphotic zone; thus, only these mesocosms had a considerably shorter

535 average $\mathrm{LP}_{\text {eff }}$ compared with all other treatments (S3 Table 1). Therefore, a reduction in water

536 level considerably improves light availability, and might allow benthic primary production where

537 it was not possible before.

538

539 The linear mixed effect regression approach is an optimal method for analyzing our data as long

540 as we can reasonably assume a generic temperature sensitivity of metabolic rates or random

541 variation in temperature sensitivity due to interactions with factors randomly varying between

542 countries (see Supplement S8). Since the between-country comparison of systematic changes in

543 temperature sensitivity with average temperature indicated no systematic change (see Supplement

544 S4), there is solid justification for the approach used in this study. Furthermore, this approach

545 would be sensitive to interactions between average temperature sensitivity and nutrients, or

546 between average temperature sensitivity and water level. However, the use of this approach also

547 implies that we must analyze temperature sensitivity, as it responds to seasonal temperature

548 changes, as opposed to controlled experimental temperature manipulation. The temperature

549 response of ecosystem level metabolic rates based on seasonal data captures the apparent

550 temperature sensitivity towards relatively short-termed temperature changes and cannot replace a

551 true experimental test of the effect of global warming (therefore, we use "apparent" temperature

552 sensitivity). This is a limitation, but the response to seasonal temperature changes is of scientific

553 interest, since seasonal temperature changes are the dimension along which the property of

554 temperature sensitivity takes effect in ecosystems. Like all experimental approaches, mesocosm 26 
experiments come with inherent abstractions from the natural complexity, as well as their own challenges, which restrict a direct generalization of results to natural systems. In our experimental design, the constant mixing by aquarium pumps creates ideal constant mixing conditions, which prevents the natural variability in mixing intensity, including micro- and short-term stratification events. Mixing-induced fluctuation in light conditions has been shown to influence phytoplankton growth rates (Shatwell et al. 2012, Köhler et al. 2017), and stratification influences the availability of nutrients and oxygen (Wilhelm \& Adrian, 2008). In turn, phytoplankton growth impacts water transparency and thus water temperature and the mixing regime (Shatwell et al. 2016). However, differential warming of our mesocosms due to differences in water transparency was prevented, since water temperature in the mesocosms was mainly determined by the surrounding lake. Another well-known general problem in mesocosm studies is periphyton growth on the walls of the enclosures, forming in part a micro-environment. There is limited knowledge about the influence of periphyton on nutrient cycling and metabolic rates in the open water column, which prevents quantification (Wetzel, 2001; Petersen, 2009). Furthermore, our experimental design may have influenced the proportion of GPP to ER, as we included sediment, which contained foreign organic matter that may have enhanced ER at higher temperatures and, thus, the ratio. While this may potentially affect the absolute values (if not in equilibrium with the current conditions in the mesocosm) of thresholds regarding the shift to heterotrophy, it does not affect the observed direction of changes and the overall conclusions. However, the absolute thresholds should be interpreted with caution.

Research indicates that shallow lakes play an important role in local and global carbon cycling, as they are the most numerous type of lake in the world (Cael et al., 2017; Tranvik et al., 2009). 27 
578 Given that a differential temperature sensitivity of ER and GPP poses a potential feedback

579 mechanism to atmospheric $\mathrm{CO}_{2}$ levels in a warming scenario, understanding the metabolic

580 processes of shallow lake ecosystems, and how they will be affected by a changing climate, is not

581 only of basic but also of applied ecological interest. The results of this study confirmed and

582 quantified the varying apparent temperature sensitivity of GPP and ER and showed that trophic

583 state is important for the question of how much warming a shallow lake system can tolerate

584 before it switches from net autotrophy to net heterotrophy.

585 We linked our experimental findings with the framework of the MTE and tested theoretically

586 derived predictions on our data. In line with earlier studies, we found good agreement between

587 theory and practice, which affirmed the potential of the MTE also in the context of shallow lakes.

588 Furthermore, we found that the balance between ER and GPP depends not only on the energy

589 supply, as in the MTE, but also on the availability of nitrogen and phosphorus. Thus, we

590 conclude that quantitative inclusion of these nutrients in the MTE, as suggested for instance by

591 Allen \& Gillooly, 2009, Anderson-Teixeira \& Vitousek, 2012 and Davidson et al., 2012, could

592 greatly add to its predictive power for shallow lakes. 


\section{Acknowledgements}

594 We thank the technical staff at the various experimental sites for their support. We thank Alena S.

595 Gesell, Deniz Özkundakci, Jan-Hendrik Schleimer, Silke Schmidt, Torsten Seltmann, and Tom

596 Shatwell for their helpful discussions during the preparation of this manuscript. We thank Anne

597 Mette Poulsen, Adam Wilkins, and Michael Thayne for their valuable editing of the manuscript.

598 We are also grateful to reviewers for their valuable comments on the manuscript. This study was

599 supported by FP-7 REFRESH (Adaptive strategies to Mitigate the Impacts of Climate Change on

600 European Freshwater Ecosystems, Contract No.: 244121) and the MARS project (Managing

601 Aquatic ecosystems and water Resources under multiple Stress), funded under the 7th EU

602 Framework Programme, Theme 6 (Environment including Climate Change), Contract No.:

603603378 (http://www.mars-project.eu), TUBITAK- ÇAYDAG (projects no.: 105Y332 and

604 110Y125), the Middle East Technical University (METU)-BAP program of Turkey. EJ was

605 further supported by AU Centre for Water Technology and AİÇ was also supported by

606 TUBITAK (project 296 nos.: 105 Y332 and 110Y125). 


\section{References}

608

609 Allen, A. P., J. F. Gillooly, and J. H. Brown. 2005. Linking the global carbon cycle to

$610 \quad$ individual metabolism. Funct. ecol. 19: 202-213.

611 Allen, A. P., and Gillooly. 2009. Towards an integration of ecological stoichiometry and the

612 metabolic theory of ecology to better understand nutrient cycling. Ecol. let. 12: 369-384.

613 Anderson-Teixeira, K. J., and P. M. Vitousek. 2012. Ecosystems, p. 99-111. In R. M. Sibly, J.

614 H. Brown, A. Kodric-Brown [eds.], Metabolic Ecology. Wiley-Blackwell.

615 Andersson, E., and S. Sobek. 2006. Comparison of a mass balance and an ecosystem model

616 approach when evaluating the carbon cycling in a lake ecosystem. Ambio 35: 476-483.

617 Angilletta, M. J. J. 2009. Thermal Adaptation - A Theoretical and Empirical Synthesis, Oxford $618 \quad$ University Press, Oxford.

619 Atkin, O. K., and M. G. Tjoelker. 2003. Thermal acclimation and the dynamic response of 620 plant respiration to temperature. Trends plant sci. 8: 343-51.

621 Balmer, M. B., and J. A. Downing. 2011. Carbon dioxide concentrations in eutrophic lakes:

622 undersaturation implies atmospheric uptake. Inland Waters 1: 125-132.

623 Bartoń, K. 2015. MuMIn: Multi-Model Inference. R package version 1.13.4, http://cran.r624 project.org/package=MuMIn.

625 Bates, D., M. Maechler, B. Bolker, and S. Walker. 2014. 1me4: Linear mixed-effects models 626 using Eigen and S4. R package version 1.1-7, http://cran.r-project.org/package=lme4.

627 Beklioglu, M., G. Altınayar, and C. T. Tan. 2006. Water level control over submerged 628 macrophyte development in five Mediterranean Turkey. Arch. hydrobiol.: 166 (4): 535$629 \quad 556$. 
630 Beklioglu, M., S. Romo, I. Kagalou, X. Quintana, and E. Bécares. 2007. State of the art in the

631 functioning of shallow Mediterranean Lakes: workshop conclusion. Hydrobiologia 196:

$632 \quad 317-326$.

633 Berggren, M., H. Laudon, A. Jonsson, and M. Jansson. 2010. Nutrient constraints on

634 metabolism affect the temperature regulation of aquatic bacterial growth efficiency.

$635 \quad$ Microb. ecol. 60: 894-902.

636 Bernacchi, C. J., E. L. Singsaas, C. Pimentel, A. R. Portis Jr., and S. P. Long. 2001. Improved 637 temperature response functions for models of Rubisco-limited photosynthesis. Plant cell 638 environ. 24: 253-259.

639 Brothers, S. M., S. Hilt, K. Attermeyer, H. P. Grossart, S. Kosten, B. Lischke, T. Mehner, N.

640 Meyer, K. Scharnweber, and J. Köhler. 2013. A regime shift from macrophyte to

641 phytoplankton dominance enhances carbon burial in a shallow, eutrophic lake. Ecosphere

$642 \quad 4: 1-17$.

643 Brown, J.H., J. F. Gillooly J. F., A. P. Allen, V. M. Savage, and G. B. West. 2004. Toward a 644 metabolic theory of ecology. Ecology 85: 1771-1789.

645 Cael, B.B., A.J. Heathcoate and D.A. Seekell. 2017. The volume and maen depth of Earth's $646 \quad$ lakes. Geophy. Res. Lett. 44: 209-218.

647 Canty, A., and B. Ripley. 2015. boot: Bootstrap R (S-Plus) Functions. R package version 1.3$648 \quad 15$, http://cran.r-project.org/package=boot.

649 De Castro, F., and U. Gaedke. 2008. The metabolism of lake plankton does not support the $650 \quad$ metabolic theory of ecology. Oikos 117: 1218-1226. 

heterotrophy in lakes during nutrient addition and food web manipulations. Limnol. oceanogr. 45: 1718-1730. Duarte, P. Kortelainen, J. A. Downing, J. J. Middelburg, and J. Melack. 2007. Pluming the global carbon cycle: Integrating Inland waters into the terrestrial carbon budget. Ecosystems 10: 172-185. ecosystem metabolism with high frequency data. Ecosystems 14: 935-948.

Coops, H., M. Beklioglu, and T. L. Crisman. 2003. The role of water-level fluctuations in shallow lake ecosystems - workshop conclusions. Hydrobiologia 506: 23-27. temperature and nutrients across levels of ecological organization. Glob. chang. biol. 21: $1025-1040$.

672 Davidson, E. A., S. Samanta, S. C. Samantha, and K. Savage. 2012. The Dual Arrhenius and Michaelis-Menten kinetics model for decomposition of soil organic matter at hourly to seasonal time scales. Glob. chang. biol. 18: 371-384.

Davidson, T. A., J. Audet, J.-C. Svenning, T. L. Lauridsen, M. Søndergaard, F. Landkildehus, S. E. Larsen, and E. Jeppesen. 2015. Eutrophication effects on greenhouse gas fluxes from shallow-lake mesocosms override those of climate warming. Glob. chang. biol. 21: 44494463.

Del Giorgio, P. A., and R. H. Peters. 1994. Patterns in planktonic P:R ratios in lakes: Influence 32 of lake trophy and dissolved organic carbon. Limnol. oceanogr. 39: 772-787. 
Demars, B. O. L., J. Russell Manson, J. S. Ólafsson, G. M. Gíslason, R. Gudmundsdóttir, G. Woodward, J. Reiss, D. E. Pichler, J. J. Rasmussen, and N. Friberg. 2011. Temperature and the metabolic balance of streams. Freshwater biol. 56: 1106-1121. oxygen method: Principles, practice, and perspectives. Limnol. oceanogr., methods 13: $356-374$.

680 2003. Scaling metabolism from organisms to ecosystems. Nature 423: 639-42. lowland streams dominated by submerged macrophytes, p. 855-860. In W. K. Lauenroth, and G. V. Skogerboe [eds.], Analysis of Ecological Systems: State-of-the art in Ecological Modelling, Volume 5. Elsevier. New Jersey. 27: $2865-2873$.

Gillooly, J. F., J. H. Brown, G. B. West, V. M. Savage, and E. L. Charnov. 2001. Effects of 33 size and temperature on metabolic rate. Sci. 293: 2248-2251. 

1119. microbial respiration in arctic soil does not acclimate to temperature. Ecol. let. 11: 10921100. acclimation of photosynthesis: Mechanisms involved in the changes in temperature dependence of photosynthetic rate. J. exp. bot. 57: 291-302.

Jeppesen, E., M. Søndergaard, T. L. Lauridsen, T. A. Davidson, Z. Liu, N. Mazzeo, C. P. Nõges, J. L. Attayde, T. Zohary, J. Coppens, T. Bucak, R. F. Menezes, impacts of global warming and water abstraction on lakes and reservoirs due to changes in water level and related changes in salinity. Hydrobiologia 750: 201-227. http://cran.r-project.org/package=ppcor.

Kirk, J. T. O. 2010. Light and Photosynthesis in Aquatic Ecosystems, 3rd ed.. Cambridge 
Köhler J., L. Wang, A. Guislan and T. Shatwell. 2018. Influence of vertical mixing on lightdependency of phytoplankton growth. Limnol. oceanogr. 63: 1156-1167. Sternberg, and Marten Scheffer. 2010. Climate-dependent CO 2 emissions from lakes. Glob. biogeochem. cycles 24: 1-7. Schladow, E. Silow, L. M. Sitoki, R. Tamatamah and P. B. McIntyre. 2016. Global patterns in lake ecosystem responses to warming based on the temperature dependence of metabolism. Glob. chang. biol. 56: 1447-1455.

Kuznetsova, A., P. B. Brockhoff, and R. H. Bojesen. 2014. ImerTest: Tests in Linear Mixed Effects Models. R package version 2.0-20, http://cran.r-project.org/package=lmerTest. Papastergiadou, P. Zingel, A. I. Çakiroğlu, U. Scharfenberger, S. Drakare, T. Nõges, M. 
Lenth, R. V., and M. Hervé. 2015. Least-Squares Means. R package version 2.16, http://cran.r-

$743 \quad$ project.org/package $=1$ smeans

744 Liboriussen, L., T. L. Lauridsen, M. Søndergaard, F. Landkildehus, M. Søndergaard, S. E. Larsen, and E. Jeppesen. 2011. Effects of warming and nutrients on sediment community respiration in shallow lakes: an outdoor mesocosm experiment. Freshw. biol. 56: 437-447. balance of the oceans. PNAS 103: 8739-8744.

De Matos, M. P., A. C. Borges, A. T. de Matos, E. F. da Silva, and M. A. Martinez. 2014. Effect of time-temperature binomial in obtaining biochemical oxygen demand of different wastewaters. Eng. agric. 34: 332-340.

McFeeters, B. J., and P. C. Frost. 2011. Temperature and the effects of elemental food quality on Daphnia. Freshw. biol. 56: 1447-1455.

Moss, B. 2010. Climate change, nutrient pollution and the bargain of Dr Faustus. Freshw. biol.

Nickus, U., K. Bishop, M. Erlandsson, C. D. Evans, M. Forsius, H. Laudon, D. M. Livingstone, D. Monteith, and H. Thies. 2010. Direct Impacts of climate change on Freshwater Ecosystems, p. 38-64. In M. Kernan, R. W. Battarbee and B. Moss [eds.], Climate Change Impacts on Freshwater Ecosystems. Wiley-Blackwell. Waters 4: 41-48. Trimmer, and G. Woodward. 2012. Consistent temperature dependence of respiration across ecosystems contrasting in thermal history. Glob. chang. biol. 18: 1300-1311. 
Petersen, J. 2009. Enclosed Experimental Ecosystems and Scale: Tools for Understanding and Managing Coastal Ecosystems. Springer.

R Core Team. 2015. R: A Language and Environment for Statistical Computing. http://www.R-project.org/.

Raven, J. A., and M. Giordano, J. Beardall, S. C. Maberly. 2011. Algal and aquatic plant carbon concentration mechanisms in relation to environmental change. Photosyn. res. 109: $281-296$.

Ripley, B., B. Venables, D. M. Bates, K. Hornik, A. Gebhardt, and D. Firth. 2015 MASS: Modern Applied Statistics with S. R package version 7.3-40, http://cran.rproject.org/package $=$ MASS .

Rogelj, J., M. Meinshausen, and R. Knutti. 2012. Global warming under old and new scenarios using IPCC climate sensitivity range estimates. Nat. clim. chang. 2: 248-253.

De Rosario-Martinez, H. 2015. phia: Post-Hoc Interaction Analysis. R package version 0.2-0, http://cran.r-project.org/package=phia.

Sadro, S., J. M. Melack, and S. MacIntyre. 2011. Spatial and temporal variability in the ecosystem metabolism of a high-elevation lake: Integrating benthic and pelagic habitats. Ecosystems 14: 1123-1140.

de Senerpont Domis, L. N., D. B. van de Waal, N. R. Helmsing, E. van Donk, and W. M. Mooij. 2014. Community stoichiometry in a changing world: combined effects of warming and eutrophication on phytoplankton dynamics. Ecology 95: 1485-1495.

Shatwell, T., A. Nicklisch, and J. Köhler. 2012. Temperature and photoperiod effects on phytoplankton growing under simulated mixed layer light fluctuations. Limnol. oceanogr. 57: 541-553. 

dimictic regime shifts in temperate lakes. Sci. Rep. 6: 24361.

Smith, N. G., and J. S. Dukes. 2012. Plant respiration and photosynthesis in global-scale models: Incorporating acclimation to temperature and $\mathrm{CO}_{2}$. Glob. chang. biol. 19: 45-63. respiration: Drivers of daily variability and background respiration in lakes around the globe. Limnol. oceanogr. 58: 849-866.

Staehr, P. A., and K. Sand-Jensen. 2006. Seasonal changes in temperature and nutrient control of photosynthesis, respiration and growth of natural phytoplankton communities. Freshw. biol. 51: 249-262. metabolism and net heterotrophy in contrasting lakes. Limnol. oceanogr. 55: 817-830. Assessment Report of the Intergovernmental Panel on Climate Change.

Streeter, H. W., and E. B. Phelps. 1925. A study of the natural purification of the Ohio River. Public Health Bulletin 146: U.S. Public Health Service, Washington DC, 1925. 
810 Tranvik, L. J., J. A. Downing, J. B. Cotner, S. A. Loiselle, R. G. Striegl, T. J. Ballatore, P.

811 Dillon, K. Finlay, K. Fortino, L. B. Knoll, P. L. Kortelainen, T. Kutser, S. Larsen, I.

812 Laurion, D. M. Leech, S. L. McCallister, D. M. McKnight, J. M. Melack, E. Overholt, J. A.

813 Porter, Y. Prairie, W. H. Renwick, F. Roland, B. S. Sherman, D. W. Schindler, S. Sobek, A.

814 Tremblay, M. J. Vanni, A. M. Verschoor, E. von Wachenfeldt, and G. A. Weyhenmeyer.

815 2009. Lakes and reservoirs as regulators of carbon cycling and climate. Limnol. oceanogr.

$816 \quad 54: 2298-2314$.

817 Trolle, D., P. A. Staehr, T. A. Davidson, R. Bjerring, T. L. Lauridsen, M. Søndergaard, and E.

818 Jeppesen. 2012. Seasonal dynamics of $\mathrm{CO}_{2}$ flux across the surface of shallow temperate

$819 \quad$ lakes. Ecosystems 15: 336-347.

820 Welter, J. R., P. B. Jonathan, W. F. Cross, J. M. Hood, A. D. Huryn, P. W. Johnson, and T. J.

821 Williamson. 2015. Does $\mathrm{N}_{2}$ fixation amplify the temperature dependence of ecosystem

822 metabolism? Ecology 93: 603-610.

823 Wetzel, R. G. 2001. Limnology: Lake and river ecosystems, 3rd ed. Academic Press.

824 Weyhenmeyer, G. A., S. Kosten, M. B. Wallin, L. J. Tranvik, E. Jeppesen, and R. Fabio. 2015.

825 Significant fraction of $\mathrm{CO} 2$ emissions from boreal lakes derived from hydrologic inorganic

$826 \quad$ carbon inputs. Nat. geosci. 8: 933-936.

827 Wilhelm, S., and R. Adrian. 2008. Impact of summer warming on the thermal characteristics

828 of a polymictic lake and consequences for oxygen, nutrients and phytoplankton. Freshw.

829 biol. 53: 226-237.

830 Wilken, S., J. Huisman, S. Naus-Wiezer, and E. van Donk. 2013. Mixotrophic organisms

831 become more heterotrophic with rising temperature. Ecol. lett. 16: 225-233. 
832 Wykoff, D. D., J. P. Davies, A. Melis, A. R. Grossman. 1998. The regulation of

833 photosynthetic electron transport during nutrient deprivation in Chlamydomonas

$834 \quad$ reinhardtii. Plant physiol. 117: 129-139.

835 Yvon-Durocher, G., A. P. Allen, J. M. Montoya, M. Trimmer, and G. Woodward. 2010a. The

836 temperature dependence of the carbon cycle in aquatic ecosystems, pp. 267-313. In G.

837 Woodward [ed.], Advances in Ecological Research, Vol. 43. Elsevier.

838 Yvon-Durocher, G., J. I. Jones, M. Trimmer, G. Woodward, and J. M. Montoya. 2010b.

839 Warming alters the metabolic balance of ecosystems. Philos. trans. - R. Soc., Biol. Sci. 365:

$840 \quad 2117-26$.

841 Yvon-Durocher, G., J. M. Caffrey, A. Cescatti, M. Dossena, P. del Giorgio, J. M. Gasol, J. M.

842 Montoya, J. Pumpanen, P. A. Staehr, M. Trimmer, G. Woodward, and A. P. Allen. 2012.

843 Reconciling the temperature dependence of respiration across timescales and ecosystem

$844 \quad$ types. Nature 487: 472-476.

845

846 


\section{Figure legends}

2 Figure 1: a) Development of water temperature and b) change in water level due to evaporation

3 losses and precipitation gains over the experimental period from June to November by country.

$4 \mathrm{SE}=$ Sweden, $\mathrm{EE}=$ Estonia, $\mathrm{CZ}=$ Czech Republic, $\mathrm{GE}=$ Germany, $\mathrm{TR}=$ Turkey, $\mathrm{GR}=$ Greece.

6 Figure 2: Covariation of nutrients with water temperature. Covariation of monthly a) total

7 phosphorus (TP) and b) total nitrogen (TN) levels with water temperature by treatment. Main

8 images show treatment-specific least-square means over the temperature gradient with $95 \%$

9 confidence intervals at the scale of the transformed variable. The insets depict TP and TN at

10 original scale with treatment-specific average TP or TN concentrations as estimated by mixed

11 effects regression (S3 Table 1). $\mathrm{DH}=$ deep high nutrient, $\mathrm{SH}=$ shallow high nutrient, $\mathrm{DL}=$ deep

12 low nutrient and $\mathrm{SL}=$ shallow low nutrient treatment.

14 Figure 3: Treatment-wise metabolic rates at $15{ }^{\circ} \mathrm{C}$ as estimated by mixed effects regression.

15 Values were back transformed to original scale, depicting the geometric mean (Table 3). Error

16 bars represent the $95 \%$ confidence interval of the geometric mean. $\mathrm{DH}=$ deep high nutrient, $\mathrm{SH}=$

17 shallow high nutrient, $\mathrm{DL}=$ deep low nutrient and $\mathrm{SL}=$ shallow low nutrient treatment.

19 Figure 4: Arrhenius plot of GPP and ER for each treatment $(\mathrm{DH}=$ deep high nutrient, $\mathrm{SH}=$

20 shallow high nutrient, $\mathrm{DL}=$ deep low nutrient and $\mathrm{SL}=$ shallow low nutrient). The plot is based

21 on monthly measurements from July to November along a temperature gradient from Sweden to

22 Greece. The solid line is the estimated average GPP; the dotted line is the average ER as

23 estimated by mixed effects regression. Note that the actual units of the x-axis of the Arrhenius 
24 plot are $1 / \mathrm{k}\left(1 / \mathrm{T}_{\mathrm{c}}-1 / \mathrm{T}\right)$ in units of electron volts and a reference temperature, $\mathrm{T}_{\mathrm{c}}$, of $15^{\circ} \mathrm{C}$; for

25 easier interpretation, corresponding temperatures in degrees Celsius are depicted.

27 Figure 5: Water temperature-dependent switch from net autotrophy to net heterotrophy. a)

28 Average treatment-specific change in ER/GPP ratio over the temperature gradient as estimated by

29 mixed effect model (Table 3). b) Theoretically predicted switch point temperatures from

30 autotrophy to heterotrophy depending on the ER/GPP ratio at a reference temperature of $15^{\circ} \mathrm{C}$

31 (Equation 5). The solid line depicts the switch point temperature for activation energies of 0.54

$32 \mathrm{eV}$ and $0.62 \mathrm{eV}$ for GPP and ER, respectively. The dashed line represents the relation at an

33 activation energy of $0.31 \mathrm{eV}$ for GPP, as suggested by the metabolic theory of ecology and

34 established as the average apparent activation energy for daylight length-corrected primary

35 production. Superimposed are the treatment-wise average switch point temperatures as

36 established by mixed effects regression (Table 3 ). $\mathrm{DH}=$ deep high nutrient, $\mathrm{SH}=$ shallow high

37 nutrient, $\mathrm{DL}=$ deep low nutrient and $\mathrm{SL}=$ shallow low nutrient treatment. 

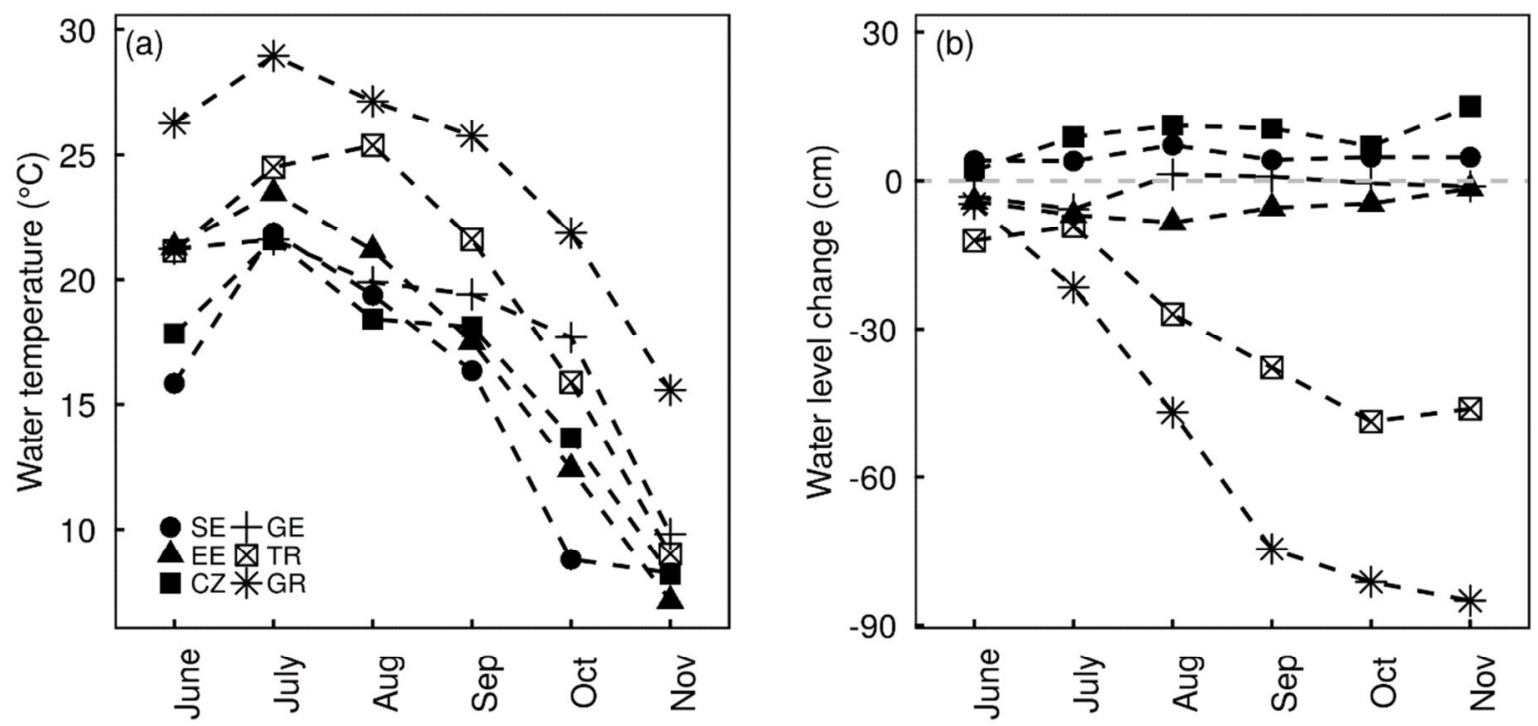

39

40

$41 \quad$ Figure 1

42 

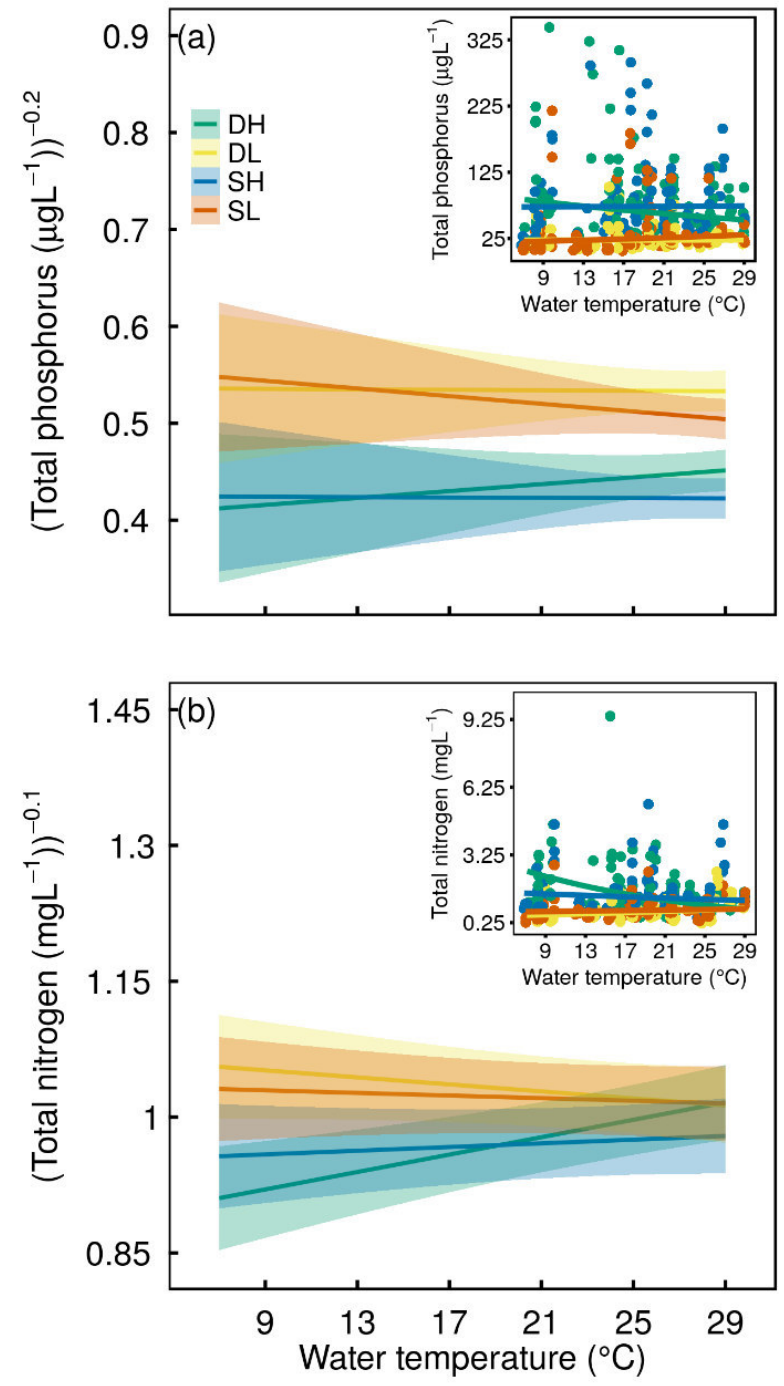

44

$45 \quad$ Figure 2

46 


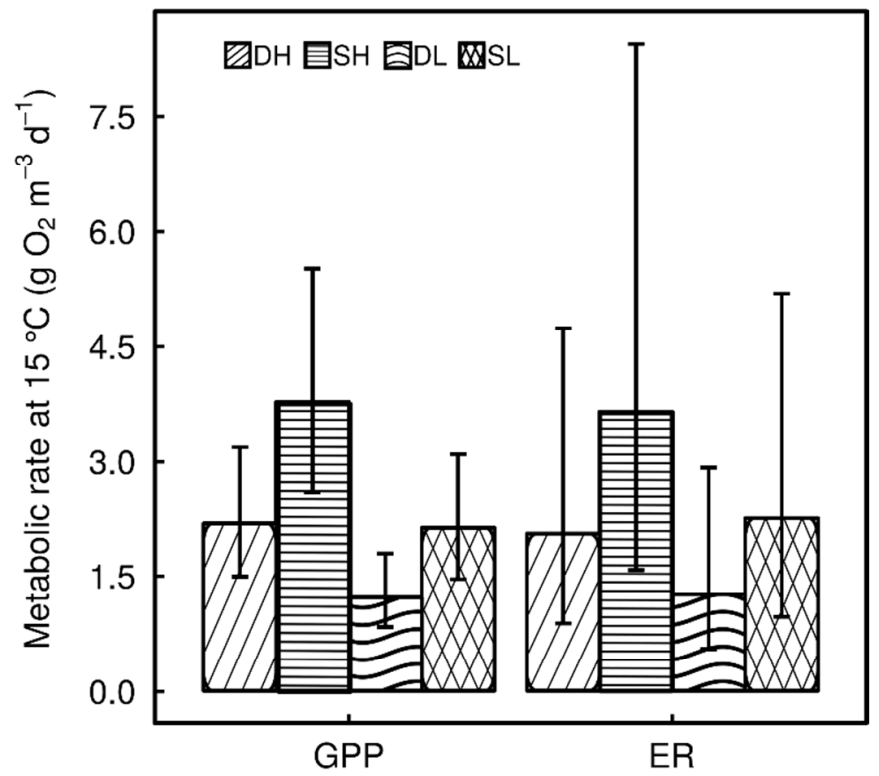

47

$48 \quad$ Figure 3

49 


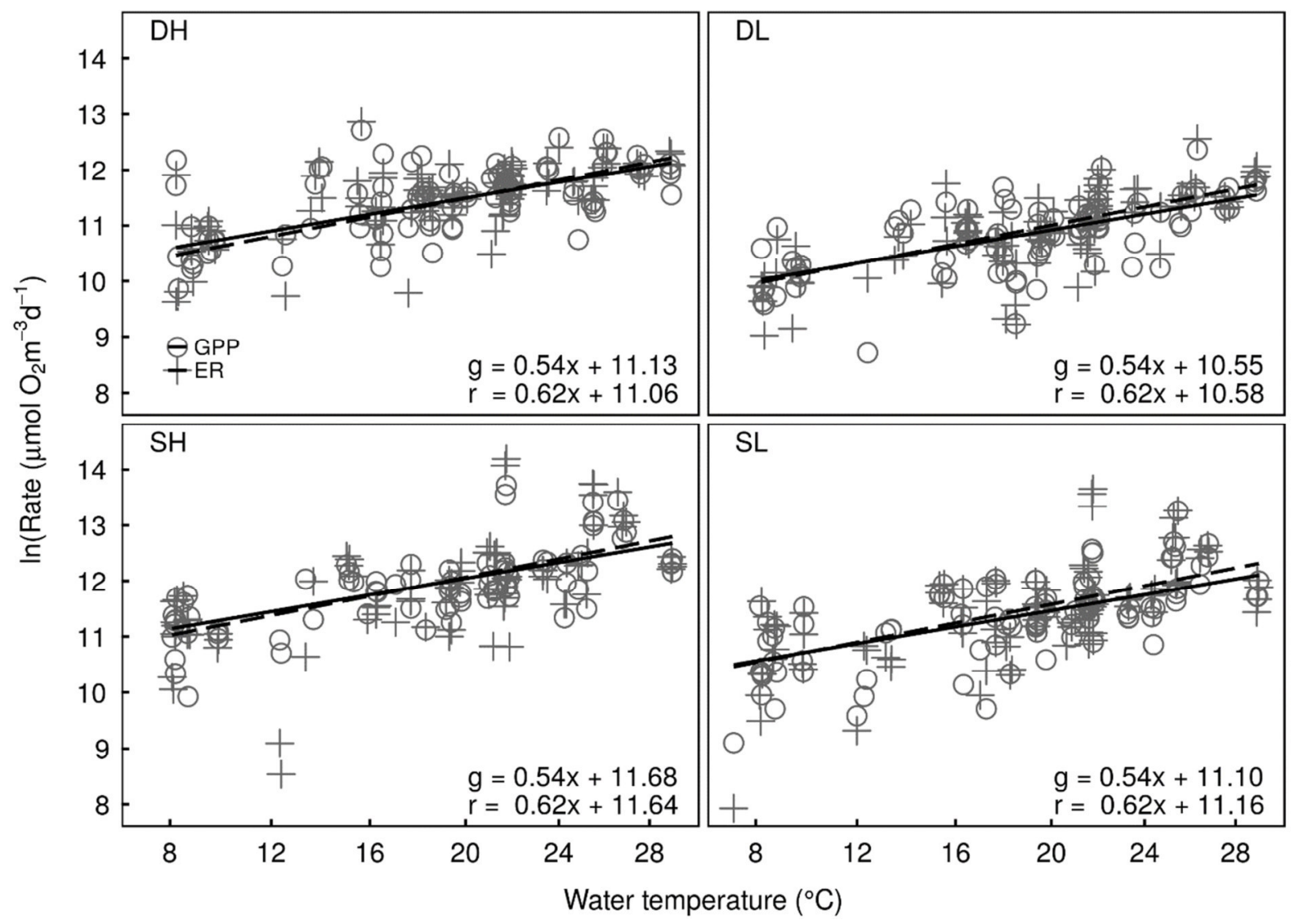

50

51 Figure 4

52 

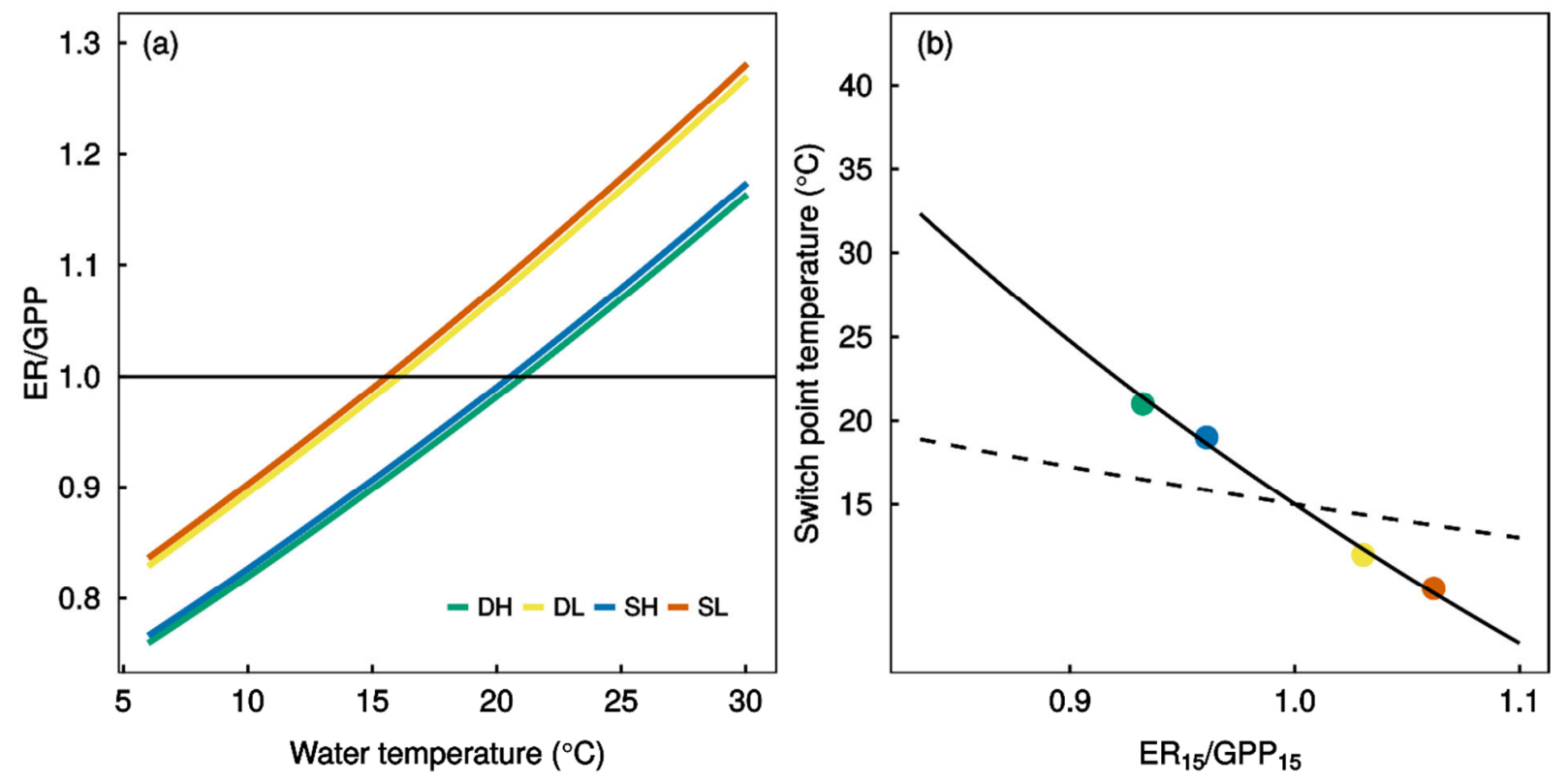

$54 \quad$ Figure 5 
1 Table 1: Location of experimental sites and average temperatures. Average air temperatures were

2 calculated based on daily average air temperatures of the periods leading up to the 24-hour

3 measurements as defined in S1 Table 1. Water temperatures are daily averages based on 24-hour

4 point measurements (S1 Table 1).

\begin{tabular}{|c|c|c|c|c|c|c|c|c|}
\hline Experimental site & Coordinates & $\begin{array}{l}\text { Altitude } \\
\text { (m a.s.l.) }\end{array}$ & & $\begin{array}{l}\text { temper } \\
\left({ }^{\circ} \mathrm{C}\right)\end{array}$ & & & $\begin{array}{l}\text { ter temp } \\
\qquad\left({ }^{\circ} \mathrm{C}\right)\end{array}$ & rature \\
\hline & & & Mean & Min & Max & Mean & Min & Max \\
\hline $\begin{array}{l}\text { Sweden (SE) - } \\
\text { Erken }\end{array}$ & $\begin{array}{l}59^{\circ} 49^{\prime} 59^{\prime \prime} \mathrm{N} \\
18^{\circ} 33^{\prime} 55^{\prime \prime} \mathrm{E}\end{array}$ & 11 & 14.5 & 8.7 & 18.8 & 14.9 & 8.1 & 22.0 \\
\hline $\begin{array}{l}\text { Estonia (EE) - } \\
\text { Võrtsjärv }\end{array}$ & $\begin{array}{l}58^{\circ} 12^{\prime} 17^{\prime \prime} \mathrm{N} \\
26^{\circ} 06^{\prime} 16^{\prime \prime} \mathrm{E}\end{array}$ & 35 & 15.1 & 7.5 & 19.9 & 16.3 & 6.8 & 24.0 \\
\hline $\begin{array}{l}\text { Germany (GE) - } \\
\text { Müggelsee }\end{array}$ & $\begin{array}{l}52^{\circ} 26^{\prime} 0^{\prime \prime} \mathrm{N} \\
13^{\circ} 39^{\prime} 0^{\prime \prime} \mathrm{E}\end{array}$ & 32 & 16.0 & 9.6 & 18.4 & 17.7 & 9.7 & 21.7 \\
\hline $\begin{array}{l}\text { Czech Republic } \\
\text { (CZ) - Vodňany }\end{array}$ & $\begin{array}{l}49^{\circ} 09^{\prime} 14^{\prime \prime} \mathrm{N} \\
14^{\circ} 10^{\prime} 11^{\prime \prime} \mathrm{E}\end{array}$ & 395 & 15.2 & 7.5 & 18.8 & 16.0 & 8.1 & 22.0 \\
\hline $\begin{array}{l}\text { Turkey (TR) - } \\
\text { ODTÜ-DSİ Gölet }\end{array}$ & $\begin{array}{l}39^{\circ} 52^{\prime} 38^{\prime \prime} \mathrm{N} \\
32^{\circ} 46^{\prime} 32^{\prime \prime} \mathrm{E}\end{array}$ & 998 & 20.0 & 10.4 & 26.2 & 19.3 & 8.2 & 25.6 \\
\hline $\begin{array}{l}\text { Greece (GR) - } \\
\text { Lysimachia }\end{array}$ & $\begin{array}{l}38^{\circ} 33^{\prime} 40^{\prime \prime} \mathrm{N} \\
21^{\circ} 22^{\prime} 10^{\prime \prime} \mathrm{E}\end{array}$ & 16 & 23.8 & 15.0 & 27.9 & 24.8 & 15.4 & 29.1 \\
\hline
\end{tabular}


5 Table 2: Results from minimal linear mixed effect regressions. The effects of inverse scaled

6 temperature (invT), depth (D) and nutrients (N), as well as their interactions, were tested on:

7 gross primary production per day (GPP), ecosystem respiration per day (ER), primary production

8 per daylight hour $\left(\mathrm{GPP}_{\mathrm{dl}}\right)$, and the ratio between ecosystem respiration and gross primary

9 production (ER/GPP). Effect size is given as regression coefficients from standardized predictors

10 (Shallow $=-0.5$, Deep $=0.5$, Low $=-0.5$, High $=0.5)$. The first $\mathrm{R}^{2}$ value refers to the marginal $\mathrm{R}^{2}$

11 (variance explained by fixed factors) and the second to the conditional $\mathrm{R}^{2}$ (variance explained by

12 fixed and random factors).

\begin{tabular}{|c|c|c|c|c|c|c|}
\hline Response & Predictor & Effect size & Std. error & T-value & P-value & $\mathrm{R}^{2}$ \\
\hline \multirow[t]{4}{*}{$\ln (\mathrm{GPP})$} & Int & 11.34 & 0.14 & 77.99 & $<0.01$ & \multirow{4}{*}{$0.50,0.8$} \\
\hline & invT & -0.91 & 0.16 & -3.29 & 0.02 & \\
\hline & $\mathrm{D}$ & -0.55 & 0.03 & -9.93 & $<0.01$ & \\
\hline & $\mathrm{N}$ & 0.58 & 0.03 & 10.52 & $<0.01$ & \\
\hline \multirow[t]{4}{*}{$\ln (\mathrm{ER})$} & Int & 11.37 & 0.32 & 34.99 & $<0.01$ & \multirow{4}{*}{$0.33,0.88$} \\
\hline & invT & -1.05 & 0.24 & -2.54 & 0.06 & \\
\hline & D & -0.58 & 0.03 & -9.37 & $<0.01$ & \\
\hline & $\mathrm{N}$ & 0.48 & 0.03 & 7.91 & $<0.01$ & \\
\hline \multirow[t]{5}{*}{$\ln \left(\mathrm{GPP}_{\mathrm{dl}}\right)$} & Int & 8.71 & 0.18 & 46.68 & $<0.01$ & \multirow{5}{*}{$10.34,0.78$} \\
\hline & invT & -0.53 & 0.15 & -2.03 & 0.10 & \\
\hline & D & -0.54 & 0.03 & -9.74 & $<0.01$ & \\
\hline & $\mathrm{N}$ & 0.58 & 0.03 & 10.49 & $<0.01$ & \\
\hline & $\mathrm{N}$ & 0.48 & 0.03 & 9.29 & $<0.01$ & \\
\hline \multirow[t]{4}{*}{$\ln (\mathrm{ER} / \mathrm{GPP})$} & Int & -0.01 & 0.14 & -0.41 & 0.71 & \multirow{4}{*}{$0.04,0.60$} \\
\hline & $\operatorname{invT}$ & -0.22 & 0.10 & -1.27 & 0.27 & \\
\hline & D & -0.01 & 0.02 & -0.20 & 0.85 & \\
\hline & $\mathrm{N}$ & -0.09 & 0.02 & -1.99 & 0.05 & \\
\hline
\end{tabular}


15 Table 3: Slope (activation energy), intercept (average metabolic rate at $15^{\circ} \mathrm{C}$ ), and temperature at which the systems switch from autotrophy to heterotrophy. Slope and intercept values are derived from minimal mixed effect models, i.e. models from which all insignificant terms are removed, but which contain at least the inverse scaled temperature and the main effects of the

19 depth and nutrient treatment (Table 2). 95\% confidence intervals are given in brackets.

20 Confidence intervals for activation energies were computed based on likelihood profiles

21 ("confint.merMod" function of the "Ime4" package). Treatment-specific confidence intervals for 22 the intercepts were computed based on t-statistics with degrees of freedom determined by the 23 Kenward \& Rogers method ("Ismeans" function of the "Ismeans" package). DH = deep high 24 nutrient, $\mathrm{SH}=$ shallow high nutrient, $\mathrm{DL}=$ deep low nutrient and $\mathrm{SL}=$ shallow low nutrient 25 treatment.

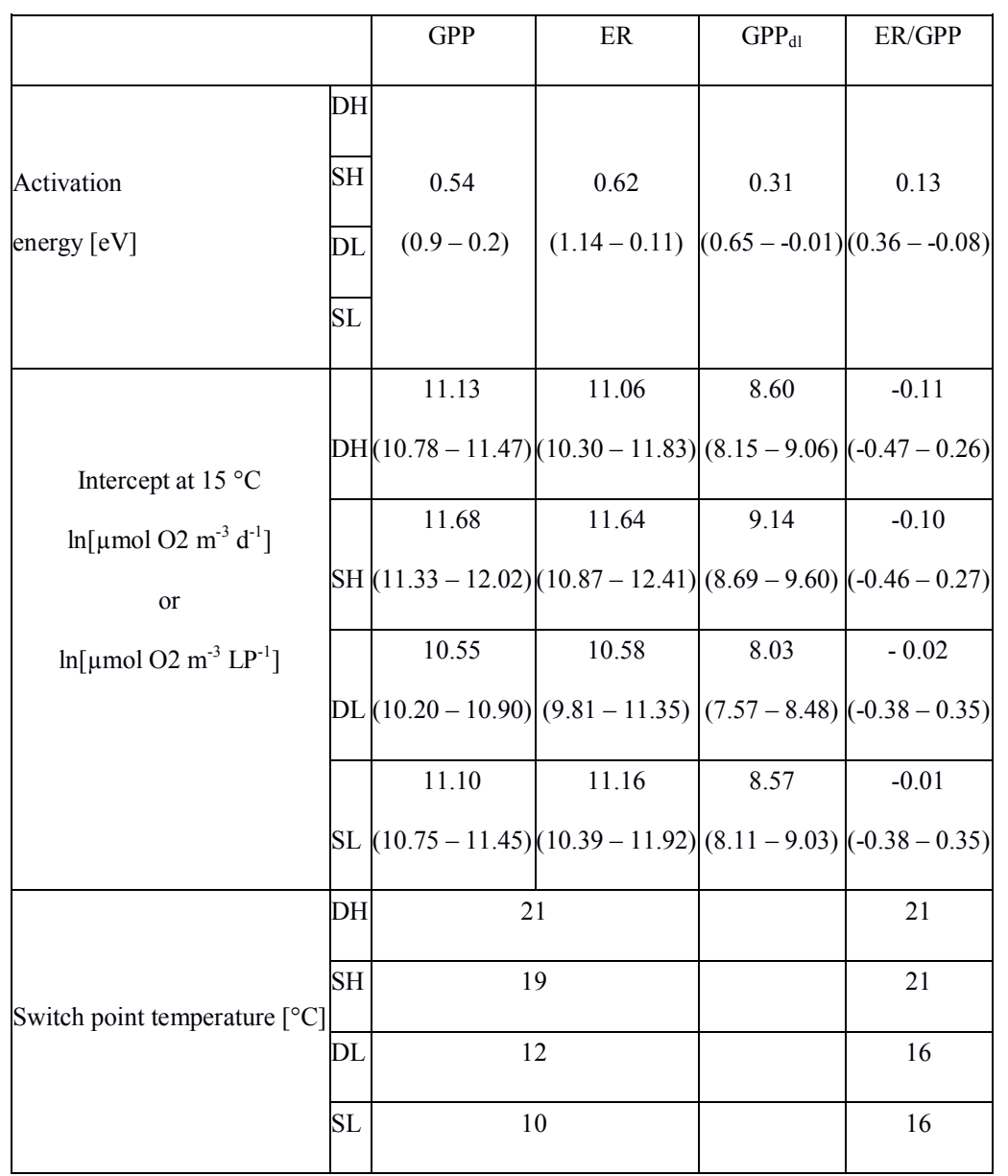


Table 4: Bootstrapped semi-partial Spearman correlation coefficient $r$ over all treatments (all)

28 and treatment-specific ( $\mathrm{S}=$ shallow, $\mathrm{D}=$ deep, $\mathrm{H}=$ high nutrient, $\mathrm{L}=$ low nutrient) for the three

29 criterion variables GPP, ER, and ER/GPP and the following predictor variables: inverse-scaled

30 temperature (invT), chlorophyll a (Chl a), PVI (plant volume inhabited), effective light period

31 (LP ${ }_{\text {eff }}$ ), mean available light (MAL), daylight length (DayL), ER/GPP ratio, GPP, and ER. Only $r$

32 values $\geq 0.10$ are reported, “*” denotes values where the $95 \%$ confidence interval did not include

33 zero, "“" denotes cells of variables not used as predictors for the particular variable.

\begin{tabular}{|c|c|c|c|c|c|c|c|c|}
\hline & & invT & Chl a & PVI & $L P_{\text {eff }}$ & MAL & DayL & GPP \\
\hline \multirow{5}{*}{ GPP } & all & $-0.23 *$ & $0.33^{*}$ & & $-0.12 *$ & $0.24 *$ & & - \\
\hline & DH & -0.22 & $0.28 *$ & & $-0.15^{*}$ & 0.16 & 0.15 & - \\
\hline & SH & $-0.40 *$ & $0.23 *$ & 0.12 & & & $0.25^{*}$ & - \\
\hline & DL & $-0.41 *$ & & & $-0.18^{*}$ & 0.13 & & - \\
\hline & SL & $-0.28 *$ & & $0.27^{*}$ & & & & - \\
\hline \multirow{5}{*}{ ER } & all & $-0.19 *$ & & & - & - & - & $0.52 *$ \\
\hline & DH & $-0.27^{*}$ & & & - & - & - & $0.43 *$ \\
\hline & SH & $-0.19 *$ & 0.1 & $0.14^{*}$ & - & - & - & $0.32 *$ \\
\hline & DL & $-0.28 *$ & & 0.12 & - & - & - & $0.38^{*}$ \\
\hline & SL & $-0.19 *$ & & & - & - & - & $0.38 *$ \\
\hline \multirow{5}{*}{ ER/GPP } & all & $-0.23 *$ & & & & & $-0.10 *$ & - \\
\hline & DH & -0.16 & -0.12 & & & -0.14 & & - \\
\hline & SH & $-0.20 *$ & & & -0.12 & & & - \\
\hline & DL & $-0.29 *$ & & & & -0.16 & -0.11 & - \\
\hline & SL & $-0.25^{*}$ & & & -0.16 & & & - \\
\hline
\end{tabular}




\section{Appendix S1}

S1 Table 1: Country-wise measurement dates for the 24-h measurement and associated month and period of analysis. Monthly 24-h measurements for measurement $3-7$ were planned to take place on 12.07, 09.08, 06.09, 04.10 and 1.11. However, the actual measurements deviated slightly from this date; thus, average values for each country, for instance monthly air temperature, are calculated over the individual period for each country leading up to a 24-h measurement.

\begin{tabular}{|c|c|c|c|c|c|c|c|c|c|c|c|c|c|}
\hline \multirow[b]{2}{*}{ Measurement no. } & \multirow[b]{2}{*}{ Associated month } & \multicolumn{2}{|c|}{ Sweden } & \multicolumn{2}{|c|}{ Estonia } & \multicolumn{2}{|c|}{ Germany } & \multicolumn{2}{|c|}{ Czech Republic } & \multicolumn{2}{|c|}{ Turkey } & \multicolumn{2}{|c|}{ Greece } \\
\hline & & Date & Period & Date II & Period & Date & Period & Date & Period & Date & Period & Date & Period \\
\hline 3 & July & $\mid \begin{array}{l}11.07 \\
12.07\end{array}$ & $14.06-11.07$ & $\begin{array}{l}12.07 \\
13.07\end{array}$ & $09.06-12.07$ & $\begin{array}{l}11.07 \\
12.07\end{array}$ & $15.06-11.07$ & $\mid \begin{array}{l}11.07 \\
12.07\end{array}$ & $14.06-11.07$ & $\begin{array}{l}12.07 \\
13.07\end{array}$ & $15.06-12.07$ & $\begin{array}{l}15.07 \\
16.07\end{array} \mid$ & $16.06-15.07$ \\
\hline 4 & August & $\mid \begin{array}{l}15.08 \\
16.08\end{array}$ & $12.07-15.08$ & $\left|\begin{array}{l}09.08 \\
10.08\end{array}\right|$ & $13.07-09.08$ & $\begin{array}{l}08.08 \\
09.08\end{array}$ & $12.07-08.08$ & 08.08 & $12.07-08.08$ & $\begin{array}{l}08.08 \\
09.08\end{array}$ & $13.07-08.08$ & $\begin{array}{l}13.08 \\
14.08\end{array} \mid$ & $16.07-13.08$ \\
\hline 5 & September & $\begin{array}{l}13.09 \\
14.09\end{array}$ & $16.08-13.09$ & $\left|\begin{array}{l}05.09 \\
06.09\end{array}\right|$ & $10.08-05.09$ & $\mid \begin{array}{l}05.09 \\
06.09\end{array}$ & $09.08-06.09$ & $\begin{array}{l}05.09 \\
06.09\end{array}$ & $09.08-05.09$ & 06.09 & $09.08-06.09$ & $\begin{array}{l}11.09 \\
12.09\end{array}$ & $14.08-11.09$ \\
\hline 6 & October & $\begin{array}{l}18.10 \\
19.10\end{array}$ & $14.09-18.10$ & $\mid \begin{array}{l}03.10 \\
04.10\end{array}$ & $06.09-03.10$ & $\begin{array}{l}04.10 \\
05.10\end{array}$ & $07.09-04.10$ & $\begin{array}{l}03.10 \\
04.10\end{array}$ & $06.09-03.10$ & 0 & $07.09-04.10$ & $\begin{array}{l}10.10 \\
11.10\end{array}$ & $12.09-10.10$ \\
\hline 7 & November & 07.11 & $19.10-07.11$ & $\begin{array}{l}31.10 \\
01.11\end{array}$ & $04.10-31.10$ & $\begin{array}{l}31.10 . \\
01.11\end{array}$ & $05.10-31.10$ & $\begin{array}{l}30.10 \\
31.10\end{array}$ & $04.10-31.10$ & $\begin{array}{l}01.11 \\
02.11\end{array}$ & $05.10-01.11$ & 08.11 & $11.10-31.10$ \\
\hline
\end{tabular}


S1 Table 2: Available data points for the statistical analysis of GPP, ER and metabolic balance (ER/GPP) and their distribution over countries, months and treatments. Imbalance in the data is due to loss of mesocosms during storm events and removal of GPP and ER estimates with standard errors larger than the estimated value or less than $5 \%$ explained variability by the model.

\begin{tabular}{|c|c|c|c|c|c|c|c|}
\hline Country & Treatment & June & July & Aug & $\mathrm{Se}$ & Oc & Total \\
\hline \multirow[t]{4}{*}{$\mathbf{S E}$} & DH & 4 & 4 & 4 & 4 & 3 & 76 \\
\hline & DL & 4 & 4 & 4 & 2 & 4 & \\
\hline & $\mathrm{SH}$ & 4 & 4 & 4 & 3 & 4 & \\
\hline & SL & 4 & 4 & 4 & 4 & 4 & \\
\hline \multirow[t]{4}{*}{ EE } & DH & 4 & 3 & 4 & 2 & 0 & 50 \\
\hline & DL & 4 & 4 & 3 & 1 & 0 & \\
\hline & $\mathrm{SH}$ & 4 & 3 & 1 & 2 & 0 & \\
\hline & SL & 4 & 4 & 3 & 3 & 1 & \\
\hline \multirow[t]{4}{*}{ GE } & DH & 2 & 2 & 2 & 2 & 2 & 60 \\
\hline & $\mathrm{DL}$ & 3 & 3 & 3 & 3 & 3 & \\
\hline & $\mathrm{SH}$ & 3 & 3 & 3 & 3 & 3 & \\
\hline & $\mathrm{SL}$ & 4 & 4 & 4 & 4 & 4 & \\
\hline \multirow[t]{4}{*}{$\mathrm{CZ}$} & $\mathrm{DH}$ & 4 & 4 & 3 & 4 & 2 & 46 \\
\hline & $\mathrm{DL}$ & 1 & 3 & 3 & 4 & 1 & \\
\hline & $\mathrm{SH}$ & 1 & 1 & 0 & 2 & 2 & \\
\hline & SL & 3 & 3 & 1 & 3 & 1 & \\
\hline \multirow[t]{4}{*}{ TR } & DH & 3 & 4 & 4 & 4 & 4 & 73 \\
\hline & DL & 2 & 3 & 4 & 4 & 3 & \\
\hline & $\mathrm{SH}$ & 3 & 4 & 4 & 4 & 4 & \\
\hline & SL & 4 & 4 & 4 & 4 & 3 & \\
\hline \multirow[t]{4}{*}{ GR } & $\mathrm{DH}$ & 4 & 4 & 4 & 4 & 4 & 69 \\
\hline & $\mathrm{DL}$ & 4 & 4 & 4 & 4 & 4 & \\
\hline & $\mathrm{SH}$ & 4 & 4 & 4 & 2 & 0 & \\
\hline & $\mathrm{SL}$ & 4 & 4 & 4 & 3 & 0 & \\
\hline Total & & 81 & 84 & 78 & 75 & 56 & 374 \\
\hline
\end{tabular}


S1 Table 3: Country- and month-wise average air temperature. For each month, column 5 shows the temperature range in regard to the warmest and the coldest country along the climate gradient, as well as the standard deviation between countries.

\begin{tabular}{|c|c|c|c|c|}
\hline Month & Country & Average air temperature $\left[{ }^{\circ} \mathbf{C}\right]$ & Monthly average air temperature $\left[{ }^{\circ} \mathrm{C}\right]$ & Range $\left[{ }^{\circ} \mathbf{C}\right] / \mathbf{S t d}\left[{ }^{\circ} \mathbf{C}\right]$ \\
\hline \multirow[t]{6}{*}{ July } & SE & 16.86 & \multirow[t]{6}{*}{20.01} & \multirow[t]{6}{*}{$9.56 / 3.52$} \\
\hline & EE & 18.75 & & \\
\hline & GE & 18.43 & & \\
\hline & $\mathrm{CZ}$ & 17.98 & & \\
\hline & TR & 21.64 & & \\
\hline & GR & 26.42 & & \\
\hline \multirow[t]{6}{*}{ August } & SE & 18.81 & \multirow[t]{6}{*}{21.29} & \multirow[t]{6}{*}{$10.11 / 4.33$} \\
\hline & $\mathrm{EE}$ & 19.92 & & \\
\hline & GE & 18.37 & & \\
\hline & $\mathrm{CZ}$ & 17.16 & & \\
\hline & TR & 26.23 & & \\
\hline & GR & 27.27 & & \\
\hline \multirow[t]{6}{*}{ September } & SE & 16.7 & \multirow[t]{6}{*}{20.18} & \multirow[t]{6}{*}{$11.51 / 4.44$} \\
\hline & $\mathrm{EE}$ & 16.35 & & \\
\hline & $\mathrm{GE}$ & 18.4 & & \\
\hline & $\mathrm{CZ}$ & 18.79 & & \\
\hline & TR & 22.99 & & \\
\hline & GR & 27.86 & & \\
\hline \multirow[t]{6}{*}{ October } & SE & 11.64 & \multirow[t]{6}{*}{16.01} & \multirow[t]{6}{*}{$11.04 / 4.04$} \\
\hline & EE & 13.2 & & \\
\hline & GE & 15.32 & & \\
\hline & $\mathrm{CZ}$ & 14.49 & & \\
\hline & TR & 18.74 & & \\
\hline & GR & 22.68 & & \\
\hline \multirow[t]{6}{*}{ November } & SE & 8.71 & \multirow[t]{6}{*}{9.78} & \multirow[t]{6}{*}{$7.50 / 2.79$} \\
\hline & EE & 7.47 & & \\
\hline & GE & 9.61 & & \\
\hline & $\mathrm{CZ}$ & 7.5 & & \\
\hline & $\mathrm{TR}$ & 10.44 & & \\
\hline & GR & 14.97 & & \\
\hline
\end{tabular}




\section{Appendix S2: Methods}

\section{S2.1 Estimation of primary production (GPP) and ecosystem respiration (ER) rates}

Temporal dynamics of dissolved oxygen were modelled according to Jeppesen et al. (2012):

$$
\frac{d D O}{d t}=K_{a, 20} \Phi^{\left(T_{t}-20\right)}\left(D O_{s a t}-O_{2}\right)+\rho \frac{I_{t}}{\eta+I_{t}}-\pi
$$

where DO $\left[\mathrm{mg} \mathrm{L}^{-1}\right]$ is the measured dissolved oxygen concentration, $\mathrm{t}[\mathrm{h}]$ is time, $\mathrm{K}_{\mathrm{a}, 20}\left[\mathrm{~h}^{-1}\right]$ is the reaeration coefficient as established in the gas exchange experiment, $\mathrm{T}\left[{ }^{\circ} \mathrm{C}\right]$ is the water temperature, $\mathrm{I}_{\mathrm{t}}\left[\mathrm{mol}\right.$ photons $\left.\mathrm{m}^{-2} \mathrm{~s}^{-1}\right]$ is the surface photosynthetically active radiation, $\Phi_{\mathrm{K}}=$ 1.0241 is the coefficient for the Arrhenius type temperature dependence of the reaeration coefficient and $\mathrm{DO}_{\text {sat }}\left[\mathrm{mg} \mathrm{L}^{-1}\right]$ is the saturation concentration of dissolved oxygen estimated following Benson \& Krause (1980, 1984). Corrections for atmospheric pressure were made based on monthly mean air pressure data for each experimental site. The model uses a Michaelis Menten type relation to describe light saturation of primary production. We did not use the Arrhenius type temperature model suggested by Jeppesen et al. (2012) since we were particularly interested in investigating the temperature responses of the metabolic rates to the climate gradient. The parameter $\rho\left[\mathrm{mg} \mathrm{l}^{-1} \mathrm{~h}^{-1}\right]$ is interpreted as the maximum obtainable production rate, $\eta$ [mol photons $\mathrm{m}^{-2} \mathrm{~s}^{-1}$ ] is the light intensity at which the primary production reaches half its maximum (half saturation constant) and $\pi\left[\mathrm{mg}^{-1} \mathrm{~h}^{-1}\right]$ is the average rate of ecosystem respiration. GPP and ER per day are then calculated as follows:

$$
\begin{gathered}
\text { GPP }\left[\frac{\mathrm{mg}}{\mathrm{ld}}\right]=\rho \sum_{\mathrm{t}} \frac{\mathrm{I}_{\mathrm{t}}}{\eta+\mathrm{I}_{\mathrm{t}}} \Delta \mathrm{t} \\
\mathrm{ER}\left[\frac{\mathrm{mg}}{\mathrm{ld}}\right]=\pi \cdot 24
\end{gathered}
$$

where $\Delta \mathrm{t}[\mathrm{h}]$ is the time difference between two consecutive measurements.

For estimation of $\rho, \pi$ and $\eta$, we used the shuffled complex evolution optimisation algorithm (SCE) as implemented in the R package "hydromad" (Andrews \& Guillaume, 2014) to minimise the sum of the squared residuals. Starting values for all three parameters were found using a brute force grid search within the range 0.0001 to 5 applying the nls $2 \mathrm{R}$ package (Grothendieck, 2013). To assess the uncertainty of the estimated production and respiration rates, we used a bootstrap approach similar to that in Solomon et al. (2013) where the autocorrelation of lag 1 and the variance from the residuals of the original data are used to construct bootstrapped residuals with the same autocorrelation and variance as the original data. Those bootstrapped residuals are then added to the fitted values and the estimation, using the same starting values, is repeated for the so generated pseudo dDO replicates.

Estimates with standard errors larger than the estimate itself:

$$
\frac{\sqrt{\left(\frac{1}{500} \sum_{\mathrm{i}}^{500}\left(\mathrm{e}_{\mathrm{i}}-\mathrm{e}\right)^{2}\right)}}{\mathrm{e}}>1
$$

where $\mathrm{e}_{\mathrm{i}}$ are the bootstrapped estimates and $\mathrm{e}$ is the estimate from the raw data and estimates explaining less than $5 \%$ of the variability of the 24 -h dissolved oxygen curve were excluded 
from further analysis. Overall, 374 data points remained. For an overview of the distribution of data points per country, month and treatment, see S1 Table 2. To obtain day lengthcorrected GPP values, $\mathrm{GPP}_{\mathrm{dl}}\left[\mathrm{mg} \mathrm{L}^{-1} \mathrm{hd}^{-1}\right]$, GPP per day was divided by the average daylight period, LP $\left(\mathrm{hd}^{-1}\right)$, per month and country.

\section{S2.2 Estimation of light attenuation coefficient, $K_{d}$, mean available light and effective light period}

Global radiation was converted to PAR using the transformation given in Kirk (2010):

$$
\mathrm{PAR} \approx \mathrm{E} \cdot \gamma \cdot 0.45
$$

where PAR is given in $\mu \mathrm{mol} \mathrm{m} \mathrm{m}^{-2}$ and $\mathrm{E}$ in $\mathrm{W} \mathrm{m}^{-2} ; \gamma=4.6 \mu \mathrm{mol} \mathrm{\textrm {J } ^ { - 1 }}$ is a conversion factor based on the centre wavelength of $550 \mathrm{~nm}$ of the $400-700 \mathrm{~nm}$ waveband (McCree, 1981). For each light profile (d) and each concurrent light intensity measurement (i), the attenuation coefficient $\mathrm{K}_{\mathrm{di}}\left(\mathrm{m}^{-1}\right)$ was estimated based on the Beer-Lambert law:

$$
\mathrm{K}_{\mathrm{di}}=\frac{\ln \frac{\mathrm{I}_{\mathrm{i}}}{\mathrm{I}_{\mathrm{i}+1}}}{\mathrm{Z}_{\mathrm{i}+1}-\mathrm{z}_{\mathrm{i}}}
$$

, where $I_{i}$ and $I_{i+1}$ are PAR values at depth $z_{i}$ and $z_{i+1}$. Values with $I_{i+1}>I_{i}$ were removed. $\mathrm{K}_{\mathrm{d}}\left(\mathrm{m}^{-1}\right)$ was then taken as the mean of all $\mathrm{K}_{\mathrm{di}}$.

Mean available light $\left(\bar{I}, \mu \mathrm{mol}\right.$ photons $\left.\mathrm{m}^{-2} \mathrm{~s}^{-1}\right)$ was estimated as:

$$
\bar{I}=\frac{\mathrm{I}_{0}}{\mathrm{z} \cdot \mathrm{K}_{\mathrm{d}}}\left(1-\mathrm{e}^{-\mathrm{K}_{\mathrm{d}} \cdot \mathrm{z}}\right)
$$

, where $\mathrm{I}_{0}$ is the incident light just beneath the surface. If only incident light measurements were available, we corrected values for 10\% backscatter (Kirk, 2010; Staehr et al., 2010). Effective light period, LP ${ }_{\text {eff }}$, was calculated as described in Shatwell et al. (2012):

$$
\mathrm{LP}_{\mathrm{eff}}=\frac{\mathrm{z}_{1 \%}}{\mathrm{z}_{\mathrm{mix}}} \cdot \mathrm{LP}
$$

, where $\mathrm{z}_{1 \%}(\mathrm{~m})$ is the euphotic depth (depth at which $1 \%$ of surface light intensity is left), $\mathrm{z}_{\mathrm{mix}}$ is the mixing depth, coinciding in our fully mixed mesocosms with the water column height, and LP is the mean light period $\left(\mathrm{hd}^{-1}\right)$ per month and country. Values were limited to one since the experienced LP cannot be larger than the actual LP. If $\mathrm{z}_{1 \%}$ is lower than $\mathrm{z}_{\mathrm{mix}}$, the effective day length is shorter than the actual LP due to mixing. 
S2 Table 1: Overview of the used methods for water chemistry and chlorophyll a analysis.

\begin{tabular}{|c|c|c|c|}
\hline & TP & $\mathrm{TN}$ & Ch a \\
\hline Sweden & EN ISO 6878 & $\begin{array}{l}\text { EN ISO } 11905-1 \\
\text { Tecator AN 5202-SE with } \\
\text { a FIAstar } 5000 \text { system }\end{array}$ & $\begin{array}{l}\text { Spectrophotometrically at a wavelength of } 665 \\
\mathrm{~nm} \text { from acetone extraction. Material gained on } \\
\text { GF/F glass microfiber filters by filtering of the } \\
\text { water samples. }\end{array}$ \\
\hline Estonia & ISO 15681-2 & ISO 29441 & $\begin{array}{l}\text { Spectrophotometrically (Edler 1979) at a } \\
\text { wavelength of } 665 \mathrm{~nm} \text { from } 96 \% \text { ethanol } \\
\text { extracts. Material gained on GF/F glass } \\
\text { microfiber filters by filtering of the water } \\
\text { samples. }\end{array}$ \\
\hline Germany & EN ISO 6878 (DEV, D11) & EN 12260 (DEV, H 34) & $\begin{array}{l}\text { High-performance liquid chromatography } \\
\text { (Waters, USA) following Fietz \& Nicklish } \\
\text { (2004). }\end{array}$ \\
\hline $\begin{array}{l}\text { Czech } \\
\text { Republic }\end{array}$ & $\begin{array}{l}\text { TP was determined spectrophotometrically with a } \\
\text { molybdate method after perchloric acid digestion } \\
\text { according to Kopáček J. and Hejzlar J. (1993) } \\
\text { Semi-micro determination of total phosphorus in } \\
\text { fresh waters with perchloric acid digestion. Int. J. } \\
\text { Environ. Anal. Chem. 53, 173-183. }\end{array}$ & $\begin{array}{l}\text { EN } 12260 \text { (DEV, H 34) } \\
\text { Elementar vario TOC } \\
\text { cube analyser (Elementar } \\
\text { Analysensysteme GmbH, } \\
\text { Germany). }\end{array}$ & $\begin{array}{l}\text { Spectrophotometrically (Edler 1979) at a } \\
\text { wavelength of } 665 \mathrm{~nm} \text { from } 96 \% \text { ethanol } \\
\text { extracts. Material gained on glass-fibre filters } \\
\text { of } 0.4-\mu \text { m nominal pore size (GF-5, Macherey- } \\
\text { Nagel, Düren, Germany) by filtering of the } \\
\text { water samples. }\end{array}$ \\
\hline Turkey & ISO 6878:2004 & |ISO 29441:2010 & $\begin{array}{l}\text { Spectrophotometrically (Edler 1979) at a } \\
\text { wavelength of } 665 \mathrm{~nm} \text { from } 96 \% \text { ethanol } \\
\text { extracts. Material gained on GF/F glass } \\
\text { microfiber filters by filtering of the water } \\
\text { samples. }\end{array}$ \\
\hline Greece & ISO 6878:2004 & $\begin{array}{l}\text { ISO } 11905-1 \text { and then use } \\
\text { of a Shimanzu TOC- } \\
\text { VCS/CP analyzer, } \\
\text { equipped with TNM-1 TN } \\
\text { unit. }\end{array}$ & $\begin{array}{l}\text { Spectrophotometrically (Edler 1979) at a } \\
\text { wavelength of } 665 \mathrm{~nm} \text { from } 96 \% \text { ethanol } \\
\text { extracts. Material gained on GF/F glass } \\
\text { microfiber filters by filtering of the water } \\
\text { samples. }\end{array}$ \\
\hline
\end{tabular}

S2 Table 2: Overview of the used meteorological data and data providers. Global radiation was converted to PAR using the transformation given in Kirk (2010).

\begin{tabular}{|c|c|c|c|}
\hline $\begin{array}{l}\text { Country/ } \\
\text { Variable }\end{array}$ & Air temperature (AT) & Air pressure (AP) & $\begin{array}{l}\text { Photosynthetically active } \\
\text { radiation (PAR) or } \\
\text { Global radiation (GlobR) }\end{array}$ \\
\hline Sweden & $\begin{array}{l}\text { Laboratory Weather Station at Lake } \\
\text { Erken, Department of Ecology and } \\
\text { Genetics, Uppsala University. } \\
\text { Measured at Lake Erken. }\end{array}$ & $\begin{array}{l}\text { Swedish Meteorological and } \\
\text { Hydrological Institute. } \\
\text { Measured at Lake Erken. }\end{array}$ & $\begin{array}{l}\text { Swedish Meteorological and } \\
\text { Hydrological Institute. GlobR was } \\
\text { measured at Norrköping. }\end{array}$ \\
\hline Estonia & \multicolumn{3}{|c|}{$\begin{array}{l}\text { Centre of Limnology of the Estonian University of Life Sciences. } \\
\text { Measured at Lake Võrtsjärv. }\end{array}$} \\
\hline Germany & \multicolumn{3}{|c|}{$\begin{array}{l}\text { Leibniz-Institute of Freshwater Ecology and Inland Fisheries (IGB). } \\
\text { Measured at Lake Müggelsee. }\end{array}$} \\
\hline $\begin{array}{l}\text { Czech } \\
\text { Republic }\end{array}$ & $\begin{array}{l}\text { České Budĕjovice - Institute of } \\
\text { Hydrobiology. Measured at the the } \\
\text { Rímov Reservoir. }\end{array}$ & $\begin{array}{l}\text { České Budějovice }- \text { Czech } \\
\text { Hydrometeorological Institute. } \\
\text { Measured at the the Římov } \\
\text { Reservoir }\end{array}$ & $\begin{array}{l}\text { České Budějovice - Institute of } \\
\text { Hydrobiology. GlobR was } \\
\text { measured at the the Římov } \\
\text { Reservoir. }\end{array}$ \\
\hline Turkey & \multicolumn{3}{|c|}{ Turkish State Meteorology Service (Ankara) } \\
\hline Greece & \multicolumn{3}{|c|}{ Hellenic National Meteorological Service } \\
\hline
\end{tabular}




\section{S2 References}

Andrews F, Guillaume J (2014) hydromad: Hydrological Model Assessment and Development.

Benson BB, Krause DJ (1980) The concentration and isotopic fractionation of gases dissolved in freshwater in equilibrium with the atmosphere. 1. Oxygen. Limnology and Oceanography, 24, $662-671$.

Benson BB, Krause D (1984) The concentration and isotopic fractionation of oxygen dissolved in freshwater and seawater in equilibrium with the atmosphere. Limnology and Oceanography, 29, 620-632.

Edler L (1979) Recommendations for marine biological studies in the Baltic Sea. Phytoplankton and Chlorophyll. The Baltic Marine Biologists, 5, 1-38

Fietz S and Nicklish A (2004) An HPLC analysis of the summer phytoplankton assemblage in Lake Baikal. Freshwater Biology, 49, 332-345

Grothendieck G (2013) nls2: Non-linear regression with brute force.

Jeppesen E, Lauridsen TL, Davidson TA et al. (2012) Biomanipulation as a restoration tool to combat eutrophication: Recent advances and future challenges. Advances in Ecological Research, 47, 411-488.

Kirk JTO (2010) Light and Photosynthesis in Aquatic Ecosystems, 3rd edn. Cambridge University Press, $649 \mathrm{pp}$.

McCree KJ (1981) Photosynthetically active radiation. In: Physiological plant ecology. Lang OL, Novel P, Osmond B, Ziegler (ed). Vol. 12A, Encyclopedia of plant physiology (new series). Springer-Verlag. Berlin, Heidelberg, New York.

Shatwell T, Nicklisch A, Köhler J (2012) Temperature and photoperiod effects on phytoplankton growing under simulated mixed layer light fluctuations. Limnology and Oceanography, 57, 541-553.

Solomon CT, Bruesewitz DA, Richardson DC et al. (2013) Ecosystem respiration: Drivers of daily variability and background respiration in lakes around the globe. Limnology and Oceanography, 58, 849-866.

Staehr PA, Sand-Jensen K, Raun AL, Nilsson B, Kidmose J (2010) Drivers of metabolism and net heterotrophy in contrasting lakes. Limnology and Oceanography, 55, 817-830. 


\section{Appendix S3: Treatment differences in nutrients, light conditions, chlorophyll a and PVI and covariation with temperature}

Proxies to assess the effects of the differential monthly loading of phosphate and nitrogen are TP and TN levels. Average TP levels in deep mesotrophic and shallow eutrophic mesocosms were almost constant over the entire temperature gradient. Whereas TP concentrations in shallow mesotrophic mesocosms increased with temperature (on average $10.2 \mu \mathrm{g} \mathrm{L}{ }^{-1}$ over 7 to $29^{\circ} \mathrm{C}$ ), and decreased in the deep eutrophic mesocosms (on average $30.6 \mu \mathrm{g} \mathrm{TP} \mathrm{L}{ }^{-1}$ over 7 to $29^{\circ} \mathrm{C}$ ). Despite these dynamics, all treatments exhibited significantly different TP levels over the entire temperature gradient (Figure 2, S3 Table 1).

The average TN concentration showed only slight changes over the temperature gradient for all treatments except in the deep eutrophic mesocosms where they decreased with, on average, $1.5 \mathrm{mg}$ $\mathrm{TN} \mathrm{L}^{-1}$ along the whole temperature gradient from 7 to $29^{\circ} \mathrm{C}$. Due to these differences in dynamics, TN levels between deep eutrophic and both mesotrophic treatments were no longer significantly different for temperatures above $25^{\circ} \mathrm{C}$ (SL) and $26^{\circ} \mathrm{C}$ (DL) (Figure 2, S3 Table 1).

Average light attenuation $\left(\mathrm{K}_{\mathrm{d}}\right)$ was significantly higher in the eutrophic than in the mesotrophic mesocosms. For the contrast between shallow mesotrophic and deep eutrophic mesocosms, this is, however, only true for temperatures above $10^{\circ} \mathrm{C}$. Generally, average light attenuation showed only small changes over the temperature gradient, with the exception of the shallow eutrophic mesocosms where attenuation decreased with increasing temperatures $\left(1.1 \mathrm{~m}^{-1}\right.$ from 7 to $\left.29{ }^{\circ} \mathrm{C}\right)(\mathrm{S} 3$ Figure 1a, S3 Table 1).

Average mean available light (MAL) was highest in the shallow mesotrophic mesocosms (adjusted mean: $111 \mu \mathrm{mol}$ photons $\mathrm{m}^{-2} \mathrm{~s}^{-1}$ ) along the entire temperature gradient, followed by the shallow eutrophic, deep mesotrophic mesocosms and, finally, the deep eutrophic mesocosms (adjusted mean: $41 \mu \mathrm{mol}$ photons $\mathrm{m}^{-2} \mathrm{~s}^{-1}$ ). MAL increased strongly with temperature for the shallow mesocosms and slightly less for the deep mesocosms, leading to more pronounced differences between treatments with increasing temperature. MAL differed significantly for all pairwise treatment comparisons over the temperature gradient. However, for the deep mesotrophic and shallow eutrophic mesocosms, this was only true for temperatures higher than $9^{\circ} \mathrm{C}$ (S3 Figure $1 \mathrm{~b}$, S3 Table 1).

The average effective light period $\left(\mathrm{LP}_{\text {eff }}\right)$ was longest in the shallow mesotrophic mesocosms (adjusted mean: $13.6 \mathrm{~h} \mathrm{~d}^{-1}$ ); however, $\mathrm{LP}_{\text {eff }}$ in the shallow eutrophic and deep mesotrophic mesocosms was only slightly shorter. $\mathrm{LP}_{\text {eff }}$ in the deep mesotrophic mesocosms was only significantly shorter than in the shallow mesocosms for temperatures above $15{ }^{\circ} \mathrm{C}$ (SL) and $21{ }^{\circ} \mathrm{C}$ $(\mathrm{SH})$. Along the entire temperature gradient, the deep eutrophic mesocosms had a significantly shorter $\mathrm{LP}_{\text {eff }}$ (adjusted mean: 11.47h LP $\mathrm{Lff}_{\mathrm{h} \mathrm{d}} \mathrm{h}^{-1}$ ) (S3 Figure 1c, S3 Table 1).

The coinciding increase in temperature with daylight hours was more pronounced in the northern and mid-European than in the southern European countries (S3 Figure 1d).

Average chlorophyll a levels were significantly higher under eutrophic (adjusted mean: 25.35 (DH) and $15.57\left(\mathrm{SH} \mu \mathrm{g} \mathrm{L}^{-1}\right)$ than mesotrophic (6.54 (DL), $7.25(\mathrm{SL}) \mu \mathrm{g} \mathrm{L}^{-1}$ ) conditions. In the eutrophic mesocosms, chlorophyll a decreased significantly along the temperature gradient but remained almost constant in the mesotrophic mesocosms. Despite these dynamics, average chlorophyll a levels remained significantly higher in the eutrophic than in the mesotrophic mesocosms along the entire temperature gradient (S3 Figure 2a, S3 Table 1).

PVI levels showed a trend opposite to that of chlorophyll a and were highest in the shallow mesotrophic mesocosm (adjusted mean: 8.09\%), followed by the shallow eutrophic and deep mesotrophic mesocosms with comparable values (adjusted mean: 3.51\% (SH) and $3.89 \%$ (DL)) and, finally, the deep eutrophic mesocosms $(0.71 \%)$. PVI decreased strongly with increasing temperatures in all treatments but the deep, eutrophic mesocosms where similar low average values 
were observed along the entire temperature gradient (S3 Figure 2b, S3 Table 1).
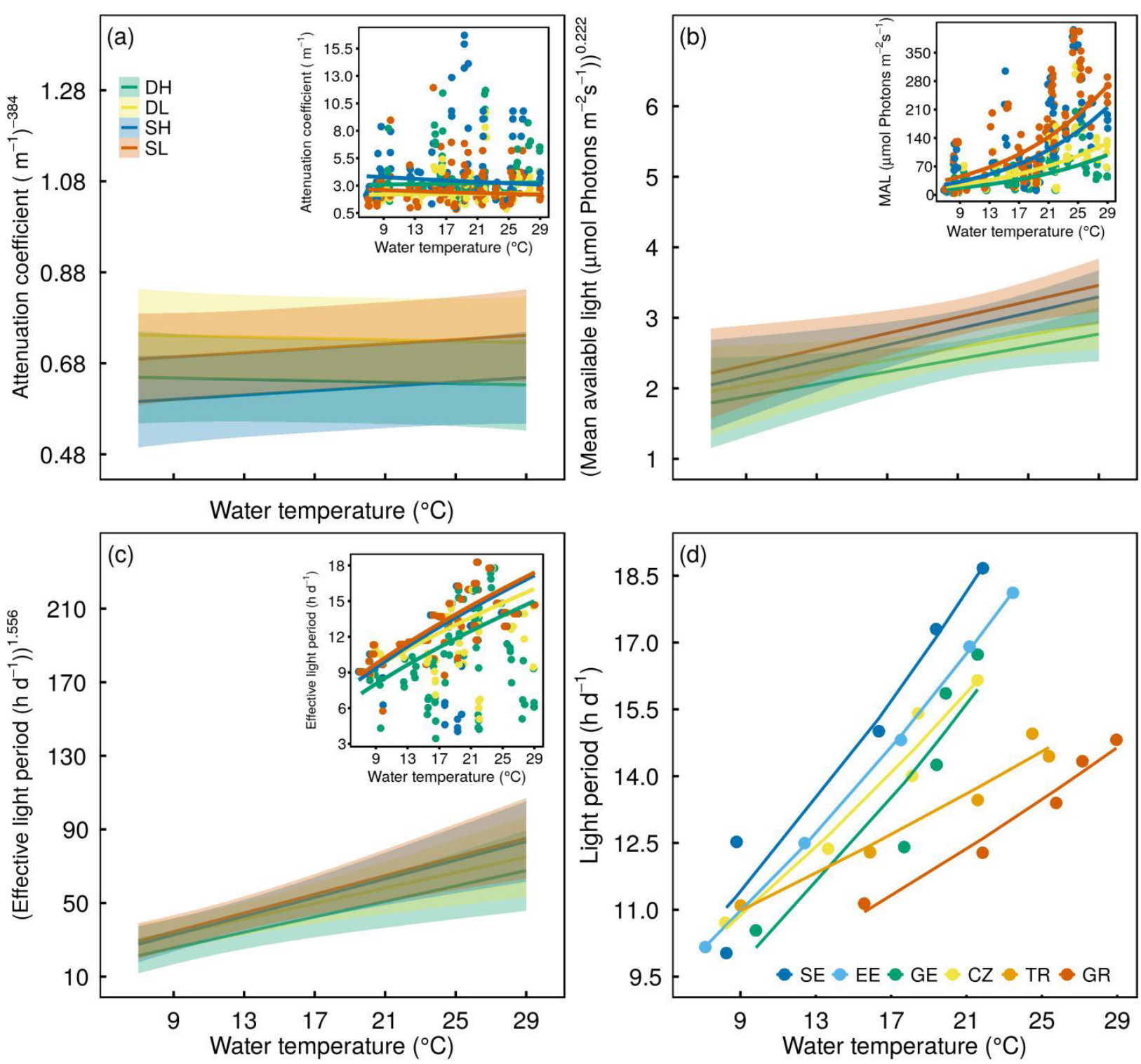

S3 Figure 1: Co-variation of light conditions with water temperature. Treatment-wise covariation of a) attenuation coefficient $\left(\mathrm{K}_{\mathrm{d}}\right), \mathrm{b}$ ) mean availability light (MAL) and c) effective light period $\left(\mathrm{LP}_{\text {eff }}\right)$. Main images in $\left.\mathrm{a}\right), \mathrm{b}$ ) and c) show treatment-wise least square means with 95\% confidence intervals at the scale of the transformed variable. The insets depict the same variable at original scale with treatment-wise average values as estimated by mixed effects regression (S3 Table 1). Lines in d) depict country-wise average monthly daylight hours per day estimated by linear regression. $\mathrm{DH}=$ deep high nutrient, $\mathrm{SH}=$ shallow high nutrient, $\mathrm{DL}=$ deep low nutrient and SL = shallow low nutrient treatment. 

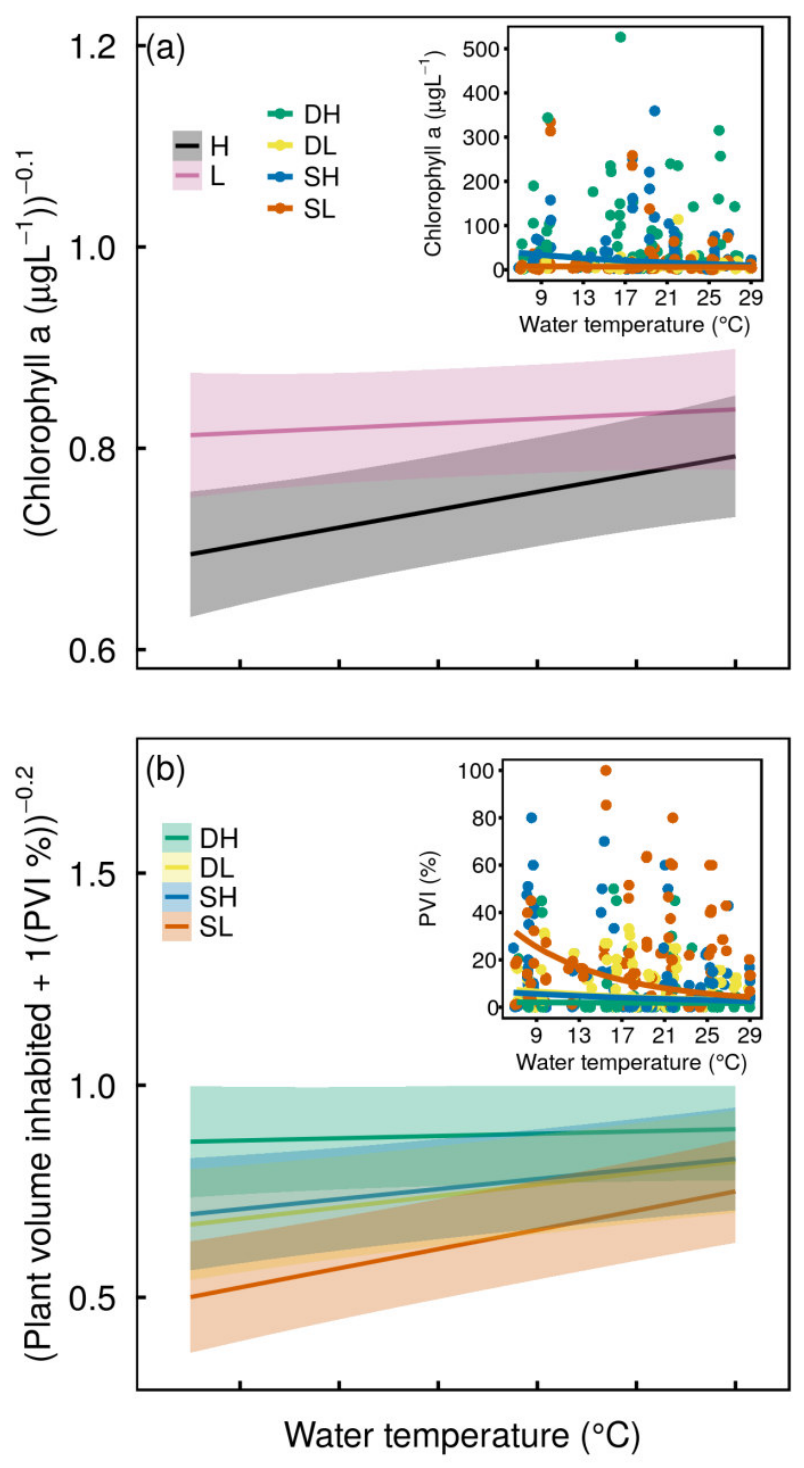

S3 Figure 2: Co-variation of primary producers with water temperature (S3 Table 1). Main image shows least-square means of significant factors with $95 \%$ confidence intervals on the scale of the transformed variable. The inserts depict values at original scale with average values transformed to original scale.

Treatment-wise co-variation of a) phytoplankton measured as chlorophyll $\mathrm{a}$ and $\mathrm{b}$ ) macrophytes measured as plant volume inhabited (PVI). $\mathrm{DH}=$ deep high nutrient, $\mathrm{SH}=$ shallow high nutrient, $\mathrm{DL}=$ deep low nutrient and $\mathrm{SL}=$ shallow low nutrient. 
S3 Table 1: Mixed effects regression for potential covariates. The influences of water temperature (T), depth (D) and nutrients (N), as well as their interactions, were tested on: total phosphorus (TP), total nitrogen (TN), attenuation coefficient $\left(\mathrm{K}_{\mathrm{d}}\right)$, mean available light (MAL), effective light period $\left(\mathrm{LP}_{\text {eff }}\right)$, chlorophyll a $\left(\mathrm{Chl}\right.$ a) and plant volume inhabited (PVI). Only p-values $<=0.05$ are reported. The first $\mathrm{R}^{2}$ value refers to the marginal $\mathrm{R}^{2}$

(variance explained by fixed factors) and the second to the conditional $\mathrm{R}^{2}$ (variance explained by fixed and random factors). The next four columns show treatment-wise adjusted means and average differences over the water temperature gradient, i.e. the difference between average values at $7{ }^{\circ} \mathrm{C}$ and $29^{\circ} \mathrm{C}$. The last 6 columns show the results of pairwise comparison of least-square means over the temperature gradient in one degree intervals. Temperatures in brackets indicate temperatures from where on significant treatment differences were found at a 0.05 significance level.

\begin{tabular}{|c|c|c|c|c|c|c|c|c|c|c|c|c|c|c|c|c|c|c|}
\hline & \multirow[b]{2}{*}{$\mathbf{T}$} & \multirow[b]{2}{*}{ D } & \multirow[b]{2}{*}{$\mathbf{N}$} & \multirow[b]{2}{*}{$T \times D$} & \multirow[b]{2}{*}{$\mathbf{T} \times \mathbf{N}$} & \multirow[b]{2}{*}{ D $\times N$} & \multirow[b]{2}{*}{$T \times D \times N$} & \multirow[b]{2}{*}{$\mathbf{R}^{2}$} & \multicolumn{4}{|c|}{$\begin{array}{c}\begin{array}{c}\text { Adjusted mean and gradient } \\
\text { difference }\end{array} \\
\end{array}$} & \multicolumn{6}{|c|}{ Contrasts over temperature gradient } \\
\hline & & & & & & & & & SH & DH & SL & DL & SL-SH & DL-DH & SL - DH & DL - SH & $\mathbf{S L}-\mathbf{D L}$ & DH - SH \\
\hline $\mathrm{TP}\left[\mu \mathrm{gL}^{-1}\right]$ & & & $<0.01$ & 0.03 & 0.02 & & & $0.39,0.88$ & $\begin{array}{l}68.92, \\
0.43 \\
\end{array}$ & $\begin{array}{r}71.02, \\
-30.63 \\
\end{array}$ & $\begin{array}{l}26.32, \\
10.24 \\
\end{array}$ & $\begin{array}{c}21.90, \\
0.30 \\
\end{array}$ & $\mathrm{p}=<0.05$ & $\mathrm{p}=<0.05$ & $\mathrm{p}=<0.05$ & $\mathrm{p}=<0.05$ & $\mathrm{p}=<0.05$ & $\mathrm{p}=<0.05$ \\
\hline $\mathbf{T N}\left[\mathrm{mgL}^{-1}\right]$ & & & $<0.01$ & 0.03 & $<0.01$ & & $<0.01$ & $0.31,0.88$ & $\begin{array}{l}1.37 \\
-0.29\end{array}$ & $\begin{array}{l}1.43 \\
-1.54\end{array}$ & $\begin{array}{r}0.79 \\
0.11\end{array}$ & $\begin{array}{c}0.71 \\
0.28\end{array}$ & $\mathrm{p}=<0.05$ & $\begin{array}{c}\mathrm{p}=<0.05 \\
\left(<26^{\circ} \mathrm{C}\right)\end{array}$ & $\begin{array}{l}\mathrm{p}=<0.05 \\
\left(<25^{\circ} \mathrm{C}\right)\end{array}$ & $\mathrm{p}=<0.05$ & n.s. & $\begin{array}{c}\mathrm{p}=<0.05 \\
\left(<13{ }^{\circ} \mathrm{C},\right. \\
\left.>24{ }^{\circ} \mathrm{C}\right)\end{array}$ \\
\hline $\mathbf{K}_{\mathrm{d}}\left[\mathbf{m}^{-1}\right]$ & & & $<0.01$ & 0.01 & & & & $0.12,0.76$ & $\begin{array}{l}3.45 \\
-1.12 \\
\end{array}$ & $\begin{array}{c}3.17 \\
0.03 \\
\end{array}$ & $\begin{array}{l}2.38 \\
-0.18 \\
\end{array}$ & $\begin{array}{l}2.25 \\
0.22 \\
\end{array}$ & $\mathrm{p}=<0.05$ & $\mathrm{p}=<0.05$ & $\begin{array}{l}\mathrm{p}=<0.05 \\
\left(>10^{\circ} \mathrm{C}\right)\end{array}$ & $\mathrm{p}=<0.05$ & $\begin{array}{l}\mathrm{p}=<0.05 \\
\left(<14^{\circ} \mathrm{C}\right)\end{array}$ & $\begin{array}{l}\mathrm{p}=<0.05 \\
\left(<14^{\circ} \mathrm{C}\right)\end{array}$ \\
\hline \begin{tabular}{|l|} 
MAL [ $\mu \mathrm{mol}$ \\
Photons $\mathbf{~ m}^{-2} \mathbf{s}^{-1}$ ] \\
\end{tabular} & 0.02 & $<0.01$ & $<0.01$ & $<0.01$ & & & & $0.43,0.94$ & $\begin{array}{c}85.90 \\
177.41 \\
\end{array}$ & $\begin{array}{l}41.29 \\
76.95 \\
\end{array}$ & $\begin{array}{l}110.56 \\
213.53\end{array}$ & $\begin{array}{c}57.30 \\
100.48 \\
\end{array}$ & $\mathrm{p}=<0.05$ & $\mathrm{p}=<0.05$ & $\mathrm{p}=<0.05$ & $\begin{array}{c}\mathrm{p}=<0.05 \\
\left(>9^{\circ} \mathrm{C}\right)\end{array}$ & $\mathrm{p}=<0.05$ & $\mathrm{p}=<0.05$ \\
\hline $\mathbf{L P} \mathbf{P}_{\text {eff }}\left[h^{-1}\right]$ & $<0.01$ & $<0.01$ & $<0.01$ & 0.01 & & 0.03 & & $0.45,0.89$ & $\begin{array}{l}13.23 \\
7.74 \\
\end{array}$ & $\begin{array}{c}11.47 \\
6.89 \\
\end{array}$ & $\begin{array}{c}13.60 \\
8.20 \\
\end{array}$ & $\begin{array}{l}12.76 \\
7.07 \\
\end{array}$ & n.s. & $\mathrm{p}=<0.05$ & $\mathrm{p}=<0.05$ & \begin{tabular}{|l|}
$\mathrm{p}=<0.05$ \\
$\left(>21{ }^{\circ} \mathrm{C}\right)$
\end{tabular} & $\begin{array}{l}\mathrm{p}=<0.05 \\
\left(>15^{\circ} \mathrm{C}\right) \\
\end{array}$ & $\mathrm{p}=<0.05$ \\
\hline Chl a $\left[\mu g^{-1}\right]$ & & & $<0.01$ & & 0.02 & & & $0.19,0.76$ & $\begin{array}{r}15.57 \\
-20.21 \\
\end{array}$ & $\begin{array}{r}25.35 \\
-32.57 \\
\end{array}$ & $\begin{array}{l}7.25 \\
-2.33 \\
\end{array}$ & $\begin{array}{l}6.54 \\
-2.03 \\
\end{array}$ & \multicolumn{6}{|c|}{$\mathrm{p}=<0.05$ for $\mathrm{H}-\mathrm{L}$ (depth was not in the final model) } \\
\hline PVI [\%] & 0.01 & $<0.01$ & $<0.01$ & 0.02 & 0.01 & & & $0.22,0.86$ & $\begin{array}{l}3.51, \\
-4.98 \\
\end{array}$ & $\begin{array}{l}0.71, \\
-0.04 \\
\end{array}$ & $\begin{array}{l}8.09 \\
-16.86 \\
\end{array}$ & $\begin{array}{l}3.89 \\
-5.83 \\
\end{array}$ & $\mathrm{p}=<0.05$ & $\mathrm{p}=<0.05$ & $\mathrm{p}=<0.05$ & n.s. & $\mathrm{p}=<0.05$ & $\mathrm{p}=<0.05$ \\
\hline
\end{tabular}




\section{Appendix S4: Country-wise temperature and treatment effects of metabolism}

To obtain an idea of the variability between countries for absolute metabolism at $15{ }^{\circ} \mathrm{C}$ and the activation energy, we included the factor country and the interaction between country and inversescaled temperature in the fixed effects of the model (6). The country-wise analysis for GPP, ER and ER/GPP ratio was based on the following mixed effect model:

$$
\left.\ln (\text { Parameter })=\operatorname{invT}+\mathrm{D}+\mathrm{N}+\text { Country }+ \text { invT x Country+(invT } \mid \text { Country: } \operatorname{Id}_{\text {meso }}\right)+(1 \mid \text { Country:Month })
$$

, where invT is the inverse-scaled water temperature, $\mathrm{D}$ and $\mathrm{N}$ are the treatment factors for depth and nutrient level, respectively, Country stands for the factor country, $\operatorname{Id}_{\text {meso }}$ uniquely identifies each mesocosm and Month stands for the factor month. An intercept and slope model for the random factor $\mathrm{Id}_{\text {meso }}$ nested in country and an intercept model for the random factor month nested in the country were considered. Model selection, validation and fit are assessed as described in the method part of the main text. Following the hypothesis that warm adaptation leads to lower temperature sensitivity than cold adaptation (Atkin, 2003), we would expect a systematic decrease in activation energy with increasing average country temperature and, possibly, a decrease in average absolute metabolic rate at the reference temperature. We tested for systematic increases or decreases in country-wise activation energies and average absolute metabolic rates using a Helmert contrast with the factor country following the same order as the country-wise average air temperature (Table 1) over the experimental period (SE, EE, CZ, GE, TR, GR). The results are summarised in S4 Table 1. In addition, Spearman correlations between average temperature of the countries and estimated country-wise absolute metabolic rates and activation energies were calculated (S4 Table 2).

Temperature sensitivity was highly variable between countries, yet we found no indication for a systematic change with average temperature in either temperature sensitivity or absolute metabolic rate at $15{ }^{\circ} \mathrm{C}$. Thus, we have good justification for our main model (equation 6), were we test for one generic temperature sensitivity as suggested by the MTE and account for random variation around this temperature sensitivity with the help of random effects. The design of this experiment, however, does not allow a thorough investigation of the effects of temperature acclimation and adaptation on the temperature sensitivity of metabolic rates, due to the lack of replicates per average temperature and the limited amount of experimental sites. 
S4 Table 1: Statistical results for models with country as fixed effect. The following abbreviations are used: Int $=$ Intercept at $15{ }^{\circ} \mathrm{C}(\mathrm{Tc})$, invT $=$ Inverted and scaled water temperature $\mathrm{T}$ in Kelvin $(\mathrm{k}$ $\left.{ }^{1} \mathrm{~T}^{-1}-\mathrm{k}^{-1} \mathrm{Tc}^{-1}\right), \mathrm{D}=$ Depth, $\mathrm{N}=$ Nutrient, $\mathrm{SE}=$ Sweden, $\mathrm{EE}=$ Estonia, $\mathrm{CZ}=$ Czech Republic, GE = Germany, TR = Turkey, GR = Greece. Systematic differences between countries were tested using a Helmert contrast, for instance Country_SE_EE:CZ is the difference between average metabolic rates at $15^{\circ} \mathrm{C}$ of SE and $\mathrm{EE}$ against the average rate in $\mathrm{CZ}$, and invT $\mathrm{x}$ Country_SE_EE:CZ is the difference in average activation energy of SE and $\mathrm{EE}$ against the difference in average activation energy of CZ.

\begin{tabular}{|c|c|c|c|c|c|c|}
\hline Response & Predictor & Estimate & Std. error & T-value & P-value & $\mathbf{R}^{2}$ \\
\hline \multirow{14}{*}{$\ln (\mathrm{GPP})$} & Int & 11.40 & 0.07 & 160.69 & $<0.01$ & \multirow{14}{*}{$0.65,0.80$} \\
\hline & invT & -0.78 & 0.07 & -6.69 & $<0.01$ & \\
\hline & D & 0.57 & 0.03 & -9.88 & $<0.01$ & \\
\hline & $\mathrm{N}$ & -0.55 & 0.03 & 10.45 & $<0.01$ & \\
\hline & Country_SE:EE & -0.08 & 0.10 & -2.10 & 0.04 & \\
\hline & Country_SE_EE:CZ & 0.04 & 0.06 & 3.52 & $<0.01$ & \\
\hline & Country_SE_EE_CZ:GE & 0.04 & 0.04 & 1.22 & 0.23 & \\
\hline & Country_SE_EE_CZ_GE:TR & 0.02 & 0.03 & 1.91 & 0.07 & \\
\hline & Country_SE_EE_CZ_GE_TR:GR & 0.09 & 0.05 & 2.31 & 0.03 & \\
\hline & invT x Country_SE:EE & -0.40 & 0.12 & -2.08 & 0.05 & \\
\hline & invT x Country_SE_EE:CZ & 0.50 & 0.08 & 4.17 & $<0.01$ & \\
\hline & invT x Country _SE_EE_CZ:GE & 0.03 & 0.06 & 0.34 & 0.74 & \\
\hline & invT x Country_SE_EE_CZ_GE:TR & 0.13 & 0.03 & 2.46 & 0.02 & \\
\hline & invT x Country_SE_EE_CZ_GE_TR:GR & 0.06 & 0.03 & 1.04 & 0.31 & \\
\hline \multirow{14}{*}{$\ln (\mathrm{ER})$} & Int & 11.43 & 0.09 & 127.04 & $<0.01$ & \multirow{14}{*}{$0.64,0.86$} \\
\hline & invT & -0.94 & 0.09 & -6.86 & $<0.01$ & \\
\hline & $\mathrm{D}$ & -0.58 & 0.03 & -9.40 & $<0.01$ & \\
\hline & $\mathrm{N}$ & 0.48 & 0.03 & 7.93 & $<0.01$ & \\
\hline & Country_SE:EE & -0.24 & 0.13 & -4.15 & $<0.01$ & \\
\hline & Country_SE_EE:CZ & 0.00 & 0.08 & 1.87 & 0.07 & \\
\hline & Country_SE_EE_CZ:GE & 0.05 & 0.06 & 1.91 & 0.07 & \\
\hline & Country_SE_EE_CZ_GE:TR & 0.08 & 0.04 & 3.18 & $<0.01$ & \\
\hline & Country_SE_EE_CZ_GE_TR:GR & 0.20 & 0.05 & 5.04 & $<0.01$ & \\
\hline & invT x Country_SE:EE & -0.97 & 0.14 & -4.25 & $<0.01$ & \\
\hline & invT x Country_SE_EE:CZ & 0.44 & 0.09 & 3.13 & 0.01 & \\
\hline & invT x Country_SE_EE_CZ:GE & 0.19 & 0.07 & 1.73 & 0.10 & \\
\hline & invT x Country_SE_EE_CZ_GE:TR & 0.16 & 0.04 & 2.53 & 0.02 & \\
\hline & invT x Country_SE_EE_CZ_GE_TR:GR & 0.22 & 0.04 & 3.53 & $<0.01$ & \\
\hline \multirow{8}{*}{$\ln \left(\mathrm{GPP}_{\mathrm{dl}}\right)$} & Int & 8.74 & 0.07 & 116.72 & $<0.01$ & \multirow{8}{*}{$0.61,0.78$} \\
\hline & invT & -0.43 & 0.08 & -3.47 & $<0.01$ & \\
\hline & $\mathrm{D}$ & -0.54 & 0.03 & -9.71 & $<0.01$ & \\
\hline & $\mathrm{N}$ & 0.58 & 0.03 & 10.43 & $<0.01$ & \\
\hline & Country_SE:EE & -0.04 & 0.11 & -1.72 & 0.10 & \\
\hline & Country_SE_EE:CZ & 0.06 & 0.06 & 3.67 & $<0.01$ & \\
\hline & Country_SE_EE_CZ:GE & 0.06 & 0.05 & 1.69 & 0.10 & \\
\hline & Country_SE_EE_CZ_GE:TR & 0.05 & 0.04 & 2.31 & 0.03 & \\
\hline
\end{tabular}




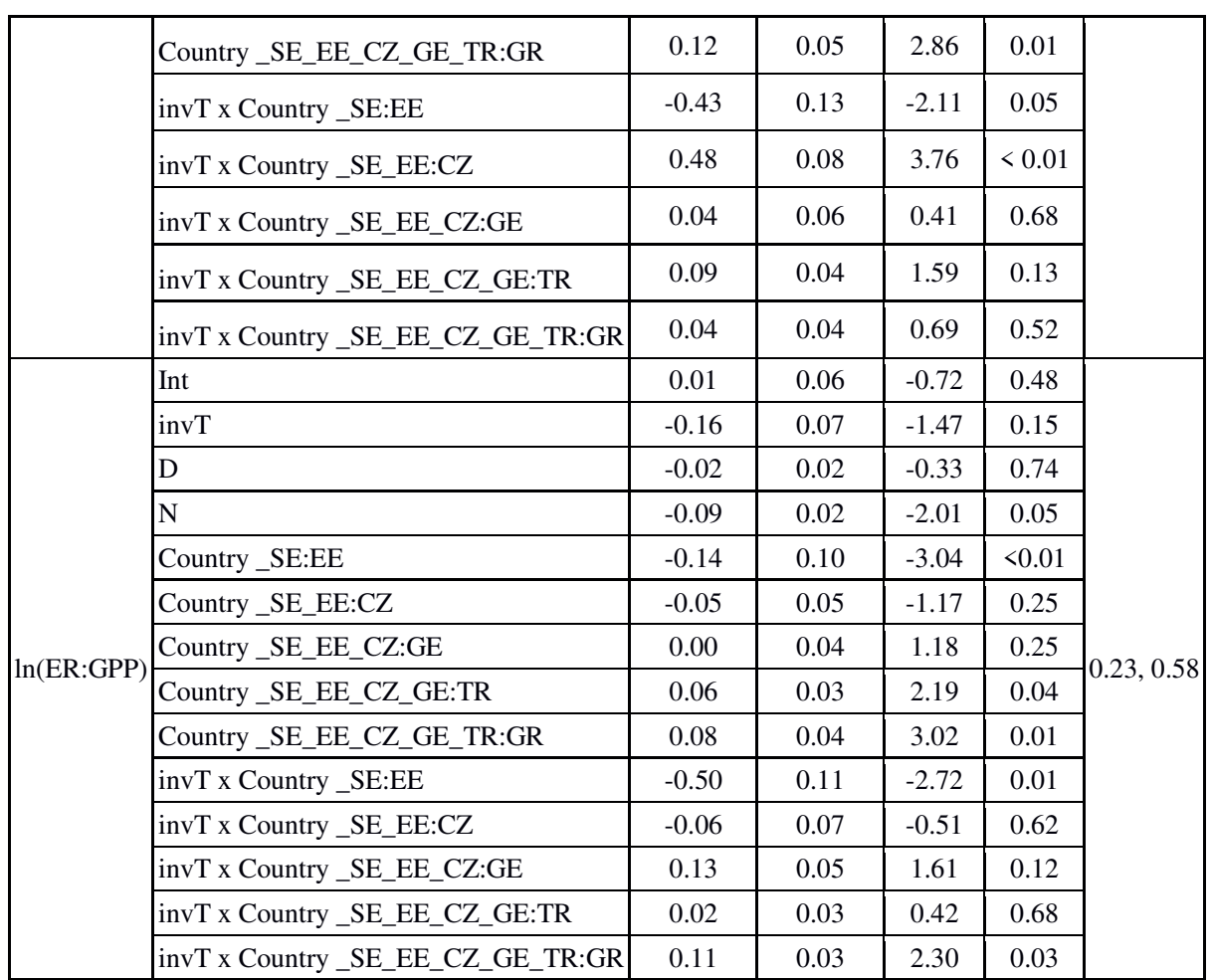

S4 Table 2: Table of country-wise activation energies and metabolic rates at $15{ }^{\circ} \mathrm{C}$ plus/minus standard error. In addition, Spearman correlation coefficients (r) are calculated between countrywise average water temperature and activation energies and metabolic rates at $15{ }^{\circ} \mathrm{C}$.

\begin{tabular}{|c|c|c|c|c|c|}
\hline & & GPP & $\mathbf{G P P}_{\mathrm{dl}}$ & ER & ER/GPP \\
\hline \multirow[t]{7}{*}{ Slope [eV] } & SE & $-0.69 \pm 0.15$ & $-0.41 \pm 0.16$ & $-0.62 \pm 0.18$ & $0.08 \pm 0.14$ \\
\hline & EE & $-1.19 \pm 0.18$ & $-0.95 \pm 0.19$ & $-1.82 \pm 0.22$ & $-0.54 \pm 0.18$ \\
\hline & $\mathrm{CZ}$ & $0.00 \pm 0.19$ & $0.23 \pm 0.21$ & $-0.39 \pm 0.22$ & $-0.34 \pm 0.18$ \\
\hline & GE & $-0.55 \pm 0.21$ & $-0.28 \pm 0.22$ & $-0.48 \pm 0.24$ & $0.07 \pm 0.13$ \\
\hline & TR & $-0.20 \pm 0.14$ & $-0.07 \pm 0.15$ & $-0.33 \pm 0.17$ & $-0.12 \pm 0.13$ \\
\hline & GR & $-0.31 \pm 0.19$ & $-0.15 \pm 0.20$ & $0.11 \pm 0.22$ & $0.26 \pm 0.17$ \\
\hline & $\begin{array}{l}\text { Correlation coefficient with } \\
\text { mean air temperature }\end{array}$ & $\begin{array}{l}\mathrm{r}=0.54 \\
\mathrm{p}=0.30\end{array}$ & $\begin{array}{l}r=0.54 \\
p=0.30\end{array}$ & $\begin{array}{l}\mathrm{r}=0.88 \\
\mathrm{p}=0.3\end{array}$ & $\begin{array}{l}r=0.37 \\
p=0.50\end{array}$ \\
\hline \multirow{7}{*}{$\begin{array}{l}\text { Intercept at } 15^{\circ} \mathrm{C} \\
\text { (average of treatments) }\end{array}$} & SE & $10.94 \pm 0.13$ & $8.27 \pm 0.14$ & $11.02 \pm 0.17$ & 0.08 \\
\hline & EE & $10.52 \pm 0.16$ & $7.90 \pm 0.17$ & $9.92 \pm 0.21$ & -0.52 \\
\hline & $\mathrm{CZ}$ & $11.33 \pm 0.14$ & $8.74 \pm 0.15$ & $10.89 \pm 0.19$ & -0.41 \\
\hline & GE & $11.14 \pm 0.16$ & $8.61 \pm 0.17$ & $11.04 \pm 0.20$ & -0.09 \\
\hline & TR & $11.30 \pm 0.15$ & $8.79 \pm 0.16$ & $11.40 \pm 0.20$ & 0.10 \\
\hline & GR & $11.67 \pm 0.27$ & $9.29 \pm 0.28$ & $12.48 \pm 0.31$ & 0.56 \\
\hline & $\begin{array}{l}\text { Correlation coefficient with } \\
\text { mean air temperature }\end{array}$ & $\begin{array}{l}r=0.77 \\
p=0.10\end{array}$ & $\begin{array}{l}\mathrm{r}=0.89 \\
\mathrm{p}=0.03\end{array}$ & $\begin{array}{l}r=0.83 \\
p=0.06\end{array}$ & $\begin{array}{l}r=0.66 \\
p=0.18\end{array}$ \\
\hline
\end{tabular}




\section{Appendix S5: Details of the final random effect models}

For all tested metabolic rates we started with the model given in equation (6). The "step" function of the package "ImerTest" (Kuznetsova et al., 2014) was than used for model selection. This function evaluates the significance of both fixed and random effects based on likelihood ratio tests (LRT), i.e. all terms which remain in the final model are significant based on a LRT (see S5 Table 1). Confidence intervals for the random and fixed effects were established using likelihood profiles as implemented in the function "confint.merMod" of the "lme4" package (Bates et al., 2014) (see S5 Table 2).

S5 Table 1: P-values of the random effects of the final metabolic rate models as estimated by likelihood ratio tests ("step" function of the package "ImerTest" (Kuznetsova et al., 2014)). '-' indicates that this random term was not significant and thus not part of the final model.

\begin{tabular}{|l|c|c|c|c|}
\hline Response & InvT| Country & InvT| Country: Mesocosm_Id & 1| Country: Mesocosm_Id & 1|Country:Month \\
\hline GPP & 0.00 & - & 0.00 & 0.00 \\
\hline GPP $_{\text {dl }}$ & 0.02 & - & 0.00 & 0.00 \\
\hline ER & 0.00 & 0.00 & - & 0.00 \\
\hline ER/GPP & 0.04 & 0.00 & - & 0.00 \\
\hline
\end{tabular}

S5 Table 2: Confidence intervals based on likelihood profiles ("confint.merMod" of the "Ime4" package (Bates et al., 2014)).

\begin{tabular}{|l|c|c|c|c|c|c|c|c|}
\hline & \multicolumn{2}{|c|}{ GPP } & \multicolumn{2}{c|}{ GPP $_{\mathrm{dl}}$} & \multicolumn{2}{c|}{ ER } & \multicolumn{2}{c|}{ ER/GPP } \\
\hline & $2.5 \%$ & $97.5 \%$ & $2.5 \%$ & $97.5 \%$ & $2.5 \%$ & $97.5 \%$ & $2.5 \%$ & $97.5 \%$ \\
\hline Sd Country:Id $\left(\epsilon_{\mathrm{R}}^{\mathrm{c}, \mathrm{m}}\right)$ & 0.12 & 0.24 & 0.12 & 0.24 & 0.3 & 0.54 & 0.2 & 0.35 \\
\hline Sd InvT: $\mid$ Country::Id $\left(\epsilon_{\mathrm{E}}^{\mathrm{c}, \mathrm{m}}\right)$ & - & - & - & - & 0.12 & 0.29 & 0.16 & 0.31 \\
\hline Sd Country:Month $\left(\epsilon_{\mathrm{R}}^{\mathrm{c}, \mathrm{S}}\right)$ & 0.17 & 0.35 & 0.18 & 0.38 & 0.18 & 0.42 & 0.12 & 0.27 \\
\hline Sd Country $\left(\epsilon_{\mathrm{R}}^{\mathrm{c}}\right)$ & 0.1 & 0.63 & 0.17 & 0.83 & 0.37 & 1.44 & 0.12 & 0.64 \\
\hline Sd InvT|Country $\left(\epsilon_{\mathrm{E}}^{\mathrm{c}}\right)$ & 0.15 & 0.72 & 0.09 & 0.66 & 0.25 & 1.07 & 0.04 & 0.44 \\
\hline Random error $\left(\epsilon_{\mathrm{c},(\mathrm{s}, \mathrm{m}), \mathrm{i}}\right.$ & 0.33 & 0.39 & 0.33 & 0.39 & 0.33 & 0.4 & 0.31 & 0.37 \\
\hline Intercept & 10.82 & 11.44 & 8.21 & 9.01 & 10.39 & 11.75 & -0.41 & 0.21 \\
\hline InvT & -0.9 & -0.2 & -0.65 & 0.01 & -1.14 & -0.11 & -0.36 & 0.08 \\
\hline Depth & 0.44 & 0.66 & 0.43 & 0.65 & 0.45 & 0.7 & -0.08 & 0.1 \\
\hline Nutrient & -0.68 & -0.47 & -0.69 & -0.47 & -0.6 & -0.36 & 0 & 0.17 \\
\hline
\end{tabular}

\section{S5 References}

Bates D, Maechler M, Bolker B, Walker S (2014)._lme4: Linear mixed-effects models using Eigen and S4_. R package version 1.1-7, <URL:http://CRAN.R-project.org/package=lme4>.

Kuznetsova A, Brockhoff PB and Christensen RHB (2014). lmerTest: Tests in Linear Mixed Effects Models. R package version 2.0-20. https://CRAN.R-project.org/package=lmerTest 


\section{Appendix S6: Evidence to support the assumptions of a predominately heterotrophic driven respiration and respiratory rates unconstrained by primary production}

For a meaningful analysis of ER/GPP ratios and the derivation of MTE based predictions of the switch point temperature from net auto- to net heterotrophy (equation 5), we assume that respiration in the mesocosm is mainly driven by heterotrophic metabolism and is unconstrained by primary production, i.e. ER has in principle the potential to exhibit higher activation energies than GPP as predicted by MTE. To back up the made assumptions with data driven evidence we firstly established, if the difference in temperature dependence between ER and GPP is statistical significant and secondly estimated the background respiration as evidence for respiration independent from contemporaneous GPP.

\section{S6.1: Difference between the temperature sensitivity of ER and GPP}

Based on MTE we hypothesised that ER has a higher temperature sensitivity than GPP. Thus, if ER can increase with temperature unconstrained by GPP, we expect to find a higher average activation energy for ER than GPP. Ideally this difference is fortified by a statistical significant difference in the temperature sensitivity of the two metabolic types (ER, GPP). To test this we used ER and GPP as additional factors beside the treatments. The following basic linear mixed effect regression model (LMER) was tested ( $\mathrm{R}$ notation):

$\operatorname{lmer}\left(\ln (\right.$ metabolic rate $) \sim$ met $_{\text {type }} *$ invT $* \mathrm{~N} * \mathrm{D}+($ invT $\mid$ Country $)+\left(\right.$ invT $\mid$ Country: $\left.\mathrm{Id}_{\text {meso }}\right)+$ (1|Country:Month)) (S6.1)

, where met ${ }_{\text {type }}$ is the metabolic type (GPP or ER); invT is the inverse scaled temperature $\left(\mathrm{k}^{-1}\left(\mathrm{~T}_{\mathrm{c}}^{-1}-\right.\right.$ $\left.\mathrm{T}^{-1}\right)$ ); $\mathrm{N}$ is the nutrient level (mesotrophic $(\mathrm{L}=$ low) or eutrophic $(\mathrm{H}=$ high) $)$; is the depth $(1 \mathrm{~m}$ $(\mathrm{S}=$ sallow) or $2 \mathrm{~m}(\mathrm{D}=$ deep $)$ ); Country is a factor with 6 factor levels, i.e. one for each country; $\mathrm{Id}_{\text {meso }}$ serves as factor to uniquely identify all mesocosms, and Month is a factor with 5 factor levels (July - November).

We used likelihood ratio tests (LRT) as implemented in the "step" function of the "ImerTest" package (Kuznetsova et al., 2014) for stepwise backward model selection (random and fixed effects). Factor covariate interaction was probed using two-tailed t tests for pairwise comparisons of least-square-means over the inverse scaled temperature gradient in 0.001 steps. The tests were conducted using the standard settings of the "lsmeans" package (Lenth, 2016), i.e. p-values were adjusted for multiple testing based on the Tukey method and the significance level was set to 0.05.

The result confirms that the interaction between temperature and metabolic rate type (i.e. GPP or ER) is significant and gives evidence that ER had the potential to change with temperature unconstrained by GPP. Significant differences between ER and GPP are given in the temperature range between $\sim 23.0^{\circ} \mathrm{C}-29.3^{\circ} \mathrm{C}$ (significant higher ER) and $7.3^{\circ} \mathrm{C}-8.8^{\circ} \mathrm{C}$ (significant higher GPP, see S6 Table 1 and Figure S6.1). 
S6 Table 1: Results from linear mixed effect regression (equation S6.1) with metabolic type (i.e. ER or GPP) as additional factor. Int $=$ Intercept, invT $=$ inverse scaled temperature, Met $_{\text {type }}=$ metabolic type, $\mathrm{N}=$ nutrient level, $\mathrm{D}=$ depth. In brackets the reference factor level is given: $\mathrm{ER}=$ ecosystem respiration, $\mathrm{L}=\mathrm{Low} / \mathrm{mesotrophic}$ conditions, $\mathrm{S}=$ shallow depth $(1 \mathrm{~m})$. Confidence intervals were established based on likelihood profiles (function "confint.merMod" of the "Ime4" package (Bates et al.,2014)). The temperature range of significant difference between the metabolic types were established based on two-tailed t tests for pairwise comparisons of least-square-means.

\begin{tabular}{|c|c|c|c|c|c|c|c|c|}
\hline Response & Predictor & Estimate & $2.50 \%$ & $97.50 \%$ & Std. Error & T-value & P-value & Temperature range of sig. difference \\
\hline \multirow{4}{*}{ Metabolic rates } & invT & -0.52 & -0.96 & -0.09 & 0.21 & 4.9 & 0.05 & \\
\hline & $\operatorname{Met}_{\text {type }}(\mathrm{ER})$ & -0.03 & -0.09 & 0.03 & 0.03 & -0.91 & 0.36 & \\
\hline & $\mathrm{N}(\mathrm{L})$ & -0.52 & -0.62 & -0.41 & 0.05 & -9.89 & $<0.01$ & \\
\hline & $\operatorname{Met}_{\text {type }}(\mathrm{ER}): \operatorname{invT}$ & -0.09 & -0.16 & -0.02 & 0.03 & -2.53 & 0.01 & $\begin{array}{l}7.3^{\circ} \mathrm{C}-8.8^{\circ} \mathrm{C} \text { (GPP sig. higher) } \\
23.0^{\circ} \mathrm{C}-29.3^{\circ} \mathrm{C} \text { (ER sig. higher) }\end{array}$ \\
\hline
\end{tabular}

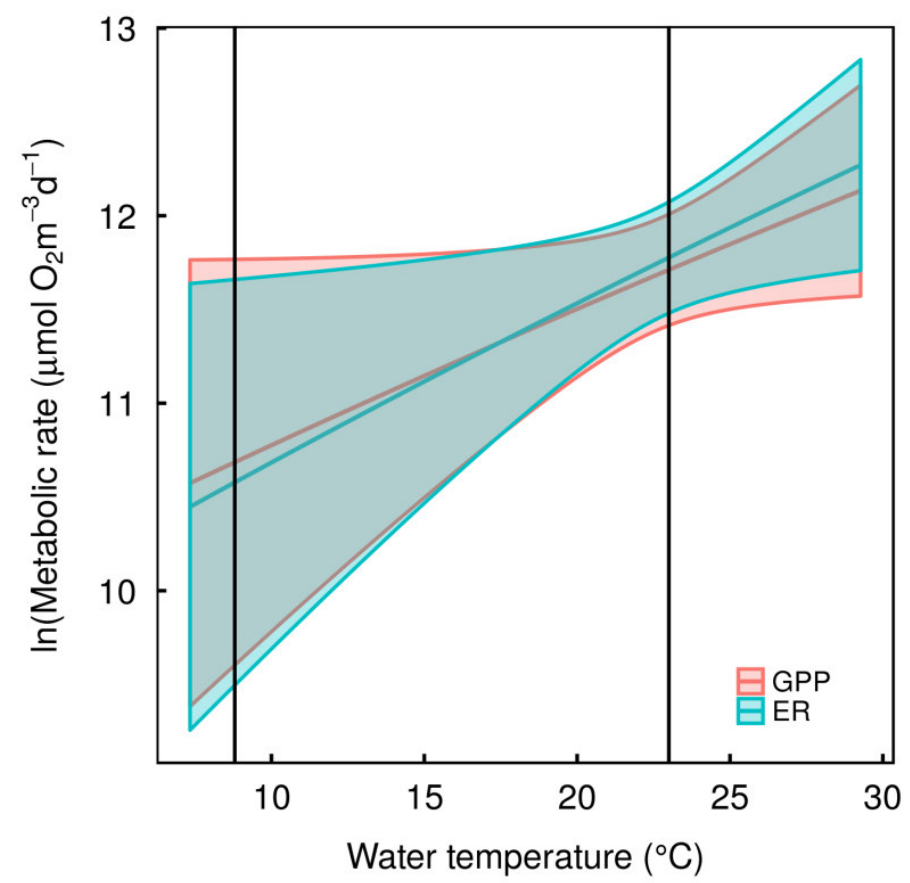

Figure S6.1: Least square means with $95 \%$ confidence intervals for gross primary production (GPP) and ecosystem respiration (ER). Vertical lines delimitate temperature ranges of significant difference between ER and GPP. GPP is significantly higher than ER in the temperature range between $7.3^{\circ} \mathrm{C}$ and $8.8^{\circ} \mathrm{C}$. ER is significantly higher than GPP in the temperature range between $23.0^{\circ} \mathrm{C}$ and $29.3^{\circ} \mathrm{C}$.

\section{S6.2: Coupling of respiration to GPP}

Coupling of respiration to GPP was analysed to identify background respiration and thus respiration unconstrained by GPP. We assessed background respiration following the approach presented in Solomon et al. (2013).

ER and GPP at $20^{\circ} \mathrm{C}\left(\mathrm{ER}_{20}, \mathrm{GPP}_{20}\right)$ were estimated as follows: 


$$
\begin{aligned}
& \mathrm{ER}_{20}=\frac{\mathrm{ER}(\mathrm{T})}{\Phi_{\mathrm{r}}^{(\mathrm{T}-20)}} \\
& \mathrm{GPP}_{20}=\frac{\mathrm{GPP}(\mathrm{T})}{\Phi_{\mathrm{p}}^{(\mathrm{T}-20)}}
\end{aligned}
$$

,where $\Phi_{\mathrm{r}}=1.09$ and $\Phi_{\mathrm{p}}=1.04$ are the temperature forcing for respiration and primary production respectively. $\Phi$ is estimated based on the average of two point form of the Arrhenius equation over a temperature interval of $0{ }^{\circ} \mathrm{C}$ to $30^{\circ} \mathrm{C}$ :

$\Phi=\frac{1}{303.15-273.15} \int_{273.15}^{303.15} \exp \left(\mathrm{E}\left(\mathrm{kT}_{1} \mathrm{~T}_{2}\right)^{-1}\right) \mathrm{dT}_{2}(\mathrm{~S} 6.4)$

, where $\mathrm{k}=8.6173324 * 10^{-5}$ is the Boltzmann constant, $\mathrm{E}$ is the activation energy with either $0.6 \mathrm{eV}$ (respiration) or $0.3 \mathrm{eV}$ (primary production), $\mathrm{T} 1=293.15 \mathrm{~K}\left(20^{\circ} \mathrm{C}\right)$ is the reference temperature and $\mathrm{T} 2=[273.15 \mathrm{~K}, 303.15 \mathrm{~K}]\left(\left[0{ }^{\circ} \mathrm{C}, 30^{\circ} \mathrm{C}\right]\right)$ is the considered temperature interval.

The relation between $\mathrm{ER}_{20}$ and $\mathrm{GPP}_{20}$ was analysed with linear mixed effects regression ("Ime" function of the "nlme" package (Pinheiro et al., 2015)). Model selection was based on AICc (Akaike information criterion with correction for finite sample size). 95\% confidence intervals at $\mathrm{GPP}_{20}=0$ were estimated using the R function "confint" of the "lsmeans" package (Lenth, 2016). The analysis was conducted in two ways. Firstly, background respiration was assessed for each treatment within country, using GPP at $20^{\circ} \mathrm{C}$, country and treatments as fixed effects and individual mesocosms (slope and intercept) as random effect to account for repeated measurements. The following models with highest AICc were used ("Ime" package notation):

$$
\begin{aligned}
& \text { lme }\left(\mathrm{ER}_{20} \sim \text { Country + D + GPP } 20+\mathrm{N}+\text { Country:D + Country:GPP } 20+\right. \\
& \left.\quad \text { Country:N + D:GPP } \mathrm{GP}_{20}+\text { Country:D:GPP } 20, \text { random }=\sim 1+\mathrm{GPP}_{20} \mid \mathrm{Id}_{\text {meso }}\right)
\end{aligned}
$$

,where $\mathrm{ER}_{20}$ and $\mathrm{GPP}_{20}$ are ecosystem respiration and gross primary production, respectively, standardised to $20^{\circ} \mathrm{C}$ (see equations S6.2 and S6.3), D is depth ( $\mathrm{S}=$ shallow, $\left.\mathrm{D}=\mathrm{Deep}\right), \mathrm{N}$ is the nutrient level $(\mathrm{H}=$ high, $\mathrm{L}=\mathrm{low}), \mathrm{Id}_{\text {meso }}$ is a unique identifier for each mesocosm.

Secondly, country and individual mesocosms nested in country (slope and intercept) were regarded as random effect, to estimated average background respiration per treatment, which parallels the level of analysis presented in the main text. The following models with highest AICc were used ("lme" package notation):

$$
\operatorname{lme}\left(\mathrm{ER}_{20} \sim \mathrm{GPP}_{20}+\mathrm{N}+\mathrm{D}, \text { random }=\sim \mathrm{GPP} 20 \mid \text { Country } / \mathrm{Id}_{\text {meso }}\right)
$$

,where $\mathrm{ER}_{20}$ and $\mathrm{GPP}_{20}$ are ecosystem respiration and gross primary production respectively, standardised to $20{ }^{\circ} \mathrm{C}$ (see equations S6.2 and S6.3), D is depth ( $\mathrm{S}=$ shallow, $\mathrm{D}=\mathrm{Deep}$ ), $\mathrm{N}$ is the nutrient level $(\mathrm{H}=$ high, $\mathrm{L}=\mathrm{low}), \mathrm{Id}_{\text {meso }}$ is a unique identifier for each mesocosm.

Country-wise analysis showed that not all treatments within countries had positive background respiration or confidence intervals above zero (see S6 Table 2). Yet, no correlation between amount of background respiration and magnitude of the activation energy for ER could be confirmed.

Treatment-wise analysis showed that on average all treatments had significant positive background respiration (see S6 Table 3 and figure S6.2).

Based on these results the assumptions of dominance of heterotrophic respiration and an ER unconstrained by GPP is justifiable. 
S6 Table 2: Estimated background respiration and 95\% confidence intervals per treatment within country. Estimates are based on linear mixed effect regression models (see equation S6.5). 95\% confidence intervals at $\mathrm{GPP}_{20}=0$ are based on least square means. $\mathrm{D}=$ deep $(2 \mathrm{~m}), \mathrm{S}=$ shallow (1m), $\mathrm{H}=$ high nutrient level (eutrophic), $\mathrm{L}=$ low nutrient level (mesotrophic), $\mathrm{SE}=$ Sweden, $\mathrm{CZ}=$ Czech Republic, GE = Germany, EE $=$ Estonia, TR $=$ Turkey and GR $=$ Greece .

\begin{tabular}{|c|c|c|c|c|}
\hline Country & Treatment & $2.50 \%$ & Prediction & $\mathbf{9 7 . 5 0 \%}$ \\
\hline \multirow[t]{4}{*}{ SE } & $\mathrm{DH}$ & 26.30 & 51.60 & 76.89 \\
\hline & $\mathrm{SH}$ & 102.64 & 152.87 & 203.09 \\
\hline & DL & 6.27 & 28.65 & 51.03 \\
\hline & SL & 82.95 & 129.92 & 176.89 \\
\hline \multirow[t]{4}{*}{$\mathrm{CZ}$} & $\mathrm{DH}$ & -6.79 & 34.58 & 75.96 \\
\hline & SH & 41.91 & 83.53 & 125.14 \\
\hline & DL & -29.46 & -5.39 & 18.68 \\
\hline & SL & 15.71 & 43.56 & 71.40 \\
\hline \multirow[t]{4}{*}{ GE } & $\mathrm{DH}$ & 56.11 & 89.69 & 123.28 \\
\hline & SH & 45.91 & 90.85 & 135.80 \\
\hline & DL & 27.84 & 50.65 & 73.47 \\
\hline & SL & 12.25 & 51.81 & 91.38 \\
\hline \multirow[t]{4}{*}{$\mathrm{EE}$} & $\mathrm{DH}$ & -32.90 & 2.02 & 36.93 \\
\hline & SH & -38.53 & -0.54 & 37.44 \\
\hline & DL & 2.39 & 23.58 & 44.76 \\
\hline & SL & -4.62 & 21.02 & 46.65 \\
\hline \multirow[t]{4}{*}{ TR } & $\mathrm{DH}$ & 21.31 & 53.27 & 85.24 \\
\hline & SH & -7.14 & 50.11 & 107.36 \\
\hline & DL & 9.44 & 33.39 & 57.35 \\
\hline & SL & -21.86 & 30.23 & 82.32 \\
\hline \multirow[t]{4}{*}{ GR } & $\mathrm{DH}$ & -42.24 & 5.72 & 53.67 \\
\hline & SH & -218.74 & -115.31 & -11.88 \\
\hline & DL & -17.75 & 13.25 & 44.26 \\
\hline & SL & -210.94 & -107.77 & -4.61 \\
\hline
\end{tabular}

S6 Table 3: Estimated background respiration and 95\% confidence intervals per treatment. Estimates are based on linear mixed effect regression models (see equation S6.6). 95\% confidence intervals at $\mathrm{GPP}_{20}=0$ are based on least square means. $\mathrm{D}=\operatorname{deep}(2 \mathrm{~m}), \mathrm{S}=$ shallow $(1 \mathrm{~m}), \mathrm{H}=$ high nutrient level (eutrophic) and $\mathrm{L}=$ low nutrient level (mesotrophic).

\begin{tabular}{|l|r|r|r|}
\hline Treatment & $\mathbf{2 . 5 0 \%}$ & Prediction & $\mathbf{9 7 . 5 0 \%}$ \\
\hline DH & 15.80 & 36.35 & 56.91 \\
\hline SH & 24.43 & 48.42 & 72.42 \\
\hline DL & 2.49 & 19.31 & 36.12 \\
\hline SL & 11.63 & 31.38 & 51.12 \\
\hline
\end{tabular}




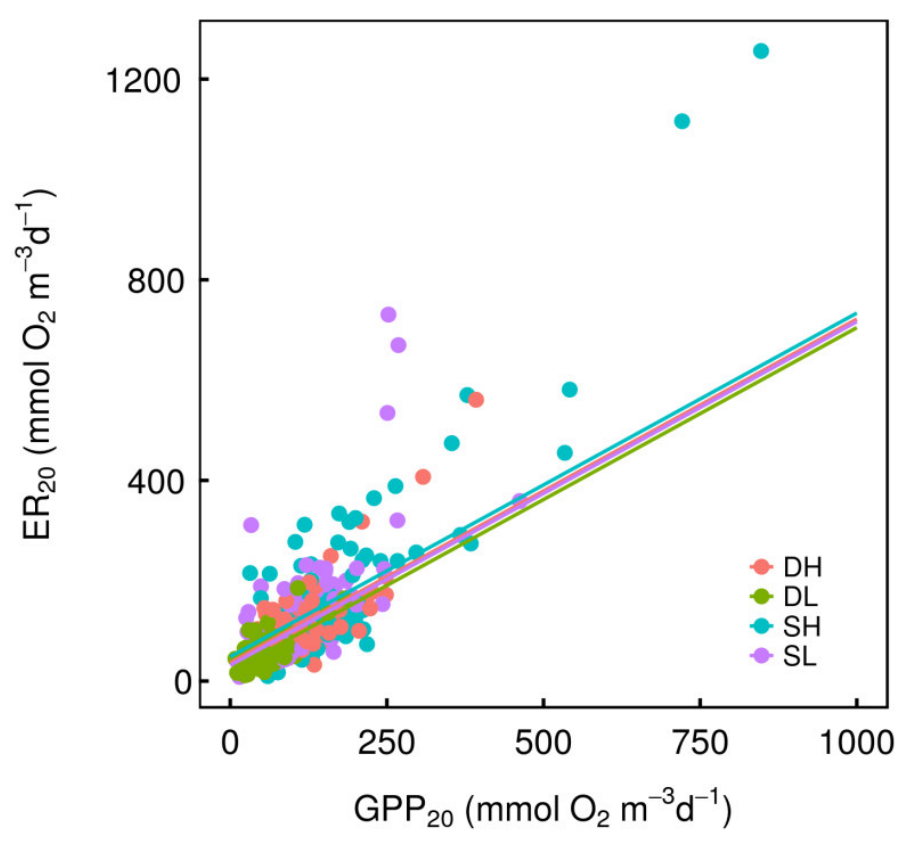

Figure S6.2: Treatment-wise coupling of ecosystem respiration $\left(\mathrm{ER}_{20}\right)$ to gross primary production $\left(\mathrm{GPP}_{20}\right)$ based on metabolic rates standardised to $20^{\circ} \mathrm{C}$ (see equation S6.2 and S6.3). Following Solomon et al. (2013) the slope of the relation quantifies coupling of respiration (respiration by autotrophs and respiration of autochthonous produced label organic matter by heterotrophs) to GPP, while the intercept quantifies the background respiration (respiration of recalcitrant autochthonous or surplus and allochthonous organic matter by heterotrophs; see also Solomon et al. (2013) Figure 1).

\section{S6 References}

Bates D, Maechler M, Bolker B, Walker S (2014)._lme4: Linear mixed-effects models using Eigen and S4_. R package version 1.1-7, <URL:http://CRAN.R-project.org/package=lme4>.

Kuznetsova A, Brockhoff PB and Christensen RHB (2014). lmerTest: Tests in Linear Mixed Effects Models. R package version 2.0-20. https://CRAN.R-project.org/package=lmerTest

Lenth RV (2016). Least-Squares Means: The R Package lsmeans. Journal of Statistical Software, 69(1), 1-33. doi:10.18637/jss.v069.i01

Pinheiro J, Bates D, DebRoy S, Sarkar D and R Core Team (2015). _nlme: Linear and Nonlinear Mixed Effects Models_. R package version 3.1-120, <URL: http://CRAN.Rproject.org/package $=$ nlme $>$.

Solomon CT, Bruesewitz DA, Richardson DC et al. (2013) Ecosystem respiration: Drivers of daily variability and background respiration in lakes around the globe. Limnology and Oceanography, 58, 849-866. 


\section{S7 Separation of the effects of the collinear variables temperature and daylight length on gross primary production (GPP)}

In the experiment mean daylight length (DayL) is highly correlated with water temperature and thus with inverse scaled temperature (invT). Both variables in turn are correlated with GPP (see S7 Table 1). Under these circumstances it is problematic to use the two variables in the same regression model and the estimated coefficients could not be interpreted independently from each other. These problems can already arise at comparable low values of the variance inflation factor (VIF), which in our case is 1.64 (only defined and thus established for simple linear regression models) (Graham, 2003).

S7 Table 1: Person- and Spearmans's rank correlation coefficient between inverse scaled water temperature (invT), daylight length (DayL) and logarithmised gross primary production $(\ln (\mathrm{GPP}))$

\begin{tabular}{|l|l|l|}
\hline Variables & Pearson correlation coefficient & Spearman's rank correlation coefficient \\
\hline InvT - mean DayL & -0.62 & -0.55 \\
\hline $\ln (\mathrm{GPP})$ - invT & -0.57 & -0.55 \\
\hline $\ln (\mathrm{GPP})$ - mean DayL & 0.26 & 0.21 \\
\hline
\end{tabular}

To consolidate the relevance and importance of temperature apart from daylight length as driver of GPP we used three different approaches: (1) semi-partial correlation (results presented in the main text), (2) two separate models with standardized variables and (3) residual regression (Graham 2003; Dormann et al., 2012). To estimate the activation energy without the potentially confounding influence of daylight length GPP per daylight hour was used (results presented in the main text).

The results from all the three different approaches coherently pinpoint both, daylight length and water temperature, as important variables (see Table 4 and S7 Table 2). Yet water temperature seems to have a slightly higher effect size and explanatory power compared to daylight length. This is also the case when the commonly explained variance is removed and only the partial explanatory power is compared, with the potential exception for deep high nutrient mesocosms (see Table 4). Daylight length is significantly more important in deep compared to shallow mesocosms (S7 Table 2),

\section{S7 References}

Dormann CF, Elith J, Bacher S, Buchmann C, Carl G, Carré G, Marquéz JRG, Gruber B, Lafourcade B, Leitão PJ, Münkemüler T, McClean C, Osborne PE, Reineking B, Schröder B, Skidmore AK, Zurell D, Lautenbach S (2012) Collinearity: A review of methods to deal with it and a simulation study evaluating their performance. Ecography 35, no - no

Graham MH (2003) Confronting multicollinearity in ecological multiple regression. Ecology, 84(11), $2809-2815$. 
S7 Table 2: Results from the two separate linear mixed effect and the residual regression models based on standardized variables to separating the relative importance of the two collinear predictors daylight length $\left(\right.$ DayL $\left._{S t d}\right)$ and inverse scaled water temperature (inv $\mathrm{T}_{\mathrm{Std}}$ ) on gross primary production (GPP).

\begin{tabular}{|c|c|c|c|c|c|c|}
\hline & Response & Predictor & Estimate & P-value & $\mathrm{R}^{2}$ conditional & $\mathrm{R}^{2}$ marginal \\
\hline \multirow{4}{*}{$\begin{array}{l}\text { Separate mixed effect models with standardized } \\
\text { variables }\end{array}$} & $\begin{array}{l}\text { Models } \\
\text { for } \\
\text { GPP }\end{array}$ & \multicolumn{5}{|c|}{ 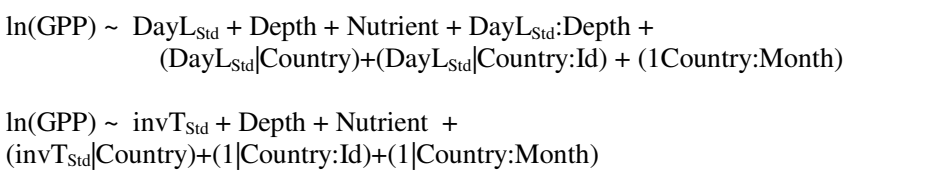 } \\
\hline & \multirow{3}{*}{$\ln (\mathrm{GPP})$} & DayL $_{\text {Std }}$ & 0.34 (deep) & 0.04 & 0.3 & 0.84 \\
\hline & & DayL $_{\text {Std }}$ : Depth & -0.13 (shallow) & 0.00 & & \\
\hline & & $\operatorname{invT}_{\mathrm{Std}}$ & -0.43 & 0.02 & 0.5 & 0.8 \\
\hline \multirow{5}{*}{ Residual regression } & Models & \multirow{2}{*}{\multicolumn{5}{|c|}{ 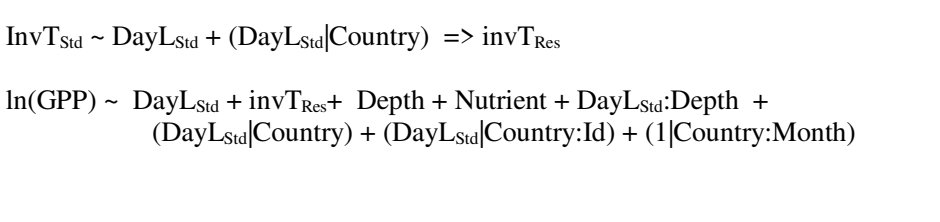 }} \\
\hline & & & & & & \\
\hline & \multirow{3}{*}{$\ln (\mathrm{GPP})$} & DayL $_{S t d}$ & 0.34 (deep) & 0.03 & \multirow{3}{*}{0.33} & \multirow{3}{*}{0.84} \\
\hline & & DayL $_{\text {Std }}$ : Depth & -0.14 (shallow) & 0 & & \\
\hline & & $\operatorname{invT}_{\text {Res }}$ & -0.77 & 0 & & \\
\hline
\end{tabular}




\title{
Appendix S8: Simulation based assessment on the value of average vs. linear mixed effect regression approaches for the analysis of temperature sensitivity and absolute metabolic rate for the particular experimental design of a standardised pan-European mesocosm experiment
}

\begin{abstract}
We conducted a simulation to test the robustness of linear mixed effects regressions (LMER) to estimate average temperature sensitivity of metabolic rates and absolute metabolic rates in the special situation of our experimental set-up. For this purpose, we emulated the random structure of our experimental data, i.e. we simulated six countries with different temperature ranges and within those countries, different experimental units. We simulated 7 scenarios in regard to the behaviour of temperature sensitivity and absolute metabolic rate among countries. In scenario 1 we assume that all countries have the same generic temperatures sensitivity and absolute metabolic rate and random variation in these quantities only occurs on the level of the unit, in scenario $2-4$ we consider random variation among countries for the absolute metabolic rate and or temperature sensitivity and in scenario $5-7$ we assume systematic changes with average temperature in one or both of these quantities. An LMER model, comparable with the one used in the manuscript, was then applied to each of this scenarios to estimate one overall average temperature sensitivity and absolute metabolic rate. While the scenarios $1-4$ concur with the LMER assumption of normally distributed random effects, this assumption is being violated in the scenarios $5-7$. Moreover, because of overlapping but distinct temperature ranges for each country, country-wise average temperatures may correlate by chance or systematically with country-wise average metabolic rates or temperature sensitivity. Such a correlation could potentially confound the estimated overall averages for temperature sensitivity and absolute metabolic rate. We also compared the performance of the LMER against two potentially alternative approaches based on average temperatures and metabolic rates. We found that the LMER reliably and universally estimates the true average temperature sensitivities and absolute metabolic rates. This was even the case for scenarios 5-7, where we consistently violate LMER assumptions. On the other hand, the estimates of the tested average-based approaches were heavily confounded by chance or systematic correlation between country-wise average temperatures and absolute metabolic rates and to a lesser extent average temperature and temperature sensitivity. None of the 3 tested approaches were able to quantify or at least detect the simulated systematic changes in absolute metabolic rates and or temperature sensitivity. In summary, the simulation results demonstrate clearly that the chosen LMER approach is suitable for robustly and meaningfully estimating average temperature sensitivity and absolute metabolic rates for the data from our experiment, if we assume random variation of these quantities among countries around an average value. However, even though the LMER approach was robust against the violation of the assumption of normally distributed random effects, the estimated average values under the assumption of systematic change are of questionable informational value, and an evaluation of trends in country-wise established temperature sensitivities and absolute metabolic rates seems relevant in this situation. For our data, however, we did not find any evidence for systematic changes with average temperature neither for temperature sensitivity nor absolute metabolic rate (S4). However, the average-based approaches are contraindicated in connection with our data where the specific experimental set up can give rise to chance correlations between average temperatures and absolute metabolic rates and or temperature sensitivity.
\end{abstract}




\section{Contents}

1 The aim of the simulation 4

2 Method $\mathbf{5}$

2.1 Simulation set-up . . . . . . . . . . . . . . . . . . . . . . 5

2.2 Analysis of simulation results . . . . . . . . . . . . . . . . . . . . . . . 6

2.2.1 Image: "Simulated metabolic rates and respective averages" . . . . . . . . . . . . 6

2.2.2 Image: "Log transformed simulated metabolic rate, averages and model predictions" . 6

2.2.3 Image: "Correlation of simulated parameters $A_{c, i}$ and $E_{c, i}$ with temperature" . . . . 7

2.2.4 Visual comparison between the parameters $\hat{A}$ and $\hat{E}$ estimated by the three different approaches and the simulated parameters $\overline{A_{c, i}}$ and $\overline{A_{c, i}}$. . . . . . . . . . . . . . . . . 7

2.2.4.1 Comparison of the distribution of the differences between estimated and simulated values by the three approaches based on 1000 simulation runs. . . 7

2.2.4.2 Comparison of the distribution of the estimated and simulated values based on 1000 simulation runs . . . . . . . . . . . . . . . . . . 7

2.2.5 Analysis of the cause for the deviaten between estimated vs. simulated density distribu-

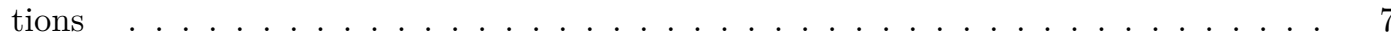

3 Scenario one: All entities have the same generic activation energy $E_{c}$ and intercept $A_{c} 9$

3.1 Description and assumptions of scenario one . . . . . . . . . . . . . . . . . . . . . .

3.2 Summary results scenario one: . . . . . . . . . . . . . . . . . . . . . . . 11

3.3 Scenario one: Comparison between $\overline{E_{c, i}}$ and $\overline{\ln A_{c, i}}$ and $\hat{E}$ and $\hat{A}$ from the 3 different estimation

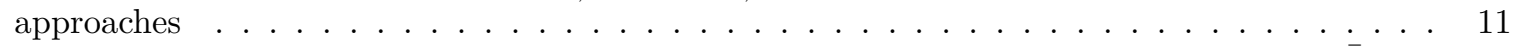

3.4 Scenario one: Detailed results on the influnece of chance correlation of $\ln A_{c, i} E_{c, i}$ with $\bar{T}_{c}$ on the estimates of $\hat{E}$ and $\hat{A} \ldots \ldots \ldots \ldots \ldots \ldots$

3.4.1 Log-first approach - Best model to explain $\hat{E}$ and $\hat{A}$ based on $\overline{E_{c, i}}, \overline{\ln A_{c, i}}, \beta_{E_{c, i}}$ and $\beta_{A_{c, i}} 13$

3.4.2 Average-first approach - Best model to explain $\hat{E}$ and $\hat{A}$ based on $\overline{E_{c, i}}, \overline{\ln A_{c, i}}, \beta_{E_{c, i}}$ and $\beta_{A_{c, i}}$. . . . . . . . . . . . . . . . . . . . . . . . . . . . . . . 15

3.4.3 LMER approach - Best model to explain $\hat{E}$ and $\hat{A}$ based on $\overline{E_{c, i}}, \overline{\ln A_{c, i}}, \beta_{E_{c, i}}$ and $\beta_{A_{c, i}} 17$

4 Scenario two: All entities have the same generic activation energy $E_{c}$ but have a variable intercept $A_{c}$

4.1 Description and assumptions of scenario two . . . . . . . . . . . . . . . . . . 19

4.2 Summary results scenario two: . . . . . . . . . . . . . . . . . . . . . . . . . 21

4.3 Scenario two: Comparison between $\overline{E_{c, i}}$ and $\overline{\ln A_{c, i}}$ and $\hat{E}$ and $\hat{A}$ from the 3 different estimation

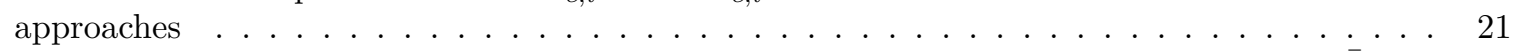

4.4 Scenario two: Detailed results on the influnece of chance correlation of $\ln A_{c, i} E_{c, i}$ with $\bar{T}_{c}$ on the estimates of $\hat{E}$ and $\hat{A} \ldots \ldots \ldots \ldots \ldots \ldots \ldots \ldots$

4.4.1 Log-first approach - Best model to explain $\hat{E}$ and $\hat{A}$ based on $\overline{E_{c, i}}, \overline{\ln A_{c, i}}, \beta_{E_{c, i}}$ and $\beta_{A_{c, i}} 23$

4.4.2 Average-first approach - Best model to explain $\hat{E}$ and $\hat{A}$ based on $\overline{E_{c, i}}, \overline{\ln A_{c, i}}, \beta_{E_{c, i}}$ and $\beta_{A_{c, i}}$. . . . . . . . . . . . . . . . . . . . . . . . . . . . 25

4.4.3 LMER approach - Best model to explain $\hat{E}$ and $\hat{A}$ based on $\overline{E_{c, i}}, \overline{\ln A_{c, i}}, \beta_{E_{c, i}}$ and $\beta_{A_{c, i}} 27$

5 Scenario three: All entities have the same generic absolute metabolic rate $A_{c}$ but variable $E_{c} \quad \mathbf{2 9}$

5.1 Description and assumptions of scenario three . . . . . . . . . . . . . . . . . . 29

5.2 Summary results scenario three. . . . . . . . . . . . . . . . . . . . . . . . . 31

5.3 Scenario three: Comparison between $\overline{E_{c, i}}$ and $\overline{\ln A_{c, i}}$ and $\hat{E}$ and $\hat{A}$ from the 3 different estimation approaches . . . . . . . . . . . . . . . . . . . . . . . . . 31

5.4 Scenario three: Detailed results on the influnece of chance correlation of $\ln A_{c, i} E_{c, i}$ with $\bar{T}_{c}$ on the estimates of $\hat{E}$ and $\hat{A} \ldots \ldots \ldots \ldots \ldots$ 5.4.1 Log-first approach - Best model to explain $\hat{E}$ and $\hat{A}$ based on $\overline{E_{c, i}}, \overline{\ln A_{c, i}}, \beta_{E_{c, i}}$ and $\beta_{A_{c, i}} 33$ 
5.4.2 Average-first approach - Best model to explain $\hat{E}$ and $\hat{A}$ based on $\overline{E_{c, i}}, \overline{\ln A_{c, i}}, \beta_{E_{c, i}}$

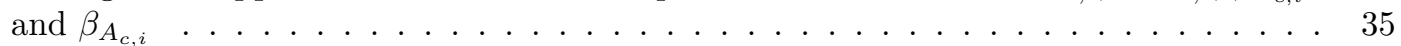

5.4.3 LMER approach - Best model to explain $\hat{E}$ and $\hat{A}$ based on $\overline{E_{c, i}}, \overline{\ln A_{c, i}}, \beta_{E_{c, i}}$ and $\beta_{A_{c, i}} 37$

6 Scenario four: All entities have variable absolute metabolic rates $A_{c}$ and activation energies $E_{c}$

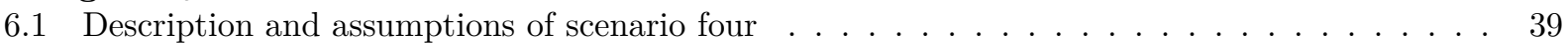

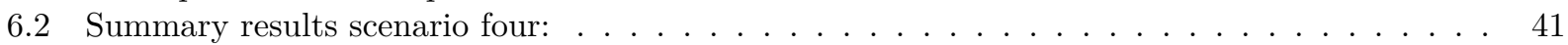

6.3 Scenario four: Comparison between $\overline{E_{c, i}}$ and $\overline{\ln A_{c, i}}$ and $\hat{E}$ and $\hat{A}$ from the 3 different estimation

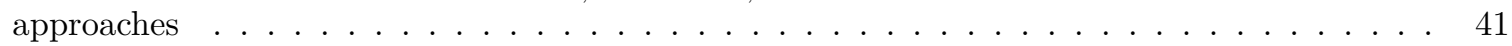

6.4 Scenario four: Detailed results on the influnece of chance correlation of $\ln A_{c, i} E_{c, i}$ with $\bar{T}_{c}$ on

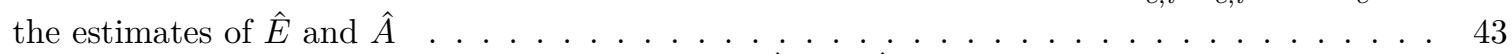

6.4.1 Log-first approach - Best model to explain $\hat{E}$ and $\hat{A}$ based on $\overline{E_{c, i}}, \overline{\ln A_{c, i}}, \beta_{E_{c, i}}$ and $\beta_{A_{c, i}} 43$

6.4.2 Average-first approach - Best model to explain $\hat{E}$ and $\hat{A}$ based on $\overline{E_{c, i}}, \overline{\ln A_{c, i}}, \beta_{E_{c, i}}$

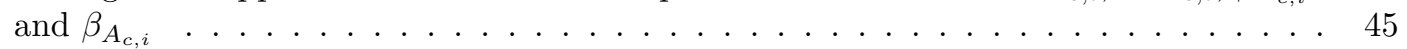

6.4.3 LMER approach - Best model to explain $\hat{E}$ and $\hat{A}$ based on $\overline{E_{c, i}}, \overline{\ln A_{c, i}}, \beta_{E_{c, i}}$ and $\beta_{A_{c, i}} 47$

7 Scenario five: All entities are allowed to have variable $E_{c}$ and $A_{c}$. Where $E_{c}$ are negatively correlated with $\bar{T}_{c}$

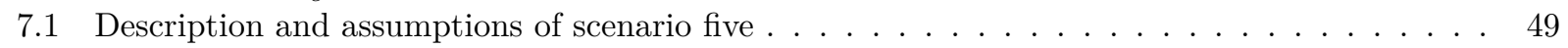

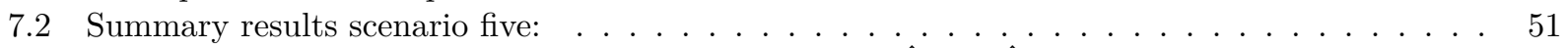

7.3 Scenario five: Comparison between $\overline{E_{c, i}}$ and $\overline{\ln A_{c, i}}$ and $\hat{E}$ and $\hat{A}$ from the 3 different estimation

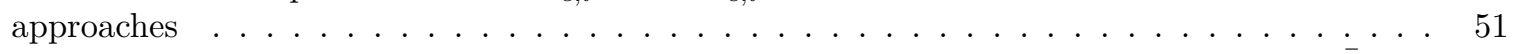

7.4 Scenario five: Detailed results on the influnece of chance correlation of $\ln A_{c, i} E_{c, i}$ with $\bar{T}_{c}$ on

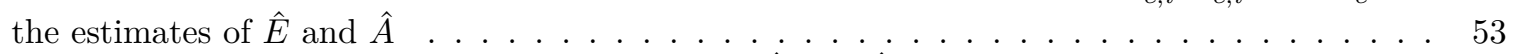

7.4.1 Log-first approach - Best model to explain $\hat{E}$ and $\hat{A}$ based on $\overline{E_{c, i}}, \overline{\ln A_{c, i}}, \beta_{E_{c, i}}$ and $\beta_{A_{c, i}} 53$

7.4.2 Average-first approach - Best model to explain $\hat{E}$ and $\hat{A}$ based on $\overline{E_{c, i}}, \overline{\ln A_{c, i}}, \beta_{E_{c, i}}$

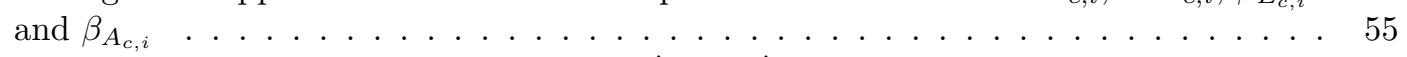

7.4.3 LMER approach - Best model to explain $\hat{E}$ and $\hat{A}$ based on $\overline{E_{c, i}}, \overline{\ln A_{c, i}}, \beta_{E_{c, i}}$ and $\beta_{A_{c, i}} 57$

8 Scenario six: All entities have variale $A_{c}$ and $E_{c}$. $A_{c}$ 's systematically increase with increasing $\bar{T}_{c}$

8.1 Description and assumptions of scenario six $\ldots \ldots \ldots \ldots \ldots \ldots \ldots$

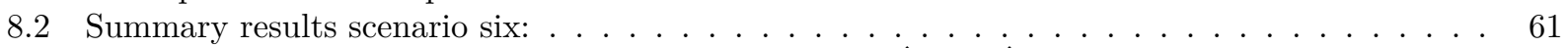

8.3 Scenario six: Comparison between $\overline{E_{c, i}}$ and $\overline{\ln A_{c, i}}$ and $\hat{E}$ and $\hat{A}$ from the 3 different estimation

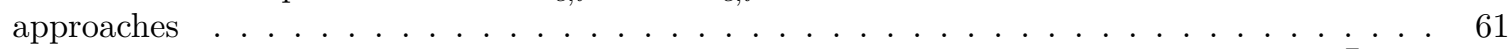

8.4 Scenario six: Detailed results on the influnece of chance correlation of $\ln A_{c, i} E_{c, i}$ with $\bar{T}_{c}$ on

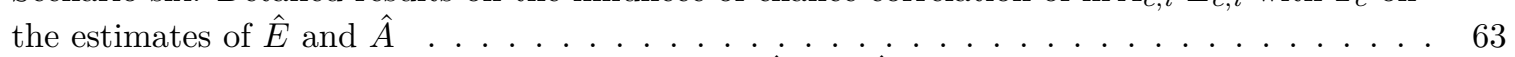

8.4.1 Log-first approach - Best model to explain $\hat{E}$ and $\hat{A}$ based on $\overline{E_{c, i}}, \overline{\ln A_{c, i}}, \beta_{E_{c, i}}$ and $\beta_{A_{c, i}} 63$

8.4.2 Average-first approach - Best model to explain $\hat{E}$ and $\hat{A}$ based on $\overline{E_{c, i}}, \overline{\ln A_{c, i}}, \beta_{E_{c, i}}$

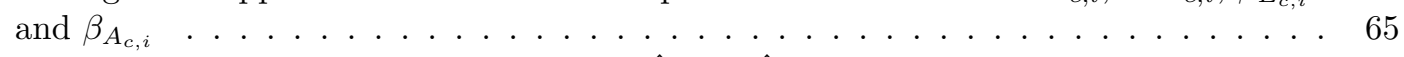

8.4.3 LMER approach - Best model to explain $\hat{E}$ and $\hat{A}$ based on $\overline{E_{c, i}}, \overline{\ln A_{c, i}}, \beta_{E_{c, i}}$ and $\beta_{A_{c, i}} 67$

9 Scenario seven: All entities are allowed to have variable $A_{c}$ and $E_{c}$ which systematically increase respectively decrease with $\bar{T}$

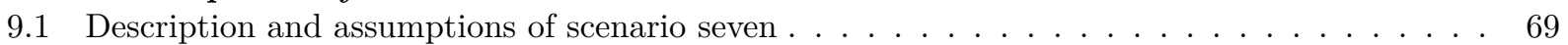

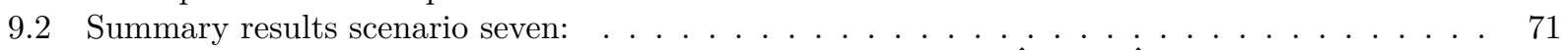

9.3 Scenario seven: Comparison between $\overline{E_{c, i}}$ and $\overline{\ln A_{c, i}}$ and $\hat{E}$ and $\hat{A}$ from the 3 different

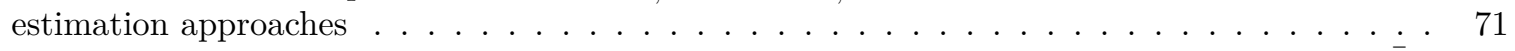

9.4 Scenario seven: Detailed results on the influnece of chance correlation of $\ln A_{c, i} E_{c, i}$ with $\bar{T}_{c}$

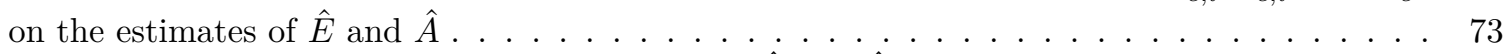
9.4.1 Log-first approach - Best model to explain $\hat{E}$ and $\hat{A}$ based on $\overline{E_{c, i}}, \overline{\ln A_{c, i}}, \beta_{E_{c, i}}$ and $\beta_{A_{c, i}} 73$ 
9.4.2 Average-first approach - Best model to explain $\hat{E}$ and $\hat{A}$ based on $\overline{E_{c, i}}, \overline{\ln A_{c, i}}, \beta_{E_{c, i}}$

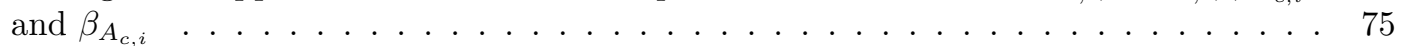

9.4.3 LMER approach - Best model to explain $\hat{E}$ and $\hat{A}$ based on $\overline{E_{c, i}}, \overline{\ln A_{c, i}}, \beta_{E_{c, i}}$ and $\beta_{A_{c, i}} 77$

\section{The aim of the simulation}

A pan-European standardised mesocosm experiment was conducted to elucidate how metabolic rates change in response to increased temperatures. The experiment was conducted in parallel in six countries from Sweden to Greece and run from May to November, covering a temperature range from $7-29^{\circ} \mathrm{C}$. A monthly measurement scheme was applied.

Aim of this simulation was to emulate data from this experimental set-up with particular focus on the reproduction of the random structure of the data implied by the set-up. This data was used to evaluate how reliable a linear mixed effect regression approach (LMER) is able to retrieve biologically meaningful information about the activation energies and absolute metabolic rates realized by the experimental set-up. The performance of LMER was tested under different perceivable hypotheses regarding the temperature sensitivity of metabolic rates and absolute metabolic rates. Further, results from LMER were compared to results based on two different average approaches.

In all simulated scenarios a non-linear relation between water temperature and metabolic rates was assumed, which in a temperature range from approximately 0 to $30{ }^{\circ} \mathrm{C}$ can be reasonable described by the Arrhenius equation. Two general different behaviors of the temperature sensitivity are perceivable. Firstly, in accordance with the assumptions of the metabolic theory, there exist one generic temperature sensitivity, which in the single countries and mesocosms might vary randomly around this temperature sensitivity due to interactions or co variation of other drivers with temperature. Secondly, temperature sensitivity could be an adaptive property on individual, population or community level, thus that the temperature sensitivity systematically changes with the average temperature of the different experimental locations. 


\section{Method}

Table 1: Glossary of terms.

\begin{tabular}{|c|c|}
\hline Term & Description \\
\hline$c=1, \ldots, 6$ & Index of the simulated entities \\
\hline$i=1, \ldots, 16$ & Index of the simulated instances \\
\hline $\mathrm{T}$ & Temperature \\
\hline$T_{c}=\left[T_{c, \min }, \ldots, T_{c, \max }\right]$ & $\begin{array}{l}\text { Temperature range of entity } \mathrm{c} \text { with } T_{c, \min } \text { and } T_{c, \max } \text { as the minimal and } \\
\text { maximal temperature of this range. } \forall \mathrm{c} \text { it holds that } T_{c, \max }-T_{c, \min }=14 \\
{ }^{\circ} \mathrm{C} . T_{c} \text { is the same for all } \mathrm{i} \text { belonging to entity } \mathrm{c} \text {. }\end{array}$ \\
\hline$\overline{T_{c}}$ & Average temperature of entity $\mathrm{c}$ \\
\hline$T_{r}=15^{\circ} \mathrm{C}$ & Reference temperature \\
\hline A & Generic absolute metabolic rate at $T_{r}$ \\
\hline $\mathrm{E}$ & Generic activation energy \\
\hline$A_{c} \sim N(13,4)$ & Absolute metabolic rate at $T_{r}$ of entity c \\
\hline$E_{c} \sim N(0.6,0.5)$ & Activation energy of entity c. Values of $E_{c}$ are limited to $E_{c} \geq 0$ \\
\hline$A_{c, i} \sim N\left(A_{c}, 0.4\right)$ & Simulated absolute metabolic rate at $T_{r}$ of instance i of entity c. \\
\hline$E_{c, i} \sim N\left(E_{c}, 0.1\right)$ & Simulated activation energy of instance i of entity c. \\
\hline$\overline{\ln A_{c, i}}$ & Average of the logarithmised $A_{c, i}$ over all simulated entities c and instances i \\
\hline$\overline{E_{c, i}}$ & Average $E_{c, i}$ over all simulated entities c and instances i \\
\hline$\hat{A}$ & Estimates of $\overline{\ln A_{c, i}}$ based on one of the three tested approaches \\
\hline$\hat{E}$ & Estimates of $\overline{E_{c, i}}$ based on one of the three tested approaches \\
\hline \multirow[t]{2}{*}{$M_{c, i}(T)$} & Metabolic rate of entity $\mathrm{c}$ and instance $\mathrm{i}$ at temperature $\mathrm{T}$. \\
\hline & $M_{c, i}(T)=A_{c, i} \cdot \exp \left(\frac{-E_{c, i}}{k} \cdot\left(\frac{1}{T}-\frac{1}{T_{r}}\right)\right)+\epsilon$ with $\epsilon \sim N(0,1)$ \\
\hline$\epsilon \sim N(0,1)$ & Gaussian random noise \\
\hline$\beta_{A_{c, i}}$ & $\begin{array}{l}\text { Regression coefficient quantifying the relation between the simulated absolute } \\
\text { metabolic rates } A_{c, i} \text { and } \overline{T_{c}}\end{array}$ \\
\hline$\beta_{E_{c, i}}$ & $\begin{array}{l}\text { Regression coefficient quantifying the relation between the simulated } \\
\text { activation energies } E_{c, i} \text { and } \overline{T_{c}}\end{array}$ \\
\hline$k=8.62 \cdot 10^{-5}\left[\mathrm{eVK}^{-1}\right]$ & Bolzmann constant \\
\hline
\end{tabular}

\subsection{Simulation set-up}

Each simulation run considered six entities $c=1, \ldots, 6$, which were represented by sixteen instances $i=1, \ldots, 16$, comparable to the experimental set-up which comprised six countries each with sixteen mesocosms.

For each entity $c$ a random temperature range of $14^{\circ} \mathrm{C}$ was drawn in a potential temperature range between 0 and $35{ }^{\circ} \mathrm{C}$.

In scenario 1 - 4 each of the entities $c$ got assigned a randomly drawn activation energy $E_{c} \sim N(0.6,0.5)$ and absolute metabolic rate $A_{c} \sim N(13,4)$ at reference temperature $T_{r}$. The reference temperature $T_{r}$ was set to $15{ }^{\circ} \mathrm{C}$. Depending on the scenario each entity had the same or different $A_{c}$ and $E_{c}$ values (see details of scenarios 1-4 below). Different $E_{c}$ and $A_{c}$ for the entities $c$ represented randomly distributed deviation from a generic absolute metabolic rate $A$ and a generic activation energy $E$ due to other factors which have a influence on metabolic rates and interact or correlated with temperature. However these factors were assumed to vary randomly between the entities. Based on the randomly drawn $A_{c}$ and $E_{c}$, each instance $i$ of entity $c$ was a signed an absolute metabolic rate $A_{c, i} \sim N\left(A_{c}, 0.4\right)$ and an activation energy $E_{c, i} \sim N\left(E_{c}, 0.1\right)$.

In scenario 5 - 7 the effect of systematic changes in $A_{c}$ and or $E_{c}$ with increasing average temperature $\overline{T_{c}}$ were simulated. Without loss of generality $E_{c}$ was assumed to decrease and $A_{c}$ to increase with increasing $\overline{T_{c}}$. Increase of $A_{c}$ and decrease of $E_{c}$ with $\overline{T_{c}}$ was simulated by sorting the randomly drawn values of $E_{c}$ and $A_{c}$ decreasingly and increasingly respectively and thus assign them to the temperature ranges sorted by 
increasing $\overline{T_{c}}$, i.e. the highest temperature range was associated with the smallest $E_{c}$ and the highest $A_{c}$ and the lowest temperature range with the highest $E_{c}$ and smallest $A_{c}$, etc.. $E_{c, i}$ and $A_{c, i}$ values for the instances were than generated as described above.

The thus established $T_{c}, A_{c, i}$ and $E_{c, i}$ were used to simulate temperate dependent metabolic rates $M_{c, i}(T)$ for each instance $i$ of entity $c$ according to the Arrhenius equation:

$$
M_{c, i}(T)=A_{c, i} \cdot \exp \left(\frac{-E_{c, i}}{k} \cdot\left(\frac{1}{T}-\frac{1}{T_{c}}\right)\right)+\epsilon
$$

, where $k$ is the Bolzmann constant and $\epsilon \sim N(0,1)$ is added random noise.

The relation between $M_{c, i}$ and $T$ as described above can be linearized by taking the logarithm of both side:

$$
\ln M_{c, i}(T)=\ln A_{c, i}-\frac{E_{c, i}}{k} \cdot\left(\frac{1}{T}-\frac{1}{T_{c}}\right)
$$

Linear regression can than be used to estimate an average metabolic rate at reference temperature $\overline{\ln A_{c, i}}(\hat{A})$ and an average activation energy $\overline{E_{c, i}}(\hat{E})$ based on $\ln M_{c, i}(T)$ from all entities $c$ and instance $i$.

Estimation of $\hat{E}$ and $\hat{A}$ was conducted with three different approaches:

1. Linear mixed effect model (LMER) approach: The simulated $\ln M_{c, i}(T)$ values were used in a linear mixed effect model considering random effects for the slope and the intercept on the level of entity and instances. The random effects for instances were nested within entity. This approach is comparable to the on used in the manuscript to analyse the experiment data.

2. Average first approach: The simulated $\ln M_{c, i}(T)$ values were used first to estimate average absolute metabolic rates on the level of instances. The resulting averages were than log transformed. Similar, first the average temperature $\overline{T_{c}}$ was calculated. Based on those averages the average scaled inverse temperature was calculated. This average-first data was then used to estimate $\hat{E}$ and $\hat{A}$ based on a LMER, with random intercept on the level of entity.

3. Log first approach: The simulated $\ln M_{c, i}(T)$ values were first $\log$ transformed and than averaged. Similar, inverse scaled temperature was calculated based on $T_{c}$ and than average inverse scaled temperatures estimated. This log-first data was than used to estimate $\hat{E}$ and $\hat{A}$ based on a LMER approach with random intercept on the level of entity.

For each scenario and each of the three approaches 1000 simulation runes were conducted.

\subsection{Analysis of simulation results}

\subsubsection{Image: "Simulated metabolic rates and respective averages"}

In the first analysis plot, for each scenario the simulated metabolic rates $M_{c, i}(T)$ from one simulation run were shown exemplary (see for example figure 1). The lines represent the exponential relation between temperature and metabolic rates with out added noise. The dots show the final simulated data with noise. Added to the graph were the means calculated based on noiseless data (open square) and noisy data (closed triangle).

\subsubsection{Image: "Log transformed simulated metabolic rate, averages and model predictions"}

In the second analysis plot, a so called Arrhenius plot, the same exemplary noise free (line) and noisy (small dots) $\ln M_{c, i}(T)$ as in the previouse image 2.2.1 were depicted (see for example figure 2). Added to the 
Arrhenius plot were averages based on noiseless (closed symbols) and noisy (open symbols) data. Averages were calculated using either the Average-first (square) or in the Log-first (triangle) approach. In addition the resulting predicted metabolic rates based on the 3 different approaches to estimate $\hat{E}$ and $\hat{A}$ were depicted: LMER approach (continuous black line), Average-first approach (dotted line), Log-first approach (dashed line).

\subsubsection{Image: "Correlation of simulated parameters $A_{c, i}$ and $E_{c, i}$ with temperature"}

In the third analysis plot (see for example figure 3), the correlation between $E_{c, i}$ and $\overline{T_{c}}$ and $A_{c, i}$ and $\overline{T_{c}}$ was shown for each scenario and the same exemplary data as in the previous images, e.g. 2.2.1. For scenario $1-4$ this correlation is based on chance alone. The strength of the relation was quantified by linear regression and expressed in the regression coefficients:

$$
\begin{aligned}
& A_{c, i}=\alpha+\beta_{A_{c, i}} \cdot \overline{T_{c}} \\
& E_{c, i}=\alpha+\beta_{E_{c, i}} \cdot \overline{T_{c}}
\end{aligned}
$$

\subsubsection{Visual comparison between the parameters $\hat{A}$ and $\hat{E}$ estimated by the three different approaches and the simulated parameters $\overline{A_{c, i}}$ and $\overline{A_{c, i}}$}

2.2.4.1 Comparison of the distribution of the differences between estimated and simulated values by the three approaches based on 1000 simulation runs.

In the fourth analysis plot, for each of the three approaches and each simulation run the differences $\overline{A_{c, i}}-\hat{A}$ and $\overline{E_{c, i}}-\hat{E}$ were calculated. The distributions of the differences from the three approaches were compared with the help of histograms (see for example figure 4). Ideally, the difference distribution is centred at zero with a small standard variation.

2.2.4.2 Comparison of the distribution of the estimated and simulated values based on 1000 simulation runs

In the fifth analysis plot, the density distributions of the estimated $(\hat{A}$ and $\hat{E})$ and simulated $\left(\overline{A_{c, i}}\right.$ and $\left.\overline{E_{c, i}}\right)$ parameters were depicted. Comparison of the density distributions allows to asses how well the three different estimation procedures were able to reproduce the distribution of the simulated parameters (see for example figure 5). For scenarios were either $A_{c, i}$ or $E_{c, i}$ was variable between entities and thus their existed the possibility of chance correlation of this parameters with $\overline{T_{c}}$, also the density distribution of $\beta_{A_{c, i}}$ and $\beta_{E_{c, i}}$ were added (see next paragraph 2.2.5, as well as equation 5 and 6 ). Ideally the density distribution of a estimated parameter is congruent with the one from the simulated parameter.

\subsubsection{Analysis of the cause for the deviaten between estimated vs. simulated density distri- butions}

In the sixth analysis plot, the potential causes for deviations between the density distribution of the estimated ( $\hat{A}$ and $\hat{E}$ ) and simulated $\left(\overline{A_{c, i}}\right.$ and $\overline{E_{c, i}}$ ) parameters were assessed. To investigate the influence of $\overline{A_{c, i}}, \overline{E_{c, i}}$, $\beta_{A_{c, i}}$ and $\beta_{E_{c, i}}$ on the estimated parameter values the following two regression models for each of the three tested approach were evaluated:

$$
\begin{aligned}
\hat{A}= & \alpha+\beta_{1} \overline{A_{c, i}}+\beta_{2} \overline{E_{c, i}}+\beta_{3} \beta_{A_{c, i}}+\beta_{4} \beta_{E_{c, i}}+\beta_{5} \overline{A_{c, i}}: \overline{E_{c, i}}+\beta_{6} \overline{A_{c, i}}: \beta_{A_{c, i}}+\beta_{7} \overline{A_{c, i}}: \beta_{E_{c, i}}+ \\
& \beta_{8} \overline{E_{c, i}}: \beta_{A_{c, i}}+\beta_{9} \overline{E_{c, i}}: \beta_{E_{c, i}}+\beta_{10} \beta_{A_{c, i}}: \beta_{E_{c, i}}
\end{aligned}
$$




$$
\begin{aligned}
\hat{E}= & \alpha+\beta_{1} \overline{A_{c, i}}+\beta_{2} \overline{E_{c, i}}+\beta_{3} \beta_{A_{c, i}}+\beta_{4} \beta_{E_{c, i}}+\beta_{5} \overline{A_{c, i}}: \overline{E_{c, i}}+\beta_{6} \overline{A_{c, i}}: \beta_{A_{c, i}}+\beta_{7} \overline{A_{c, i}}: \beta_{E_{c, i}}+ \\
& \beta_{8} \overline{E_{c, i}}: \beta_{A_{c, i}}+\beta_{9} \overline{E_{c, i}}: \beta_{E_{c, i}}+\beta_{10} \beta_{A_{c, i}}: \beta_{E_{c, i}}
\end{aligned}
$$

Model selection was based on AIC with the help of the R package MuMIn. For the best model in terms of AIC relative importance of the retained parameters was established by hierarchical partitioning (Chevan and Sutherland (1991) Hierarchical partitioning. The American Statistician 45(2):90-96) as implemented in the relaimpo-package (lmg method).

For each scenario a summary on the results of the performance of the three approaches was given, followed by tables (see for example tables 2, 3, 4 and 5) and images (see for example figure 6) documenting the detailed results. 


\section{Scenario one: All entities have the same generic activation en- ergy $E_{c}$ and intercept $A_{c}$}

\subsection{Description and assumptions of scenario one}

In this scenario it is assumed that there exists one generic temperature dependence $E_{c}=E \forall c$ and one generic absolute metabolic rate $A_{c}=A \forall c$. However, within the entities the $E_{c, i}$ and $A_{c, i}$ for each instance vary with Gaussian random noise $\left(A_{c, i} \sim N\left(A_{c}, 0.4\right)\right.$ and $\left.E_{c, i} \sim N\left(E_{c}, 0.1\right)\right)$. While a generic activation energy is also assumed by MTE, the fixed A are rather artificial in this scenario. Note, due to the fact that $E_{c}$ and $A_{c}$ are the same for all entities and $A_{c, i}$ and $E_{c, i}$ only vary with $\mathrm{N}(0,0.4)$ and $\mathrm{N}(0,0.1)$ Gaussian random noise within each entity the probability of an chance increase of $\overline{\ln A_{c, i}}$ or $\overline{E_{c, i}}$ with $\bar{T}_{c}$ is very limited in this scenario (see figure 3).

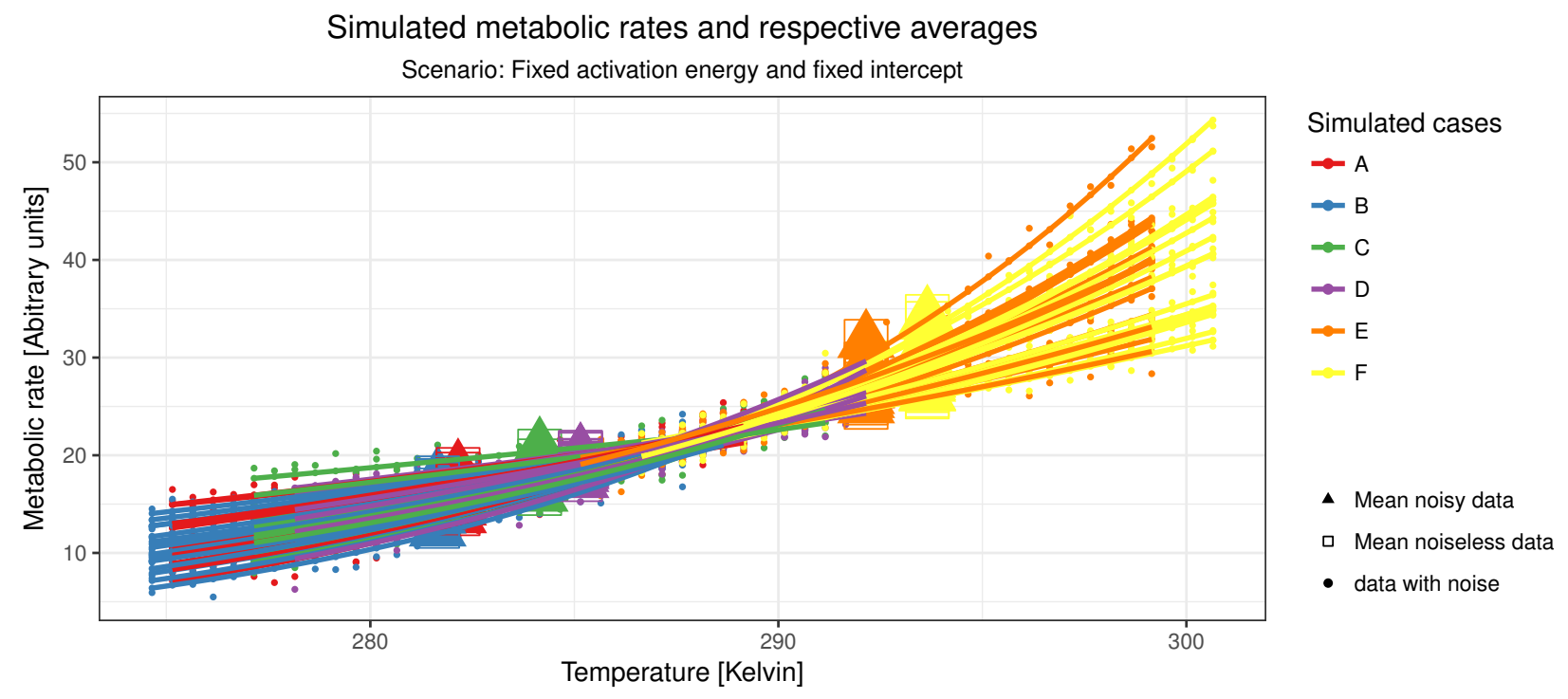

Figure 1: Examplary simulated raw data for scenario one 


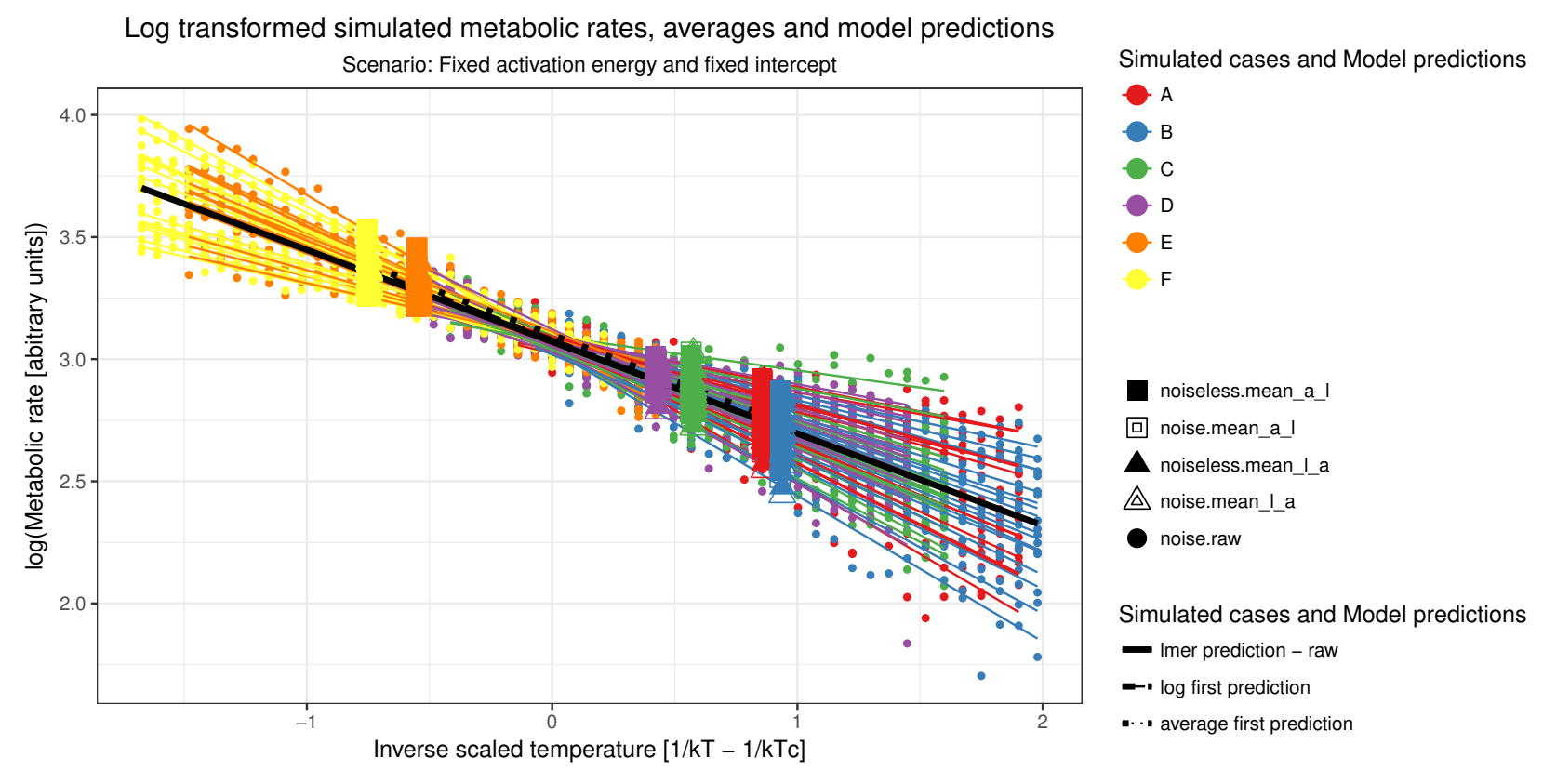

Figure 2: Examplary Arrhenius plot for scenario one

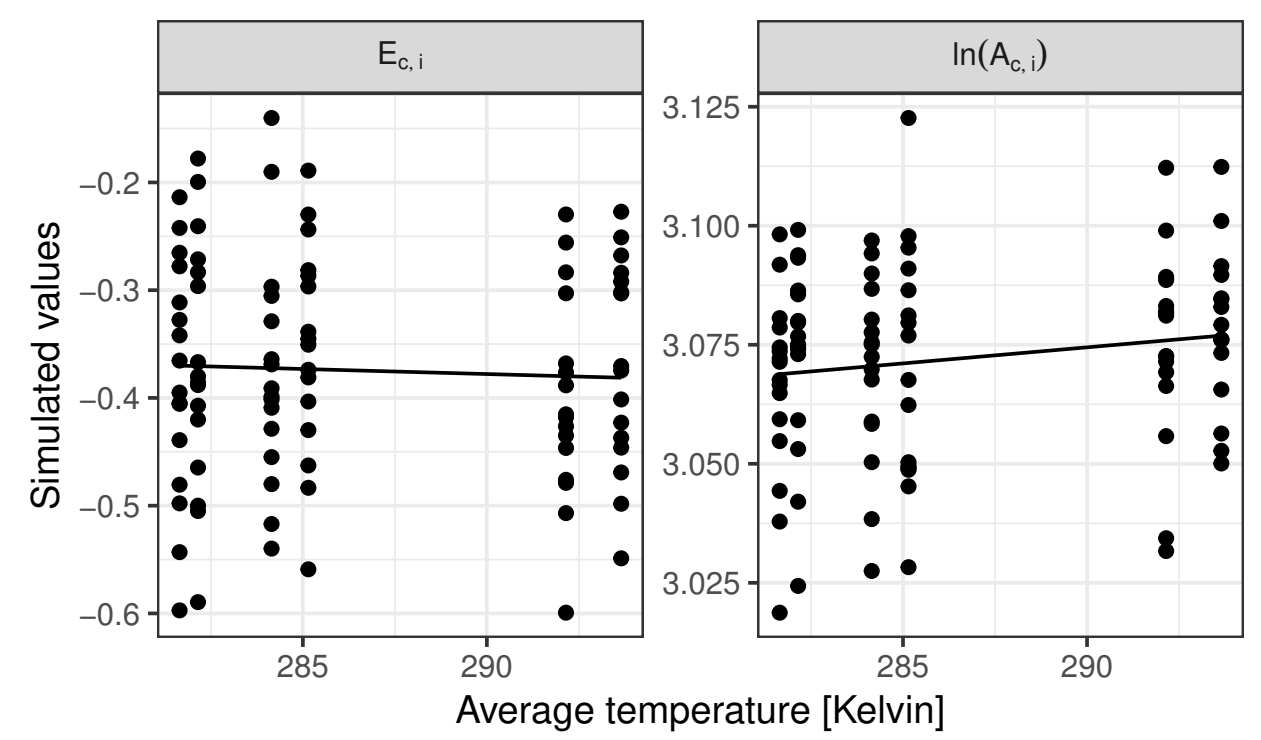

Figure 3: Scenario one: Examplary regression of $\ln A_{c, i}$ and $E_{c, i}$ vs. $\overline{T_{c}}$ 


\subsection{Summary results scenario one:}

In this scenario all three approaches are able to estimate $\overline{\ln A_{c, i}}$ and $\overline{E_{c, i}}$ reasonable well (see figure 4 and 5 ). However, the variance in $\overline{\ln A_{c, i}}-\hat{A}$ is higher for the $\log$-first and average-first approach compared to the LMER-approach. While the log-first approach seems to systematically underestimate $\overline{\ln A_{c, i}}$, the average-first approach seem to systematically overestimate $\overline{\ln A_{c, i}}$ (see figure 4).

The variance in the estimates of $\overline{E_{c, i}}$ is low for all approaches, yet estimates by the LMER-approach and the log-first approach show small systematic overestimation of $\overline{E_{c, i}}$. Overall, for both parameters the LMERapproach has the highest score in zero difference for $\overline{\ln A_{c, i}}-\hat{A}$ and $\overline{E_{c, i}}-\hat{E}$ (see figure 4).

This is inline with very similar density distributions of estimated compared to simulated parameters for all 3 approaches (see figure 5) and the fact that $\hat{A}$ and $\hat{E}$ are almost exclusively determined by $\overline{\ln A_{c, i}}$ and $\overline{E_{c, i}}$ respectively (see tables $3,5,7,9,11$ and 13 , as well as figures 6,7 and 8 ).

\subsection{Scenario one: Comparison between $\overline{E_{c, i}}$ and $\overline{\ln A_{c, i}}$ and $\hat{E}$ and $\hat{A}$ from the 3 different estimation approaches}
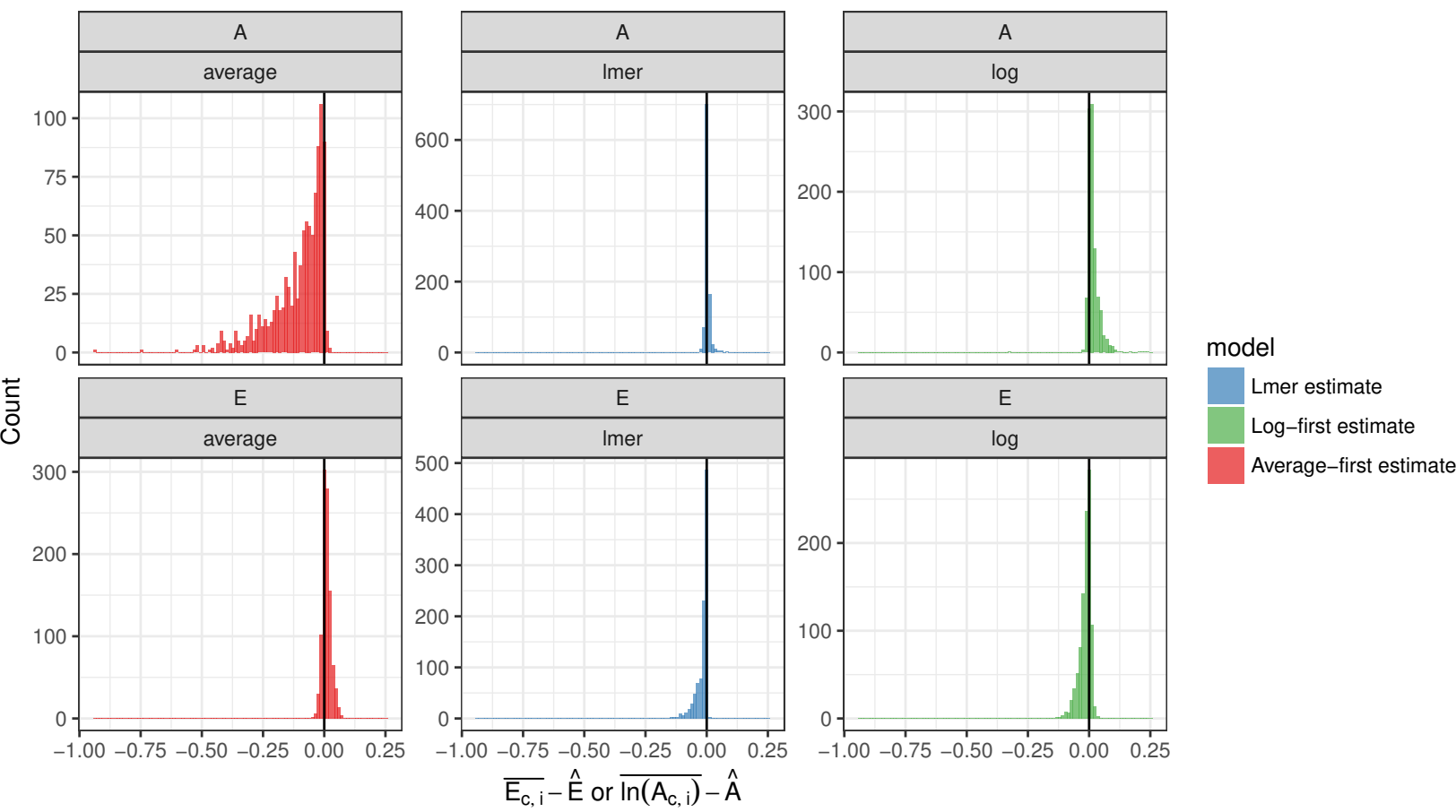

Figure 4: Scenario one: Comparison of $\overline{E_{c, i}}-\hat{E}$ and $\overline{\ln A_{c, i}}-\hat{A}$ for the 3 tested estimation approaches 


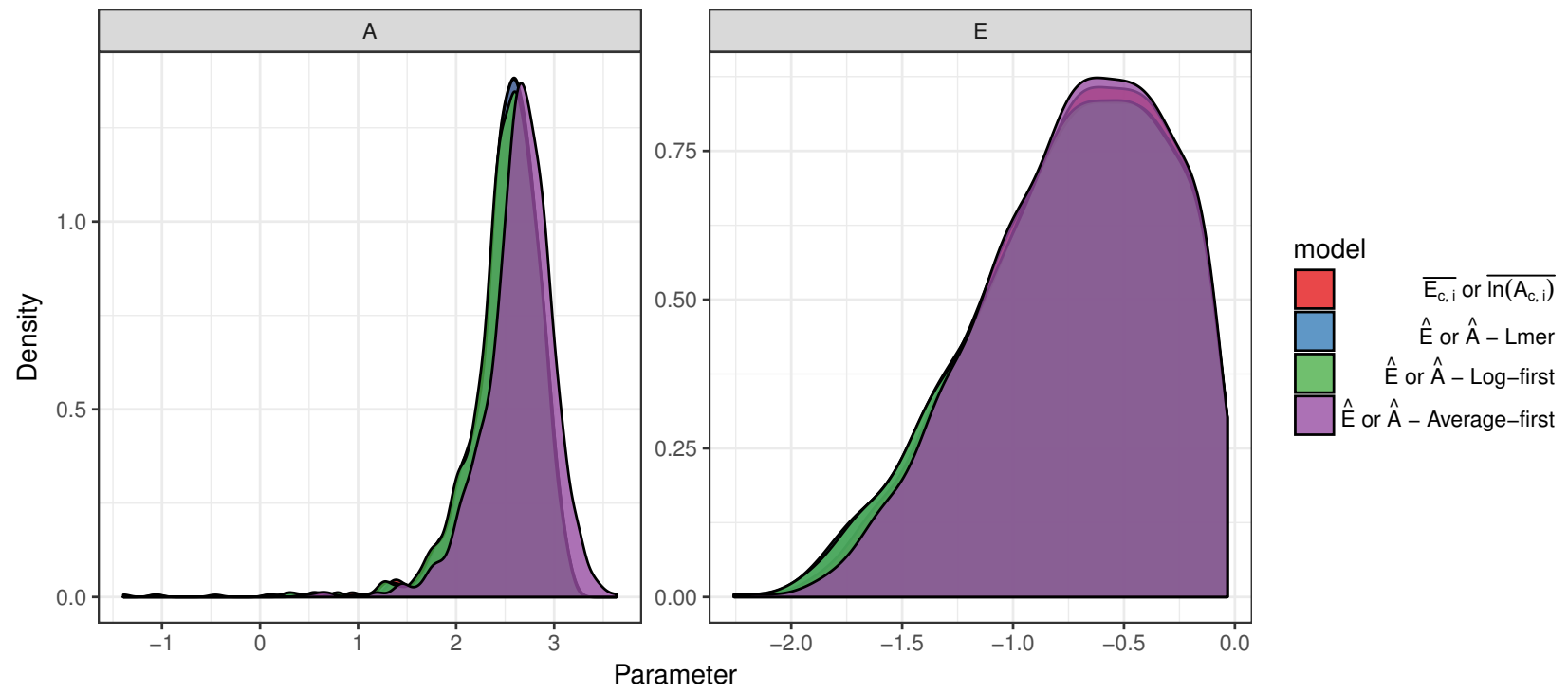

Figure 5: Scenario one: Comparison between simulated and estimated parameter 


\subsection{Scenario one: Detailed results on the influnece of chance correlation of}

$\ln A_{c, i} E_{c, i}$ with $\bar{T}_{c}$ on the estimates of $\hat{E}$ and $\hat{A}$

3.4.1 Log-first approach - Best model to explain $\hat{E}$ and $\hat{A}$ based on $\overline{E_{c, i}}, \overline{\ln A_{c, i}}, \beta_{E_{c, i}}$ and $\beta_{A_{c, i}}$
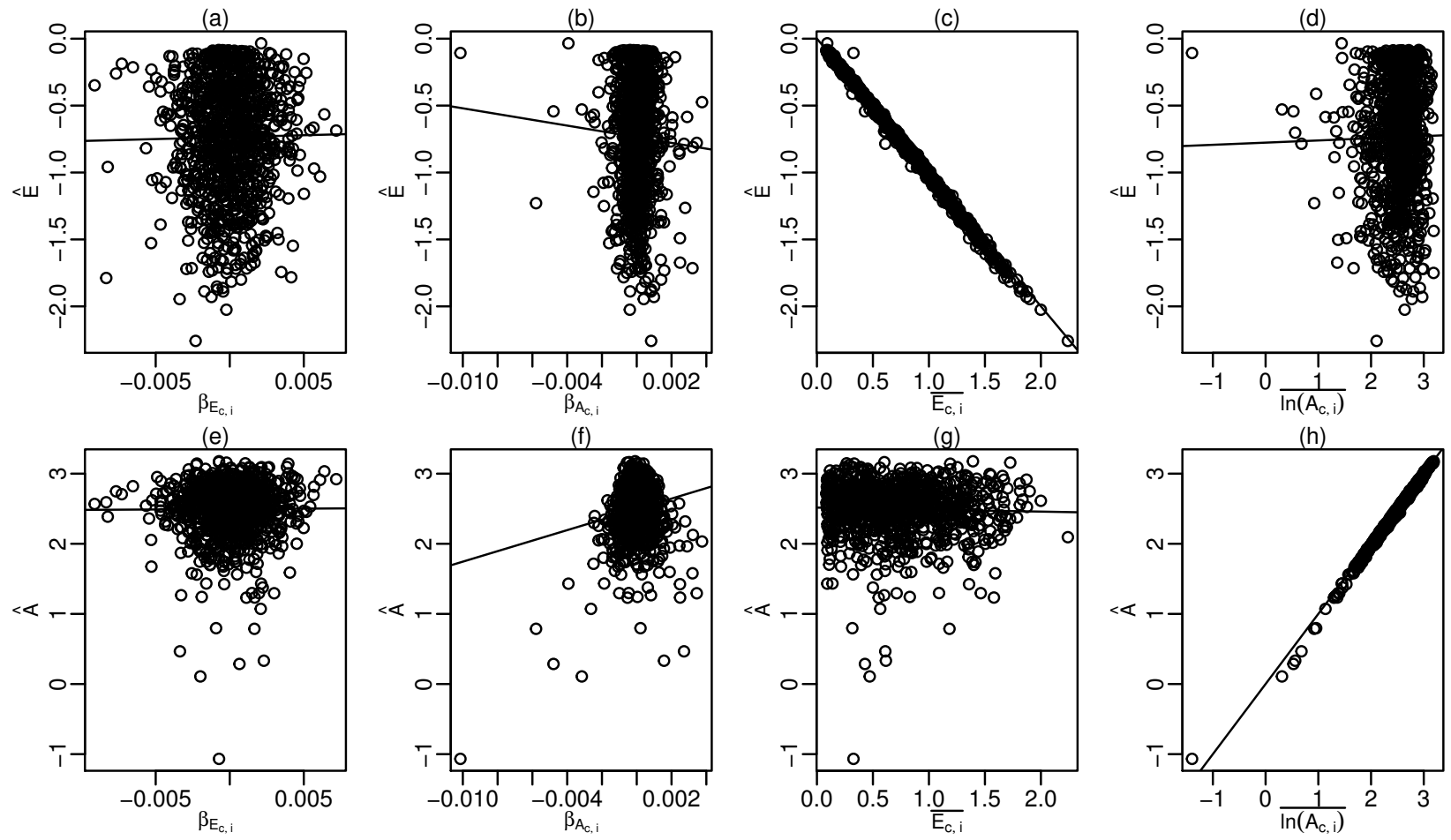

Figure 6: Scenario one - Log-first approach: Influence of $\beta_{E c, i}, \beta_{A c, i}, \overline{E_{c, i}}$, and $\overline{\ln A_{c, i}}$ on $\hat{E}$ and $\hat{A}$. Solid lines depict linear regression relations for all plot exept (c) and (h) were a bisection line is plotted since identity relations are expected.

Table 2: Scenario one - Log-first approach: Best model for $\hat{E}$

\begin{tabular}{lrrrr}
\hline & Estimate & Std. Error & t.value & $\operatorname{Pr}(>|t|)$ \\
\hline (Intercept) & 0.028 & 0.008 & 3.408 & 0.001 \\
$\overline{\ln A_{c, i}}$ & -0.008 & 0.003 & -2.451 & 0.014 \\
$\overline{E_{c, i}}$ & -1.125 & 0.011 & -106.382 & 0.000 \\
$\overline{\ln A_{c, i}}: \overline{E_{c, i}}$ & 0.037 & 0.004 & 8.826 & 0.000 \\
\hline
\end{tabular}

Table 3: Scenario one - Log-first approach: Relative importance of best model parameters to explain $\hat{E}$

\begin{tabular}{lr}
\hline & Rel. Importance \\
\hline$\overline{\ln A_{c, i}}$ & 0 \\
$\overline{E_{c, i}}$ & 1 \\
$\overline{\ln A_{c, i}}: \overline{E_{c, i}}$ & 0 \\
\hline
\end{tabular}


Table 4: Scenario one - Log-first approach: Best model for $\hat{A}$

\begin{tabular}{lrrrr}
\hline & Estimate & Std. Error & t.value & $\operatorname{Pr}(>|t|)$ \\
\hline (Intercept) & 0.000 & 0.009 & -0.034 & 0.973 \\
$\overline{\ln A_{c, i}}$ & 1.002 & 0.004 & 278.490 & 0.000 \\
$\overline{E_{c, i}}$ & -0.124 & 0.012 & -10.633 & 0.000 \\
$\overline{\ln A_{c, i}}: \overline{E_{c, i}}$ & 0.038 & 0.005 & 8.266 & 0.000 \\
\hline
\end{tabular}

Table 5: Scenario one - Log-first approach: Relative importance of best model parameters to explain $\hat{A}$

\begin{tabular}{lr}
\hline & Rel. Importance \\
\hline$\overline{\overline{\ln A_{c, i}}}$ & 0.999 \\
$\overline{E_{c, i}}$ & 0.001 \\
$\overline{\ln A_{c, i}}: \overline{E_{c, i}}$ & 0.000 \\
\hline
\end{tabular}


3.4.2 Average-first approach - Best model to explain $\hat{E}$ and $\hat{A}$ based on $\overline{E_{c, i}}, \overline{\ln A_{c, i}}, \beta_{E_{c, i}}$ and $\beta_{A_{c, i}}$
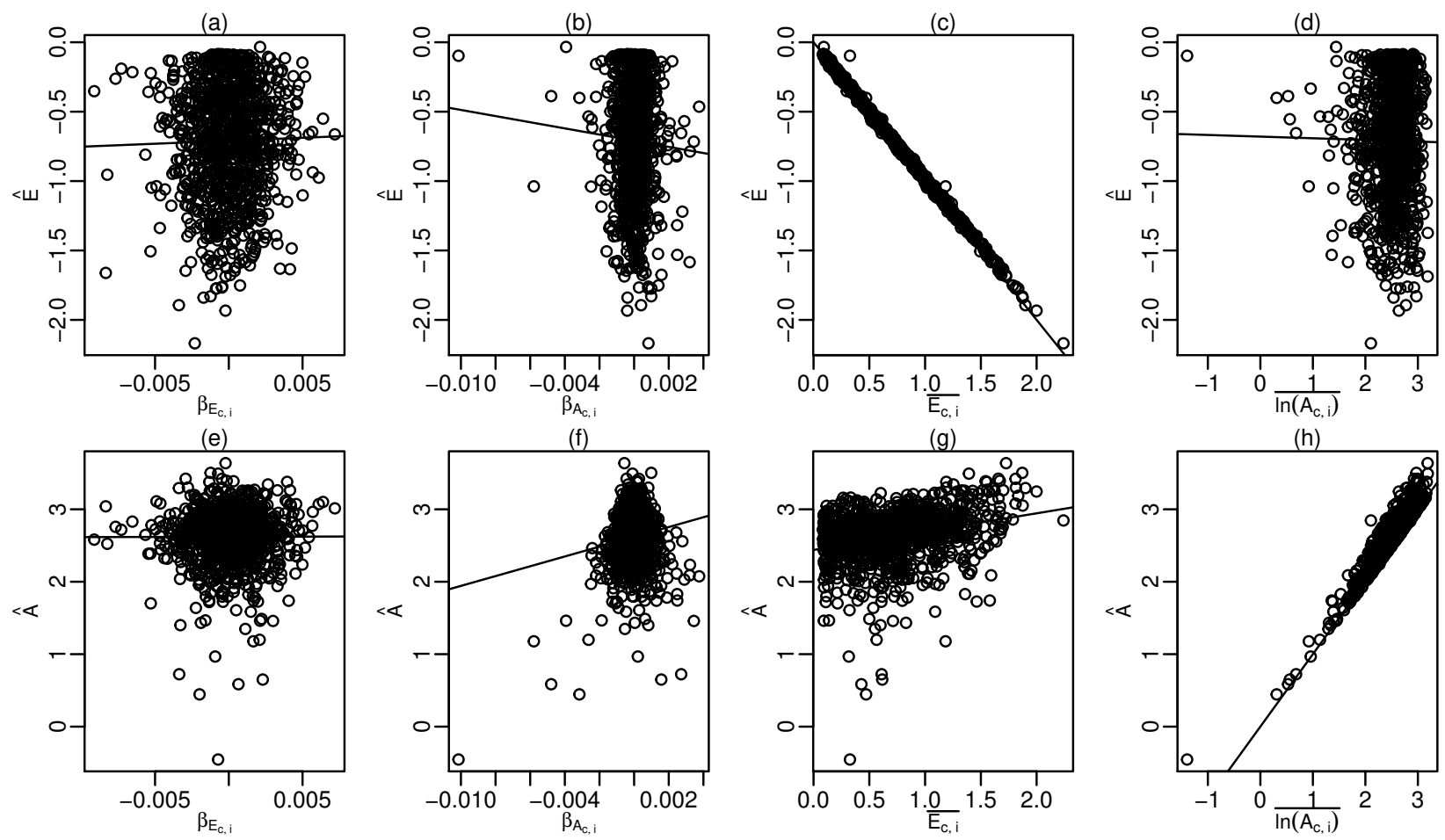

Figure 7: Scenario one - Average-first approach: Influence of $\beta_{E c, i}, \beta_{A c, i}, \overline{E_{c, i}}$, and $\overline{\ln A_{c, i}}$ on $\hat{E}$ and $\hat{A}$. Solid lines depict linear regression relations for all plot exept (c) and (h) were a bisection line is plotted since identity relations are expected.

Table 6: Scenario one - average-first approach: Best model for $\hat{E}$

\begin{tabular}{lrrrr}
\hline & Estimate & Std. Error & t.value & $\operatorname{Pr}(>|t|)$ \\
\hline (Intercept) & 0.033 & 0.007 & 4.893 & 0.000 \\
$\overline{\ln A_{c, i}}$ & -0.016 & 0.003 & -6.105 & 0.000 \\
$\frac{E_{c, i}}{\ln A_{c, i}}: \overline{E_{c, i}}$ & -0.991 & 0.008 & -116.844 & 0.000 \\
\hline
\end{tabular}

Table 7: Scenario one - average-first approach: Relative importance of best model parameters to explain $\hat{E}$

\begin{tabular}{lr}
\hline & Rel. Importance \\
\hline$\overline{\overline{\ln A_{c, i}}}$ & 0 \\
$\overline{E_{c, i}}$ & 1 \\
$\overline{\ln A_{c, i}}: \overline{E_{c, i}}$ & 0 \\
\hline
\end{tabular}


Table 8: Scenario one - average-first approach: Best model for $\hat{A}$

\begin{tabular}{lrrrr}
\hline & Estimate & Std. Error & t.value & $\operatorname{Pr}(>|t|)$ \\
\hline (Intercept) & 0.077 & 0.017 & 4.500 & 0 \\
$\overline{\ln A_{c, i}}$ & 0.940 & 0.007 & 139.700 & 0 \\
$\overline{E_{c, i}}$ & 0.134 & 0.022 & 6.118 & 0 \\
$\overline{\ln A_{c, i}}: \overline{E_{c, i}}$ & 0.047 & 0.009 & 5.482 & 0 \\
\hline
\end{tabular}

Table 9: Scenario one - average-first approach: Relative importance of best model parameters to explain $\hat{A}$

\begin{tabular}{lr}
\hline & Rel. Importance \\
\hline$\overline{\ln A_{c, i}}$ & 0.921 \\
$\overline{E_{c, i}}$ & 0.079 \\
$\overline{\ln A_{c, i}}: \overline{E_{c, i}}$ & 0.000 \\
\hline
\end{tabular}


3.4.3 LMER approach - Best model to explain $\hat{E}$ and $\hat{A}$ based on $\overline{E_{c, i}}, \overline{\ln A_{c, i}}, \beta_{E_{c, i}}$ and $\beta_{A_{c, i}}$
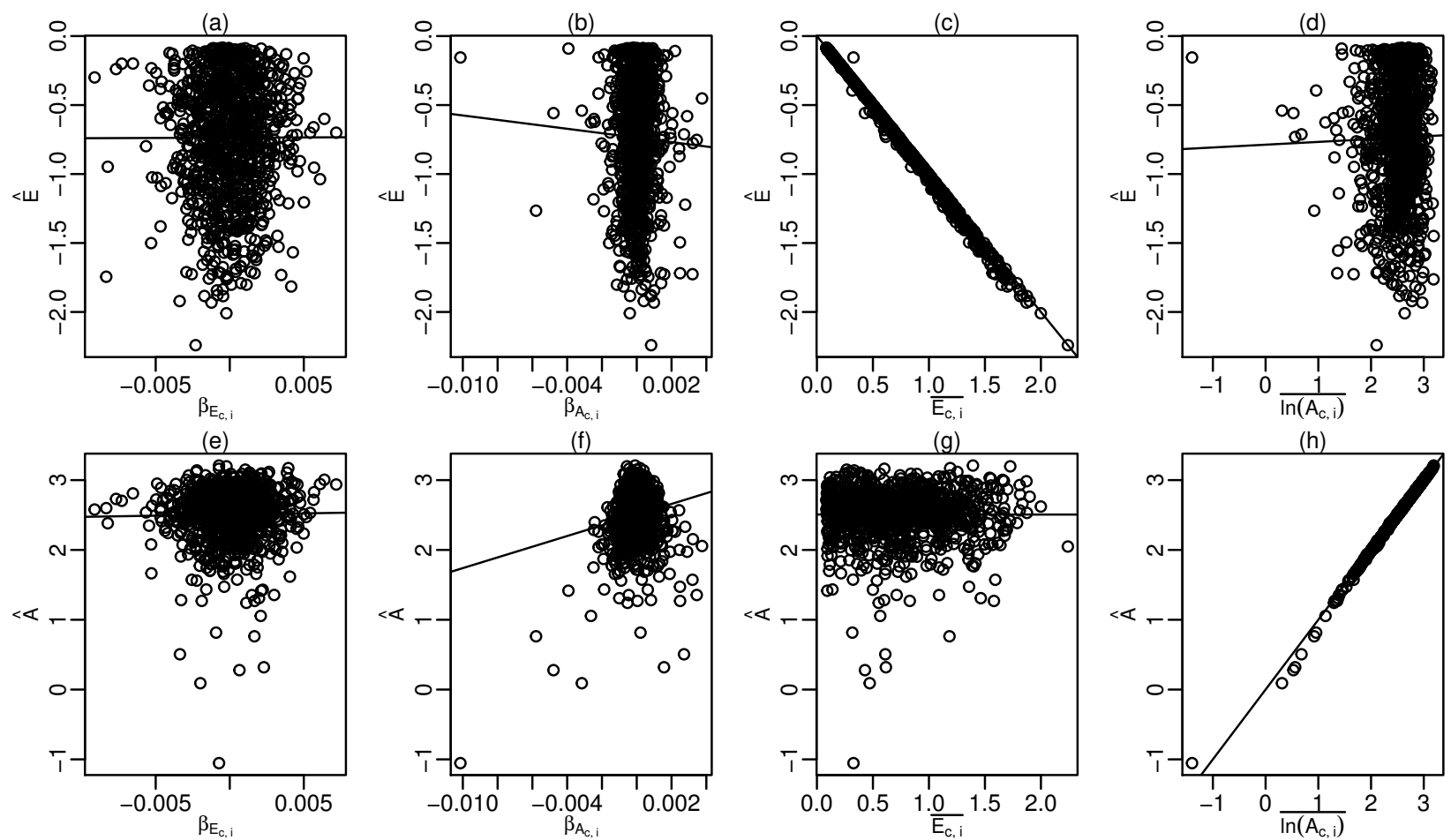

Figure 8: Scenario one - LMER approach: Influence of $\beta_{E c, i}, \beta_{A c, i}, \overline{E_{c, i}}$, and $\overline{\ln A_{c, i}}$ on $\hat{E}$ and $\hat{A}$. Solid lines depict linear regression relations for all plot exept (c) and (h) were a bisection line is plotted since identity relations are expected.

Table 10: Scenario one - lmer approach: Best model for $\hat{E}$

\begin{tabular}{lrrrr}
\hline & Estimate & Std. Error & t.value & $\operatorname{Pr}(>|t|)$ \\
\hline (Intercept) & 0.023 & 0.007 & 3.116 & 0.002 \\
$\overline{\ln A_{c, i}}$ & -0.005 & 0.003 & -1.759 & 0.079 \\
$\overline{E_{c, i}}$ & -1.133 & 0.009 & -122.179 & 0.000 \\
$\overline{\ln A_{c, i}}: \overline{E_{c, i}}$ & 0.038 & 0.004 & 10.474 & 0.000 \\
\hline
\end{tabular}

Table 11: Scenario one - lmer approach: Relative importance of best model parameters to explain $\hat{E}$

\begin{tabular}{lr}
\hline & Rel. Importance \\
\hline$\overline{\overline{\ln A_{c, i}}}$ & 0 \\
$\overline{E_{c, i}}$ & 1 \\
$\overline{\ln A_{c, i}}: \overline{E_{c, i}}$ & 0 \\
\hline
\end{tabular}


Table 12: Scenario one - lmer approach: Best model for $\hat{A}$

\begin{tabular}{lrrrr}
\hline & Estimate & Std. Error & t.value & $\operatorname{Pr}(>|t|)$ \\
\hline (Intercept) & 0.005 & 0.008 & 0.669 & 0.504 \\
$\overline{\ln A_{c, i}}$ & 0.996 & 0.003 & 314.838 & 0.000 \\
$\overline{E_{c, i}}$ & -0.090 & 0.010 & -8.776 & 0.000 \\
$\overline{\ln A_{c, i}}: \overline{E_{c, i}}$ & 0.036 & 0.004 & 8.812 & 0.000 \\
\hline
\end{tabular}

Table 13: Scenario one - lmer approach: Relative importance of best model parameters to explain $\hat{A}$

\begin{tabular}{lr}
\hline & Rel. Importance \\
\hline$\overline{\ln A_{c, i}}$ & 1 \\
$\overline{E_{c, i}}$ & 0 \\
$\overline{\ln A_{c, i}}: \overline{E_{c, i}}$ & 0 \\
\hline
\end{tabular}




\section{Scenario two: All entities have the same generic activation en- ergy $E_{c}$ but have a variable intercept $A_{c}$}

\subsection{Description and assumptions of scenario two}

In this scenario it is assumed that there exists one generic temperature dependence $E_{c}=E \forall c$, however $A_{c}$ is assumed to be different between entities. This makes scenario two more realistic and at the same time accords to MTE. Since $A_{c}$ differ between entities, $\overline{\ln A_{c, i}}$ might exhibit chance correlation with $\bar{T}_{c}$ (see figure 9 ).

Simulated metabolic rates and respective averages

Scenario: Fixed activation energy and variable intercept

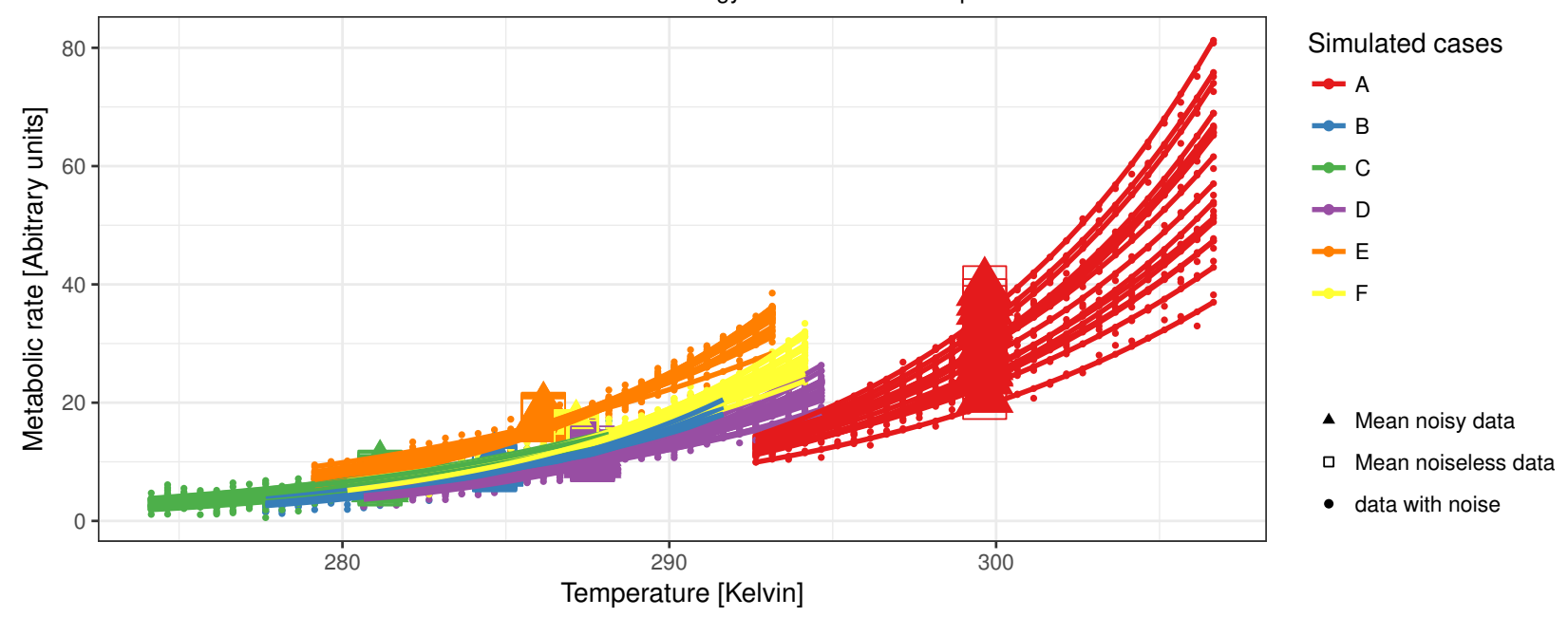

Log transformed simulated metabolic rates, averages and model predictions

Scenario: Fixed activation energy and variable intercept

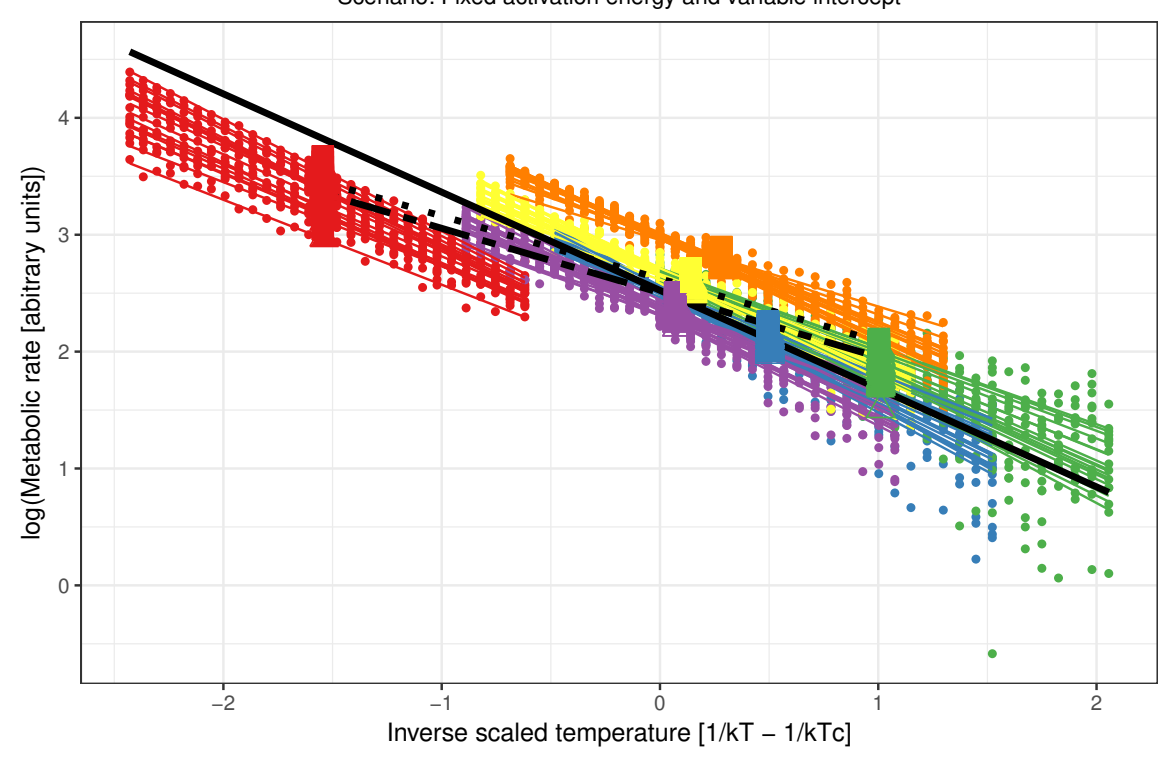

Simulated cases and Model predictions

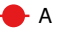

$B$

C

- E

F

noiseless.mean_a_l

回 noise.mean_a_l

$\Delta$ noiseless.mean__aa

$\triangle$ noise.mean_l_a

- noise.raw

Simulated cases and Model predictions

- Imer prediction - raw

- $\log$ first prediction

.... average first prediction 


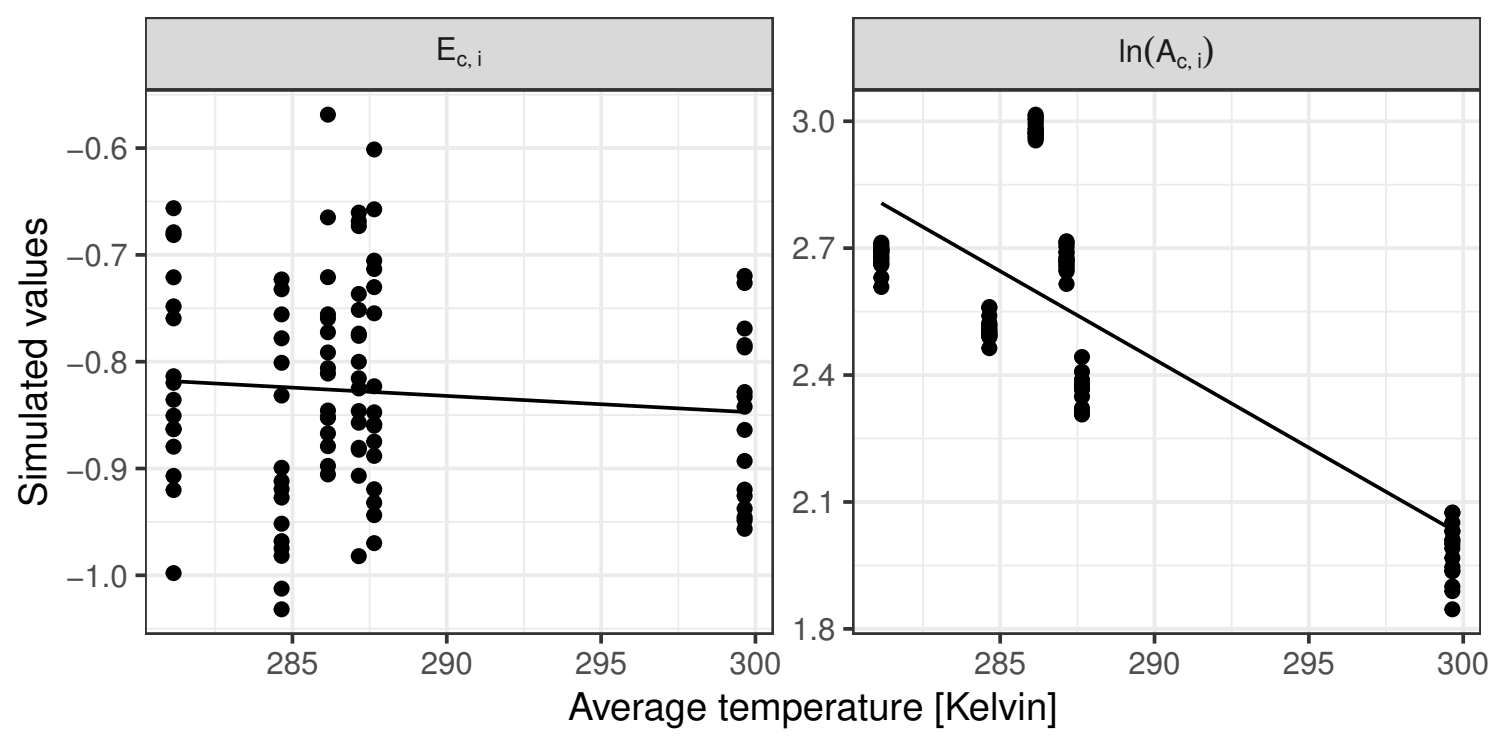

Figure 9: Scenario two: Regression of $\ln A_{c, i}$ and $E_{c, i}$ vs. $\overline{T_{c}}$ 


\subsection{Summary results scenario two:}

$\hat{E}$ for both the average-first and log-first approaches is mainly determined by $\overline{E_{c, i}}$ but also to a considerable amount by $\beta_{A_{c, i}}$, the chance co variation of simulated $\overline{\ln A_{c, i}}$ values with $\bar{T}_{c}$ (see tables 15,19 and figures 12 , 13). This is not the case for the LMER approach (see table 23 and figure 14).

Similar, $\hat{A}$ is not only determined by $\overline{\ln A_{c, i}}$ but also to a considerable degree by $\beta_{A_{c, i}}$ and partly also $\overline{E_{c, i}}$ for the average-first and log-first approach (see tables 17, 21 and figures 12, 13). Again, this is not the case for the LMER approach were estimated $\hat{A}$ are only driven by $\overline{\ln A_{c, i}}$ (see table 25 and figure 14).

This is consistent with the fact that both average based approaches are marked by a large spread in $\overline{\ln A_{c, i}}-\hat{A}$ and $\overline{E_{c, i}}-\hat{E}$ (see figure 10), indicating that while $\hat{A}$ and $\hat{E}$ might be close to $\bar{E}$ and $\bar{A}$ also large deviations are possible likely driven by $\beta_{A_{c, i}}$. In contrast difference between estimated and simulated values have a much smaller spread for the LMER approach. Similar, the density distributions of the estimated parameters from the LMER approach are much closer to the density distributions of the simulated parameters, as is the case for the ones from the average based approaches (see figure 11).

\subsection{Scenario two: Comparison between $\overline{E_{c, i}}$ and $\overline{\ln A_{c, i}}$ and $\hat{E}$ and $\hat{A}$ from the 3 different estimation approaches}
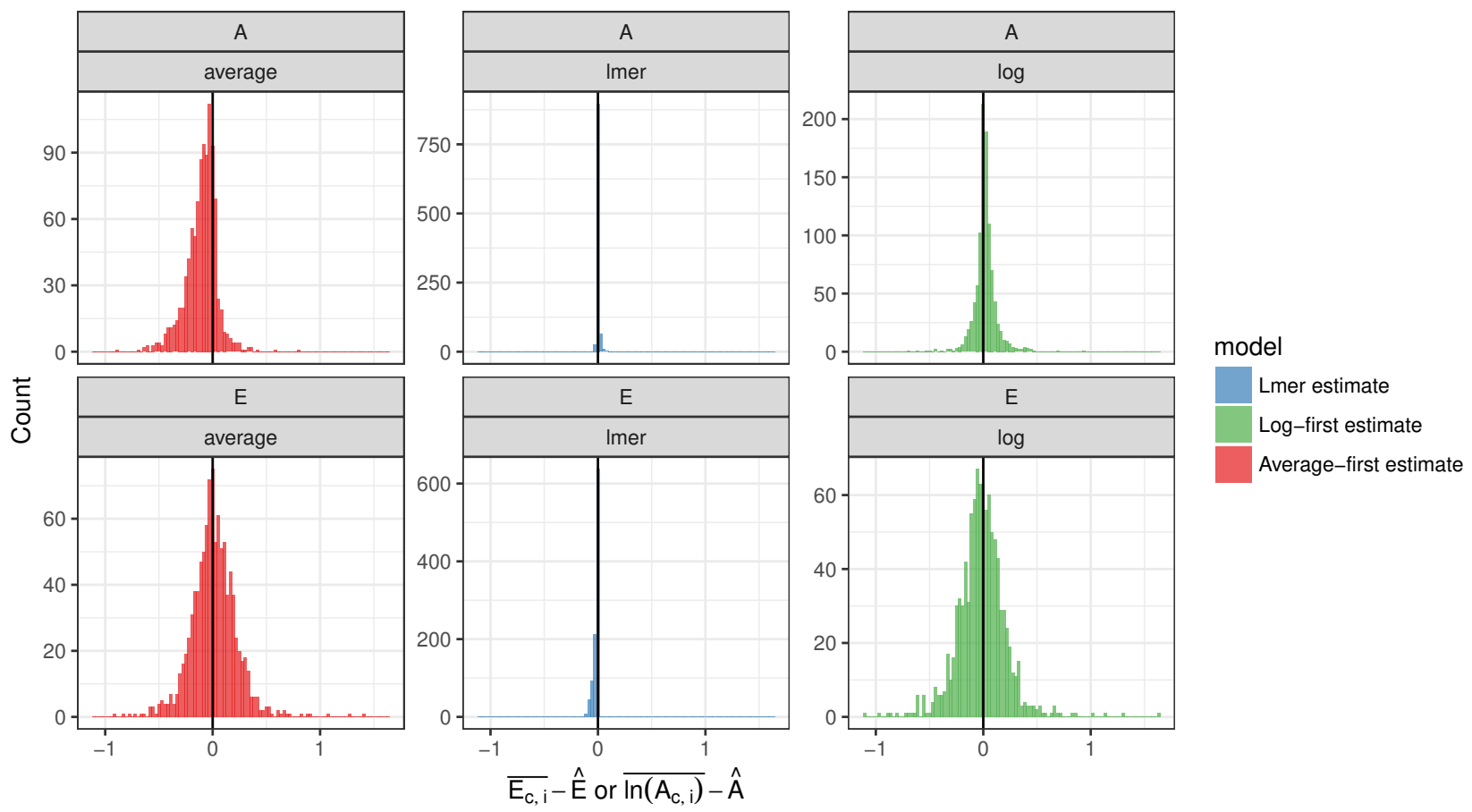

Figure 10: Scenario two: Comparison of $\overline{E_{c, i}}-\hat{E}$ and $\overline{\ln A_{c, i}}-\hat{A}$ for the 3 tested estimation approaches 

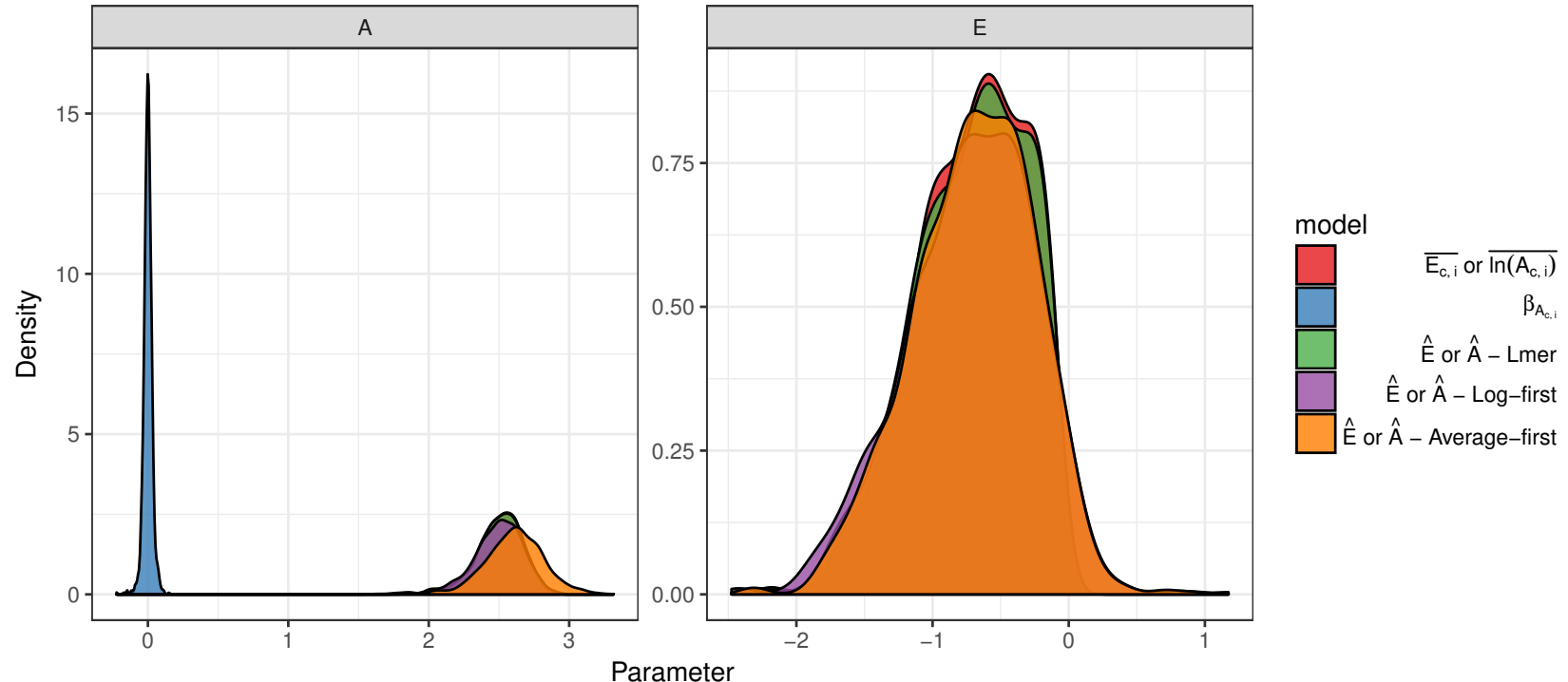

Figure 11: Scenario two: Comparison between simulated and estimated parameter 


\subsection{Scenario two: Detailed results on the influnece of chance correlation of}

$\ln A_{c, i} E_{c, i}$ with $\bar{T}_{c}$ on the estimates of $\hat{E}$ and $\hat{A}$

4.4.1 Log-first approach - Best model to explain $\hat{E}$ and $\hat{A}$ based on $\overline{E_{c, i}}, \overline{\ln A_{c, i}}, \beta_{E_{c, i}}$ and $\beta_{A_{c, i}}$
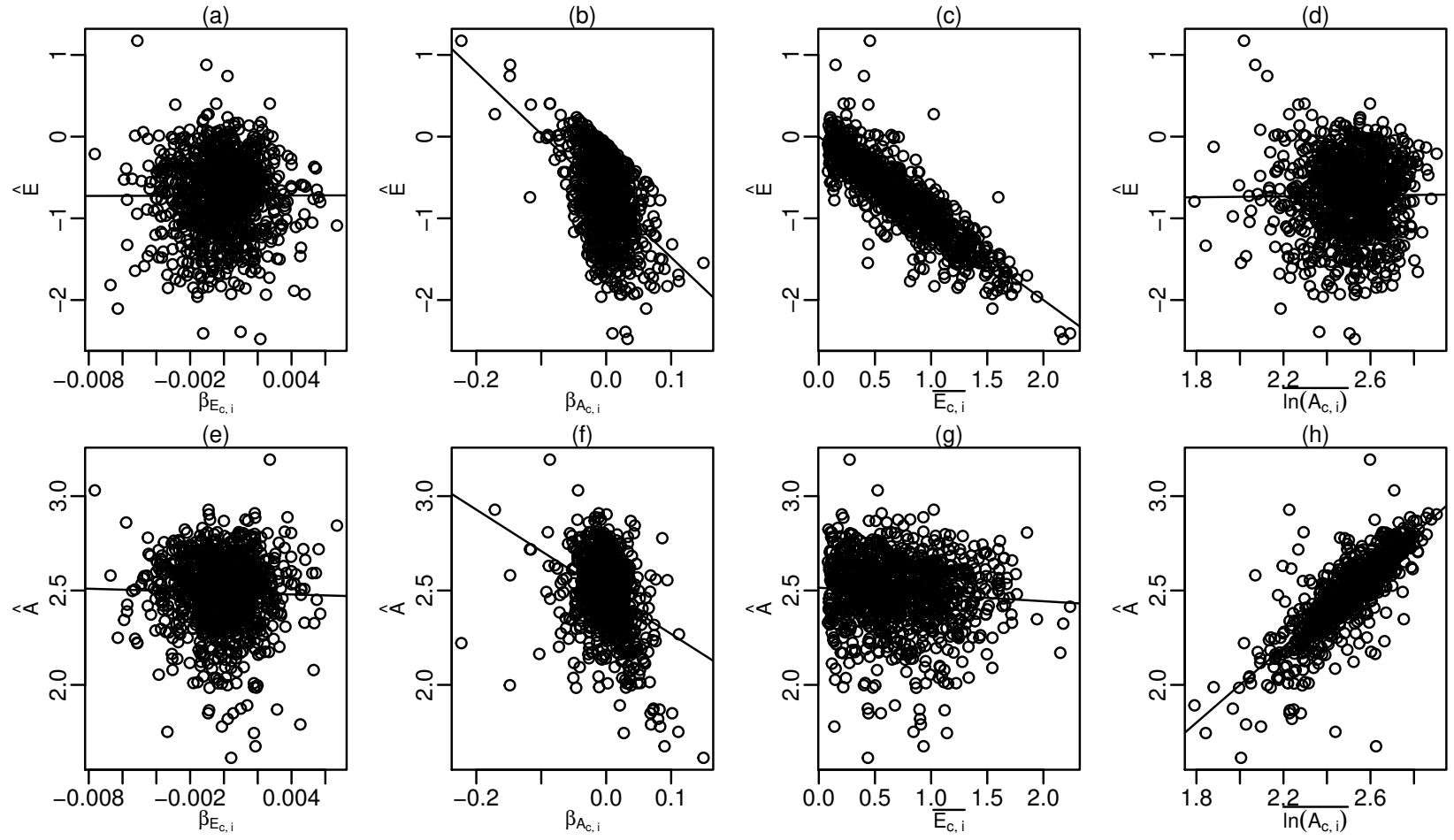

Figure 12: Scenario two - Log-first approach: Influence of $\beta_{E c, i}, \beta_{A c, i}, \overline{E_{c, i}}$, and $\overline{\ln A_{c, i}}$ on $\hat{E}$ and $\hat{A}$. Solid lines depict linear regression relations for all plot exept (c) and (h) were a bisection line is plotted since identity relations are expected.

Table 14: Scenario two - Log-first approach: Best model for $\hat{E}$

\begin{tabular}{lrrrr}
\hline & Estimate & Std. Error & t.value & $\operatorname{Pr}(>|t|)$ \\
\hline (Intercept) & 0.011 & 0.019 & 0.571 & 0.568 \\
$\ln A_{c, i}$ & -0.001 & 0.008 & -0.065 & 0.948 \\
$\beta_{A_{c, i}}$ & -7.705 & 0.229 & -33.684 & 0.000 \\
$\overline{E_{c, i}}$ & -1.117 & 0.025 & -44.764 & 0.000 \\
$\overline{\ln A_{c, i}}: \beta_{A_{c, i}}$ & 0.171 & 0.097 & 1.767 & 0.078 \\
$\overline{\ln A_{c, i}}: \bar{E}_{c, i}$ & 0.032 & 0.010 & 3.213 & 0.001 \\
$\beta_{A_{c, i}}: \overline{E_{c, i}}$ & -0.300 & 0.053 & -5.712 & 0.000 \\
\hline
\end{tabular}

Table 15: Scenario two - Log-first approach: Relative importance of best model parameters to explain $\hat{E}$

\begin{tabular}{lr}
\hline & Rel. Importance \\
\hline$\overline{\ln A_{c, i}}$ & 0.000 \\
$\beta_{A_{c, i}}$ & 0.233 \\
$\overline{E_{c, i}}$ & 0.767
\end{tabular}




\begin{tabular}{lr}
\hline & Rel. Importance \\
\hline$\overline{\ln A_{c, i}}: \beta_{A_{c, i}}$ & 0.000 \\
$\overline{\ln A_{c, i}}: \overline{E_{c, i}}$ & 0.000 \\
$\beta_{A_{c, i}}: \overline{E_{c, i}}$ & 0.000 \\
\hline
\end{tabular}

Table 16: Scenario two - Log-first approach: Best model for $\hat{A}$

\begin{tabular}{lrrrr}
\hline & Estimate & Std. Error & t.value & $\operatorname{Pr}(>|t|)$ \\
\hline (Intercept) & 0.013 & 0.043 & 0.309 & 0.758 \\
$\ln A_{c, i}$ & 0.996 & 0.017 & 59.315 & 0.000 \\
$\beta_{A_{c, i}}$ & -2.317 & 0.087 & -26.739 & 0.000 \\
\hline$E_{c, i}$ & -0.029 & 0.007 & -4.422 & 0.000 \\
\hline
\end{tabular}

Table 17: Scenario two - Log-first approach: Relative importance of best model parameters to explain $\hat{A}$

\begin{tabular}{lr}
\hline & Rel. Importance \\
\hline$\overline{\ln A_{c, i}}$ & 0.832 \\
$\beta_{A_{c, i}}$ & 0.162 \\
$\overline{E_{c, i}}$ & 0.006 \\
\hline
\end{tabular}


4.4.2 Average-first approach - Best model to explain $\hat{E}$ and $\hat{A}$ based on $\overline{E_{c, i}}, \overline{\ln A_{c, i}}, \beta_{E_{c, i}}$ and $\beta_{A_{c, i}}$
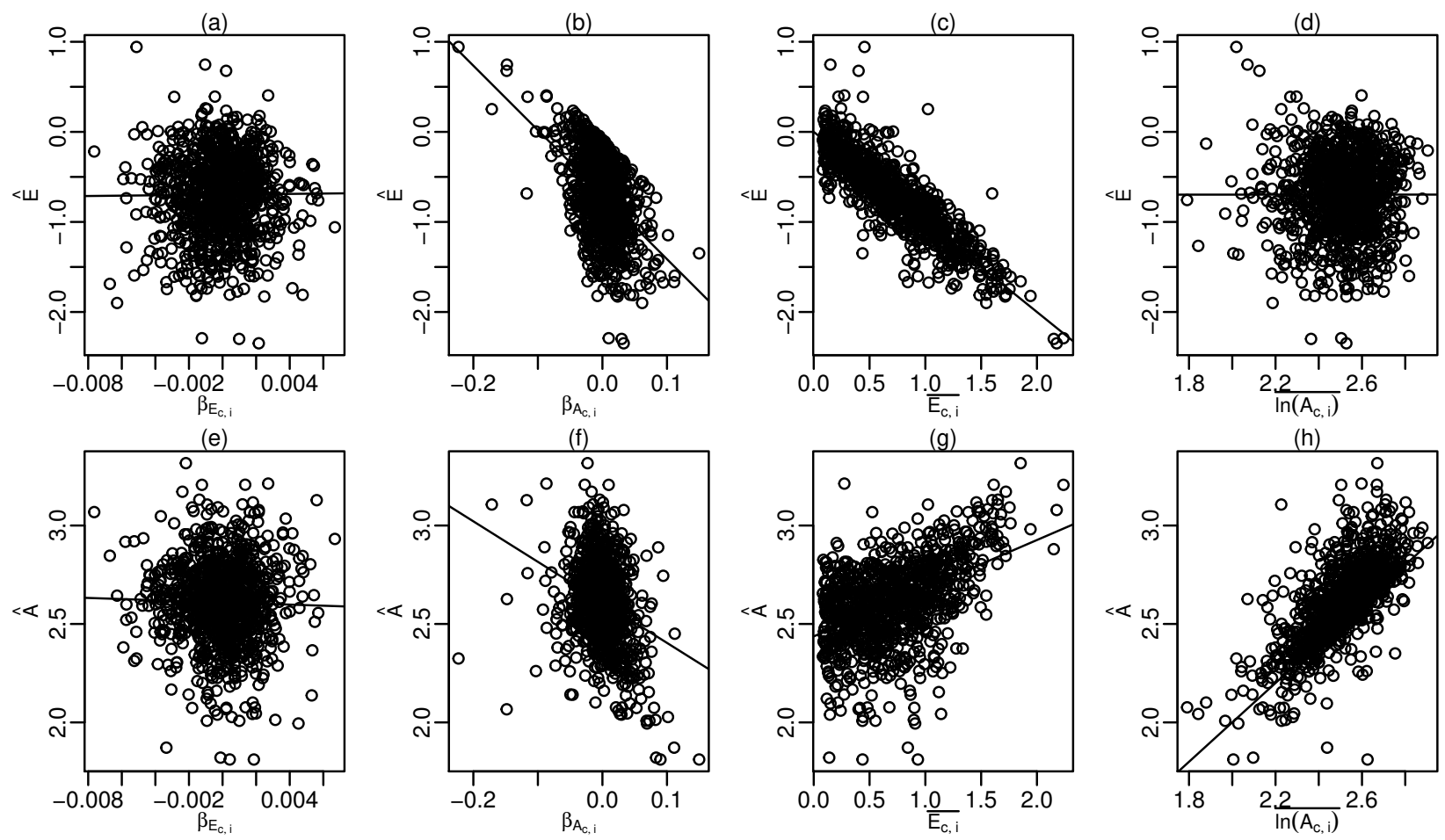

Figure 13: Scenario two - Average-first approach: Influence of $\beta_{E c, i}, \beta_{A c, i}, \overline{E_{c, i}}$, and $\overline{\ln A_{c, i}}$ on $\hat{E}$ and $\hat{A}$. Solid lines depict linear regression relations for all plot exept (c) and (h) were a bisection line is plotted since identity relations are expected.

Table 18: Scenario two - average-first approach: Best model for $\hat{E}$

\begin{tabular}{lrrrr}
\hline & Estimate & Std. Error & t.value & $\operatorname{Pr}(>|t|)$ \\
\hline (Intercept) & -0.033 & 0.015 & -2.191 & 0.029 \\
$\overline{\ln A_{c, i}}$ & 0.010 & 0.006 & 1.709 & 0.088 \\
$\beta_{A_{c, i}}$ & -4.430 & 0.177 & -25.016 & 0.000 \\
$\overline{E_{c, i}}$ & -0.918 & 0.019 & -47.517 & 0.000 \\
$\overline{\ln A_{c, i}}: \beta_{A_{c, i}}$ & -1.074 & 0.075 & -14.319 & 0.000 \\
$\overline{\ln A_{c, i}}: \overline{E_{c, i}}$ & -0.023 & 0.008 & -3.045 & 0.002 \\
$\beta_{A_{c, i}}: \bar{E}_{c, i}$ & -0.178 & 0.041 & -4.382 & 0.000 \\
\hline
\end{tabular}

Table 19: Scenario two - average-first approach: Relative importance of best model parameters to explain $\hat{E}$

\begin{tabular}{lr}
\hline & Rel. Importance \\
\hline$\overline{\ln A_{c, i}}$ & 0.000 \\
$\beta_{A_{c, i}}$ & 0.235 \\
$\overline{E_{c, i}}$ & 0.765 \\
$\overline{\ln A_{c, i}}: \beta_{A_{c, i}}$ & 0.000 \\
$\overline{\ln A_{c, i}}: \overline{E_{c, i}}$ & 0.000
\end{tabular}




\begin{tabular}{lr}
\hline & Rel. Importance \\
\hline$\beta_{A_{c, i}}: \overline{E_{c, i}}$ & 0.000 \\
\hline
\end{tabular}

Table 20: Scenario two - average-first approach: Best model for $\hat{A}$

\begin{tabular}{lrrrr}
\hline & Estimate & Std. Error & t.value & $\operatorname{Pr}(>|t|)$ \\
\hline (Intercept) & 0.028 & 0.044 & 0.647 & 0.518 \\
$\overline{\ln A_{c, i}}$ & 0.959 & 0.017 & 55.244 & 0.000 \\
$\beta_{A_{c, i}}$ & -0.558 & 1.043 & -0.535 & 0.593 \\
$\overline{E_{c, i}}$ & 0.251 & 0.007 & 36.915 & 0.000 \\
$\overline{\ln A_{c, i}}: \beta_{A_{c, i}}$ & -0.685 & 0.436 & -1.570 & 0.117 \\
\hline
\end{tabular}

Table 21: Scenario two - average-first approach: Relative importance of best model parameters to explain $\hat{A}$

\begin{tabular}{lr}
\hline & Rel. Importance \\
\hline$\overline{\ln A_{c, i}}$ & 0.610 \\
$\beta_{A_{c, i}}$ & 0.115 \\
$\overline{E_{c, i}}$ & 0.274 \\
$\overline{\ln A_{c, i}}: \beta_{A_{c, i}}$ & 0.001 \\
\hline
\end{tabular}


4.4.3 LMER approach - Best model to explain $\hat{E}$ and $\hat{A}$ based on $\overline{E_{c, i}}, \overline{\ln A_{c, i}}, \beta_{E_{c, i}}$ and $\beta_{A_{c, i}}$
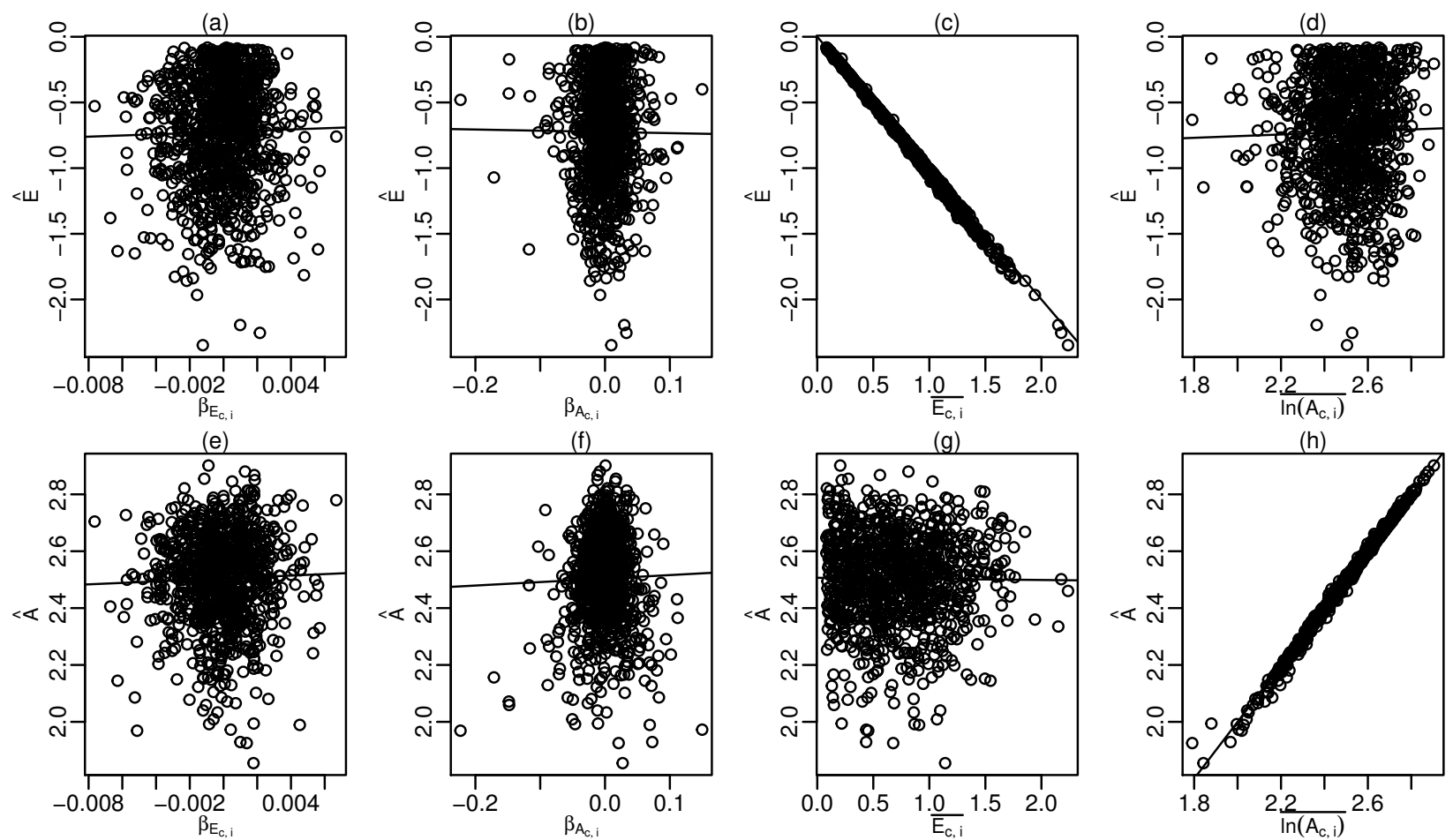

Figure 14: Scenario two - LMER approach: Influence of $\beta_{E c, i}, \beta_{A c, i}, \overline{E_{c, i}}$, and $\overline{\ln A_{c, i}}$ on $\hat{E}$ and $\hat{A}$. Solid lines depict linear regression relations for all plot exept (c) and (h) were a bisection line is plotted since identity relations are expected.

Table 22: Scenario two - lmer approach: Best model for $\hat{E}$

\begin{tabular}{lrrrr}
\hline & Estimate & Std. Error & t.value & $\operatorname{Pr}(>|t|)$ \\
\hline (Intercept) & 0.019 & 0.016 & 1.180 & 0.238 \\
$\overline{\ln A_{c, i}}$ & -0.003 & 0.006 & -0.475 & 0.635 \\
$\beta_{A_{c, i}}$ & 0.747 & 0.193 & 3.881 & 0.000 \\
$\overline{E_{c, i}}$ & -1.135 & 0.021 & -54.084 & 0.000 \\
$\overline{\ln A_{c, i}}: \beta_{A_{c, i}}$ & -0.304 & 0.082 & -3.722 & 0.000 \\
$\ln A_{c, i}: \bar{E}_{c, i}$ & 0.037 & 0.008 & 4.501 & 0.000 \\
$\beta_{A_{c, i}}: \overline{E_{c, i}}$ & -0.090 & 0.044 & -2.034 & 0.042 \\
\hline
\end{tabular}

Table 23: Scenario two - lmer approach: Relative importance of best model parameters to explain $\hat{E}$

\begin{tabular}{lr}
\hline & Rel. Importance \\
$\overline{\overline{\ln A_{c, i}}}$ & 0 \\
$\frac{\beta_{A_{c, i}}}{\overline{E_{c, i}}}: \beta_{A_{c, i}}$ & 0 \\
$\overline{\ln A_{c, i}}: \overline{E_{c, i}}$ & 1 \\
$\overline{\ln A_{c, i}}: \overline{E_{c, i}}$ & 0 \\
$\beta_{A_{c, i}}$ & 0 \\
& 0
\end{tabular}




$\overline{\text { Rel. Importance }}$

Table 24: Scenario two - lmer approach: Best model for $\hat{A}$

\begin{tabular}{lrrrr}
\hline & Estimate & Std. Error & t.value & $\operatorname{Pr}(>|t|)$ \\
\hline (Intercept) & -0.009 & 0.011 & -0.850 & 0.395 \\
$\overline{\ln A_{c, i}}$ & 1.002 & 0.004 & 230.270 & 0.000 \\
$\beta_{A_{c, i}}$ & 0.341 & 0.129 & 2.646 & 0.008 \\
$\overline{E_{c, i}}$ & -0.051 & 0.014 & -3.615 & 0.000 \\
$\overline{\ln A_{c, i}}: \beta_{A_{c, i}}$ & -0.110 & 0.055 & -2.006 & 0.045 \\
$\overline{\ln A_{c, i}}: \overline{E_{c, i}}$ & 0.021 & 0.006 & 3.782 & 0.000 \\
$\beta_{A_{c, i}}: \overline{E_{c, i}}$ & -0.119 & 0.030 & -4.007 & 0.000 \\
\hline
\end{tabular}

Table 25: Scenario two - lmer approach: Relative importance of best model parameters to explain $\hat{A}$

\begin{tabular}{lr}
\hline & Rel. Importance \\
$\overline{\overline{\ln A_{c, i}}}$ & 0.999 \\
$\beta_{A_{c, i}}$ & 0.000 \\
$\overline{E_{c, i}}$ & 0.000 \\
$\overline{\ln A_{c, i}}: \beta_{A_{c, i}}$ & 0.000 \\
$\overline{\ln A_{c, i}}: \overline{E_{c, i}}$ & 0.000 \\
$\beta_{A_{c, i}}: \overline{E_{c, i}}$ & 0.000 \\
\hline
\end{tabular}




\section{Scenario three: All entities have the same generic absolute metabolic rate $A_{c}$ but variable $E_{c}$}

\subsection{Description and assumptions of scenario three}

In this scenario it is assumed that $E_{c}$ can vary with entity, but independent of $\bar{T}_{c}$. For the absolute metabolic rates it is assumed that $A_{c}=A \forall c$, i.e. similar production levels for all entities are simulated. This scenario no longer assumes an unchangeable generic activation energy as proposed by MTE but allows random variation of $E_{c}$. Chance correlation of $E_{c}$ with $\bar{T}_{c}$ can occur, particular since, like in the experiment, temperature ranges are represented by one entity only (see figure 15).
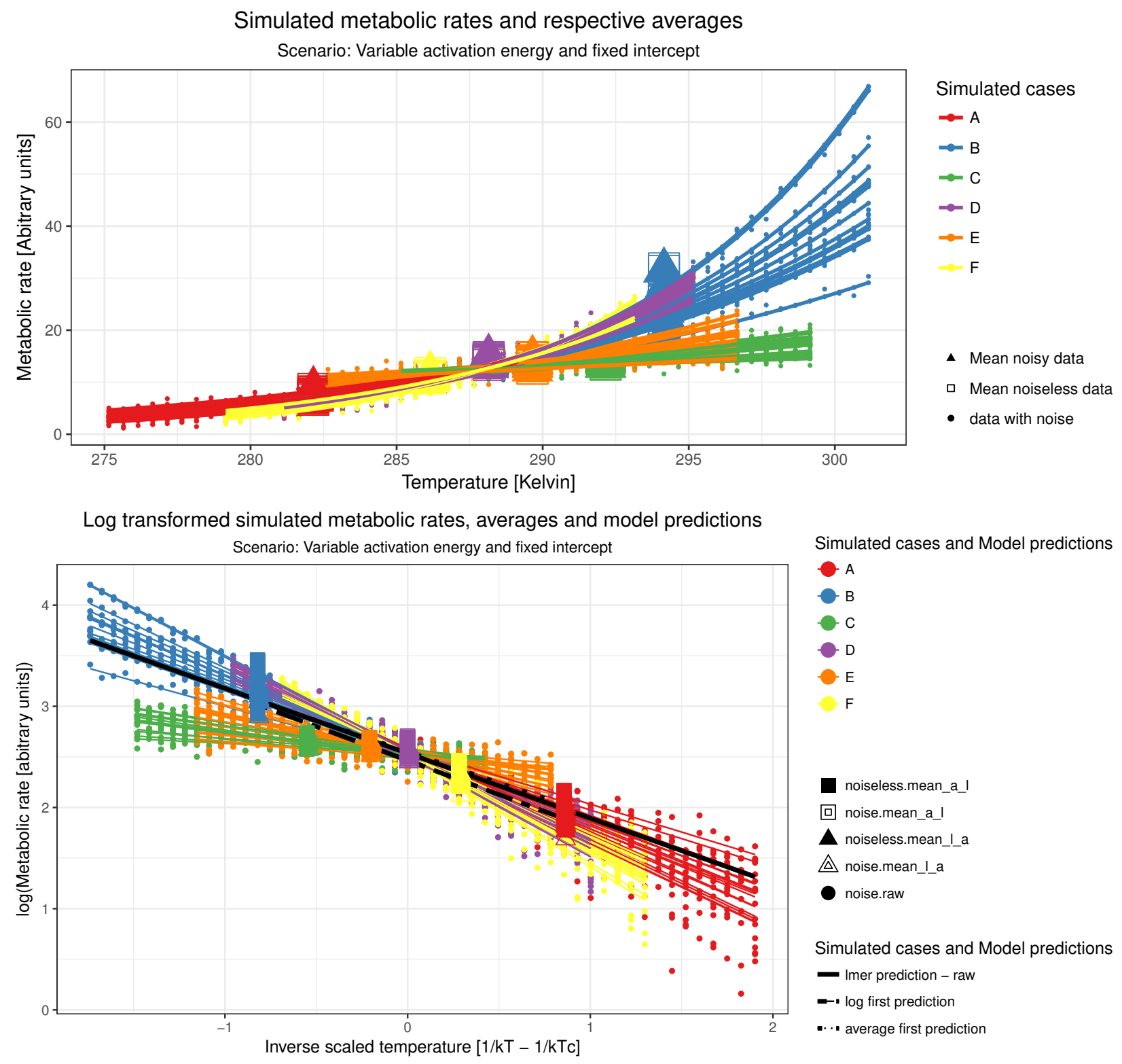


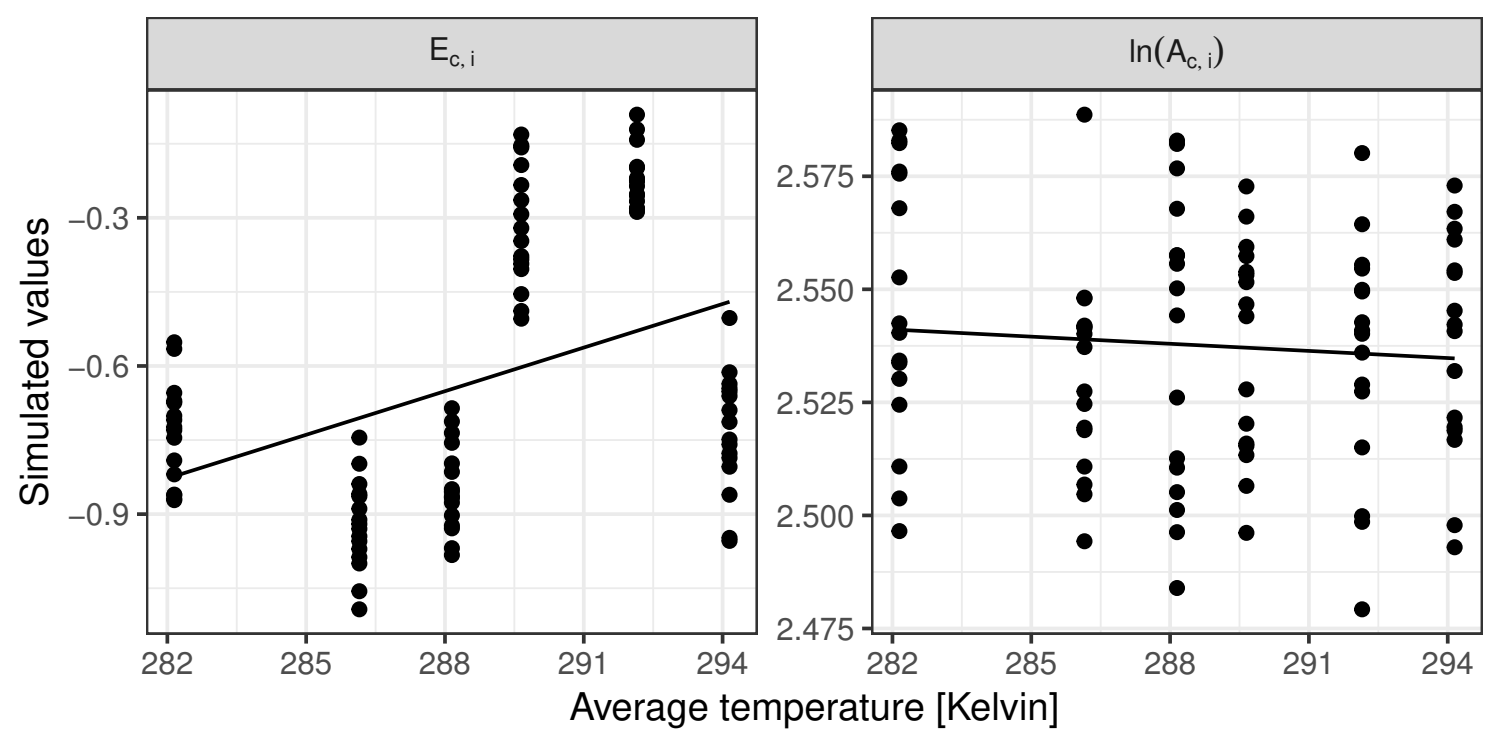

Figure 15: Scenario three: Regression of $\ln A_{c, i}$ and $E_{c, i}$ vs. $\overline{T_{c}}$ 


\subsection{Summary results scenario three:}

Estimated $\hat{E}$ by the $\log$-first approaches are mainly determined by $\overline{E_{c, i}}$, however, also $\beta_{E_{c, i}}$ is important (see table 27 and figure 18). This influential role of $\beta_{E_{c, i}}$ gets even greater for the average-first approach (see table 31 and figure 19). Contrary $\hat{E}$ estimated by the LMER-approach is almost exclusively determined by $\overline{E_{c, i}}$ (see table 35 and figure 20 ).

Estimated $\hat{A}$ from all three approaches are almost solely determined by $\overline{\ln A_{c, i}}$ (see tables $29,33,37$ and figures $18,19,20)$.

This results are consistent with a good representation of the distribution of $\overline{\ln A_{c, i}}$ by the distributions of $\hat{A}$ of all 3 methods (see figure 17). Still, the spread of the difference $\overline{\ln A_{c, i}}-\hat{A}$ is much higher for the average based approaches (see figure 16).

On the other hand the distributions of the $\hat{E}$ estimated by the average based approaches do not reproduce the distribution $\overline{E_{c, i}}$, while the one from the LMER approach does (see figure 17). In addition, the differences between $\overline{E_{c, i}}-\hat{E}$ have much smaller spread for the LMER approach (see figure 16).

\subsection{Scenario three: Comparison between $\overline{E_{c, i}}$ and $\overline{\ln A_{c, i}}$ and $\hat{E}$ and $\hat{A}$ from the 3 different estimation approaches}

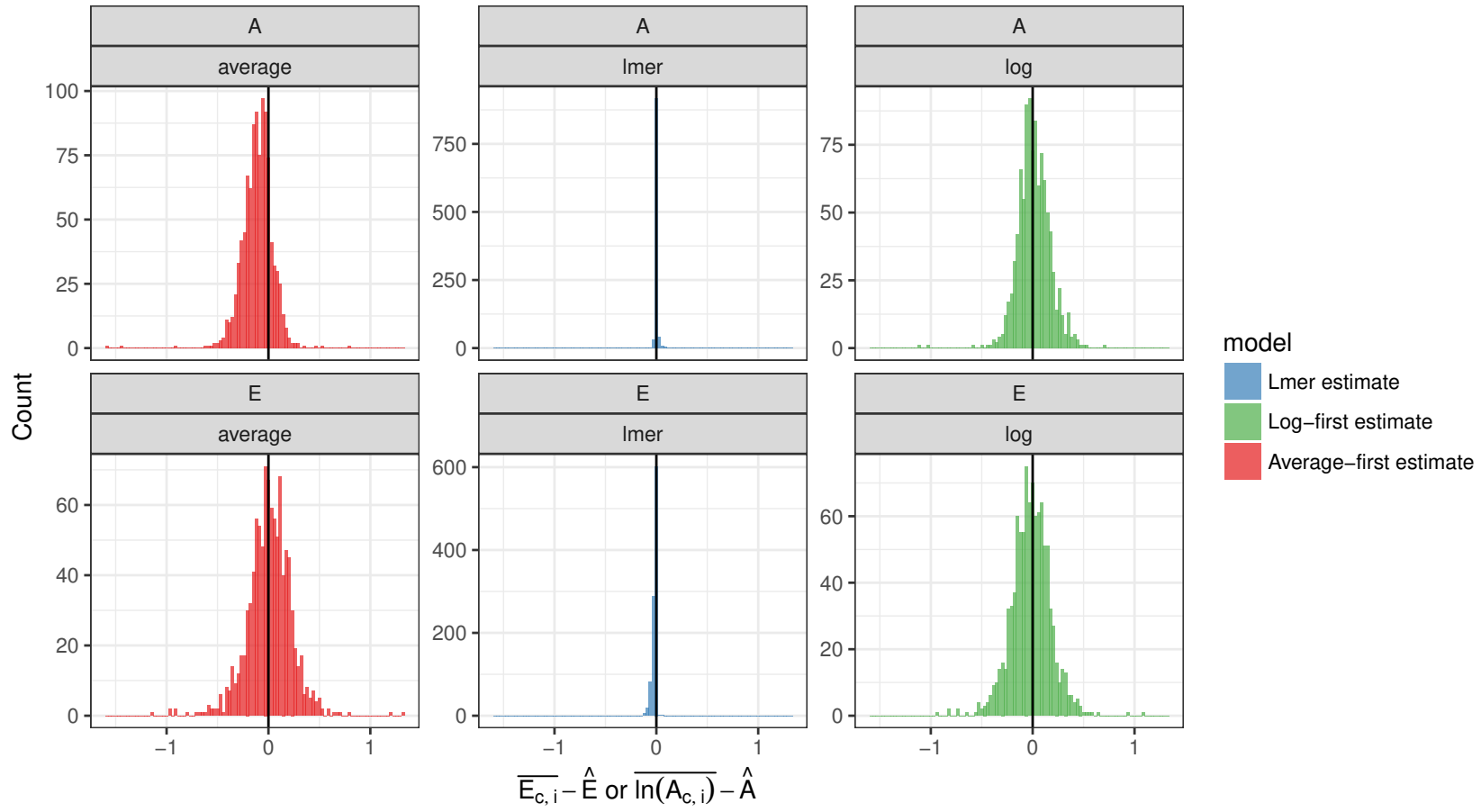

Figure 16: Scenario three: Comparison of $\overline{E_{c, i}}-\hat{E}$ and $\overline{\ln A_{c, i}}-\hat{A}$ for the 3 tested estimation approaches 


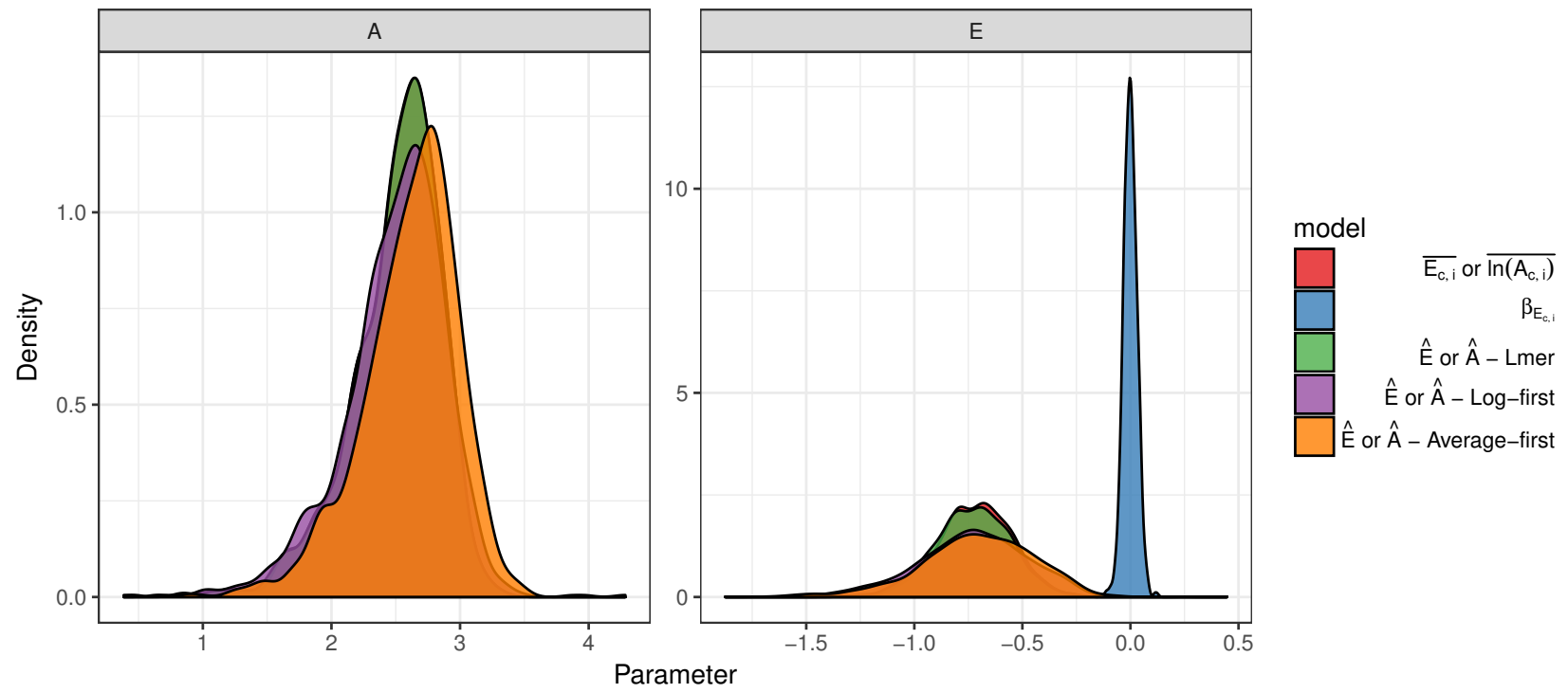

Figure 17: Scenario three: Comparison between simulated and estimated parameter 


\subsection{Scenario three: Detailed results on the influnece of chance correlation of}

$\ln A_{c, i} E_{c, i}$ with $\bar{T}_{c}$ on the estimates of $\hat{E}$ and $\hat{A}$

5.4.1 Log-first approach - Best model to explain $\hat{E}$ and $\hat{A}$ based on $\overline{E_{c, i}}, \overline{\ln A_{c, i}}, \beta_{E_{c, i}}$ and $\beta_{A_{c, i}}$
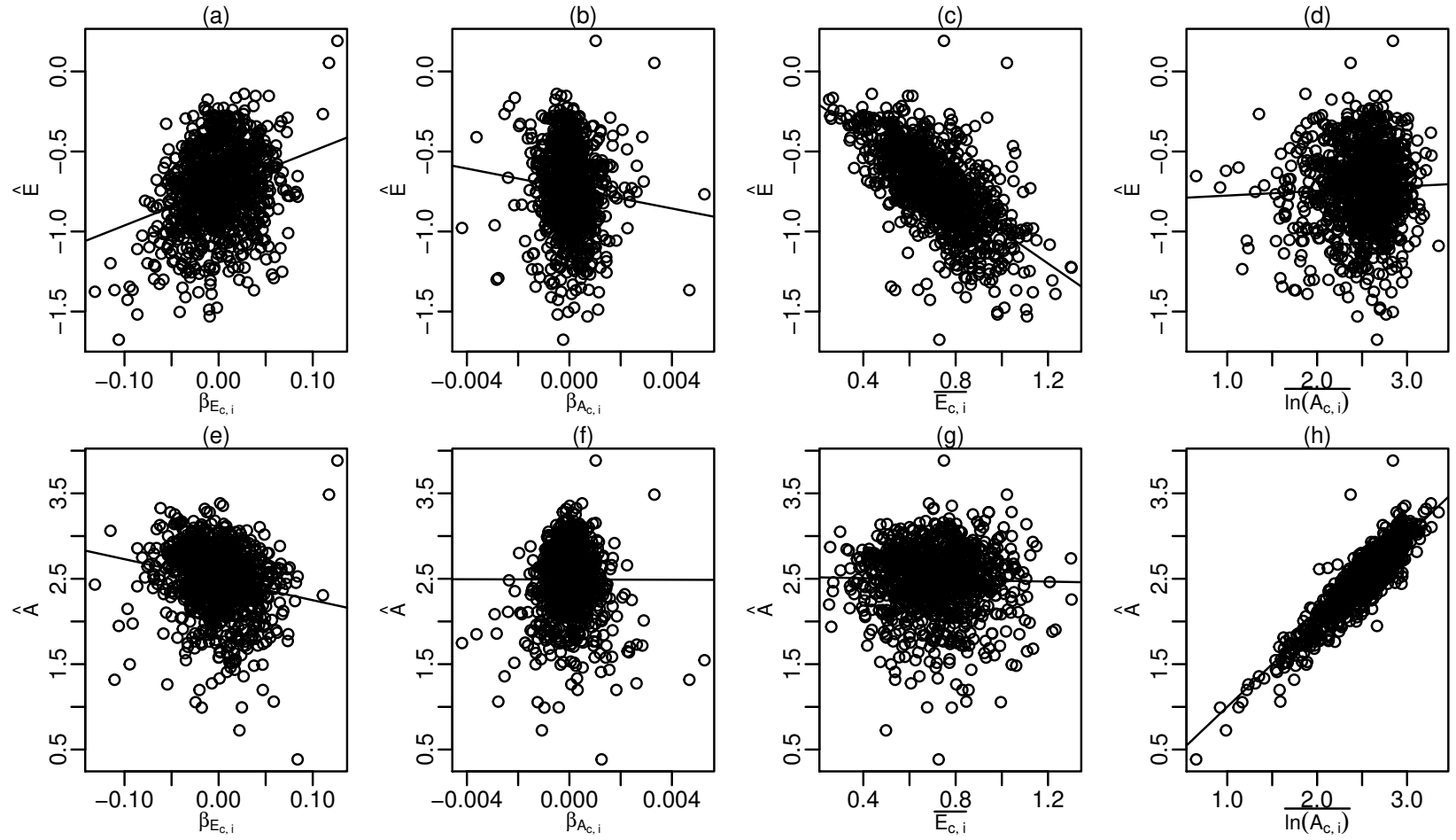

Figure 18: Scenario three - Log-first approach: Influence of $\beta_{E c, i}, \beta_{A c, i}, \overline{E_{c, i}}$, and $\overline{\ln A_{c, i}}$ on $\hat{E}$ and $\hat{A}$. Solid lines depict linear regression relations for all plot exept (c) and (h) were a bisection line is plotted since identity relations are expected.

Table 26: Scenario three - Log-first approach: Best model for $\hat{E}$

\begin{tabular}{lrrrr}
\hline & Estimate & Std. Error & t.value & $\operatorname{Pr}(>|t|)$ \\
\hline (Intercept) & -0.075 & 0.047 & -1.592 & 0.112 \\
\hline $\ln A_{c, i}$ & 0.025 & 0.016 & 1.540 & 0.124 \\
$\beta_{E_{c, i}}$ & 2.480 & 0.177 & 13.986 & 0.000 \\
\hline$E_{c, i}$ & -1.001 & 0.034 & -29.743 & 0.000 \\
\hline
\end{tabular}

Table 27: Scenario three - Log-first approach: Relative importance of best model parameters to explain $\hat{E}$

\begin{tabular}{lr}
\hline & Rel. Importance \\
\hline$\overline{\ln A_{c, i}}$ & 0.003 \\
$\beta_{E_{c, i}}$ & 0.173 \\
\hline$E_{c, i}$ & 0.824 \\
\hline
\end{tabular}


Table 28: Scenario three - Log-first approach: Best model for $\hat{A}$

\begin{tabular}{lrrrr}
\hline & Estimate & Std. Error & t.value & $\operatorname{Pr}(>|t|)$ \\
\hline (Intercept) & -0.100 & 0.031 & -3.166 & 0.002 \\
$\ln A_{c, i}$ & 1.033 & 0.012 & 83.173 & 0.000 \\
$\beta_{E_{c, i}}$ & -2.377 & 0.137 & -17.292 & 0.000 \\
\hline
\end{tabular}

Table 29: Scenario three - Log-first approach: Relative importance of best model parameters to explain $\hat{A}$

\begin{tabular}{lr}
\hline & Rel. Importance \\
\hline \hline $\ln A_{c, i}$ & 0.958 \\
$\beta_{E_{c, i}}$ & 0.042 \\
\hline
\end{tabular}


5.4.2 Average-first approach - Best model to explain $\hat{E}$ and $\hat{A}$ based on $\overline{E_{c, i}}, \overline{\ln A_{c, i}}, \beta_{E_{c, i}}$ and $\beta_{A_{c, i}}$
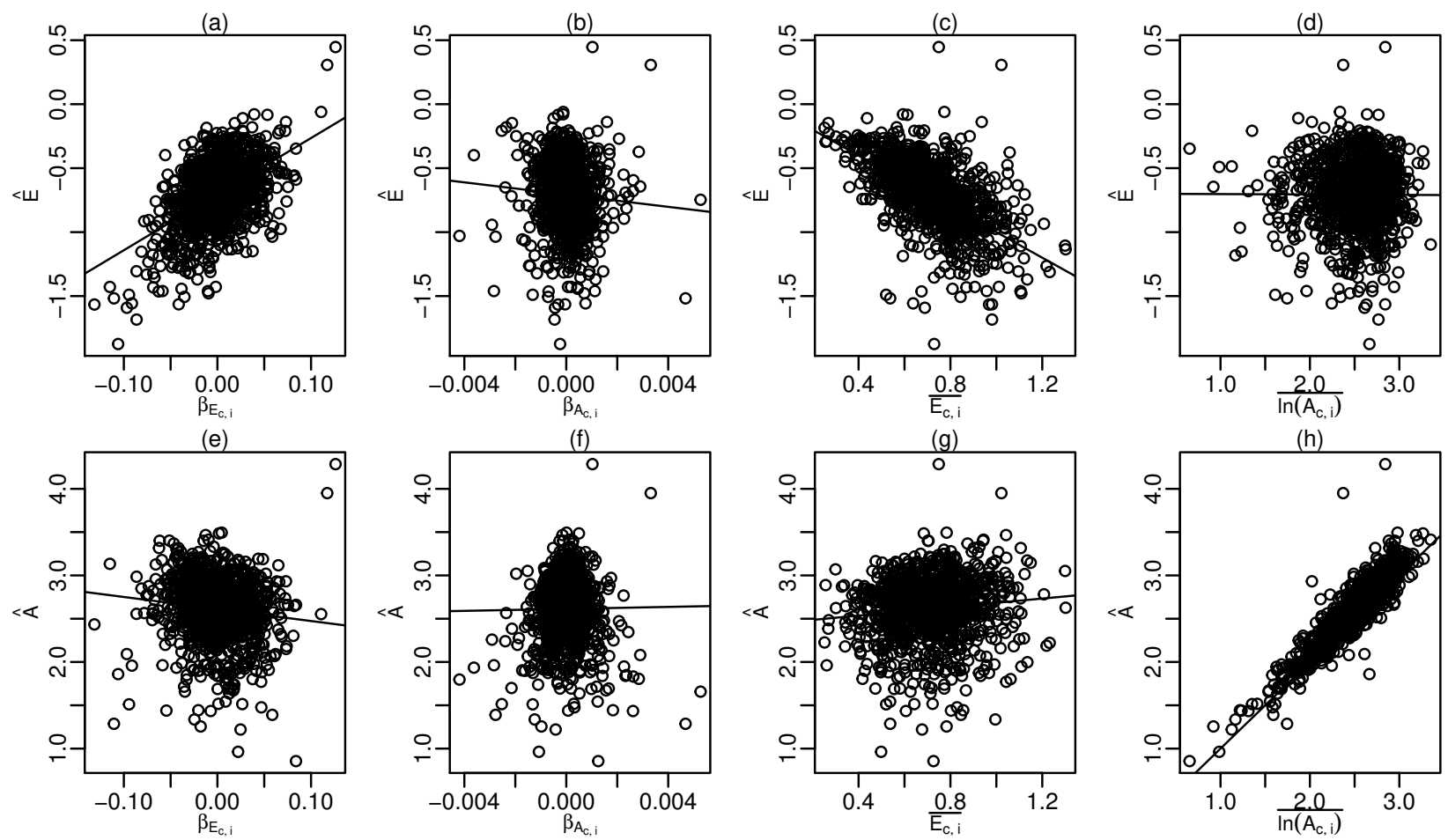

Figure 19: Scenario three - Average-first approach: Influence of $\beta_{E c, i}, \beta_{A c, i}, \overline{E_{c, i}}$, and $\overline{\ln A_{c, i}}$ on $\hat{E}$ and $\hat{A}$. Solid lines depict linear regression relations for all plot exept (c) and (h) were a bisection line is plotted since identity relations are expected.

Table 30: Scenario three - average-first approach: Best model for $\hat{E}$

\begin{tabular}{lrrrr}
\hline & Estimate & Std. Error & t.value & $\operatorname{Pr}(>|t|)$ \\
\hline (Intercept) & -0.031 & 0.023 & -1.319 & 0.187 \\
$\beta_{E_{c, i}}$ & 2.794 & 0.801 & 3.487 & 0.001 \\
\hline$E_{c, i}$ & -0.940 & 0.032 & -29.698 & 0.000 \\
$\beta_{E_{c, i}}: \overline{E_{c, i}}$ & 2.331 & 1.051 & 2.219 & 0.027 \\
\hline
\end{tabular}

Table 31: Scenario three - average-first approach: Relative importance of best model parameters to explain $\hat{E}$

\begin{tabular}{lr}
\hline & Rel. Importance \\
\hline$\beta_{E_{c, i}}$ & 0.452 \\
\hline$E_{c, i}$ & 0.544 \\
$\beta_{E_{c, i}}: \overline{E_{c, i}}$ & 0.003 \\
\hline
\end{tabular}


Table 32: Scenario three - average-first approach: Best model for $\hat{A}$

\begin{tabular}{lrrrr}
\hline & Estimate & Std. Error & t.value & $\operatorname{Pr}(>|t|)$ \\
\hline (Intercept) & -0.072 & 0.038 & -1.888 & 0.059 \\
$\ln A_{c, i}$ & 0.993 & 0.013 & 77.129 & 0.000 \\
$\beta_{E_{c, i}}$ & 0.212 & 0.977 & 0.217 & 0.828 \\
$\overline{E_{c, i}}$ & 0.273 & 0.027 & 10.113 & 0.000 \\
$\ln A_{c, i}: \beta_{E_{c, i}}$ & -0.649 & 0.388 & -1.675 & 0.094 \\
\hline
\end{tabular}

Table 33: Scenario three - average-first approach: Relative importance of best model parameters to explain $\hat{A}$

\begin{tabular}{lr}
\hline & Rel. Importance \\
\hline$\overline{\ln A_{c, i}}$ & 0.968 \\
$\beta_{E_{c, i}}$ & 0.016 \\
$\overline{E_{c, i}}$ & 0.016 \\
$\overline{\ln A_{c, i}}: \beta_{E_{c, i}}$ & 0.000 \\
\hline
\end{tabular}


5.4.3 LMER approach - Best model to explain $\hat{E}$ and $\hat{A}$ based on $\overline{E_{c, i}}, \overline{\ln A_{c, i}}, \beta_{E_{c, i}}$ and $\beta_{A_{c, i}}$
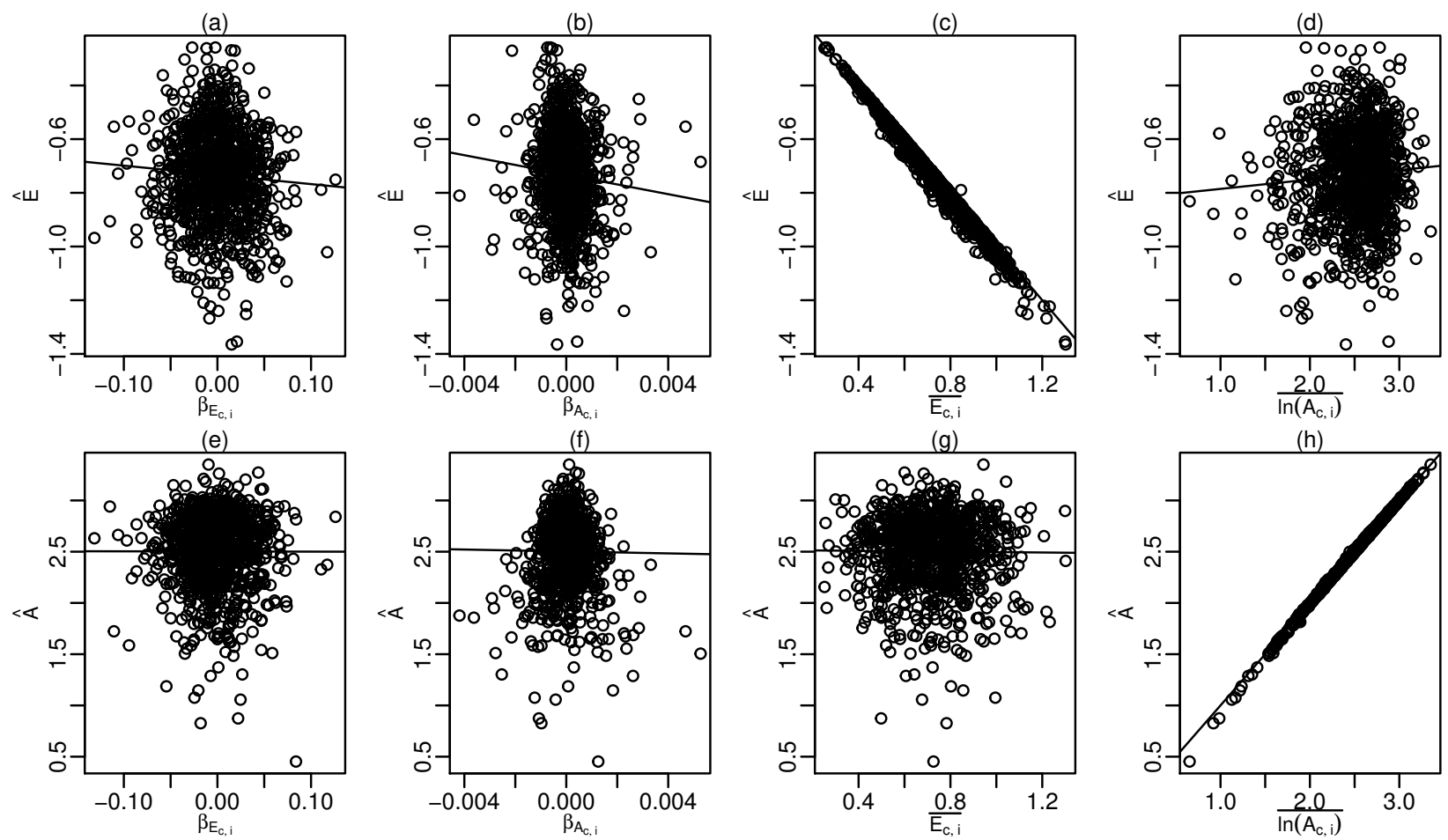

Figure 20: Scenario three - LMER approach: Influence of $\beta_{E c, i}, \beta_{A c, i}, \overline{E_{c, i}}$, and $\overline{\ln A_{c, i}}$ on $\hat{E}$ and $\hat{A}$. Solid lines depict linear regression relations for all plot exept (c) and (h) were a bisection line is plotted since identity relations are expected.

Table 34: Scenario three - lmer approach: Best model for $\hat{E}$

\begin{tabular}{lrrrr}
\hline & Estimate & Std. Error & t.value & $\operatorname{Pr}(>|t|)$ \\
\hline (Intercept) & -0.018 & 0.015 & -1.169 & 0.243 \\
$\overline{\ln A_{c, i}}$ & 0.011 & 0.006 & 1.872 & 0.062 \\
$\frac{\beta_{E_{c, i}}}{E_{c, i}}$ & -0.180 & 0.015 & -12.081 & 0.000 \\
$\overline{\ln A_{c, i}}: \overline{E_{c, i}}$ & -1.104 & 0.020 & -54.495 & 0.000 \\
\hline
\end{tabular}

Table 35: Scenario three - lmer approach: Relative importance of best model parameters to explain $\hat{E}$

\begin{tabular}{lr}
\hline & Rel. Importance \\
\hline$\overline{\ln A_{c, i}}$ & 0.004 \\
$\frac{\beta_{E_{c, i}}}{E_{c, i}}$ & 0.002 \\
$\overline{\ln A_{c, i}}: \overline{E_{c, i}}$ & 0.993 \\
\hline
\end{tabular}


Table 36: Scenario three - lmer approach: Best model for $\hat{A}$

\begin{tabular}{lrrrr}
\hline & Estimate & Std. Error & t.value & $\operatorname{Pr}(>|t|)$ \\
\hline (Intercept) & -0.014 & 0.011 & -1.283 & 0.2 \\
$\ln A_{c, i}$ & 1.004 & 0.004 & 237.478 & 0.0 \\
$\frac{\beta_{E_{c, i}}}{E_{c, i}}$ & -0.524 & 0.072 & -7.280 & 0.0 \\
$\overline{\ln A_{c, i}}: \beta_{E_{c, i}}$ & -0.054 & 0.014 & -3.801 & 0.0 \\
$\overline{\ln A_{c, i}: \overline{E_{c, i}}}$ & 0.214 & 0.029 & 7.484 & 0.0 \\
\hline
\end{tabular}

Table 37: Scenario three - lmer approach: Relative importance of best model parameters to explain $\hat{A}$

\begin{tabular}{lr}
\hline & Rel. Importance \\
\hline$\overline{\ln A_{c, i}}$ & 1 \\
$\frac{\beta_{E_{c, i}}}{\overline{E_{c, i}}}: \beta_{E_{c, i}}$ & 0 \\
$\overline{\ln A_{c, i}}: \overline{\ln A_{c, i}}: E_{c, i}$ & 0 \\
\hline
\end{tabular}




\section{Scenario four: All entities have variable absolute metabolic rates $A_{c}$ and activation energies $E_{c}$}

\subsection{Description and assumptions of scenario four}

In this scenario both $A_{c}$ and $E_{c}$ are assumed to vary randomly between the entities. Thus, both ln $A_{c, i}$ and $E_{c, i}$ can by chance exhibit a correlation with $\bar{T}_{c}$ (see figure 21 ). This scenario is likely the most realistic one. Since random variation is assumed for both parameters it is justified that in a regression approach we are interested in an average effect for both parameters. The assumptions in this scenario are identical to the ones made to analyse the mesocosm data.
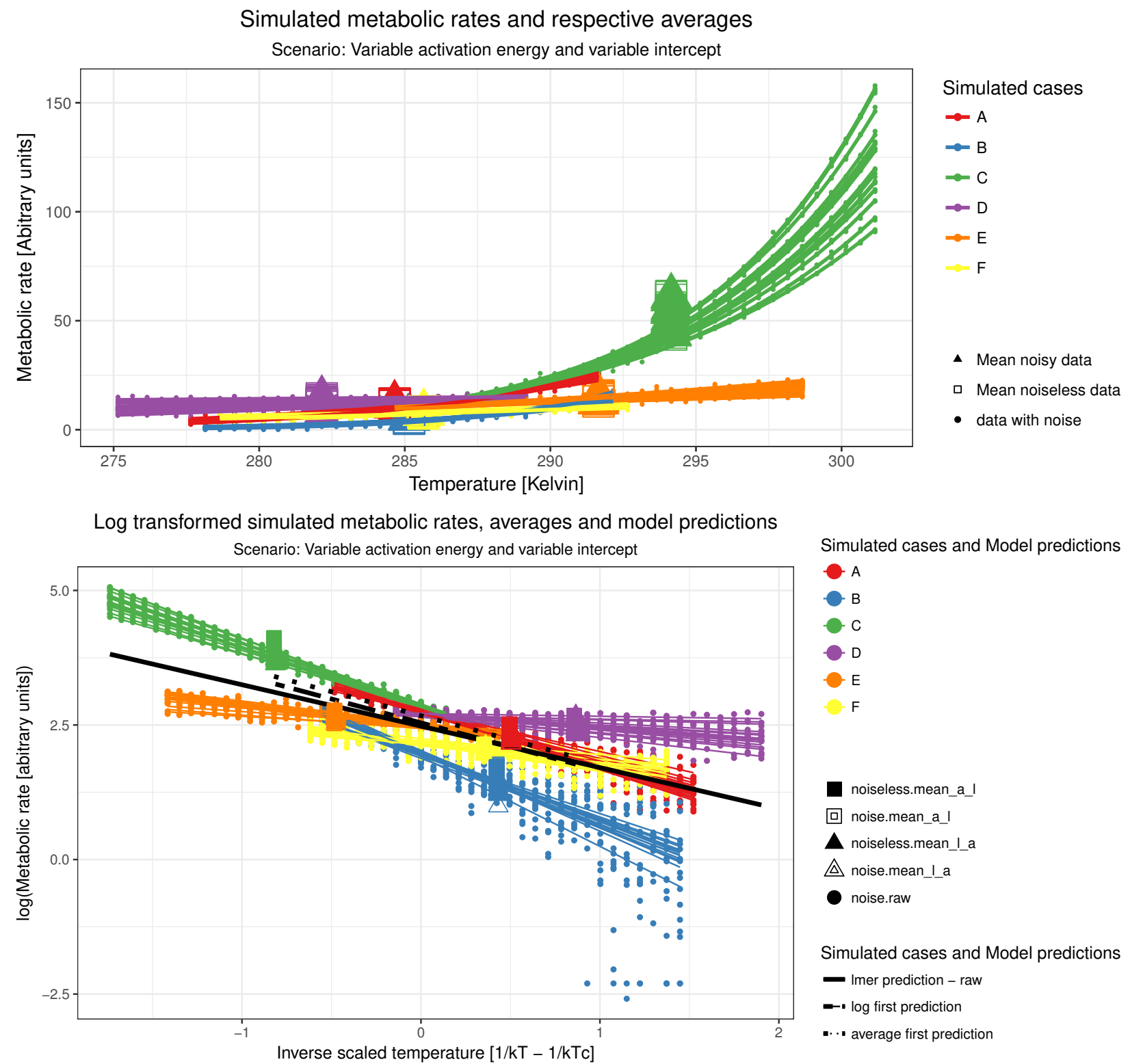

Simulated cases and Model predictions

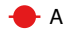

B

C

- D

- $E$

$\mathrm{F}$

noiseless.mean_a_I

回 noise.mean_a_।

A noiseless.mean_la

$\triangle$ noise.mean_l_a

noise.raw

Simulated cases and Model predictions

- Imer prediction - raw

- $\log$ first prediction

.... average first prediction 


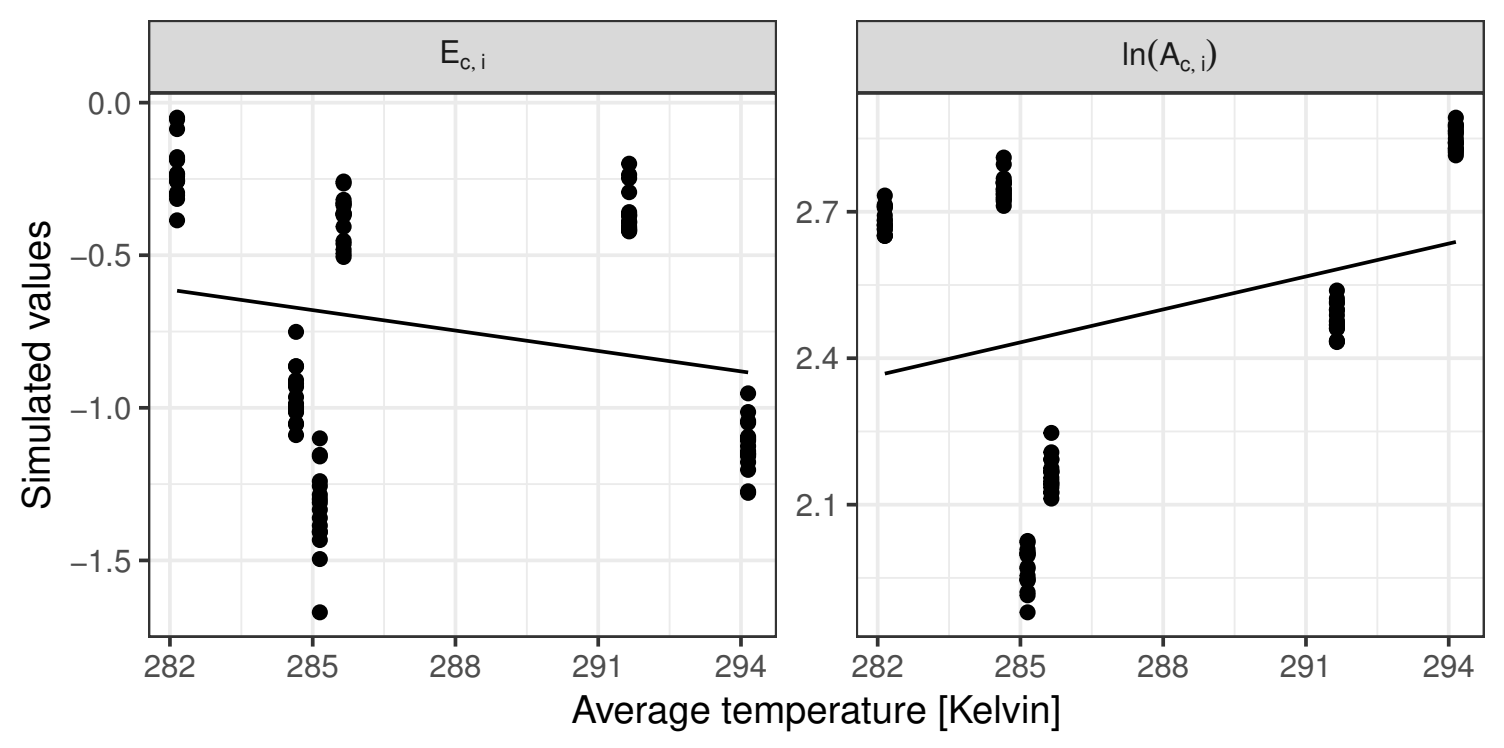

Figure 21: Scenario four: Regression of $\ln A_{c, i}$ and $E_{c, i}$ vs. $\overline{T_{c}}$ 


\subsection{Summary results scenario four:}

For both average based approaches $\hat{E}$ are heavily influenced by $\beta_{A_{c, i}}$, which seems to be even more important than the value of $\overline{E_{c, i}}$ itself (see tables 39, 43 and figures 24,25 ). In addition, $\hat{E}$ by the average first approach is also influenced by $\beta_{E_{c, i}}$ (see table 43 and figure 25). Contrary $\hat{E}$ estimated by the LMER approach is determined by $\overline{E_{c, i}}$ only (see table 47 and figure 26 ).

The estimates of $\hat{A}$ based the two average based methods are mainly driven by $\overline{\ln A_{c, i}}$, but also by $\beta_{A_{c, i}}$ and for the log-first approach also by $\beta_{E_{c, i}}$ (see tables 41,45 and figures 24,25 ). $\hat{A}$ estimated by LMER is almost exclusively determined by $\overline{\ln A_{c, i}}$ (see table 49 and figure 26). This results coincide with a by far smallest spread of $\overline{\ln A_{c, i}}-\hat{A}$ and $\overline{E_{c, i}}-\hat{E}$ for the LMER approach (see figure 22). Also, only estimates of the LMER approach capture the density distributions of $\overline{\ln A_{c, i}}$ and $\overline{E_{c, i}}$ (see figure 23). It is important to note that the average based approaches do not convey any "new" or "extra" information, which LMER does not capture but only seem to estimate $\overline{E_{c, i}}$ and $\overline{\ln A_{c, i}}$ very badly, most likely due to the heavy influence of $\beta_{A_{c, i}}$ and $\beta_{E_{c, i}}$.

\subsection{Scenario four: Comparison between $\overline{E_{c, i}}$ and $\overline{\ln A_{c, i}}$ and $\hat{E}$ and $\hat{A}$ from the 3 different estimation approaches}
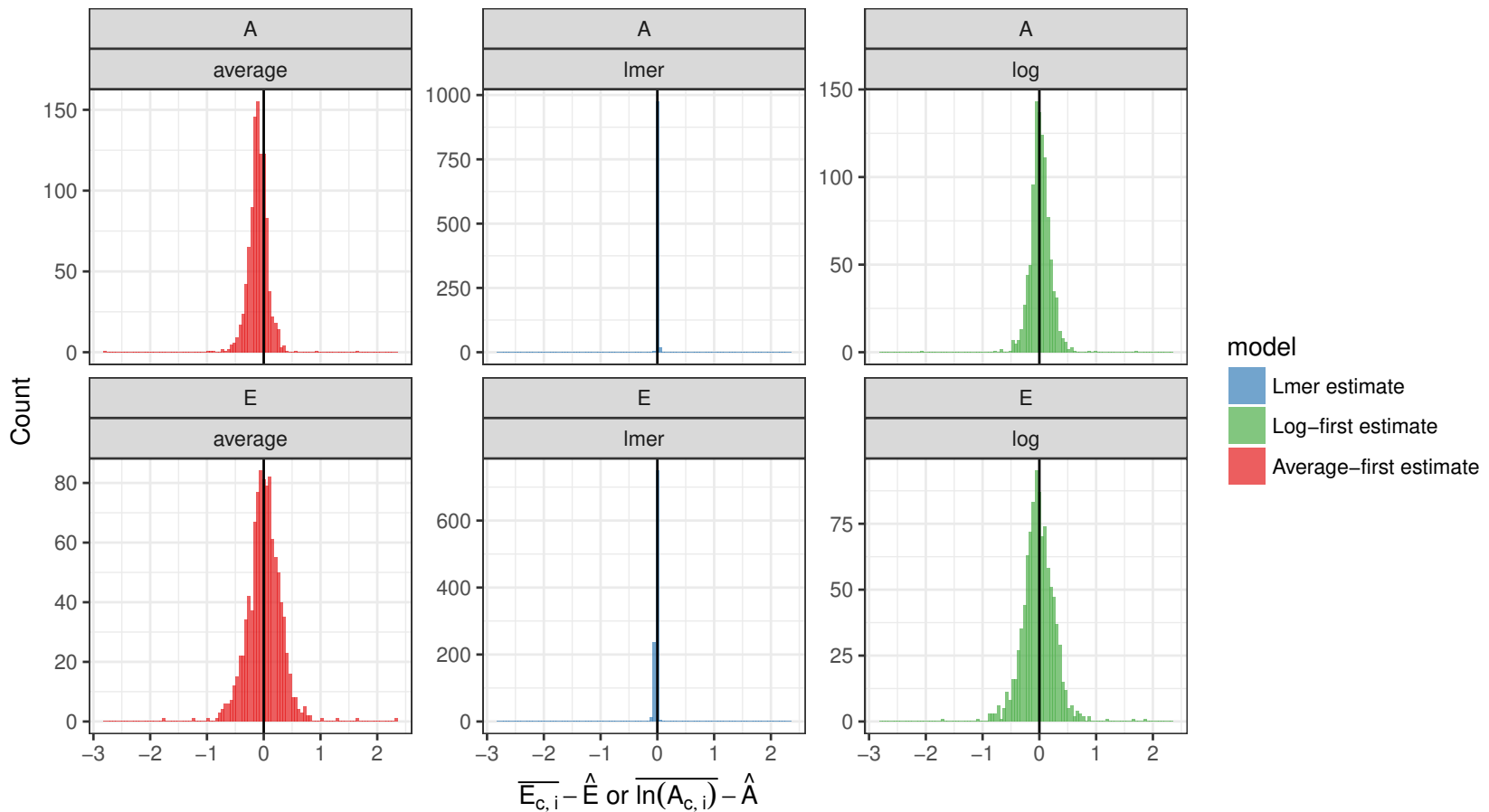

Figure 22: Scenario three: Comparison of $\overline{E_{c, i}}-\hat{E}$ and $\overline{\ln A_{c, i}}-\hat{A}$ for the 3 tested estimation approaches 

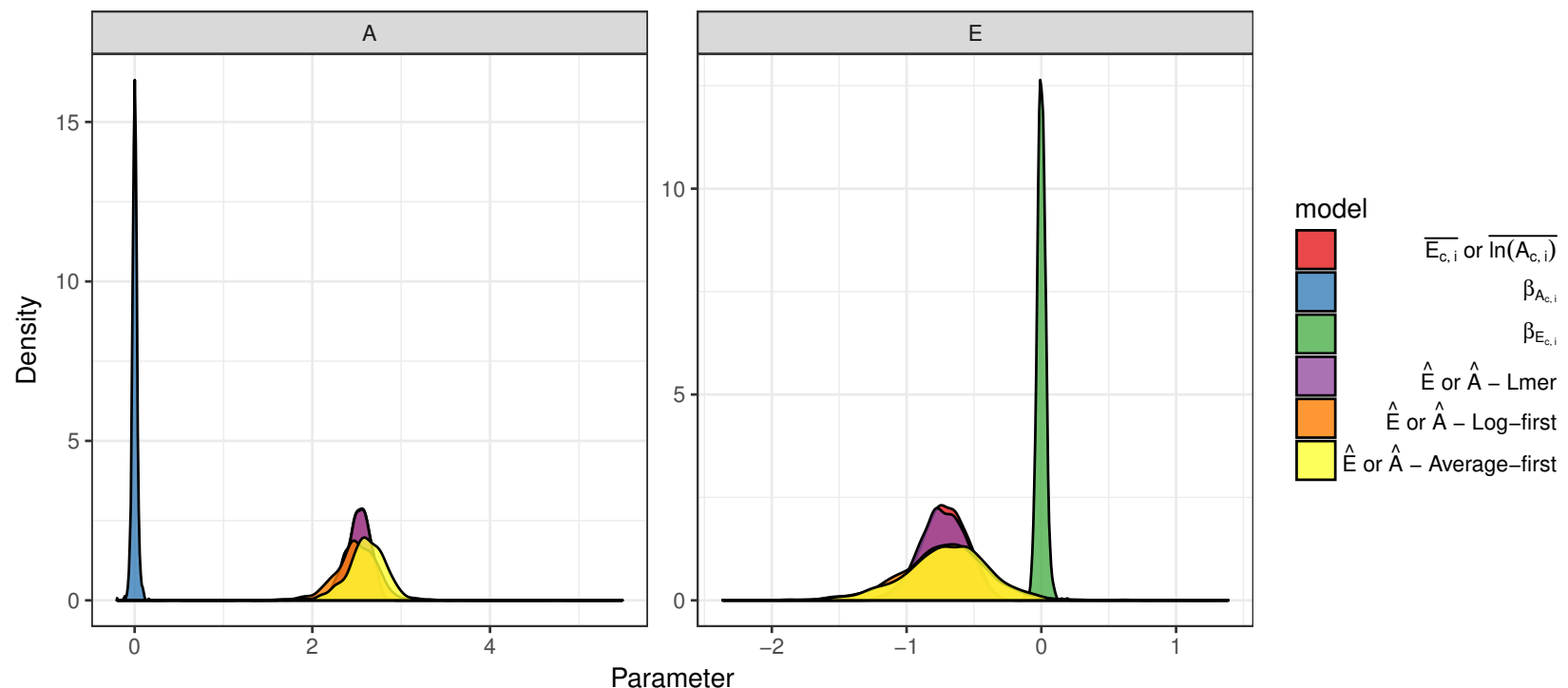

Figure 23: Scenario four: Comparison between simulated and estimated parameter 


\subsection{Scenario four: Detailed results on the influnece of chance correlation of $\ln A_{c, i} E_{c, i}$ with $\bar{T}_{c}$ on the estimates of $\hat{E}$ and $\hat{A}$}

6.4.1 Log-first approach - Best model to explain $\hat{E}$ and $\hat{A}$ based on $\overline{E_{c, i}}, \overline{\ln A_{c, i}}, \beta_{E_{c, i}}$ and $\beta_{A_{c, i}}$
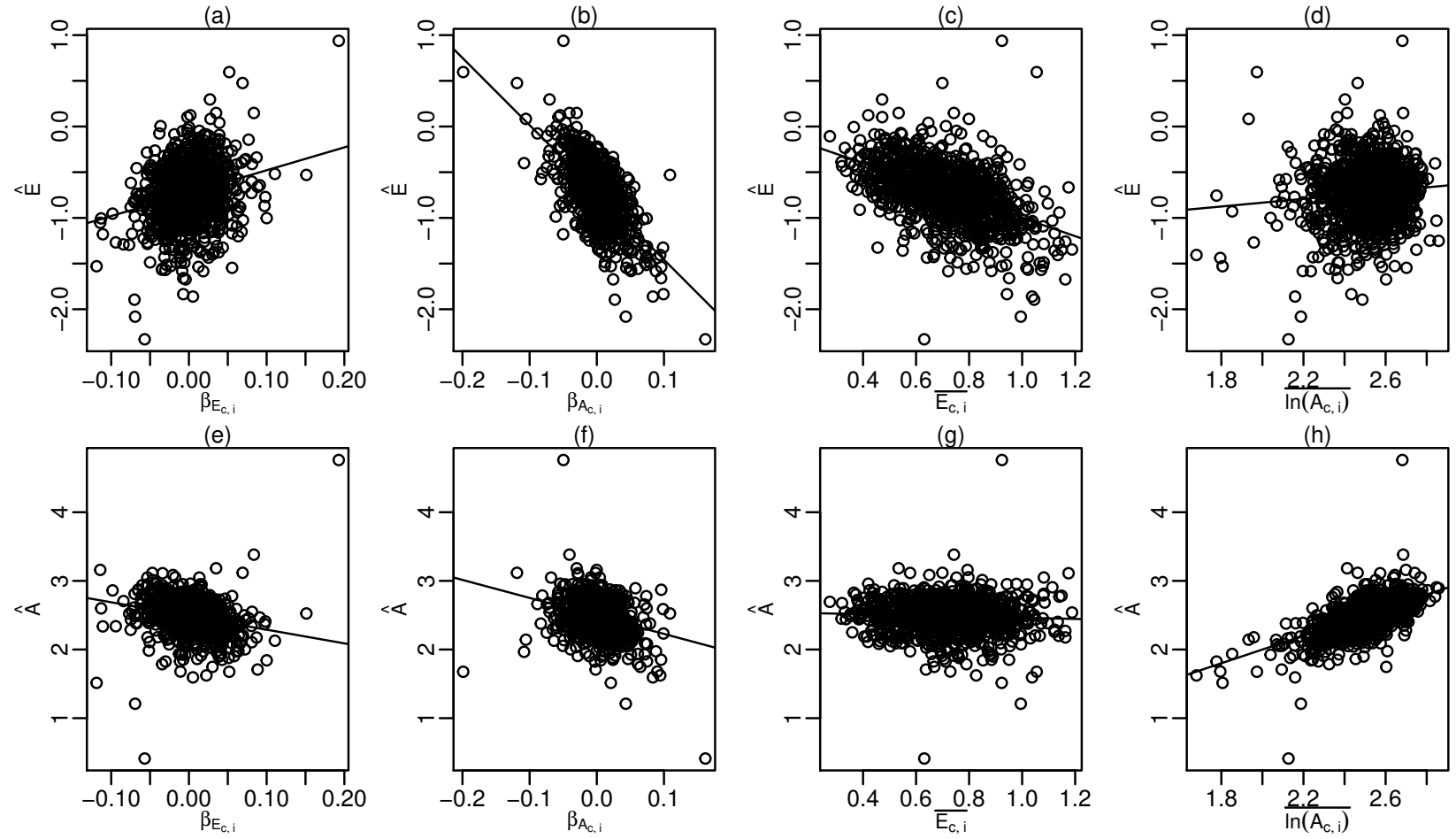

Figure 24: Scenario four - Log-first approach: Influence of $\beta_{E c, i}, \beta_{A c, i}, \overline{E_{c, i}}$, and $\overline{\ln A_{c, i}}$ on $\hat{E}$ and $\hat{A}$. Solid lines depict linear regression relations for all plot exept (c) and (h) were a bisection line is plotted since identity relations are expected.

Table 38: Scenario four - Log-first approach: Best model for $\hat{E}$

\begin{tabular}{|c|c|c|c|c|}
\hline & Estimate & Std. Error & t.value & $\operatorname{Pr}(>|t|)$ \\
\hline (Intercept) & 0.841 & 0.435 & 1.933 & 0.054 \\
\hline$\overline{\ln A_{c, i}}$ & .333 & 0.173 & -1.930 & 0.054 \\
\hline$\beta_{A_{c, i}}$ & -7.336 & 0.191 & -38.460 & 0.000 \\
\hline$\beta_{E_{c, i}}$ & -5.407 & 2.629 & -2.057 & 0.040 \\
\hline$\frac{E_{c, i}}{E_{c, i}}$ & -2.320 & 0.582 & -3.983 & 0.000 \\
\hline $\ln A_{c, i}: \beta_{E_{c, i}}$ & 1.784 & 0.990 & 1.803 & 0.072 \\
\hline $\ln A_{c, i}: \overline{E_{c, i}}$ & 0.517 & 0.231 & 2.237 & 0.026 \\
\hline$\beta_{E_{c, i}}: \overline{E_{c, i}}$ & 4.247 & 1.118 & 3.799 & 0.000 \\
\hline
\end{tabular}

Table 39: Scenario four - Log-first approach: Relative importance of best model parameters to explain $\hat{E}$

\begin{tabular}{lr}
\hline & Rel. Importance \\
\hline$\overline{\ln A_{c, i}}$ & 0.008 \\
$\beta_{A_{c, i}}$ & 0.567
\end{tabular}




\begin{tabular}{lr}
\hline & Rel. Importance \\
\hline$\beta_{E_{c, i}}$ & 0.079 \\
$\overline{E_{c, i}}$ & 0.339 \\
$\overline{\ln A_{c, i}}: \beta_{E_{c, i}}$ & 0.001 \\
$\overline{\ln A_{c, i}}: \overline{E_{c, i}}$ & 0.001 \\
$\beta_{E_{c, i}}: \bar{E}_{c, i}$ & 0.006 \\
\hline
\end{tabular}

Table 40: Scenario four - Log-first approach: Best model for $\hat{A}$

\begin{tabular}{lrrrr}
\hline & Estimate & Std. Error & t.value & $\operatorname{Pr}(>|t|)$ \\
\hline (Intercept) & 1.159 & 0.427 & 2.713 & 0.007 \\
$\ln A_{c, i}$ & 0.548 & 0.170 & 3.230 & 0.001 \\
$\beta_{A_{c, i}}$ & -3.863 & 0.847 & -4.560 & 0.000 \\
$\frac{\beta_{E_{c, i}}}{E_{c, i}}$ & -5.273 & 0.840 & -6.279 & 0.000 \\
$\overline{\ln A_{c, i}}: \overline{E_{c, i}}$ & -1.920 & 0.572 & -3.357 & 0.001 \\
$\beta_{A_{c, i}}: \overline{E_{c, i}}$ & 0.743 & 0.227 & 3.271 & 0.001 \\
$\beta_{E_{c, i}}: \overline{E_{c, i}}$ & 2.083 & 1.109 & 1.878 & 0.061 \\
\hline
\end{tabular}

Table 41: Scenario four - Log-first approach: Relative importance of best model parameters to explain $\hat{A}$

\begin{tabular}{lr}
\hline & Rel. Importance \\
\hline$\overline{\ln A_{c, i}}$ & 0.722 \\
$\beta_{A_{c, i}}$ & 0.132 \\
$\beta_{E_{c, i}}$ & 0.122 \\
$\overline{E_{c, i}}$ & 0.004 \\
$\overline{\ln A_{c, i}}: \overline{E_{c, i}}$ & 0.007 \\
$\beta_{A_{c, i}}: \overline{E_{c, i}}$ & 0.003 \\
$\beta_{E_{c, i}}: \overline{E_{c, i}}$ & 0.010 \\
\hline
\end{tabular}


6.4.2 Average-first approach - Best model to explain $\hat{E}$ and $\hat{A}$ based on $\overline{E_{c, i}}, \overline{\ln A_{c, i}}, \beta_{E_{c, i}}$ and $\beta_{A_{c, i}}$
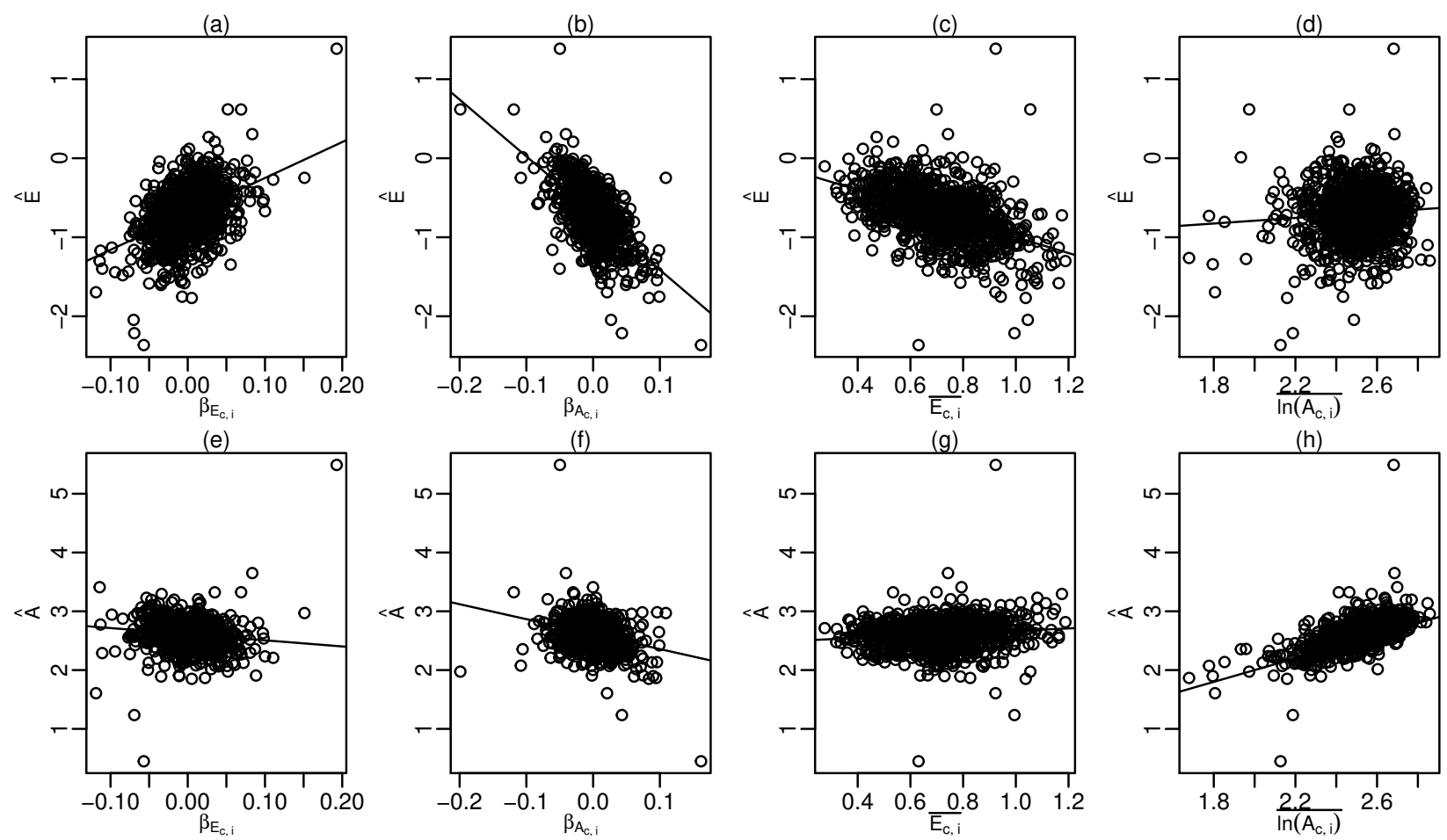

Figure 25: Scenario four - Average-first approach: Influence of $\beta_{E c, i}, \beta_{A c, i}, \overline{E_{c, i}}$, and $\overline{\ln A_{c, i}}$ on $\hat{E}$ and $\hat{A}$. Solid lines depict linear regression relations for all plot exept (c) and (h) were a bisection line is plotted since identity relations are expected.

Table 42: Scenario four - average-first approach: Best model for $\hat{E}$

\begin{tabular}{lrrrr}
\hline & Estimate & Std. Error & t.value & $\operatorname{Pr}(>|t|)$ \\
\hline (Intercept) & 0.904 & 0.408 & 2.216 & 0.027 \\
$\frac{\ln A_{c, i}}{\beta_{A_{c, i}}}$ & -0.364 & 0.162 & -2.248 & 0.025 \\
$\frac{\beta_{E_{c, i}}}{E_{c, i}}$ & -7.033 & 0.179 & -39.317 & 0.000 \\
$\frac{\ln A_{c, i}}{\ln A_{c, i}: \beta_{E_{c, i}}: E_{c, i}}$ & -4.712 & 2.466 & -1.911 & 0.056 \\
$\beta_{E_{c, i}}: \overline{E_{c, i}}$ & -2.247 & 0.546 & -4.113 & 0.000 \\
\hline
\end{tabular}

Table 43: Scenario four - average-first approach: Relative importance of best model parameters to explain $\hat{E}$

\begin{tabular}{lr}
\hline & Rel. Importance \\
\hline$\overline{\ln A_{c, i}}$ & 0.004 \\
$\beta_{A_{c, i}}$ & 0.474 \\
$\beta_{E_{c, i}}$ & 0.240 \\
\hline$E_{c, i}$ & 0.271
\end{tabular}




\begin{tabular}{lr}
\hline & Rel. Importance \\
\hline$\overline{\ln A_{c, i}}: \beta_{E_{c, i}}$ & 0.001 \\
$\overline{\ln A_{c, i}: \overline{E_{c, i}}}$ & 0.001 \\
$\beta_{E_{c, i}}: \overline{E_{c, i}}$ & 0.009 \\
\hline
\end{tabular}

Table 44: Scenario four - average-first approach: Best model for $\hat{A}$

\begin{tabular}{lrrrr}
\hline & Estimate & Std. Error & t.value & $\operatorname{Pr}(>|t|)$ \\
\hline (Intercept) & 1.158 & 0.449 & 2.581 & 0.010 \\
$\overline{\ln A_{c, i}}$ & 0.516 & 0.178 & 2.896 & 0.004 \\
$\beta_{A_{c, i}}$ & -3.842 & 0.890 & -4.318 & 0.000 \\
$\frac{\beta_{E_{c, i}}}{\overline{E_{c, i}}}$ & -5.259 & 0.882 & -5.962 & 0.000 \\
$\overline{\ln A_{c, i}}: \overline{E_{c, i}}$ & -1.596 & 0.601 & -2.656 & 0.008 \\
$\beta_{A_{c, i}}: \overline{E_{c, i}}$ & 0.727 & 0.239 & 3.048 & 0.002 \\
$\beta_{E_{c, i}}: \overline{E_{c, i}}$ & 2.262 & 1.165 & 1.942 & 0.052 \\
\hline
\end{tabular}

Table 45: Scenario four - average-first approach: Relative importance of best model parameters to explain $\hat{A}$

\begin{tabular}{lr}
\hline & Rel. Importance \\
\hline$\overline{\ln A_{c, i}}$ & 0.754 \\
$\beta_{A_{c, i}}$ & 0.136 \\
$\beta_{E_{c, i}}$ & 0.043 \\
$\overline{E_{c, i}}$ & 0.037 \\
$\overline{\ln A_{c, i}}: \overline{E_{c, i}}$ & 0.008 \\
$\beta_{A_{c, i}}: \overline{E_{c, i}}$ & 0.004 \\
$\beta_{E_{c, i}}: \overline{E_{c, i}}$ & 0.019 \\
\hline
\end{tabular}


6.4.3 LMER approach - Best model to explain $\hat{E}$ and $\hat{A}$ based on $\overline{E_{c, i}}, \overline{\ln A_{c, i}}, \beta_{E_{c, i}}$ and $\beta_{A_{c, i}}$
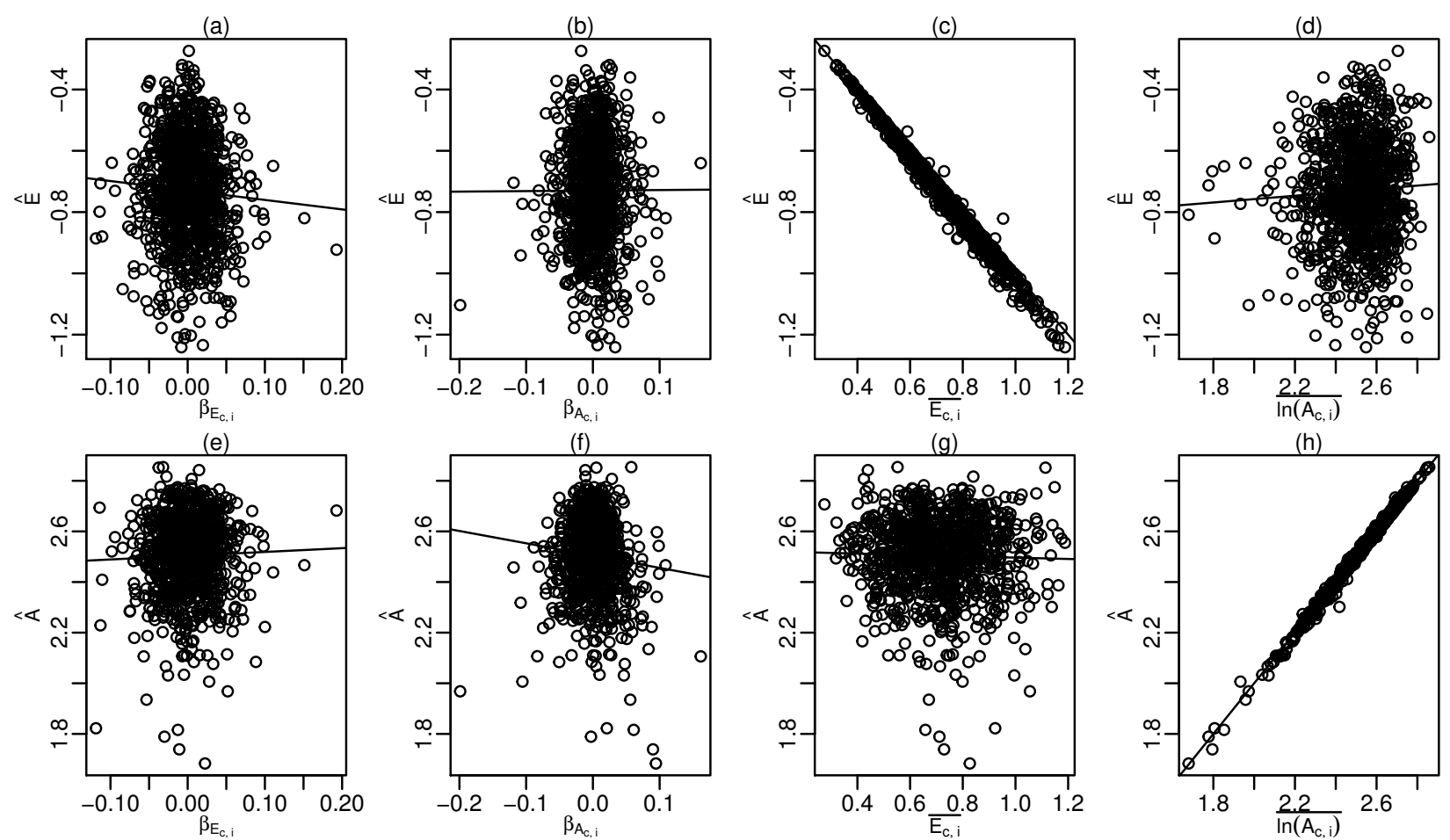

Figure 26: Scenario four - LMER approach: Influence of $\beta_{E c, i}, \beta_{A c, i}, \overline{E_{c, i}}$, and $\overline{\ln A_{c, i}}$ on $\hat{E}$ and $\hat{A}$. Solid lines depict linear regression relations for all plot exept (c) and (h) were a bisection line is plotted since identity relations are expected.

Table 46: Scenario four - lmer approach: Best model for $\hat{E}$

\begin{tabular}{lrrrr}
\hline & Estimate & Std. Error & t.value & $\operatorname{Pr}(>|t|)$ \\
\hline (Intercept) & -0.038 & 0.010 & -3.933 & 0.000 \\
$\frac{\ln A_{c, i}}{\beta_{A_{c, i}}}$ & 0.020 & 0.004 & 5.360 & 0.000 \\
$\beta_{E_{c, i}}$ & 1.211 & 0.261 & 4.641 & 0.000 \\
$\overline{E_{c, i}}$ & -0.761 & 0.249 & -3.055 & 0.002 \\
$\overline{\ln A_{c, i}}: \beta_{A_{c, i}}$ & -1.041 & 0.003 & -311.565 & 0.000 \\
$\overline{\ln A_{c, i}}: \beta_{E_{c, i}}$ & -0.464 & 0.093 & -5.004 & 0.000 \\
$\beta_{A_{c, i}}: \bar{E}_{c, i}$ & -0.224 & 0.100 & 2.249 & 0.025 \\
\hline
\end{tabular}

Table 47: Scenario four - lmer approach: Relative importance of best model parameters to explain $\hat{E}$

\begin{tabular}{lr}
\hline & Rel. Importance \\
\hline$\overline{\ln A_{c, i}}$ & 0.001 \\
$\beta_{A_{c, i}}$ & 0.000 \\
$\overline{\beta_{E_{c, i}}}$ & 0.002 \\
$\overline{E_{c, i}}$ & 0.996 \\
$\ln A_{c, i}: \beta_{A_{c, i}}$ & 0.000
\end{tabular}




\begin{tabular}{lr}
\hline & Rel. Importance \\
\hline$\overline{\ln A_{c, i}}: \beta_{E_{c, i}}$ & 0.000 \\
$\beta_{A_{c, i}}: E_{c, i}$ & 0.000 \\
\hline
\end{tabular}

Table 48: Scenario four - lmer approach: Best model for $\hat{A}$

\begin{tabular}{|c|c|c|c|c|}
\hline & Estimate & Std. Error & t.value & $\operatorname{Pr}(>|t|)$ \\
\hline (Intercept) & -0.042 & 0.005 & -8.072 & 0.000 \\
\hline$\overline{\ln A_{c, i}}$ & 1.015 & 0.002 & 509.080 & 0.000 \\
\hline$\beta_{A_{c, i}}$ & -0.735 & 0.118 & -6.231 & 0.000 \\
\hline$\beta_{E_{c, i}}$ & -0.355 & 0.136 & -2.599 & 0.009 \\
\hline$E_{c, i}$ & 0.005 & 0.002 & 2.510 & 0.012 \\
\hline$\overline{\ln A_{c, i}}: \beta_{A_{c, i}}$ & 0.288 & 0.049 & 5.880 & 0.000 \\
\hline$\overline{\ln A_{c, i}}: \beta_{E_{c, i}}$ & 0.154 & 0.055 & 2.815 & 0.005 \\
\hline
\end{tabular}

Table 49: Scenario four - lmer approach: Relative importance of best model parameters to explain $\hat{A}$

\begin{tabular}{lr}
\hline & Rel. Importance \\
\hline$\overline{\ln A_{c, i}}$ & 0.995 \\
$\beta_{A_{c, i}}$ & 0.004 \\
$\frac{\beta_{E_{c, i}}}{E_{c, i}}$ & 0.000 \\
$\overline{\ln A_{c, i}}: \beta_{A_{c, i}}$ & 0.000 \\
$\overline{\ln A_{c, i}}: \beta_{E_{c, i}}$ & 0.000 \\
\hline
\end{tabular}




\section{Scenario five: All entities are allowed to have variable $E_{c}$ and $A_{c}$. Where $E_{c}$ are negatively correlated with $\bar{T}_{c}$}

\subsection{Description and assumptions of scenario five}

In this scenario entities have random variation in $A_{c}$, while $E_{c}$, even so first randomly drown, are sorted such that they decrease with increasing $\bar{T}_{c}$ (see figure 27). Since random variation is only assumed for parameter $A$, but $E$ changes systematical, it seems questionable if a average response of $\hat{E}$ over the temperature gradient is of any core interest. Rather, if such a systematic decrease of $E_{c}$ with $\bar{T}_{c}$ is hypothesized entities of the higher temperature ranges stand for future systems, while the ones from colder temperature ranges stand for contemporary ones. Thus, the focus of interest would be to qualify and quantify the systematic change, i.e. $\hat{E}_{c} \sim \bar{T}_{c}$. Still, the question is if either LMER or average based approaches are able to capture meaning full information about such a systematic change and if the average based approaches can capture any of the dynamics which the LMER approach could not?

\section{Simulated metabolic rates and respective averages}

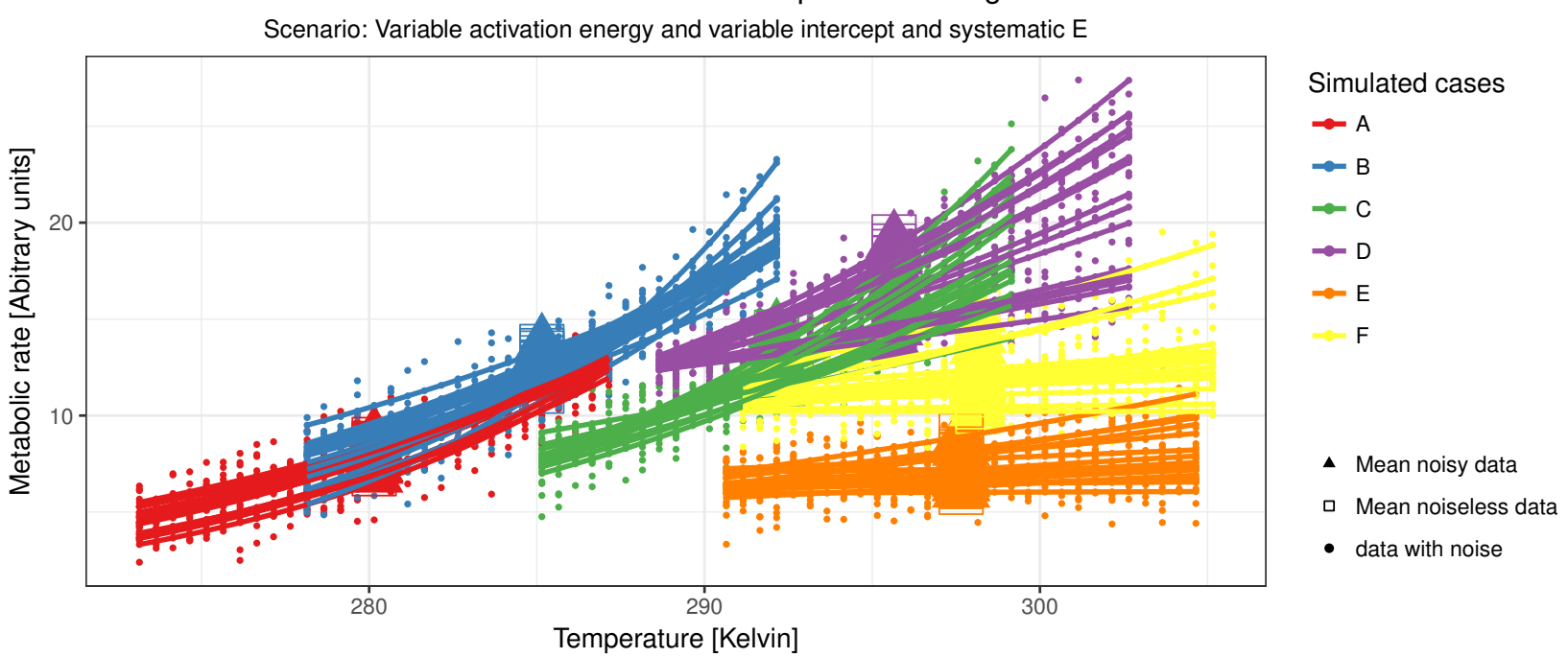

Log transformed simulated metabolic rates, averages and model predictions

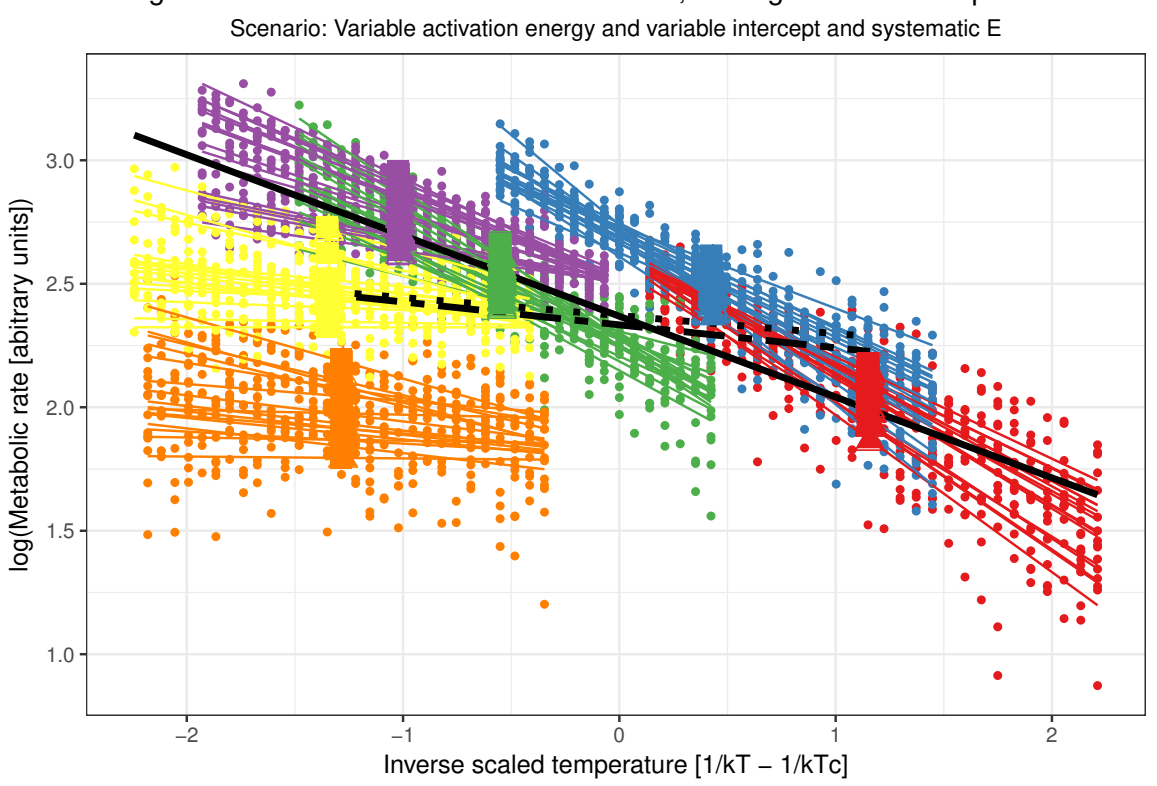

Simulated cases and Model predictions

A noiseless.mean_l_a

$\Delta$ noise.mean_I_a

noise.raw

Simulated cases and Model predictions

- Imer prediction - raw

-1 log first prediction

.... average first prediction 


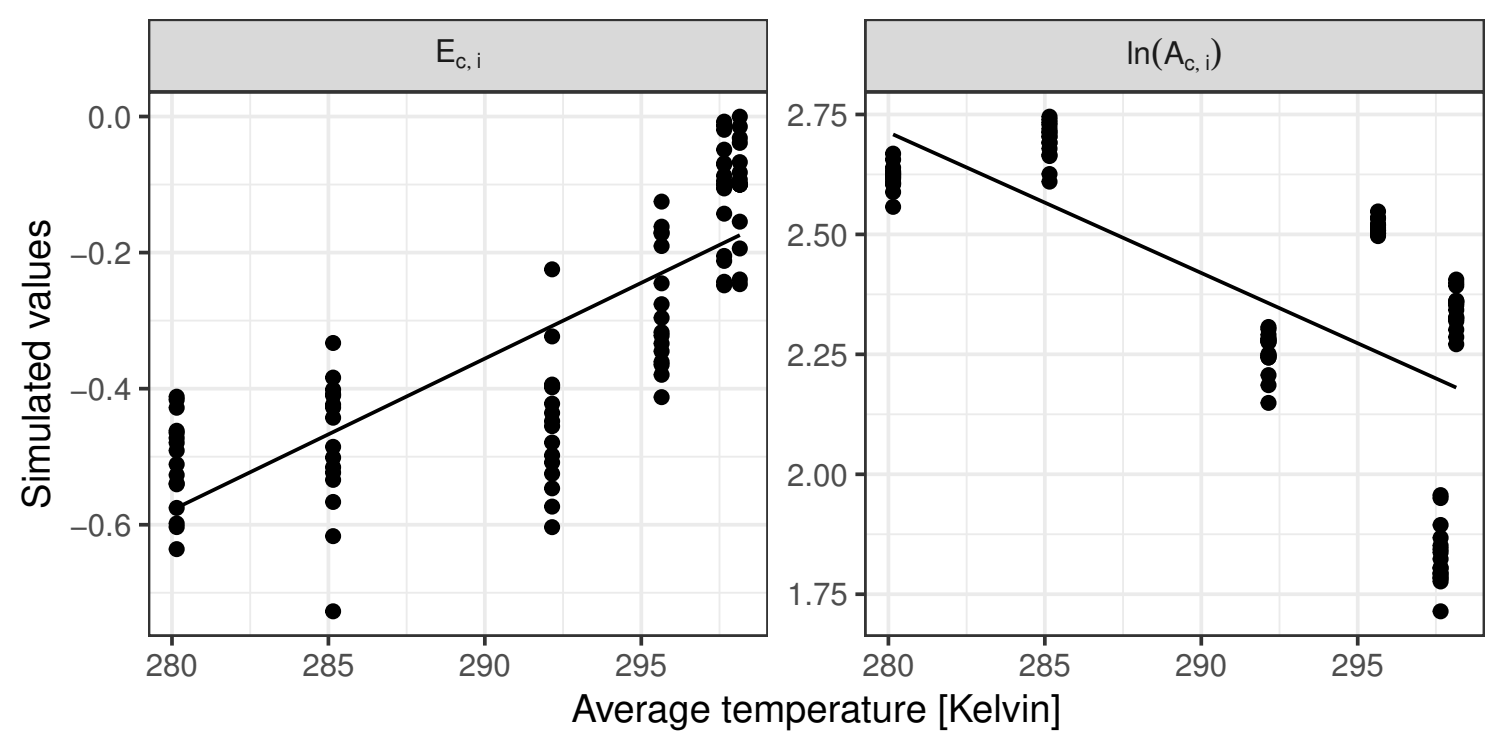

Figure 27: Scenario five: Regression of $\ln A_{c, i}$ and $E_{c, i}$ vs. $\overline{T_{c}}$ 


\subsection{Summary results scenario five:}

The estimates of $\hat{A}$ based on the LMER approach are almost exclusively determined by $\overline{\ln A_{c, i}}$ (see table 61 and figure 32 ). The estimates of $\hat{E}$ are solely determined by $\overline{E_{c, i}}$ (see table 59 and figure 32 ).

On the other hand, $\hat{E}$ estimated by the average approaches is largely influenced by $\beta_{A_{c, i}}$, i.e. the chance increases of $\overline{\ln A_{c, i}}$ with $\bar{T}_{c}$ and to a much smaller extent by $\overline{E_{c, i}}$ or $\beta_{E_{c, i}}$ the systematic increase of $\overline{E_{c, i}}$ with $\bar{T}_{c}$ (see tables 5155 and figures 30,31 ). $\hat{A}$ estimated by the average approaches is dominantly determined by $\overline{\ln A_{c, i}}$, still also the influence of $\beta_{A_{c, i}}$ is considerable (see tables 5357 and figures 30, 31). These difference between the 3 approaches are reflected in a much larger spread in $\overline{E_{c, i}}-\hat{E}$ and $\overline{\ln A_{c, i}}-\hat{A}$ for the average based approaches compared to the LMER approach (see figure 28) and the inability of the average based approaches to reproduce the distribution of $\overline{\ln A_{c, i}}$ or $\overline{E_{c, i}}$ (see figure 29). It seems clear that the LMER approach reproduces $\overline{E_{c, i}}$ faithfully, however the estimated average does not reveal that there is a systematic decrease of $E_{c}$ with increasing $\bar{T}_{c}$. It is arguable if $\overline{E_{c, i}}$ is of any interest, given that to understand changes in future temperature sensitivity of metabolic rates one would need to quantify the decrease in $\overline{E_{c, i}}$ with increasing $\bar{T}_{c}$. However, it is also clear that the average based approach are no alternative at all. The estimate $\hat{E}$ mainly seem to mirror chance correlation of $\overline{\ln A_{c, i}}$ with $\bar{T}_{c}$ and only to a very small extent $\overline{E_{c, i}}$ or even $\beta_{E_{c, i}}$.

\subsection{Scenario five: Comparison between $\overline{E_{c, i}}$ and $\overline{\ln A_{c, i}}$ and $\hat{E}$ and $\hat{A}$ from the 3 different estimation approaches}
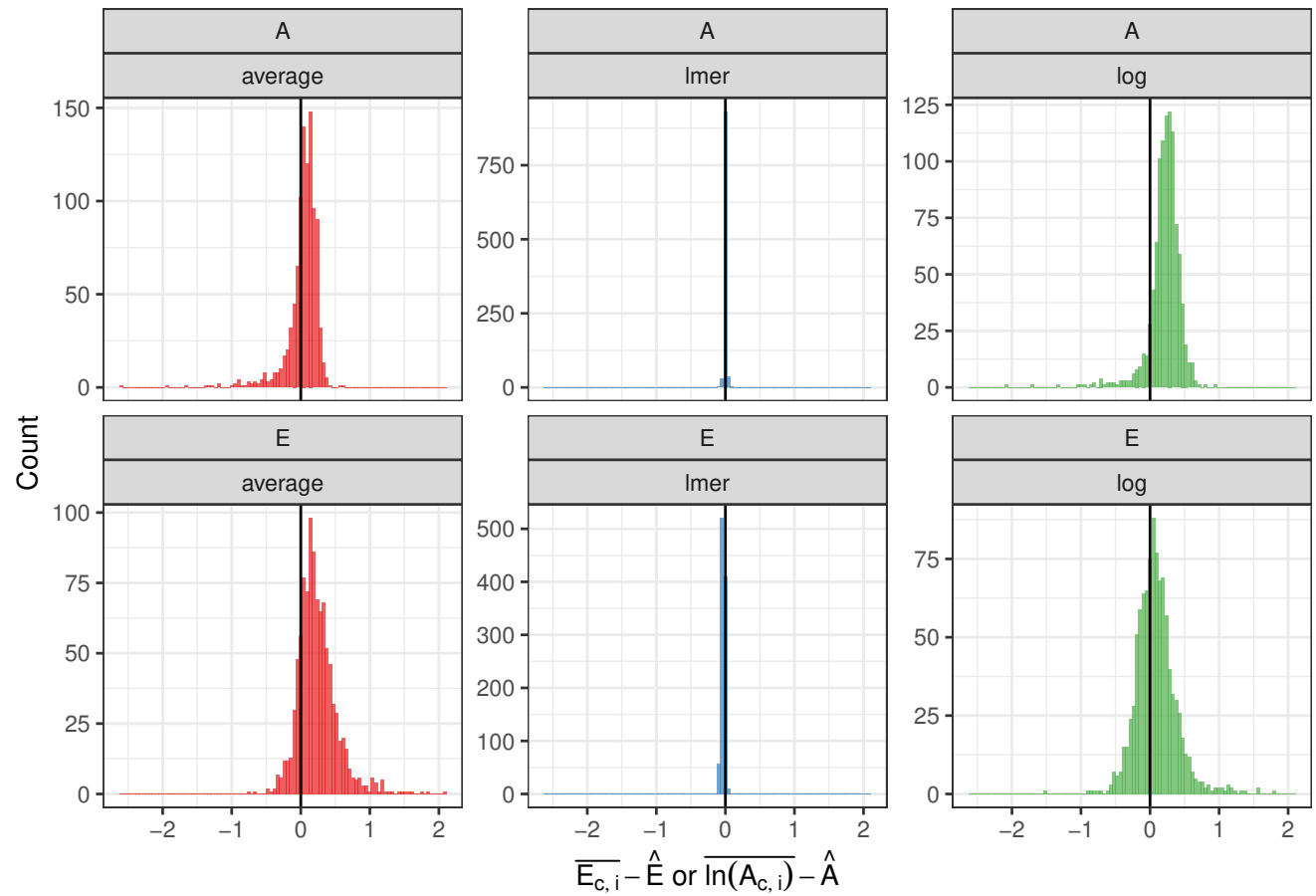

model

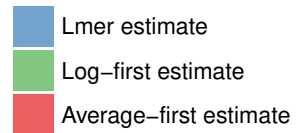

Average-first estimate

Figure 28: Scenario five: Comparison of $\overline{E_{c, i}}-\hat{E}$ and $\overline{\ln A_{c, i}}-\hat{A}$ for the 3 tested estimation approaches 

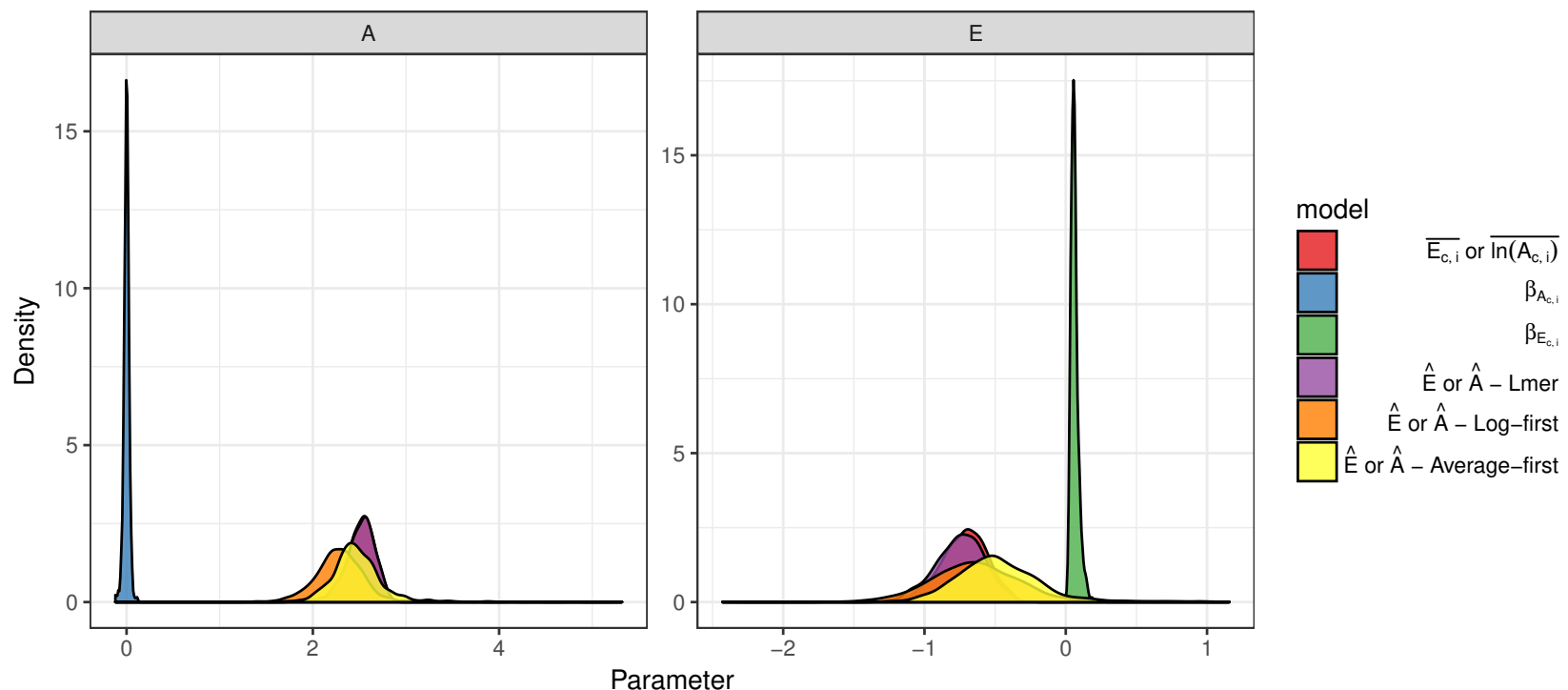

Figure 29: Scenario five: Comparison between simulated and estimated parameter 


\subsection{Scenario five: Detailed results on the influnece of chance correlation of}

$\ln A_{c, i} E_{c, i}$ with $\bar{T}_{c}$ on the estimates of $\hat{E}$ and $\hat{A}$

7.4.1 Log-first approach - Best model to explain $\hat{E}$ and $\hat{A}$ based on $\overline{E_{c, i}}, \overline{\ln A_{c, i}}, \beta_{E_{c, i}}$ and $\beta_{A_{c, i}}$
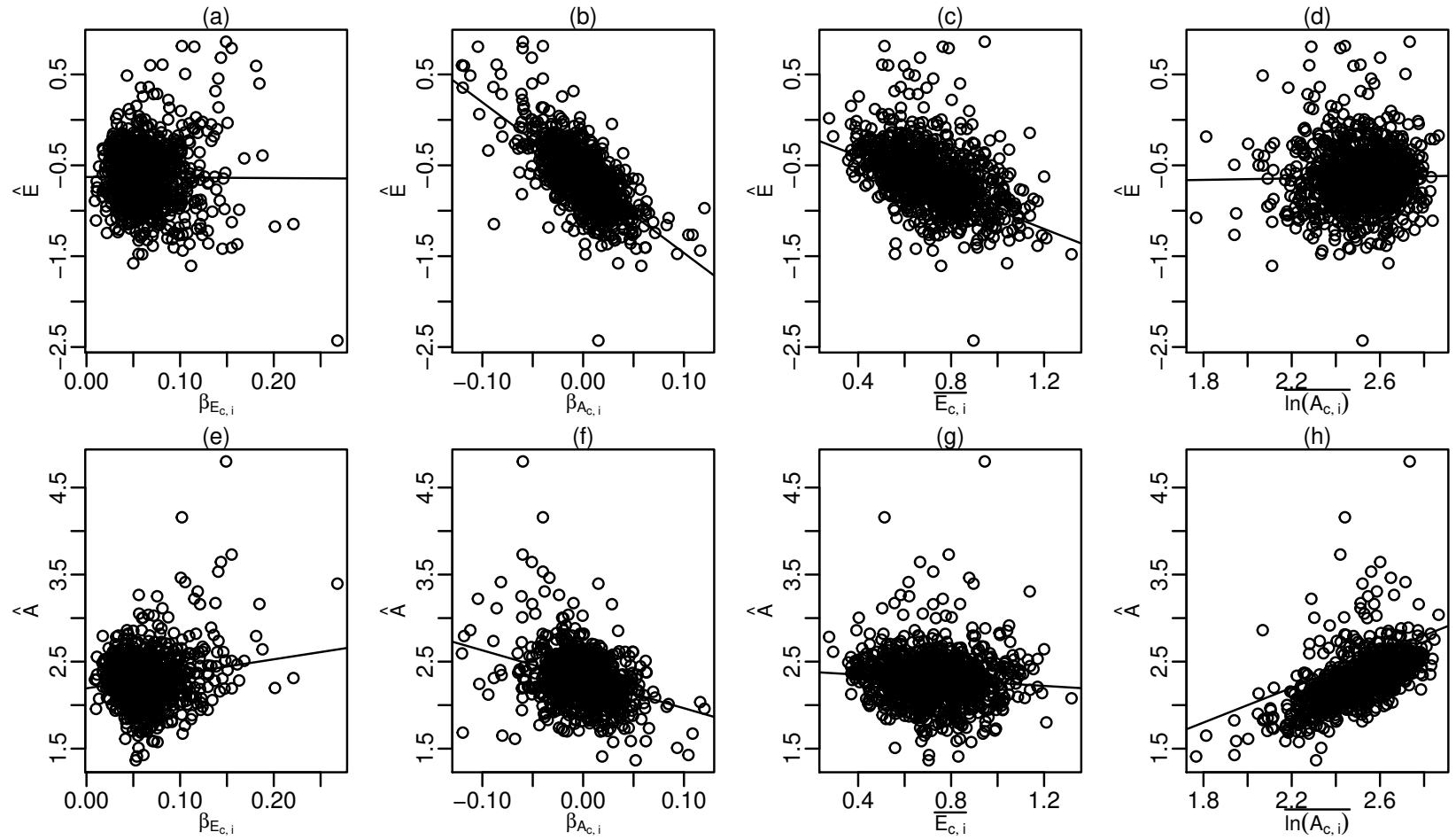

Figure 30: Scenario five - Log-first approach: Influence of $\beta_{E c, i}, \beta_{A c, i}, \overline{E_{c, i}}$, and $\overline{\ln A_{c, i}}$ on $\hat{E}$ and $\hat{A}$. Solid lines depict linear regression relations for all plot exept (c) and (h) were a bisection line is plotted since identity relations are expected.

Table 50: Scenario five - Log-first approach: Best model for $\hat{E}$

\begin{tabular}{lrrrr}
\hline & Estimate & Std. Error & t.value & $\operatorname{Pr}(>|t|)$ \\
\hline (Intercept) & 0.537 & 0.272 & 1.971 & 0.049 \\
$\overline{\ln A_{c, i}}$ & -0.171 & 0.106 & -1.619 & 0.106 \\
$\beta_{A_{c, i}}$ & -1.363 & 2.999 & -0.454 & 0.650 \\
$\beta_{E_{c, i}}$ & -10.731 & 4.103 & -2.615 & 0.009 \\
$\overline{E_{c, i}}$ & -1.088 & 0.092 & -11.887 & 0.000 \\
$\overline{\ln A_{c, i}}: \beta_{A_{c, i}}$ & -2.329 & 1.246 & -1.870 & 0.062 \\
$\ln A_{c, i}: \beta_{E_{c, i}}$ & 3.781 & 1.582 & 2.390 & 0.017 \\
$\beta_{A_{c, i}}: \beta_{E_{c, i}}$ & -15.593 & 6.241 & -2.498 & 0.013 \\
$\beta_{E_{c, i}}: \overline{E_{c, i}}$ & 2.469 & 1.356 & 1.820 & 0.069 \\
\hline
\end{tabular}

Table 51: Scenario five - Log-first approach: Relative importance of best model parameters to explain $\hat{E}$

\begin{tabular}{rr}
\hline & Rel. Importance \\
\hline $\ln A_{c, i}$ & 0.001
\end{tabular}




\begin{tabular}{lr}
\hline & Rel. Importance \\
\hline$\beta_{A_{c, i}}$ & 0.688 \\
$\frac{\beta_{E_{c, i}}}{\overline{E_{c, i}}}$ & 0.005 \\
$\overline{\ln A_{c, i}}: \beta_{A_{c, i}}$ & 0.297 \\
$\overline{\ln A_{c, i}: \beta_{E_{c, i}}}$ & 0.002 \\
$\beta_{A_{c, i}}: \beta_{E_{c, i}}$ & 0.002 \\
$\beta_{E_{c, i}}: E_{c, i}$ & 0.003 \\
\hline
\end{tabular}

Table 52: Scenario five - Log-first approach: Best model for $\hat{A}$

\begin{tabular}{lrrrr}
\hline & Estimate & Std. Error & t.value & $\operatorname{Pr}(>|t|)$ \\
\hline (Intercept) & 1.350 & 0.544 & 2.483 & 0.013 \\
$\frac{\ln A_{c, i}}{\beta_{A_{c, i}}}$ & 0.464 & 0.214 & 2.163 & 0.031 \\
$\beta_{E_{c, i}}$ & 3.486 & 3.243 & 1.075 & 0.283 \\
$\frac{E_{c, i}}{\ln A_{c, i}}: \beta_{A_{c, i}}$ & -1.207 & 1.143 & -1.056 & 0.291 \\
$\ln A_{c, i}: \overline{E_{c, i}}$ & -2.561 & 0.738 & -3.470 & 0.001 \\
$\beta_{A_{c, i}}: \beta_{E_{c, i}}$ & -2.057 & 1.349 & -1.525 & 0.127 \\
$\beta_{E_{c, i}}: \overline{E_{c, i}}$ & -25.528 & 0.291 & 2.933 & 0.003 \\
\hline & 3.403 & 1.467 & -3.833 & 0.000 \\
& & & &
\end{tabular}

Table 53: Scenario five - Log-first approach: Relative importance of best model parameters to explain $\hat{A}$

\begin{tabular}{lr}
\hline & Rel. Importance \\
$\overline{\overline{\ln A_{c, i}}}$ & 0.677 \\
$\beta_{A_{c, i}}$ & 0.215 \\
$\frac{\beta_{E_{c, i}}}{\overline{E_{c, i}}}$ & 0.051 \\
$\overline{\ln A_{c, i}}: \beta_{A_{c, i}}$ & 0.022 \\
$\overline{\ln A_{c, i}}: \overline{E_{c, i}}$ & 0.003 \\
$\beta_{A_{c, i}}: \beta_{E_{c, i}}$ & 0.010 \\
$\beta_{E_{c, i}}: \overline{E_{c, i}}$ & 0.016 \\
\hline
\end{tabular}


7.4.2 Average-first approach - Best model to explain $\hat{E}$ and $\hat{A}$ based on $\overline{E_{c, i}}, \overline{\ln A_{c, i}}, \beta_{E_{c, i}}$ and $\beta_{A_{c, i}}$
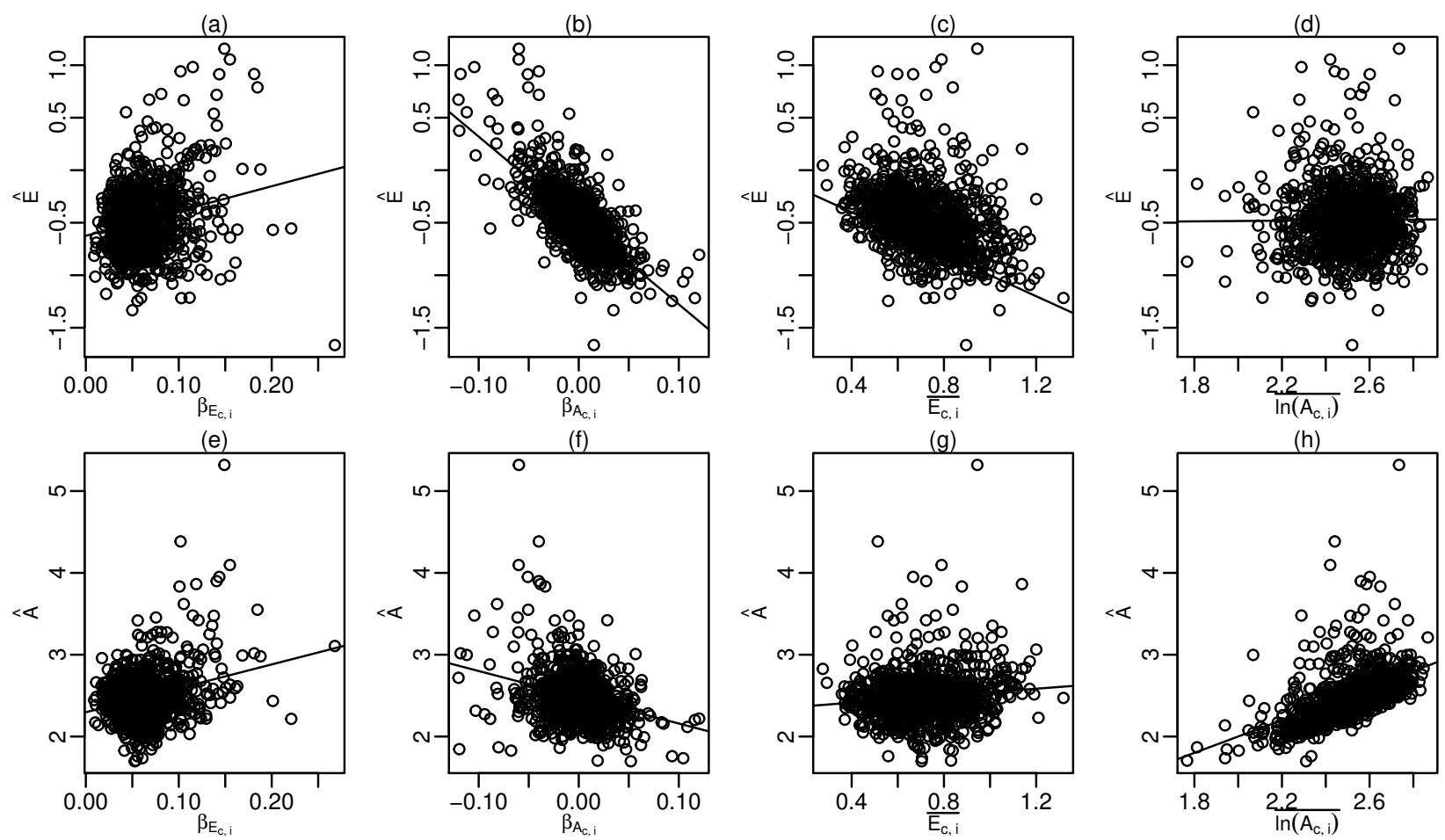

Figure 31: Scenario five - Average-first approach: Influence of $\beta_{E c, i}, \beta_{A c, i}, \overline{E_{c, i}}$, and $\overline{\ln A_{c, i}}$ on $\hat{E}$ and $\hat{A}$. Solid lines depict linear regression relations for all plot exept (c) and (h) were a bisection line is plotted since identity relations are expected.

Table 54: Scenario five - average-first approach: Best model for $\hat{E}$

\begin{tabular}{lrrrr}
\hline & Estimate & Std. Error & t.value & $\operatorname{Pr}(>|t|)$ \\
\hline (Intercept) & 0.529 & 0.242 & 2.184 & 0.029 \\
$\overline{\ln A_{c, i}}$ & -0.190 & 0.094 & -2.025 & 0.043 \\
$\beta_{A_{c, i}}$ & -0.036 & 2.668 & -0.013 & 0.989 \\
$\beta_{E_{c, i}}$ & -8.534 & 3.650 & -2.338 & 0.020 \\
$\overline{E_{c, i}}$ & -0.983 & 0.081 & -12.080 & 0.000 \\
$\overline{\ln A_{c, i}}: \beta_{A_{c, i}}$ & -2.728 & 1.108 & -2.462 & 0.014 \\
$\ln A_{c, i}: \beta_{E_{c, i}}$ & 3.427 & 1.408 & 2.435 & 0.015 \\
$\beta_{A_{c, i}}: \beta_{E_{c, i}}$ & -14.758 & 5.552 & -2.658 & 0.008 \\
$\beta_{E_{c, i}}: E_{c, i}$ & 3.660 & 1.207 & 3.033 & 0.002 \\
\hline
\end{tabular}

Table 55: Scenario five - average-first approach: Relative importance of best model parameters to explain $\hat{E}$

\begin{tabular}{lr}
\hline & Rel. Importance \\
\hline$\overline{\ln A_{c, i}}$ & 0.000 \\
$\beta_{A_{c, i}}$ & 0.707 \\
$\beta_{E_{c, i}}$ & 0.081
\end{tabular}




\begin{tabular}{lr}
\hline & Rel. Importance \\
$\overline{\overline{E_{c, i}}}: \beta_{A_{c, i}}$ & 0.201 \\
$\overline{\ln A_{c, i}}$ & 0.003 \\
$\overline{\ln A_{c, i}}: \beta_{E_{c, i}}$ & 0.002 \\
$\beta_{A_{c, i}}: \beta_{E_{c, i}}$ & 0.003 \\
$\beta_{E_{c, i}}: \overline{E_{c, i}}$ & 0.004 \\
\hline
\end{tabular}

Table 56: Scenario five - average-first approach: Best model for $\hat{A}$

\begin{tabular}{lrrrr}
\hline & Estimate & Std. Error & t.value & $\operatorname{Pr}(>|t|)$ \\
\hline (Intercept) & 1.470 & 0.577 & 2.550 & 0.011 \\
$\overline{\ln A_{c, i}}$ & 0.372 & 0.228 & 1.633 & 0.103 \\
$\beta_{A_{c, i}}$ & 6.485 & 3.314 & 1.956 & 0.051 \\
$\frac{\beta_{E_{c, i}}}{\overline{E_{c, i}}}$ & -10.350 & 4.647 & -2.227 & 0.026 \\
$\overline{\ln A_{c, i}}: \beta_{A_{c, i}}$ & -1.530 & 0.773 & -1.980 & 0.048 \\
$\overline{\ln A_{c, i}}: \beta_{E_{c, i}}$ & -3.190 & 1.377 & -2.316 & 0.021 \\
$\overline{\ln A_{c, i}}: \bar{E}_{c, i}$ & 3.917 & 1.789 & 2.189 & 0.029 \\
$\beta_{A_{c, i}}: \beta_{E_{c, i}}$ & -27.579 & 0.304 & 1.902 & 0.057 \\
$\beta_{E_{c, i}}: E_{c, i}$ & 3.461 & 6.902 & -4.012 & 0.000 \\
\hline
\end{tabular}

Table 57: Scenario five - average-first approach: Relative importance of best model parameters to explain $\hat{A}$

\begin{tabular}{lr}
\hline & Rel. Importance \\
\hline$\overline{\ln A_{c, i}}$ & 0.608 \\
$\beta_{A_{c, i}}$ & 0.196 \\
$\overline{\beta_{E_{c, i}}}$ & 0.137 \\
$\overline{E_{c, i}}$ & 0.019 \\
$\overline{\ln A_{c, i}}: \beta_{A_{c, i}}$ & 0.007 \\
$\overline{\ln A_{c, i}}: \beta_{E_{c, i}}$ & 0.005 \\
$\overline{\ln A_{c, i}}: \bar{E}_{c, i}$ & 0.005 \\
$\beta_{A_{c, i}}: \beta_{E_{c, i}}$ & 0.017 \\
$\beta_{E_{c, i}}: E_{c, i}$ & 0.005 \\
\hline
\end{tabular}


7.4.3 LMER approach - Best model to explain $\hat{E}$ and $\hat{A}$ based on $\overline{E_{c, i}}, \overline{\ln A_{c, i}}, \beta_{E_{c, i}}$ and $\beta_{A_{c, i}}$
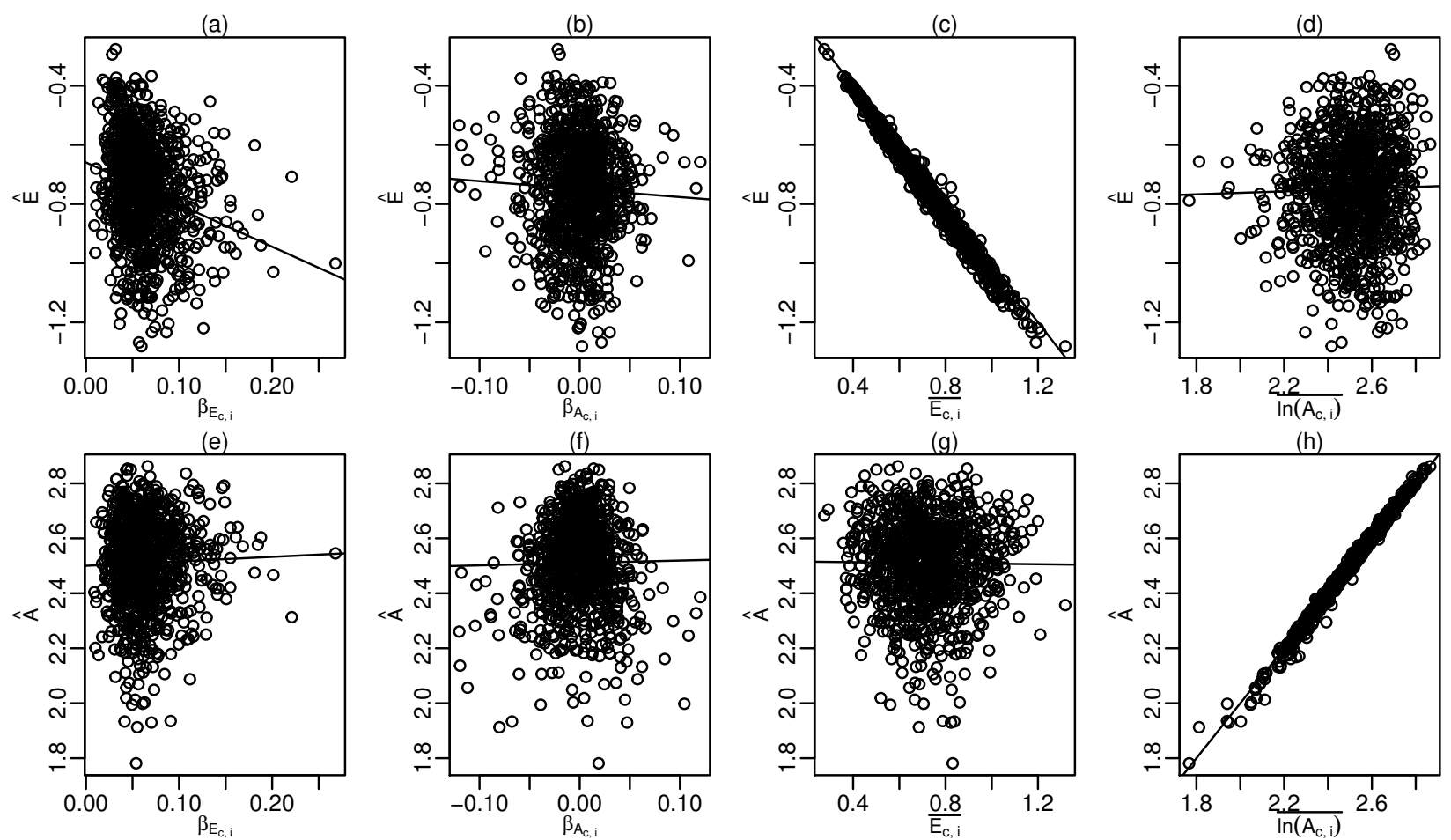

Figure 32: Scenario five - LMER approach: Influence of $\beta_{E c, i}, \beta_{A c, i}, \overline{E_{c, i}}$, and $\overline{\ln A_{c, i}}$ on $\hat{E}$ and $\hat{A}$. Solid lines depict linear regression relations for all plot exept (c) and (h) were a bisection line is plotted since identity relations are expected.

Table 58: Scenario five - lmer approach: Best model for $\hat{E}$

\begin{tabular}{lrrrr}
\hline & Estimate & Std. Error & t.value & $\operatorname{Pr}(>|t|)$ \\
\hline (Intercept) & -0.030 & 0.012 & -2.564 & 0.010 \\
$\overline{\ln A_{c, i}}$ & 0.013 & 0.004 & 2.996 & 0.003 \\
$\beta_{A_{c, i}}$ & 1.660 & 0.350 & 4.735 & 0.000 \\
$\beta_{E_{c, i}}$ & 0.041 & 0.025 & 1.663 & 0.097 \\
$\overline{E_{c, i}}$ & -1.053 & 0.004 & -239.409 & 0.000 \\
$\overline{\ln A_{c, i}}: \beta_{A_{c, i}}$ & -0.811 & 0.138 & -5.879 & 0.000 \\
$\beta_{A_{c, i}}: \overline{E_{c, i}}$ & 0.274 & 0.169 & 1.625 & 0.104 \\
\hline
\end{tabular}

Table 59: Scenario five - lmer approach: Relative importance of best model parameters to explain $\hat{E}$

\begin{tabular}{lr}
\hline & Rel. Importance \\
\hline$\overline{\overline{\ln A_{c, i}}}$ & 0.000 \\
$\beta_{A_{c, i}}$ & 0.001 \\
$\frac{\beta_{E_{c, i}}}{E_{c, i}}$ & 0.021 \\
$\overline{\ln A_{c, i}}: \beta_{A_{c, i}}$ & 0.976 \\
$\beta_{A_{c, i}}: \overline{E_{c, i}}$ & 0.001 \\
\hline
\end{tabular}


Table 60: Scenario five - lmer approach: Best model for $\hat{A}$

\begin{tabular}{lrrrr}
\hline & Estimate & Std. Error & t.value & $\operatorname{Pr}(>|t|)$ \\
\hline (Intercept) & -0.085 & 0.008 & -10.261 & 0.000 \\
$\frac{\ln A_{c, i}}{\beta_{A_{c, i}}}$ & 1.030 & 0.003 & 376.800 & 0.000 \\
$\frac{\beta_{E_{c, i}}}{E_{c, i}}$ & -0.458 & 0.215 & -2.132 & 0.033 \\
$\frac{\ln A_{c, i}}{\beta_{A_{c, i}}: \beta_{A_{c, i}}}: \overline{E_{c, i}}$ & 0.101 & 0.072 & 1.415 & 0.157 \\
$\beta_{E_{c, i}}: \overline{E_{c, i}}$ & 0.018 & 0.006 & 2.909 & 0.004 \\
\hline
\end{tabular}

Table 61: Scenario five - lmer approach: Relative importance of best model parameters to explain $\hat{A}$

\begin{tabular}{lr}
\hline & Rel. Importance \\
\hline$\overline{\ln A_{c, i}}$ & 0.999 \\
$\beta_{A_{c, i}}$ & 0.000 \\
$\beta_{E_{c, i}}$ & 0.001 \\
$\overline{E_{c, i}}$ & 0.000 \\
$\overline{\ln A_{c, i}}: \beta_{A_{c, i}}$ & 0.000 \\
$\beta_{A_{c, i}}: \overline{E_{c, i}}$ & 0.000 \\
$\beta_{E_{c, i}}: \overline{E_{c, i}}$ & 0.000 \\
\hline
\end{tabular}




\section{Scenario six: All entities have variale $A_{c}$ and $E_{c}$. $A_{c}$ 's systemat- ically increase with increasing $\bar{T}_{c}$}

\subsection{Description and assumptions of scenario six}

In this scenario $A_{c}$ and $E_{c}$ are both randomly drawn and thus are different for all entities. It is, however, assumed that the $A_{c}$ systematically increases with $\bar{T}_{c}$, while $E_{c}$ can only exhibit chance correlation with $\bar{T}_{c}$ (see figure 33). Thus, while under this assumption the estimation of $\overline{E_{c, i}}$ is of interest, this is not necessarily the case for $\overline{\ln A_{c, i}}$. Rather in the light of a systematic temperature dependence of the absolute metabolic rates the qualification and quantification of $\beta_{A_{c, i}}$ would be of core interest.

Simulated metabolic rates and respective averages

Scenario: Variable activation energy and variable intercept and systematic $\mathrm{A}$

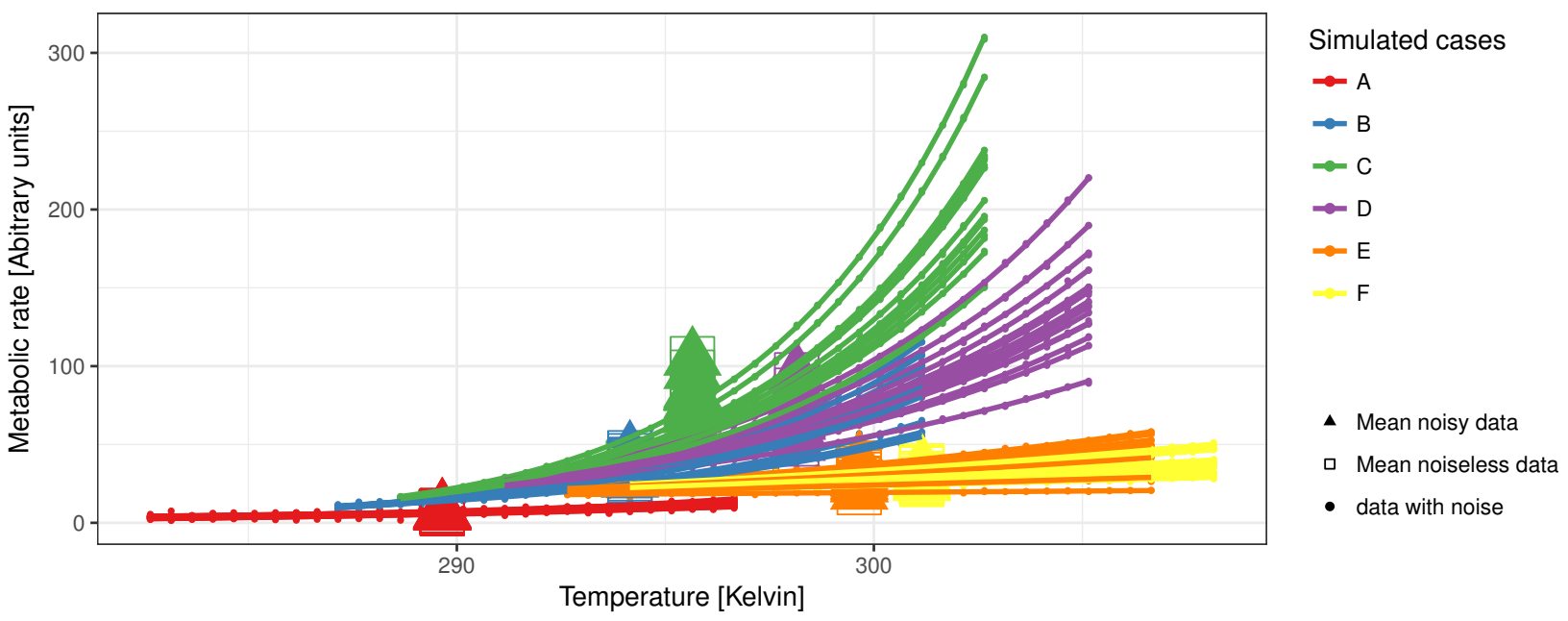

Log transformed simulated metabolic rates, averages and model predictions Scenario: Variable activation energy and variable intercept and systematic $A$

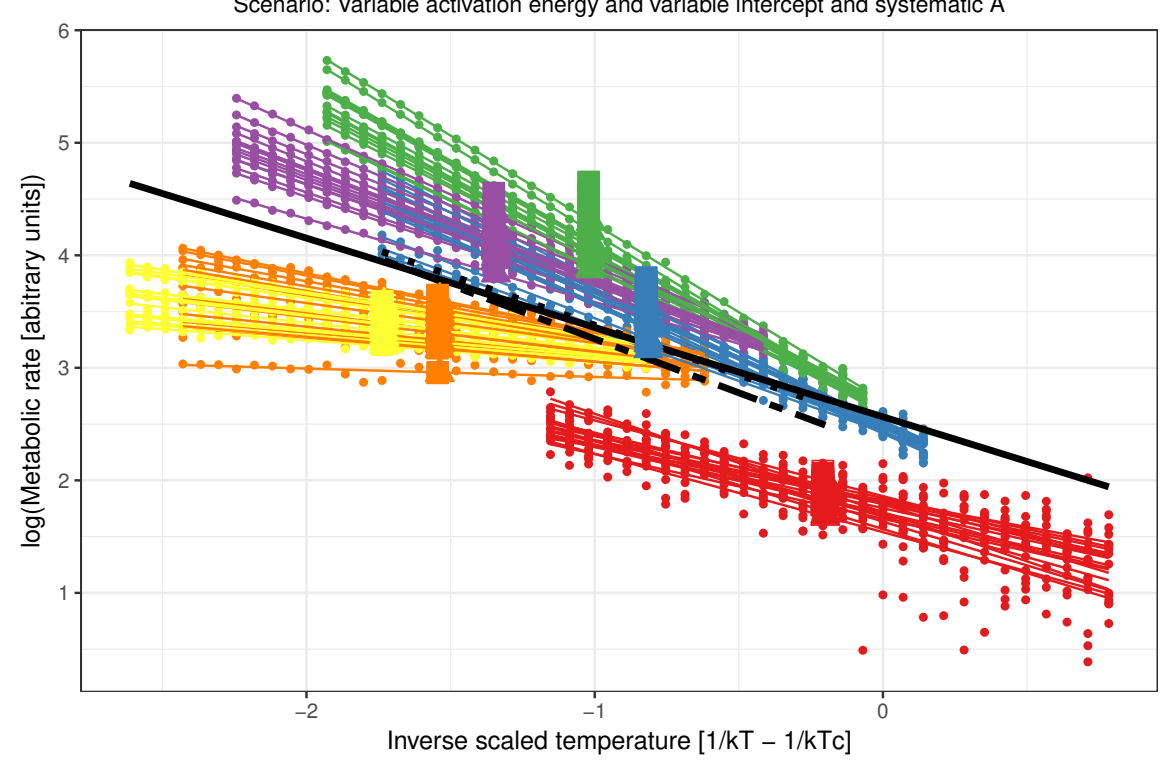

Simulated cases and Model predictions

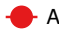

$B$

C $C$

- D

- E

F

noiseless.mean a

回 noise.mean_a

$\Delta$ noiseless.mean__aa

$\triangle$ noise.mean_l_a

noise.raw

Simulated cases and Model predictions

- Imer prediction - raw

- $\mathbf{l}$ log first prediction

.... average first prediction 


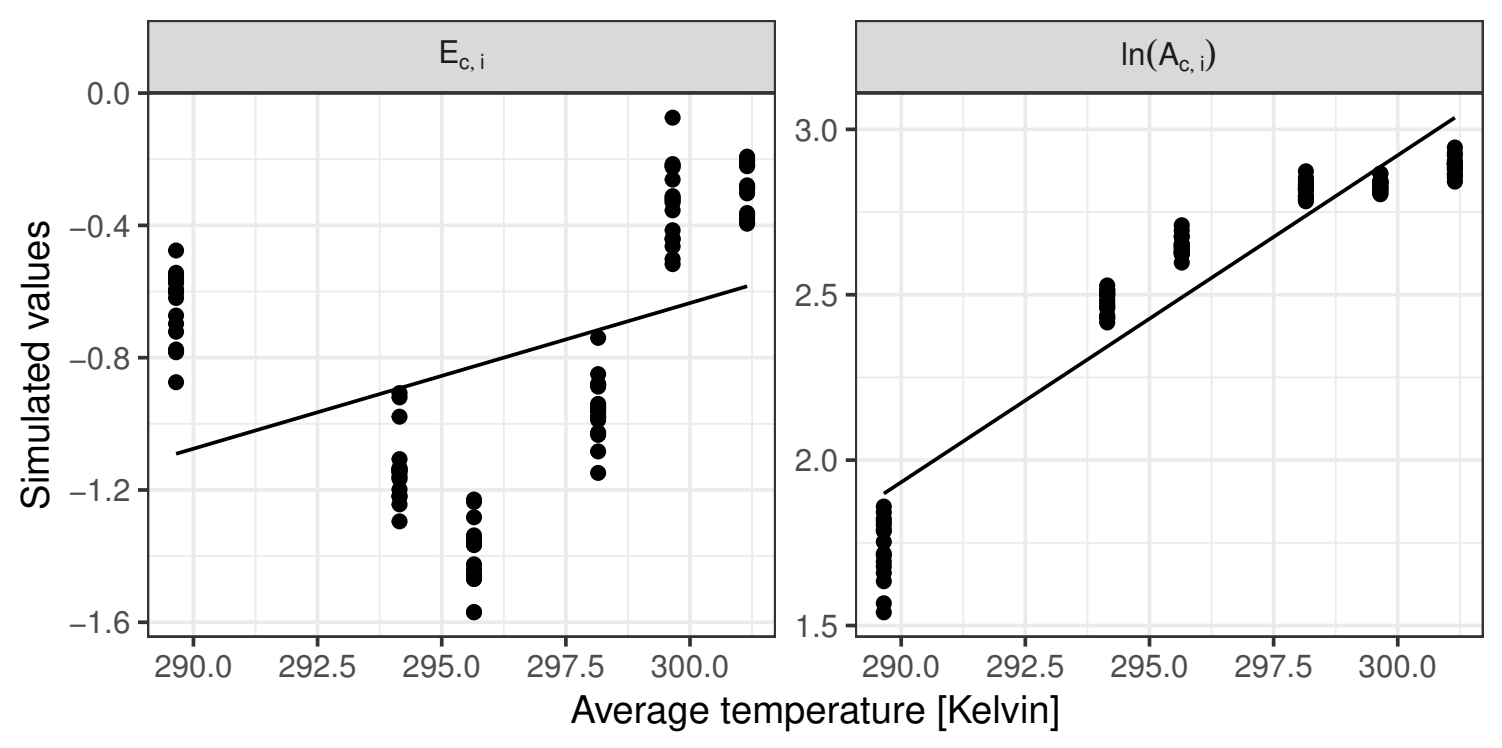

Figure 33: Scenario six: Regression of $\ln A_{c, i}$ and $E_{c, i}$ vs. $\overline{T_{c}}$ 


\subsection{Summary results scenario six:}

$\hat{E}$ estimated by the LMER approach are almost exclusively determined by $\overline{E_{c, i}}$ (see table71 and figure 38 ) and $\hat{A}$ by $\overline{\ln A_{c, i}}$, yet to a smaller amount also by $\beta_{A_{c, i}}$ (see table73 and figure 38 ). $\hat{E}$ estimated by either of the average based approaches in mainly influenced by $\beta_{A_{c, i}}$ followed by $\overline{E_{c, i}}$ (see tables 63,67 and figures $36,37)$. The most impotent factors determining the estimates of $\hat{A}$ based on either of the average based approaches are $\overline{\ln A_{c, i}}$ followed by $\beta_{A_{c, i}}$ (see tables 65,69 and figures 36,37 ).

The differences between simulated and average values of $\overline{\ln A_{c, i}}-\hat{A}$ and $\overline{E_{c, i}}-\hat{E}$ have a large variance for the average based approaches, which is in sharp contrast to the LMER approach with very little variance. In addition estimates of $\overline{E_{c, i}}$ by both average approaches seem on average substantially overestimated (see figure 34). This is in line with a considerable deviation of the density distribution of the simulated parameters compared to the estimated ones based on the average based approaches (see figure 35).

Thus, while the LMER approach estimates average values well, the estimates by the average based approaches are influenced by both the average parameter value but also by $\beta_{A_{c, i}}$, thus they neither reproduce the average nor the systematic changes in a systematic way.

\subsection{Scenario six: Comparison between $\overline{E_{c, i}}$ and $\overline{\ln A_{c, i}}$ and $\hat{E}$ and $\hat{A}$ from the 3 different estimation approaches}
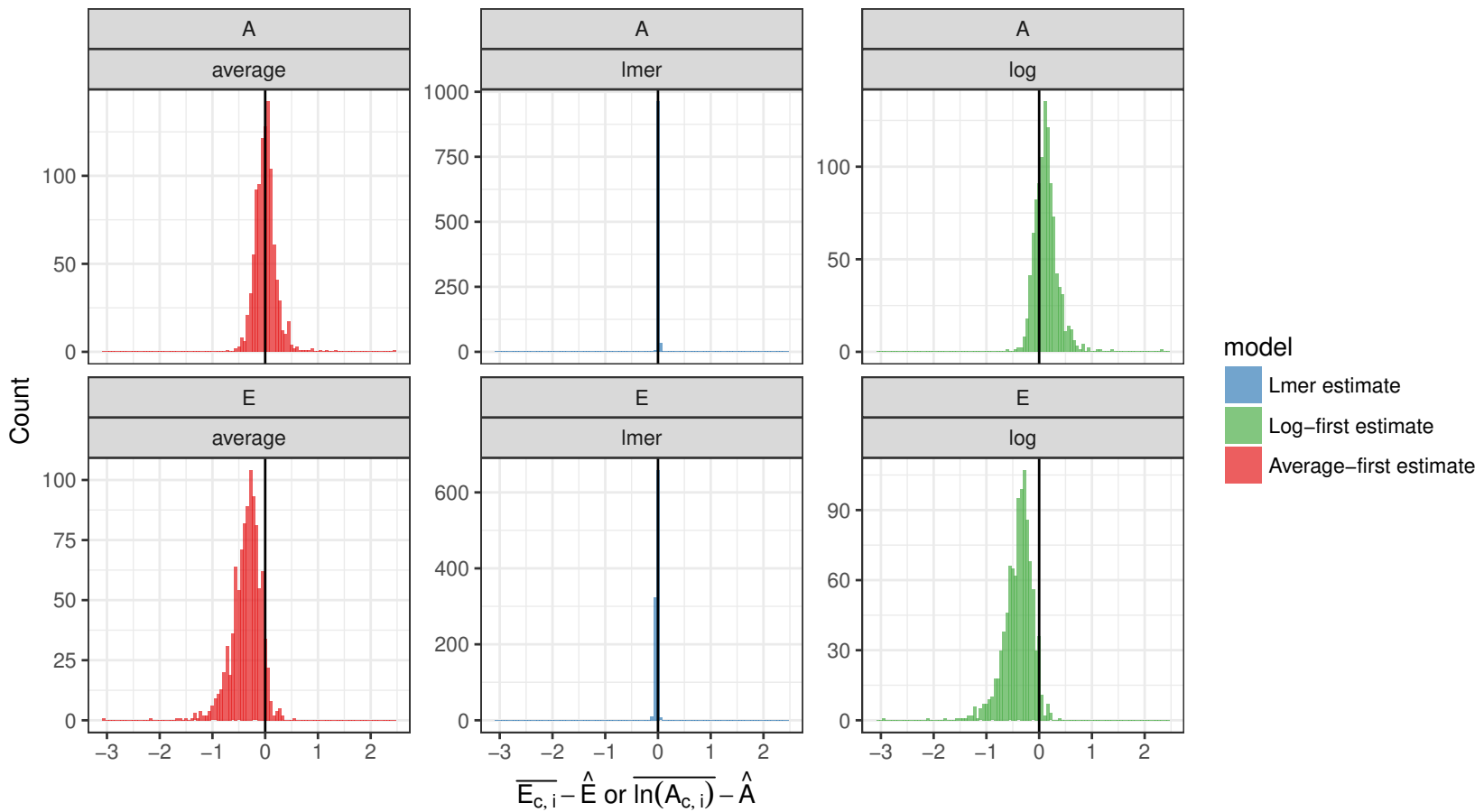

Figure 34: Scenario six: Comparison of $\overline{E_{c, i}}-\hat{E}$ and $\overline{\ln A_{c, i}}-\hat{A}$ for the 3 tested estimation approaches 


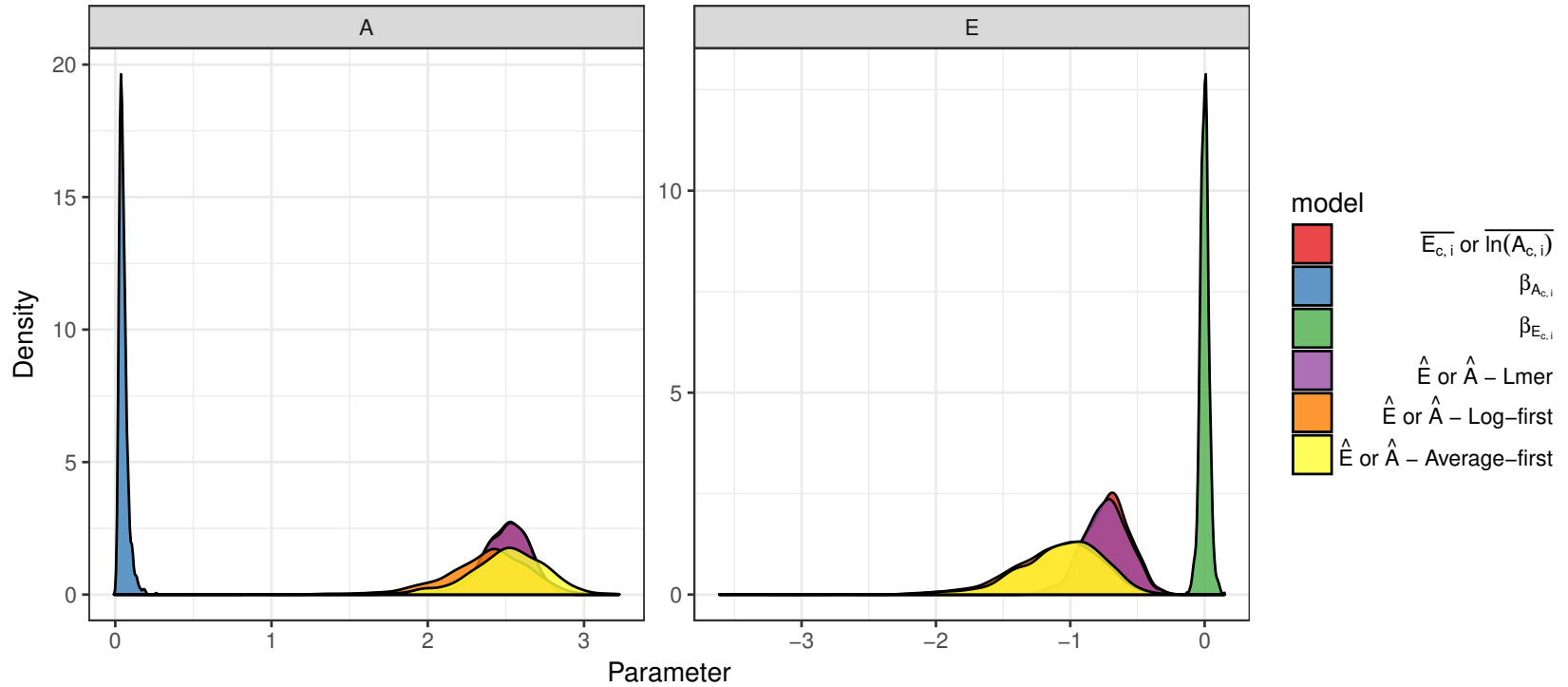

Figure 35: Scenario six: Comparison between simulated and estimated parameter 


\subsection{Scenario six: Detailed results on the influnece of chance correlation of $\ln A_{c, i}$ $E_{c, i}$ with $\bar{T}_{c}$ on the estimates of $\hat{E}$ and $\hat{A}$}

8.4.1 Log-first approach - Best model to explain $\hat{E}$ and $\hat{A}$ based on $\overline{E_{c, i}}, \overline{\ln A_{c, i}}, \beta_{E_{c, i}}$ and $\beta_{A_{c, i}}$
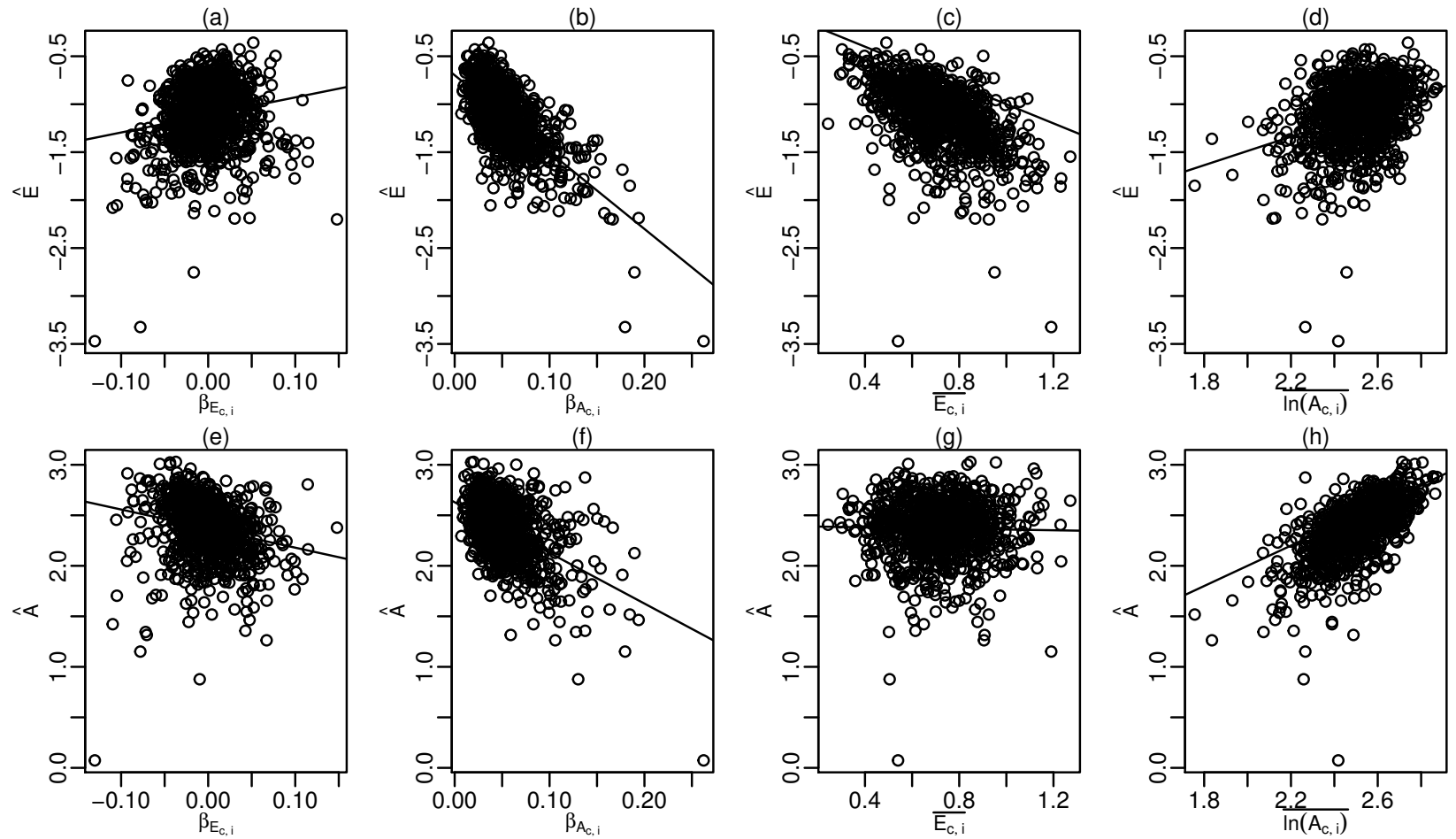

Figure 36: Scenario six - Log-first approach: Influence of $\beta_{E c, i}, \beta_{A c, i}, \overline{E_{c, i}}$, and $\overline{\ln A_{c, i}}$ on $\hat{E}$ and $\hat{A}$. Solid lines depict linear regression relations for all plot exept (c) and (h) were a bisection line is plotted since identity relations are expected.

Table 62: Scenario six - Log-first approach: Best model for $\hat{E}$

\begin{tabular}{lrrrr}
\hline & Estimate & Std. Error & t.value & $\operatorname{Pr}(>|t|)$ \\
\hline (Intercept) & -0.216 & 0.117 & -1.841 & 0.066 \\
\hline $\ln A_{c, i}$ & 0.086 & 0.044 & 1.974 & 0.049 \\
$\beta_{A_{c, i}}$ & -7.443 & 0.229 & -32.504 & 0.000 \\
$\beta_{E_{c, i}}$ & 1.062 & 0.326 & 3.255 & 0.001 \\
\hline$E_{c, i}$ & -1.017 & 0.035 & -28.851 & 0.000 \\
$\beta_{A_{c, i}}: \beta_{E_{c, i}}$ & 6.715 & 4.208 & 1.596 & 0.111 \\
\hline
\end{tabular}

Table 63: Scenario six - Log-first approach: Relative importance of best model parameters to explain $\hat{E}$

\begin{tabular}{lr}
\hline & Rel. Importance \\
\hline$\overline{\ln A_{c, i}}$ & 0.055 \\
$\beta_{A_{c, i}}$ & 0.556 \\
$\beta_{E_{c, i}}$ & 0.037 \\
\hline$E_{c, i}$ & 0.351
\end{tabular}




\begin{tabular}{lr}
\hline & Rel. Importance \\
\hline$\beta_{A_{c, i}}: \beta_{E_{c, i}}$ & 0.001 \\
\hline
\end{tabular}

Table 64: Scenario six - Log-first approach: Best model for $\hat{A}$

\begin{tabular}{lrrrr}
\hline & Estimate & Std. Error & t.value & $\operatorname{Pr}(>|t|)$ \\
\hline (Intercept) & 0.855 & 0.525 & 1.630 & 0.103 \\
$\overline{\ln A_{c, i}}$ & 0.705 & 0.199 & 3.538 & 0.000 \\
$\beta_{A_{c, i}}$ & -4.406 & 1.005 & -4.383 & 0.000 \\
$\beta_{E_{c, i}}$ & -18.507 & 3.652 & -5.067 & 0.000 \\
$\overline{E_{c, i}}$ & -1.443 & 0.726 & -1.989 & 0.047 \\
$\overline{\ln A_{c, i}}: \beta_{E_{c, i}}$ & 4.470 & 1.371 & 3.261 & 0.001 \\
$\ln A_{c, i}: \overline{E_{c, i}}$ & 0.502 & 0.276 & 1.823 & 0.069 \\
$\beta_{A_{c, i}}: \beta_{E_{c, i}}$ & 56.063 & 4.932 & 11.367 & 0.000 \\
$\beta_{A_{c, i}}: \overline{E_{c, i}}$ & 2.910 & 1.370 & 2.124 & 0.034 \\
$\beta_{E_{c, i}}: E_{c, i}$ & 2.020 & 1.072 & 1.884 & 0.060 \\
\hline
\end{tabular}

Table 65: Scenario six - Log-first approach: Relative importance of best model parameters to explain $\hat{A}$

\begin{tabular}{lr}
\hline & Rel. Importance \\
\hline$\overline{\ln A_{c, i}}$ & 0.540 \\
$\beta_{A_{c, i}}$ & 0.258 \\
$\overline{\beta_{E_{c, i}}}$ & 0.093 \\
$\overline{E_{c, i}}$ & 0.001 \\
$\overline{\ln A_{c, i}}: \beta_{E_{c, i}}$ & 0.006 \\
$\overline{\ln A_{c, i}}: \overline{E_{c, i}}$ & 0.002 \\
$\beta_{A_{c, i}}: \beta_{E_{c, i}}$ & 0.092 \\
$\beta_{A_{c, i}}: \overline{E_{c, i}}$ & 0.006 \\
$\beta_{E_{c, i}}: \overline{E_{c, i}}$ & 0.002 \\
\hline
\end{tabular}


8.4.2 Average-first approach - Best model to explain $\hat{E}$ and $\hat{A}$ based on $\overline{E_{c, i}}, \overline{\ln A_{c, i}}, \beta_{E_{c, i}}$ and $\beta_{A_{c, i}}$
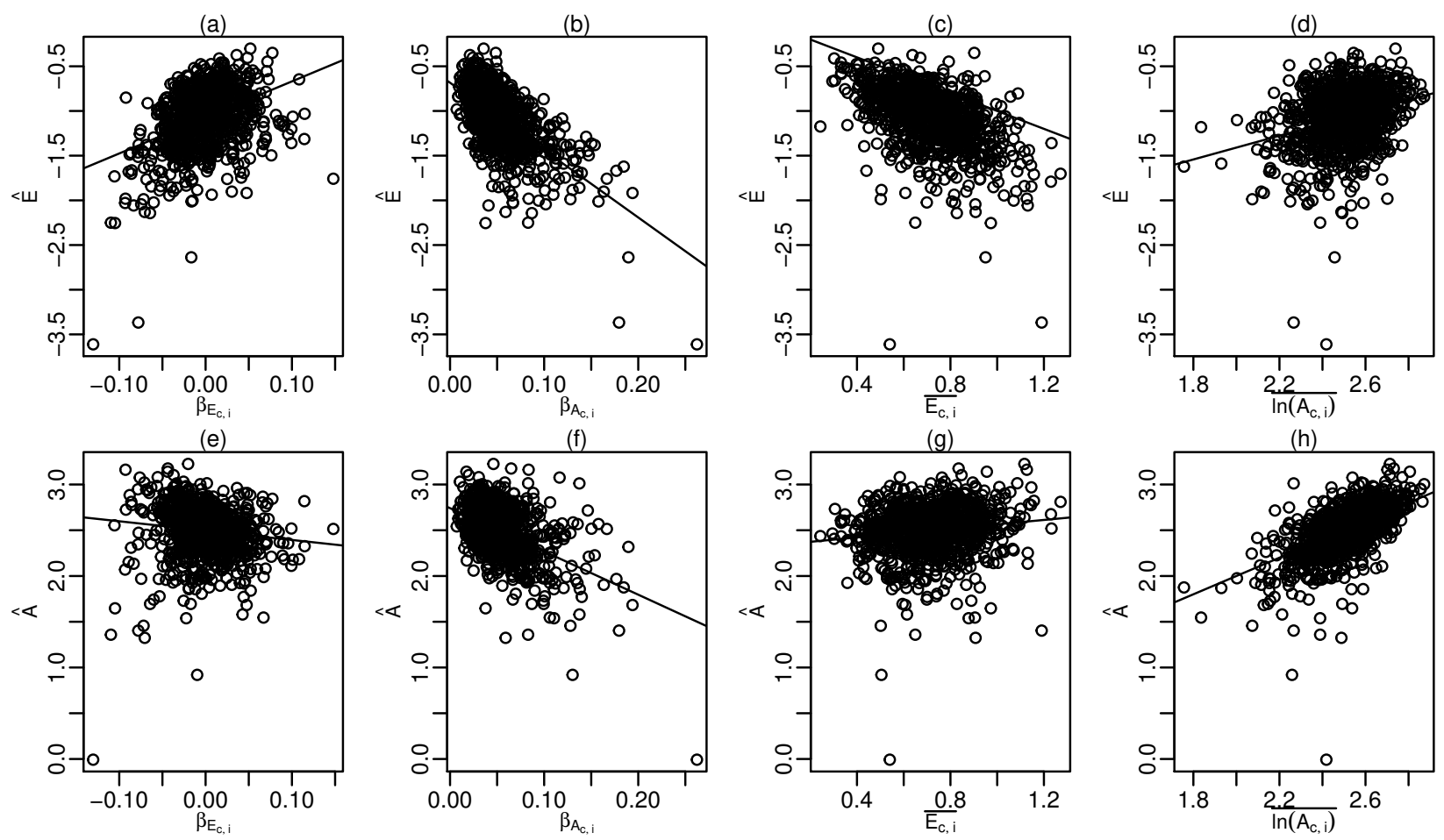

Figure 37: Scenario six - Average-first approach: Influence of $\beta_{E c, i}, \beta_{A c, i}, \overline{E_{c, i}}$, and $\overline{\ln A_{c, i}}$ on $\hat{E}$ and $\hat{A}$. Solid lines depict linear regression relations for all plot exept (c) and (h) were a bisection line is plotted since identity relations are expected.

Table 66: Scenario six - average-first approach: Best model for $\hat{E}$

\begin{tabular}{lrrrr}
\hline & Estimate & Std. Error & t.value & $\operatorname{Pr}(>|t|)$ \\
\hline (Intercept) & -0.027 & 0.025 & -1.058 & 0.290 \\
$\beta_{A_{c, i}}$ & -7.111 & 0.189 & -37.544 & 0.000 \\
$\beta_{E_{c, i}}$ & 2.047 & 0.789 & 2.593 & 0.010 \\
\hline$E_{c, i}$ & -0.948 & 0.033 & -28.861 & 0.000 \\
$\beta_{A_{c, i}}: \beta_{E_{c, i}}$ & 6.319 & 3.895 & 1.622 & 0.105 \\
$\beta_{E_{c, i}}: E_{c, i}$ & 1.672 & 0.952 & 1.756 & 0.079 \\
\hline
\end{tabular}

Table 67: Scenario six - average-first approach: Relative importance of best model parameters to explain $\hat{E}$

\begin{tabular}{lr}
\hline & Rel. Importance \\
\hline$\beta_{A_{c, i}}$ & 0.502 \\
$\beta_{E_{c, i}}$ & 0.195 \\
\hline$E_{c, i}$ & 0.301 \\
$\beta_{A_{c, i}}: \beta_{E_{c, i}}$ & 0.001 \\
$\beta_{E_{c, i}}: E_{c, i}$ & 0.001 \\
\hline
\end{tabular}


Table 68: Scenario six - average-first approach: Best model for $\hat{A}$

\begin{tabular}{lrrrr}
\hline & Estimate & Std. Error & t.value & $\operatorname{Pr}(>|t|)$ \\
\hline (Intercept) & 1.090 & 0.533 & 2.047 & 0.041 \\
$\ln A_{c, i}$ & 0.586 & 0.202 & 2.896 & 0.004 \\
$\beta_{A_{c, i}}$ & -4.554 & 1.020 & -4.464 & 0.000 \\
$\frac{\beta_{E_{c, i}}}{\overline{E_{c, i}}}$ & -16.090 & 3.706 & -4.341 & 0.000 \\
$\overline{\ln A_{c, i}}: \beta_{E_{c, i}}$ & -1.376 & 0.737 & -1.868 & 0.062 \\
$\overline{\ln A_{c, i}}: \bar{E}_{c, i}$ & 3.806 & 1.391 & 2.737 & 0.006 \\
$\beta_{A_{c, i}}: \beta_{E_{c, i}}$ & 0.580 & 0.280 & 2.075 & 0.038 \\
$\beta_{A_{c, i}}: \overline{E_{c, i}}$ & 32.194 & 5.005 & 10.429 & 0.000 \\
$\beta_{E_{c, i}}: \overline{E_{c, i}}$ & 2.226 & 1.390 & 2.321 & 0.021 \\
\hline
\end{tabular}

Table 69: Scenario six - average-first approach: Relative importance of best model parameters to explain $\hat{A}$

\begin{tabular}{lr}
\hline & Rel. Importance \\
$\overline{\overline{\ln A_{c, i}}}$ & 0.550 \\
$\beta_{A_{c, i}}$ & 0.264 \\
$\frac{\beta_{E_{c, i}}}{E_{c, i}}$ & 0.034 \\
$\overline{\ln A_{c, i}}: \beta_{E_{c, i}}$ & 0.038 \\
$\overline{\ln A_{c, i}}: \overline{E_{c, i}}$ & 0.006 \\
$\beta_{A_{c, i}}: \beta_{E_{c, i}}$ & 0.003 \\
$\beta_{A_{c, i}}: \overline{E_{c, i}}$ & 0.093 \\
$\beta_{E_{c, i}}: E_{c, i}$ & 0.007 \\
\hline
\end{tabular}


8.4.3 LMER approach - Best model to explain $\hat{E}$ and $\hat{A}$ based on $\overline{E_{c, i}}, \overline{\ln A_{c, i}}, \beta_{E_{c, i}}$ and $\beta_{A_{c, i}}$
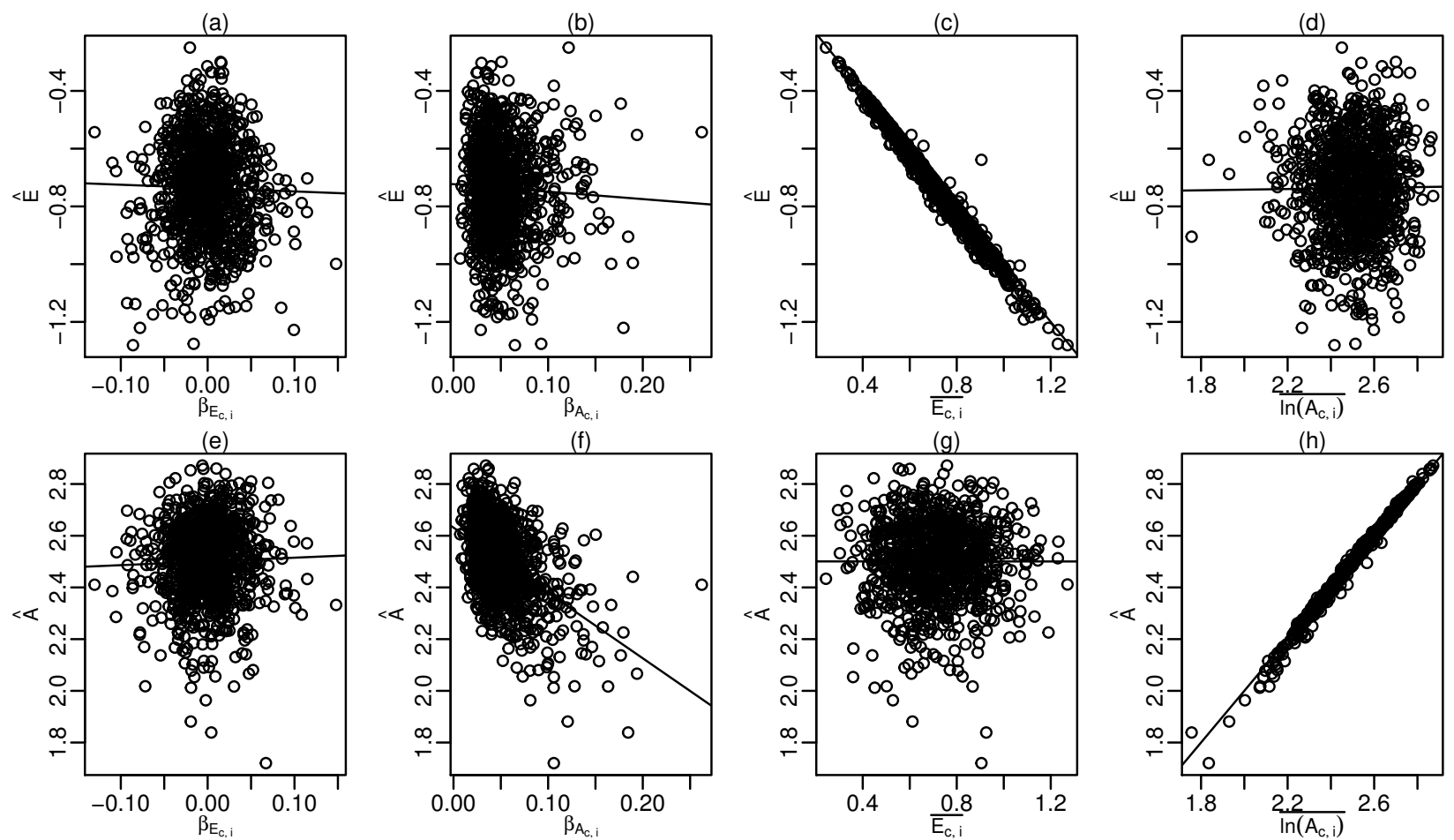

Figure 38: Scenario six - LMER approach: Influence of $\beta_{E c, i}, \beta_{A c, i}, \overline{E_{c, i}}$, and $\overline{\ln A_{c, i}}$ on $\hat{E}$ and $\hat{A}$. Solid lines depict linear regression relations for all plot exept (c) and (h) were a bisection line is plotted since identity relations are expected.

Table 70: Scenario six - lmer approach: Best model for $\hat{E}$

\begin{tabular}{lrrrr}
\hline & Estimate & Std. Error & t.value & $\operatorname{Pr}(>|t|)$ \\
\hline (Intercept) & -0.287 & 0.053 & -5.444 & 0.000 \\
$\frac{\ln A_{c, i}}{\beta_{A_{c, i}}}$ & 0.115 & 0.021 & 5.471 & 0.000 \\
$\frac{\beta_{E_{c, i}}}{E_{c, i}}$ & 1.647 & 0.300 & 5.486 & 0.000 \\
$\overline{\ln A_{c, i}}: \beta_{A_{c, i}}$ & -0.885 & 0.416 & 2.129 & 0.033 \\
$\overline{\ln A_{c, i}}: \beta_{E_{c, i}}$ & -0.839 & 0.071 & -11.848 & 0.000 \\
$\ln A_{c, i}: \overline{E_{c, i}}$ & -0.072 & 0.124 & -5.301 & 0.000 \\
$\beta_{A_{c, i}}: \beta_{E_{c, i}}$ & 1.025 & 0.156 & -3.352 & 0.001 \\
$\beta_{E_{c, i}}: \overline{E_{c, i}}$ & 0.199 & 0.028 & -2.749 & 0.006 \\
\hline
\end{tabular}

Table 71: Scenario six - lmer approach: Relative importance of best model parameters to explain $\hat{E}$

\begin{tabular}{lr}
\hline & Rel. Importance \\
\hline$\overline{\ln A_{c, i}}$ & 0.000 \\
$\beta_{A_{c, i}}$ & 0.001 \\
$\beta_{E_{c, i}}$ & 0.001
\end{tabular}




\begin{tabular}{lr}
\hline & Rel. Importance \\
$\overline{\overline{E_{c, i}}}: \beta_{A_{c, i}}$ & 0.997 \\
$\overline{\ln A_{c, i}}: \beta_{E_{c, i}}$ & 0.001 \\
$\overline{\ln A_{c, i}}: \overline{E_{c, i}}$ & 0.000 \\
$\beta_{A_{c, i}}: \beta_{E_{c, i}}$ & 0.000 \\
$\beta_{E_{c, i}}: \overline{E_{c, i}}$ & 0.000 \\
\hline
\end{tabular}

Table 72: Scenario six - lmer approach: Best model for $\hat{A}$

\begin{tabular}{lrrrr}
\hline & Estimate & Std. Error & t.value & $\operatorname{Pr}(>|t|)$ \\
\hline (Intercept) & -0.026 & 0.012 & -2.099 & 0.036 \\
$\frac{\ln A_{c, i}}{\beta_{A_{c, i}}}$ & 1.012 & 0.005 & 209.513 & 0.000 \\
$\frac{\beta_{E_{c, i}}}{E_{c, i}}$ & -0.642 & 0.166 & -3.864 & 0.000 \\
$\frac{\ln A_{c, i}}{\ln A_{c, i}: \beta_{A_{c, i}}}$ & -0.851 & 0.195 & -4.356 & 0.000 \\
$\beta_{E_{c, i}}: \bar{E}_{c, i}$ & -0.002 & 0.002 & -1.077 & 0.282 \\
\hline
\end{tabular}

Table 73: Scenario six - lmer approach: Relative importance of best model parameters to explain $\hat{A}$

\begin{tabular}{lr}
\hline & Rel. Importance \\
\hline$\overline{\overline{\ln A_{c, i}}}$ & 0.904 \\
$\beta_{A_{c, i}}$ & 0.095 \\
$\overline{\beta_{E_{c, i}}}$ & 0.000 \\
$\overline{E_{c, i}}$ & 0.000 \\
$\overline{\ln A_{c, i}}: \beta_{A_{c, i}}$ & 0.000 \\
$\overline{\ln A_{c, i}}: \beta_{E_{c, i}}$ & 0.000 \\
\hline$\beta_{E_{c, i}}: \bar{E}_{c, i}$ & 0.000 \\
\hline
\end{tabular}




\section{Scenario seven: All entities are allowed to have variable $A_{c}$ and $E_{c}$ which systematically increase respectively decrease with $\bar{T}$}

\subsection{Description and assumptions of scenario seven}

In this scenario randomly drawn $A_{c}$ and $E_{c}$ are forced to systematically increase respectively decrease with increasing $\bar{T}_{c}$ (see figure 39 ).

The temperature dependent changes in $E_{c}$ and $A_{c}$ raise the question if estimates of $\overline{\ln A_{c, i}}$ and $\overline{E_{c, i}}$ are of particular interest, or if rather the qualification and quantification of the increase of $E_{c}=\beta_{E c} \cdot \bar{T}_{c}$ and $A_{c}=\beta_{A c} \cdot \bar{T}_{c}$ are in the focus of interest particular under a climate change aspect.

Simulated metabolic rates and respective averages

Scenario: Variable activation energy and variable intercept and systematic $A$ and $E$

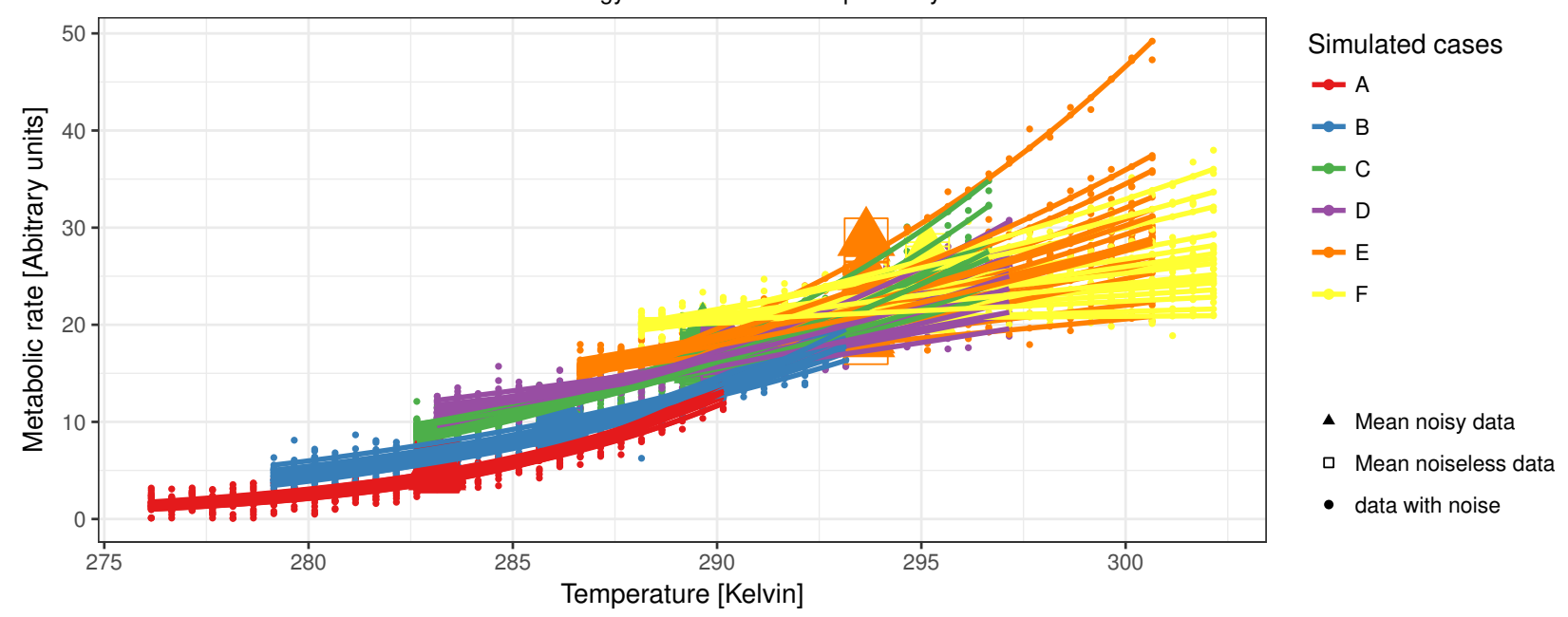

Log transformed simulated metabolic rates, averages and model predictions Scenario: Variable activation energy and variable intercept and systematic $A$ and $E$

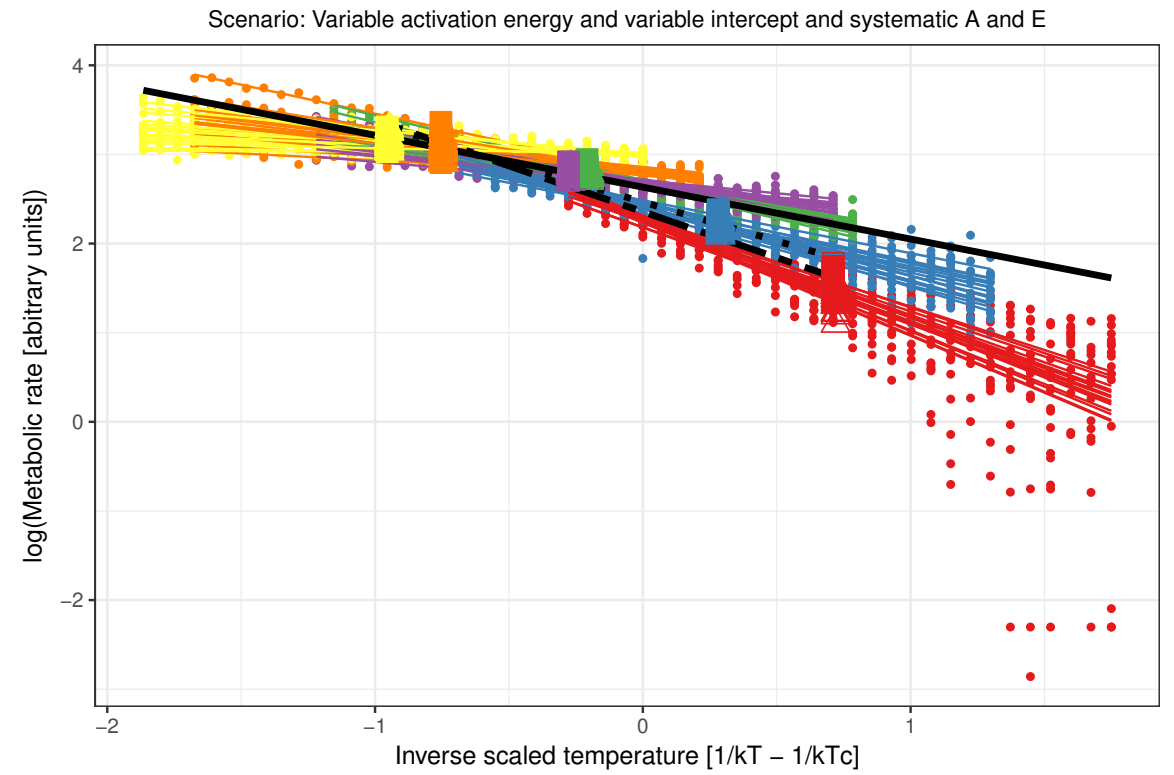

Simulated cases and Model predictions

- A

B

C

- D

E

$\mathrm{F}$

noiseless.mean_a_l

回 noise.mean_a_।

$\boldsymbol{\Delta}$ noiseless.mean_I_a

$\Delta$ noise.mean_l_a

- noise.raw

Simulated cases and Model predictions

- Imer prediction - raw

- $\log$ first prediction

.... average first prediction 


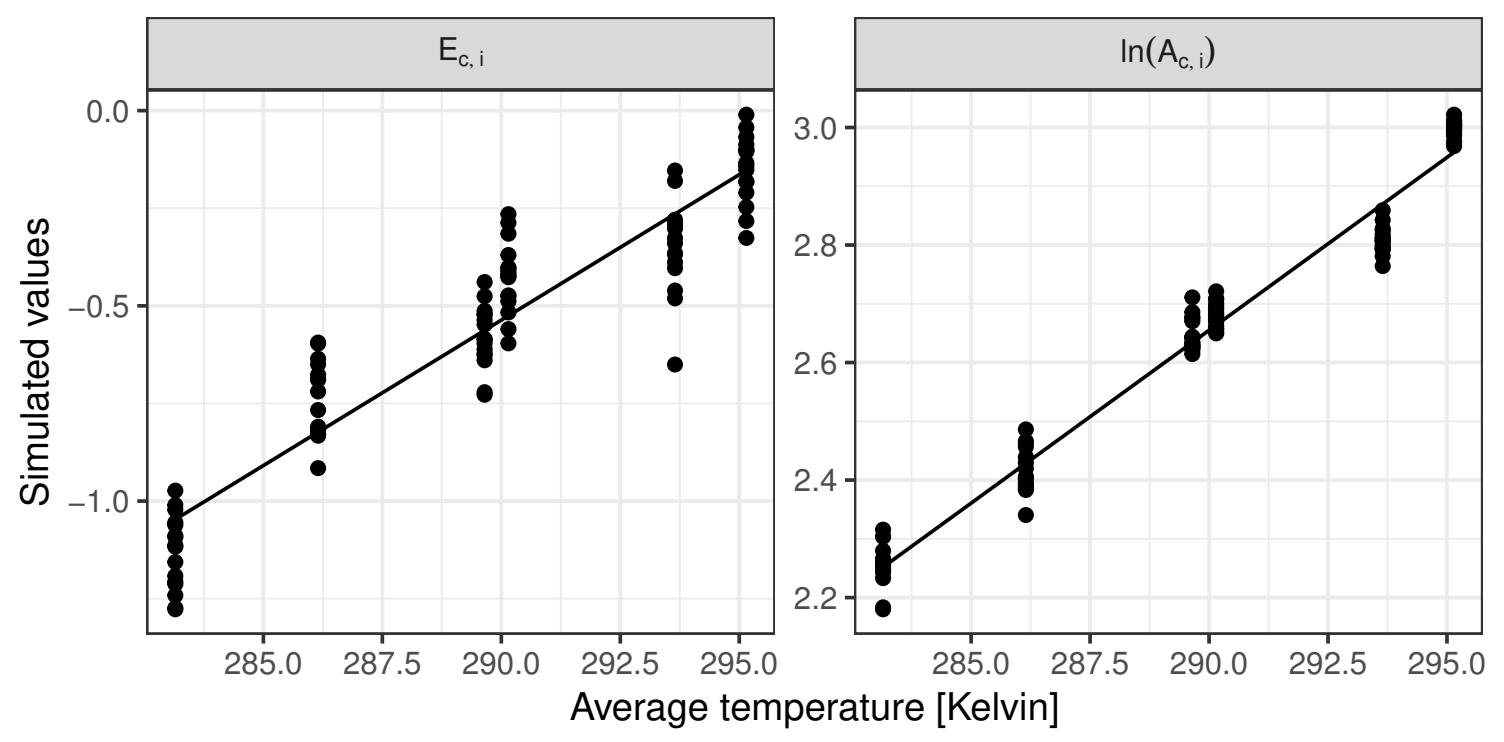

Figure 39: Scenario seven: Regression of $\ln A_{c, i}$ and $E_{c, i}$ vs. $\overline{T_{c}}$ 


\subsection{Summary results scenario seven:}

$\hat{E}$ estimated by the LMER approach is predominately determined by $\overline{E_{c, i}}$, while the systematic increases of these averages, quantified in $\beta_{A_{c, i}}$ and $\beta_{E_{c, i}}$, only have very minor relevance (see table 83 and figure 44 ). Similar, $\hat{A}$ estimated by the LMER approach is mainly determined by $\overline{\ln A_{c, i}}$ and to a lesser extend also by $\beta_{A_{c, i}}$ (see table85 and figure 44). Differences between simulated and estimated values, i.e. $\overline{\ln A_{c, i}}-\hat{A}$ and $\overline{E_{c, i}}-\hat{E}$ are closely centered around zero (see figure 40 ) and estimated values resemble well the density distribution. $\hat{E}$, however, seems on average to be slightly overestimated (see figure 41).

In comparison the estimates of $\hat{E}$ is almost as much influenced by $\overline{E_{c, i}}$ than by $\beta_{A_{c, i}}$ this holds for both average approaches (see tables 75,79 and figures 42,43 ). $\hat{A}$ estimated by the average approaches is determined $\overline{\ln A_{c, i}}$ followed by $\beta_{A_{c, i}}$ and for the average-first approach also $\beta_{E_{c, i}}$ (see tables 77, 77 and figures 42, 43). Both differences, $\overline{\ln A_{c, i}}-\hat{A}$ and $\overline{E_{c, i}}-\hat{E}$, vary considerably for the average based approach. Both approaches tend to under estimate $\overline{\ln A_{c, i}}$ but overestimate $\overline{E_{c, i}}$ considerably (see figures 40 and 41 ). In summary the LMER approach is the only one which reasonably produces average estimates and is rather insensitive to the systematic changes, on the other hand the average based approaches are sensitive to the systematic increases but are unable to quantify them in a useful way.

\subsection{Scenario seven: Comparison between $\overline{E_{c, i}}$ and $\overline{\ln A_{c, i}}$ and $\hat{E}$ and $\hat{A}$ from the 3 different estimation approaches}
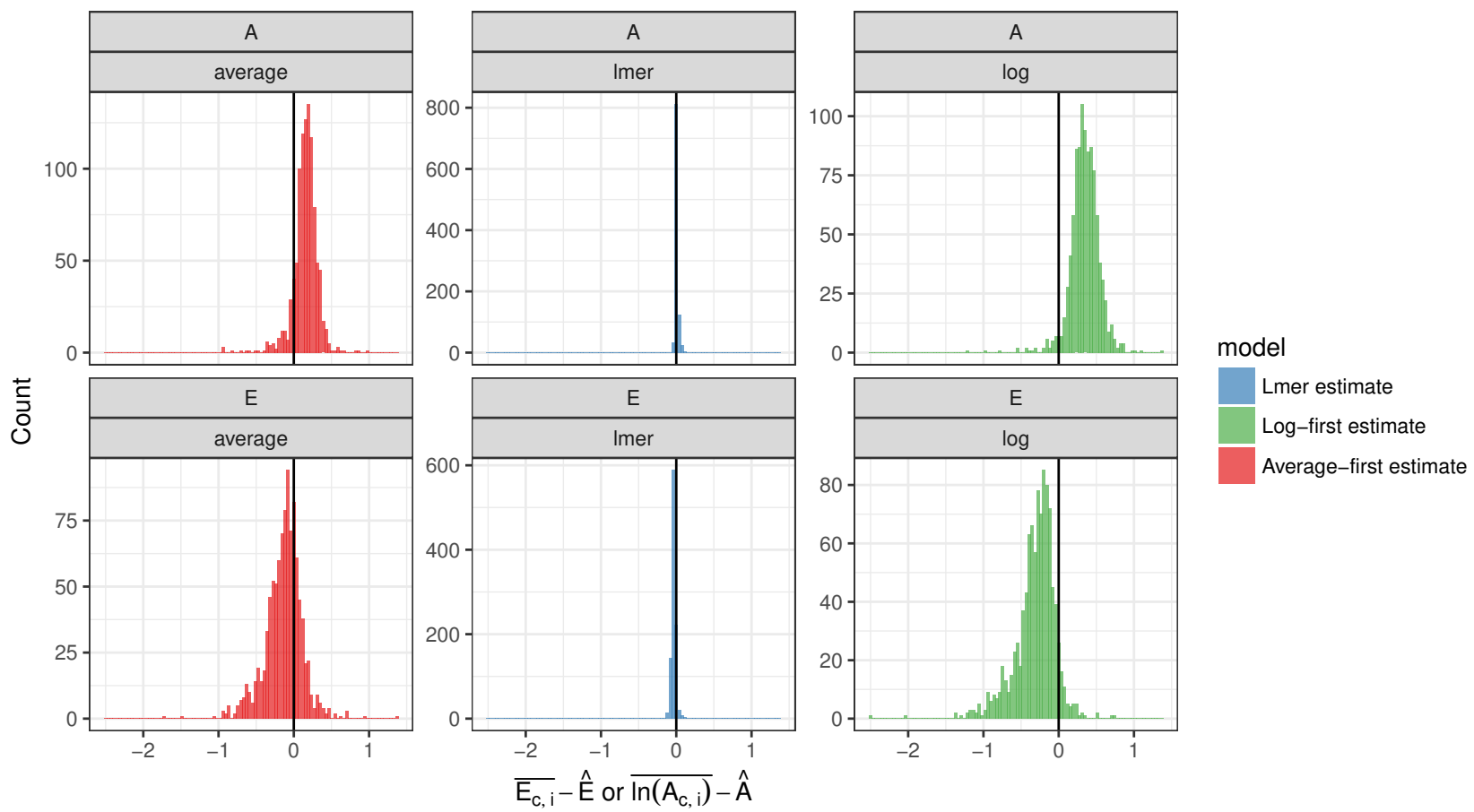

Figure 40: Scenario seven: Comparison of $\overline{E_{c, i}}-\hat{E}$ and $\overline{\ln A_{c, i}}-\hat{A}$ for the 3 tested estimation approaches 

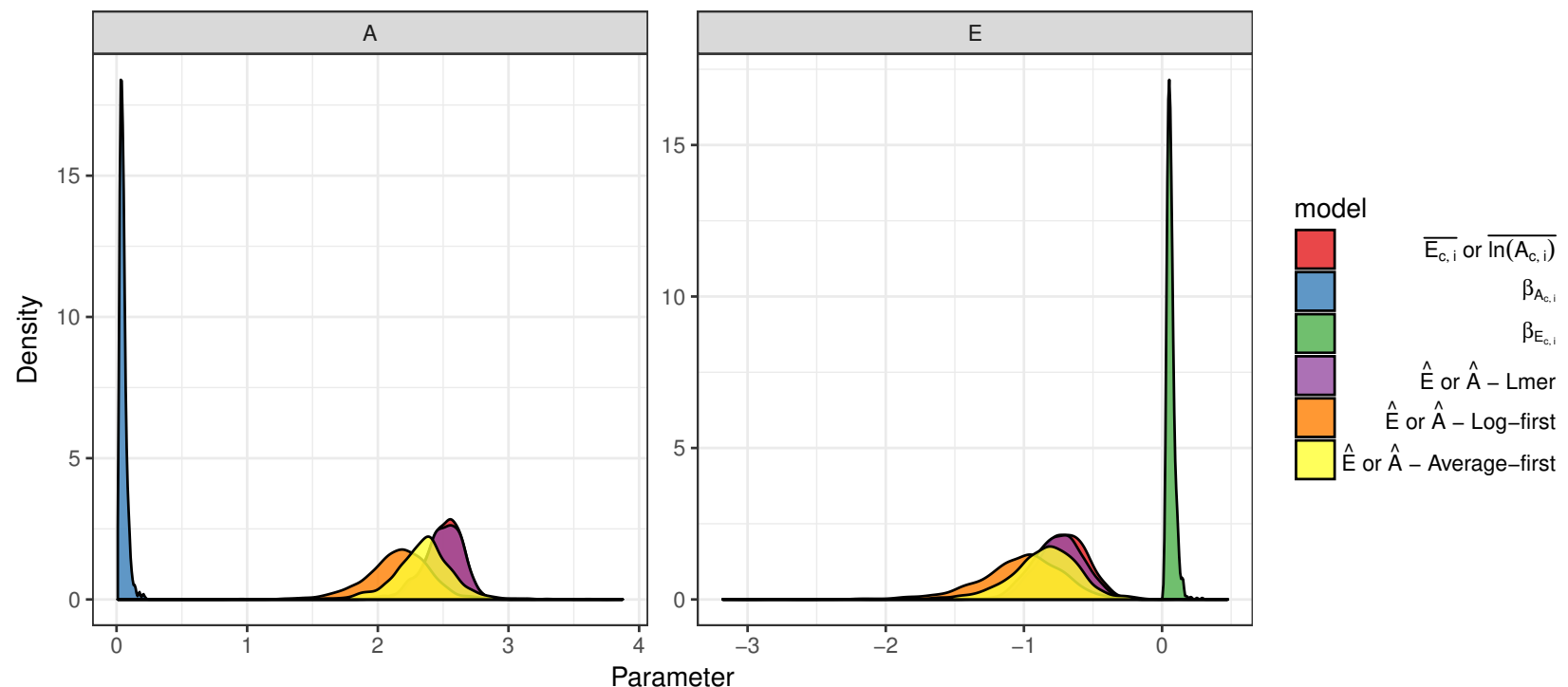

Figure 41: Scenario seven: Comparison between simulated and estimated parameter 


\subsection{Scenario seven: Detailed results on the influnece of chance correlation of}

$\ln A_{c, i} E_{c, i}$ with $\bar{T}_{c}$ on the estimates of $\hat{E}$ and $\hat{A}$

9.4.1 Log-first approach - Best model to explain $\hat{E}$ and $\hat{A}$ based on $\overline{E_{c, i}}, \overline{\ln A_{c, i}}, \beta_{E_{c, i}}$ and $\beta_{A_{c, i}}$
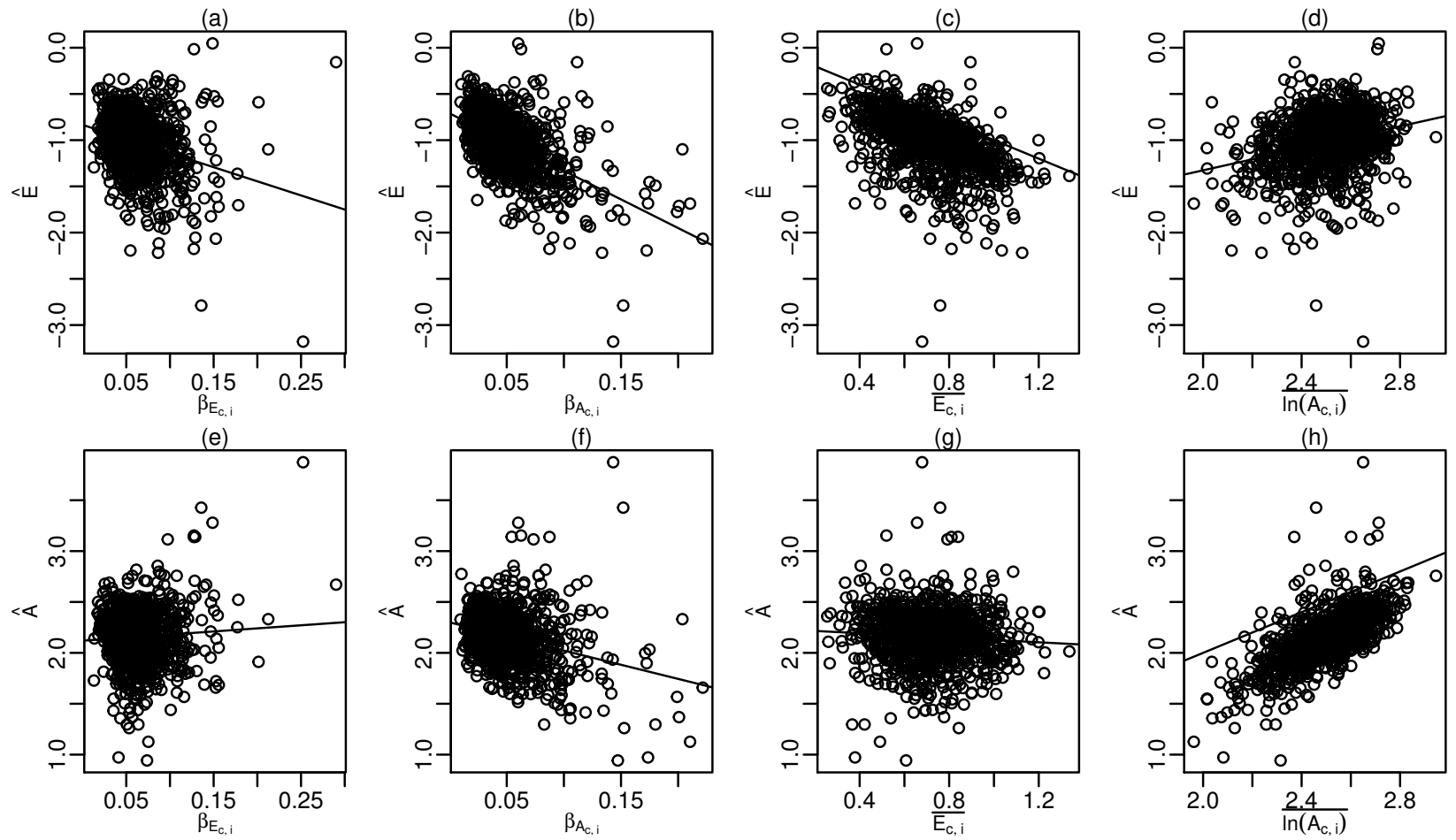

Figure 42: Scenario seven - Log-first approach: Influence of $\beta_{E c, i}, \beta_{A c, i}, \overline{E_{c, i}}$, and $\overline{\ln A_{c, i}}$ on $\hat{E}$ and $\hat{A}$. Solid lines depict linear regression relations for all plot exept (c) and (h) were a bisection line is plotted since identity relations are expected.

Table 74: Scenario seven - Log-first approach: Best model for $\hat{E}$

\begin{tabular}{lrrrr}
\hline & Estimate & Std. Error & t.value & $\operatorname{Pr}(>|t|)$ \\
\hline (Intercept) & -0.706 & 0.283 & -2.496 & 0.013 \\
$\ln A_{c, i}$ & 0.299 & 0.108 & 2.761 & 0.006 \\
$\beta_{A_{c, i}}$ & -8.150 & 0.530 & -15.365 & 0.000 \\
$\beta_{E_{c, i}}$ & 12.958 & 3.789 & 3.420 & 0.001 \\
$\overline{E_{c, i}}$ & -0.987 & 0.040 & -24.895 & 0.000 \\
$\ln A_{c, i}: \beta_{E_{c, i}}$ & -5.170 & 1.471 & -3.515 & 0.000 \\
$\beta_{A_{c, i}}: \beta_{E_{c, i}}$ & 15.101 & 5.604 & 2.694 & 0.007 \\
\hline
\end{tabular}

Table 75: Scenario seven - Log-first approach: Relative importance of best model parameters to explain $\hat{E}$

\begin{tabular}{lr}
\hline & Rel. Importance \\
\hline$\overline{\ln A_{c, i}}$ & 0.062 \\
$\beta_{A_{c, i}}$ & 0.430 \\
$\beta_{E_{c, i}}$ & 0.066
\end{tabular}




\begin{tabular}{lr}
\hline & Rel. Importance \\
\hline$\overline{\overline{E_{c, i}}}$ & 0.419 \\
$\overline{\ln A_{c, i}}: \beta_{E_{c, i}}$ & 0.011 \\
$\beta_{A_{c, i}}: \beta_{E_{c, i}}$ & 0.012 \\
\hline
\end{tabular}

Table 76: Scenario seven - Log-first approach: Best model for $\hat{A}$

\begin{tabular}{|c|c|c|c|c|c|}
\hline & Estimate & Std. & Error & t.value & $\operatorname{Pr}(>|t|)$ \\
\hline (Intercept) & 0.521 & & 0.276 & 1.886 & 0.060 \\
\hline$\overline{\ln A_{c, i}}$ & 0.800 & & 0.106 & 7.539 & 0.000 \\
\hline$\beta_{A_{c, i}}$ & -17.013 & & 2.953 & -5.762 & 0.000 \\
\hline$\beta_{E_{c, i}}$ & -6.767 & & 3.695 & -1.831 & 0.067 \\
\hline$\overline{E_{c, i}}$ & -0.427 & & 0.074 & -5.738 & 0.000 \\
\hline$\frac{{ }_{c, i}}{\ln A_{c, i}}: \beta_{A_{c, i}}$ & 4.616 & & 1.257 & 3.671 & 0.000 \\
\hline$\overline{\ln A_{c, i}}: \beta_{E_{c, i}}$ & 2.392 & & 1.435 & 1.667 & 0.096 \\
\hline$\beta_{A_{c, i}}: \beta_{E_{c, i}}$ & 23.623 & & 5.356 & 4.411 & 0.000 \\
\hline$\beta_{A_{c, i}}: E_{c, i}$ & 5.345 & & 1.271 & 4.206 & 0.000 \\
\hline
\end{tabular}

Table 77: Scenario seven - Log-first approach: Relative importance of best model parameters to explain $\hat{A}$

\begin{tabular}{lr}
\hline & Rel. Importance \\
\hline$\overline{\ln A_{c, i}}$ & 0.774 \\
$\beta_{A_{c, i}}$ & 0.102 \\
$\frac{\beta_{E_{c, i}}}{\overline{E_{c, i}}}$ & 0.025 \\
$\overline{\ln A_{c, i}}: \beta_{A_{c, i}}$ & 0.019 \\
$\overline{\ln A_{c, i}}: \beta_{E_{c, i}}$ & 0.024 \\
$\beta_{A_{c, i}}: \beta_{E_{c, i}}$ & 0.003 \\
$\beta_{A_{c, i}}: \overline{E_{c, i}}$ & 0.031 \\
\hline
\end{tabular}


9.4.2 Average-first approach - Best model to explain $\hat{E}$ and $\hat{A}$ based on $\overline{E_{c, i}}, \overline{\ln A_{c, i}}, \beta_{E_{c, i}}$ and $\beta_{A_{c, i}}$
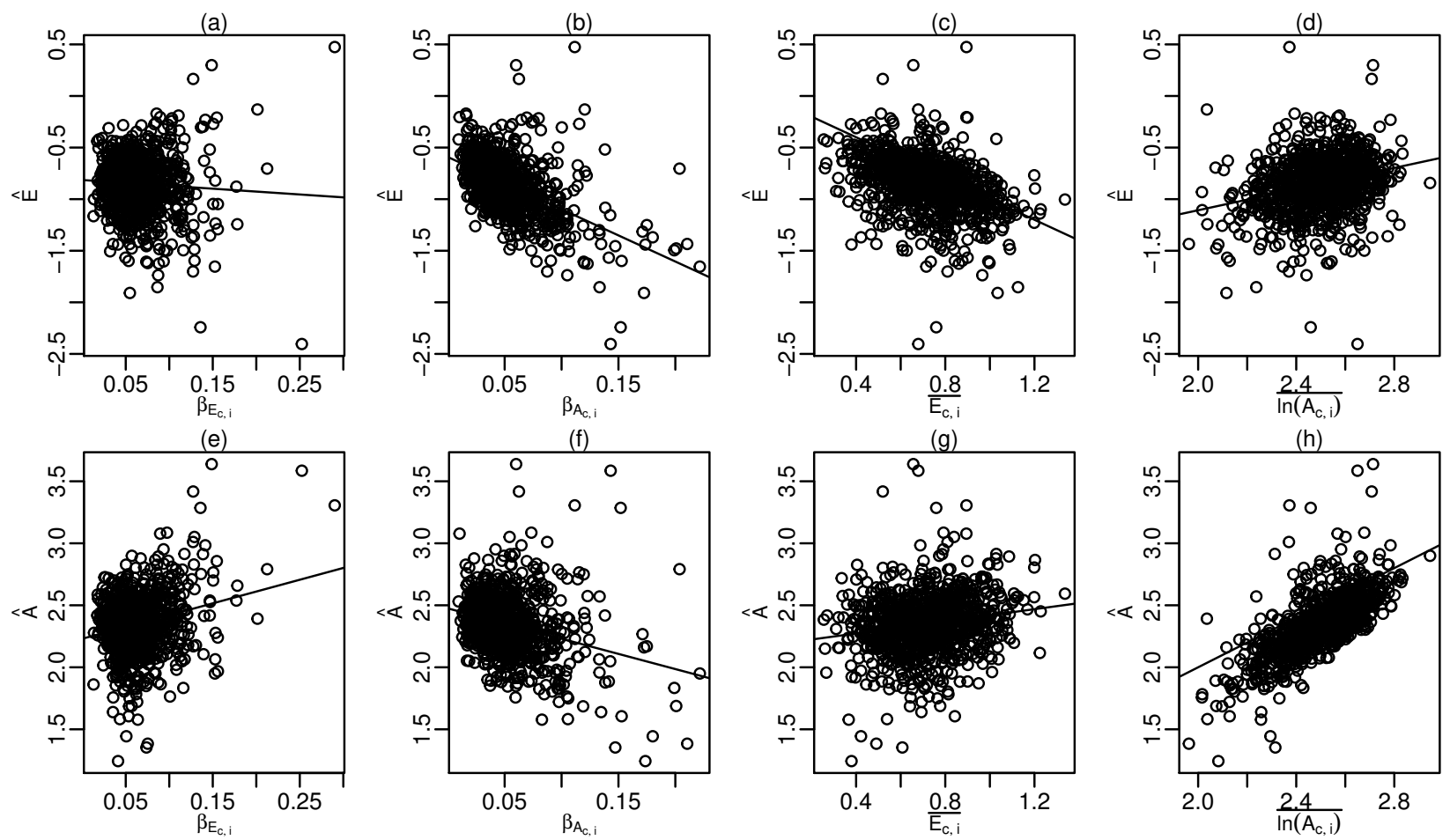

Figure 43: Scenario seven - Average-first approach: Influence of $\beta_{E c, i}, \beta_{A c, i}, \overline{E_{c, i}}$, and $\overline{\ln A_{c, i}}$ on $\hat{E}$ and $\hat{A}$. Solid lines depict linear regression relations for all plot exept (c) and (h) were a bisection line is plotted since identity relations are expected.

Table 78: Scenario seven - average-first approach: Best model for $\hat{E}$

\begin{tabular}{lrrrr}
\hline & Estimate & Std. Error & t.value & $\operatorname{Pr}(>|t|)$ \\
\hline (Intercept) & -0.749 & 0.257 & -2.913 & 0.004 \\
$\frac{\ln A_{c, i}}{\beta_{A_{c, i}}}$ & 0.277 & 0.100 & 2.777 & 0.006 \\
$\beta_{E_{c, i}}$ & -1.756 & 2.750 & -0.638 & 0.523 \\
$\frac{E_{c, i}}{\ln A_{c, i}}: \beta_{A_{c, i}}$ & -0.811 & 0.034 & -23.635 & 0.000 \\
$\ln A_{c, i}: \beta_{E_{c, i}}$ & -3.542 & 1.170 & -2.154 & 0.031 \\
$\beta_{A_{c, i}}: \beta_{E_{c, i}}$ & 14.084 & 1.344 & -2.641 & 0.008 \\
\hline
\end{tabular}

Table 79: Scenario seven - average-first approach: Relative importance of best model parameters to explain $\hat{E}$

\begin{tabular}{lr}
\hline & Rel. Importance \\
\hline$\overline{\ln A_{c, i}}$ & 0.068 \\
$\beta_{A_{c, i}}$ & 0.483 \\
$\beta_{E_{c, i}}$ & 0.046 \\
\hline$E_{c, i}$ & 0.378
\end{tabular}




\begin{tabular}{lr}
\hline & Rel. Importance \\
\hline$\overline{\ln A_{c, i}}: \beta_{A_{c, i}}$ & 0.004 \\
$\overline{\ln A_{c, i}: \beta_{E_{c, i}}}$ & 0.010 \\
$\beta_{A_{c, i}}: \beta_{E_{c, i}}$ & 0.013 \\
\hline
\end{tabular}

Table 80: Scenario seven - average-first approach: Best model for $\hat{A}$

\begin{tabular}{lrrrr}
\hline & Estimate & Std. Error & t.value & $\operatorname{Pr}(>|t|)$ \\
\hline (Intercept) & 0.073 & 0.163 & 0.444 & 0.657 \\
$\overline{\ln A_{c, i}}$ & 0.919 & 0.064 & 14.400 & 0.000 \\
$\beta_{A_{c, i}}$ & -13.071 & 2.286 & -5.718 & 0.000 \\
$\frac{\beta_{E_{c, i}}}{E_{c, i}}$ & 0.357 & 0.349 & 1.024 & 0.306 \\
$\overline{\ln A_{c, i}}: \beta_{A_{c, i}}$ & -0.036 & 0.060 & -0.611 & 0.541 \\
$\beta_{A_{c, i}}: \beta_{E_{c, i}}$ & 3.484 & 0.955 & 3.648 & 0.000 \\
$\beta_{A_{c, i}}: E_{c, i}$ & 19.422 & 4.155 & 4.675 & 0.000 \\
\hline
\end{tabular}

Table 81: Scenario seven - average-first approach: Relative importance of best model parameters to explain $\hat{A}$

\begin{tabular}{lr}
\hline & Rel. Importance \\
\hline$\overline{\ln A_{c, i}}$ & 0.706 \\
$\beta_{A_{c, i}}$ & 0.107 \\
$\beta_{E_{c, i}}$ & 0.100 \\
$\overline{E_{c, i}}$ & 0.033 \\
$\overline{\ln A_{c, i}}: \beta_{A_{c, i}}$ & 0.014 \\
$\beta_{A_{c, i}}: \beta_{E_{c, i}}$ & 0.025 \\
$\beta_{A_{c, i}}: \overline{E_{c, i}}$ & 0.015 \\
\hline
\end{tabular}


9.4.3 LMER approach - Best model to explain $\hat{E}$ and $\hat{A}$ based on $\overline{E_{c, i}}, \overline{\ln A_{c, i}}, \beta_{E_{c, i}}$ and $\beta_{A_{c, i}}$
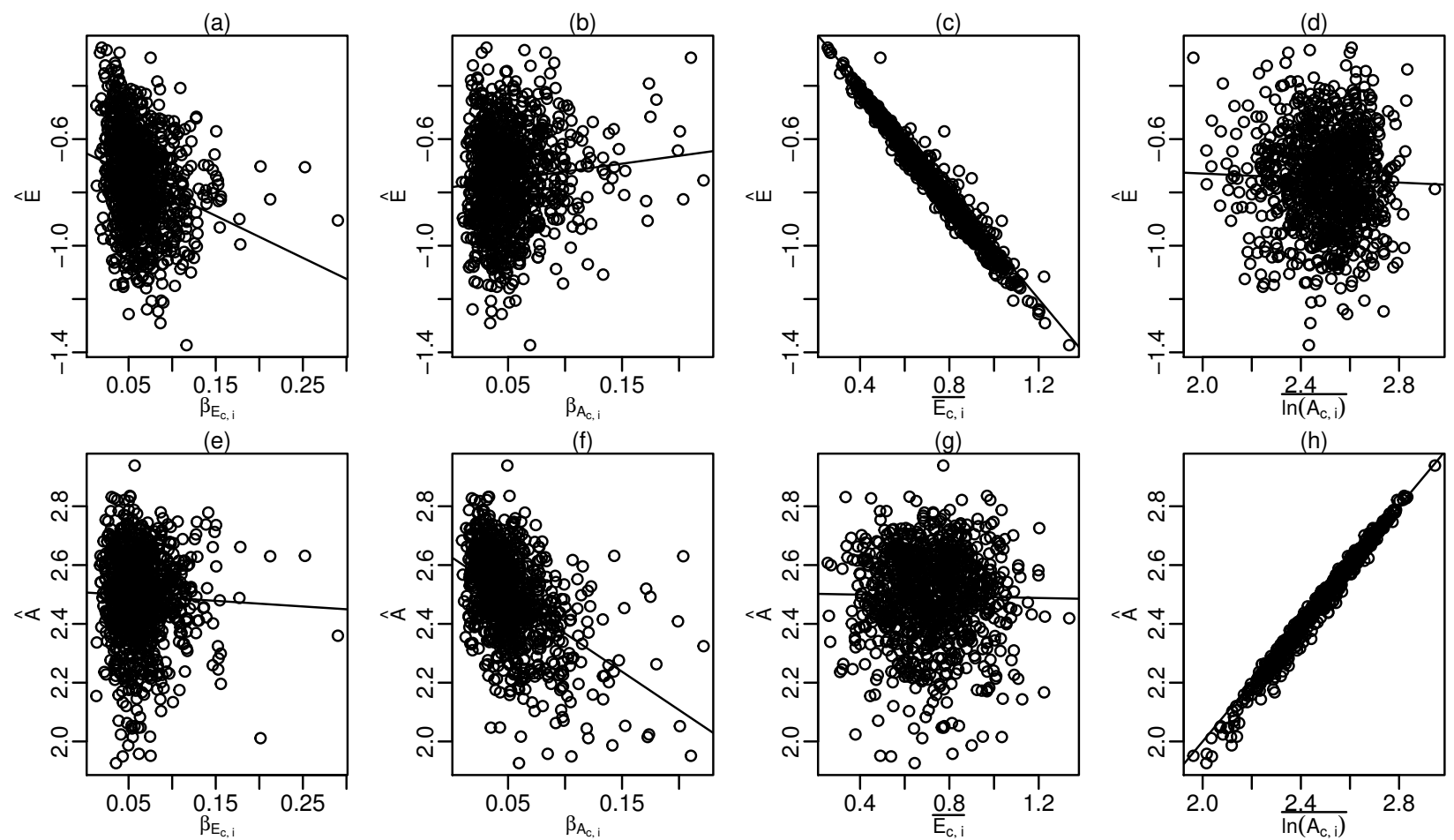

Figure 44: Scenario seven - LMER approach: Influence of $\beta_{E c, i}, \beta_{A c, i}, \overline{E_{c, i}}$, and $\overline{\ln A_{c, i}}$ on $\hat{E}$ and $\hat{A}$. Solid lines depict linear regression relations for all plot exept (c) and (h) were a bisection line is plotted since identity relations are expected.

Table 82: Scenario seven - lmer approach: Best model for $\hat{E}$

\begin{tabular}{lrrrr}
\hline & Estimate & Std. Error & t.value & $\operatorname{Pr}(>|t|)$ \\
\hline (Intercept) & -0.532 & 0.084 & -6.313 & 0.000 \\
$\overline{\ln A_{c, i}}$ & 0.212 & 0.032 & 6.571 & 0.000 \\
$\beta_{A_{c, i}}$ & 4.178 & 0.454 & 9.197 & 0.000 \\
$\frac{\beta_{E_{c, i}}}{E_{c, i}}$ & -1.510 & 0.585 & -2.580 & 0.010 \\
$\overline{\ln A_{c, i}}: \beta_{A_{c, i}}$ & -0.543 & 0.110 & -4.929 & 0.000 \\
$\overline{\ln A_{c, i}}: \beta_{E_{c, i}}$ & -1.679 & 0.187 & -8.989 & 0.000 \\
$\ln A_{c, i}: \bar{E}_{c, i}$ & -0.529 & 0.218 & 2.429 & 0.015 \\
$\beta_{A_{c, i}}: \beta_{E_{c, i}}$ & -1.653 & 0.042 & -5.071 & 0.000 \\
$\beta_{A_{c, i}}: \overline{E_{c, i}}$ & 0.394 & 0.793 & -2.084 & 0.037 \\
$\beta_{E_{c, i}}: \overline{E_{c, i}}$ & 0.507 & 0.226 & 1.741 & 0.082 \\
\hline
\end{tabular}

Table 83: Scenario seven - lmer approach: Relative importance of best model parameters to explain $\hat{E}$

\begin{tabular}{lr}
\hline & Rel. Importance \\
\hline$\overline{\ln A_{c, i}}$ & 0.001 \\
$\beta_{A_{c, i}}$ & 0.011
\end{tabular}




\begin{tabular}{lr}
\hline & Rel. Importance \\
\hline$\beta_{E_{c, i}}$ & 0.038 \\
$\overline{E_{c, i}}$ & 0.947 \\
$\overline{\ln A_{c, i}}: \beta_{A_{c, i}}$ & 0.002 \\
$\overline{\ln A_{c, i}}: \beta_{E_{c, i}}$ & 0.000 \\
$\overline{\ln A_{c, i}}: \overline{E_{c, i}}$ & 0.001 \\
$\beta_{A_{c, i}}: \beta_{E_{c, i}}$ & 0.001 \\
$\beta_{A_{c, i}}: \overline{E_{c, i}}$ & 0.000 \\
$\beta_{E_{c, i}}: \overline{E_{c, i}}$ & 0.000 \\
\hline
\end{tabular}

Table 84: Scenario seven - lmer approach: Best model for $\hat{A}$

\begin{tabular}{lrrrr}
\hline & Estimate & Std. Error & t.value & $\operatorname{Pr}(>|t|)$ \\
\hline (Intercept) & 0.148 & 0.044 & 3.382 & 0.001 \\
$\ln A_{c, i}$ & 0.949 & 0.017 & 55.218 & 0.000 \\
$\beta_{A_{c, i}}$ & -1.167 & 0.260 & -4.487 & 0.000 \\
$\beta_{E_{c, i}}$ & 0.745 & 0.344 & 2.165 & 0.031 \\
$\overline{E_{c, i}}$ & -0.362 & 0.054 & -6.709 & 0.000 \\
$\overline{\ln A_{c, i}}: \beta_{A_{c, i}}$ & 0.336 & 0.110 & 3.045 & 0.002 \\
$\ln A_{c, i}: \beta_{E_{c, i}}$ & -0.309 & 0.129 & -2.395 & 0.017 \\
$\ln A_{c, i}: E_{c, i}$ & 0.143 & 0.021 & 6.665 & 0.000 \\
$\beta_{A_{c, i}}: \beta_{E_{c, i}}$ & 2.575 & 0.465 & 5.537 & 0.000 \\
$\beta_{E_{c, i}}: E_{c, i}$ & -0.283 & 0.114 & -2.487 & 0.013 \\
\hline
\end{tabular}

Table 85: Scenario seven - lmer approach: Relative importance of best model parameters to explain $\hat{A}$

\begin{tabular}{lr}
\hline & Rel. Importance \\
\hline$\overline{\overline{\ln A_{c, i}}}$ & 0.884 \\
$\beta_{A_{c, i}}$ & 0.108 \\
$\frac{\beta_{E_{c, i}}}{E_{c, i}}$ & 0.004 \\
$\overline{\ln A_{c, i}}: \beta_{A_{c, i}}$ & 0.001 \\
$\overline{\ln A_{c, i}}: \beta_{E_{c, i}}$ & 0.000 \\
$\overline{\ln A_{c, i}}: \bar{E}_{c, i}$ & 0.000 \\
$\beta_{A_{c, i}}: \beta_{E_{c, i}}$ & 0.000 \\
$\beta_{E_{c, i}}: \overline{E_{c, i}}$ & 0.002 \\
\hline
\end{tabular}

